\title{
中国植物新分类群、新名称2020年度报告
}

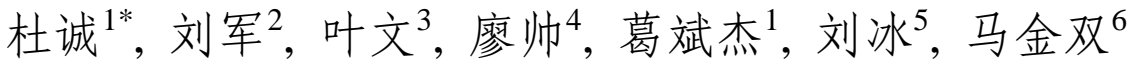

1. 上海辰山植物园(中国科学院上海辰山植物科学研究中心), 上海 201602; 2. 浙江大学图书馆, 杭州 310027; 3. 中国科学院华南植物园, 广州 510650; 4. 华东师范大学生命科学学院, 上海 200241; 5. 中国科学院植物研究所系统与进化植物学国家重点实验室, 北京 100093 ; 6. 北京市植物园, 北京 100093

摘要: 2020年中国共发表高等植物新分类群359个, 其中新属21个、新种312个、新亚种6个、新变种14个、新变型6个; 发表 新组合(等级) 217 个, 新名称 11 个; 发表国家级新记录 48 个; 将 85 个名称处理为 65 个名称的异名; 对 14 个名称进行了应用订 正; 新指定后选(新)模式物种 147 个; 还新发现多年未曾发现的物种 2 个, 排除物种分布 3 个。新发表的物种中, 苔类植物门有 3 个、藓类植物门 3 个、䁬类植物门 6 个、裸子植物门1 个新杂交种、被子植物门 299 个。这些新物种中, 有 86 个发表的同时提供 了详细的分子证据, 78 个在发表时就依据IUCN标准被评估处于受威胁的状态。云南、西藏、广西和四川等西南4省区发表新 物种最多, 占全国新物种发表总数的 $2 / 3$; 新物种发现密度最高的省级行政单位是云南、海南、台湾、广西和浙江等省区; 墨 脱县是新物种发现数量最多的县级行政单位。2020年中国高等植物净新增 294 个分类群, 占全国总数的 $0.79 \%, 313$ 个高等植物 名称发生变动, 占全国总数的 $0.84 \%$ 。

关键词: 高等植物; 新分类群; 名称变动; 中国

杜诚, 刘军, 叶文, 廖帅, 葛斌杰, 刘冰, 马金双 (2021) 中国植物新分类群、新名称 2020年度报告. 生物多样性, 29, 1011-1020. doi: 10.17520/biods.2021122.

Du C, Liu J, Ye W, Liao S, Ge BJ, Liu B, Ma JS (2021) Annual report of new taxa and new names for Chinese plants in 2020. Biodiversity Science, 29, 1011-1020. doi: 10.17520/biods.2021122.

\section{Annual report of new taxa and new names for Chinese plants in 2020}

Cheng $\mathrm{Du}^{1 *}$, Jun $\mathrm{Liu}^{2}$, Wen $\mathrm{Ye}^{3}$, Shuai Liao ${ }^{4}$, Binjie $\mathrm{Ge}^{1}$, Bing Liu ${ }^{5}$, Jinshuang $\mathrm{Ma}^{6}$

1. Shanghai Chenshan Botanical Garden, Shanghai Chenshan Plant Science Research Center, Chinese Academy of Sciences, Shanghai 201602

2. Zhejiang University Library, Hangzhou 310027

3. South China Botanical Garden, Chinese Academy of Sciences, Guangzhou 510650

4. School of Life Sciences, East China Normal University, Shanghai 200241

5. State Key Laboratory of Systematic and Evolutionary Botany, Institute of Botany, Chinese Academy of Sciences, Beijing 100093

6. Beijing Botanical Garden, Beijing 100093

\section{ABSTRACT}

Aim: China has the richest and most diverse flora in the northern hemisphere, with more than 37,098 accepted species of higher plants throughout its vast territory. Each year more than 200 new species from China have been described, but these data have not been updated in international databases expediently. To meet this need, it is necessary to produce an annual report on new taxa, name changes, and latest statuses of higher plants in China.

Methods: We collected information about new taxa and name changes for Chinese higher plants by searching 231 journals and books and 405 articles published in 66 journals and 2 books. We compiled the information and checked the scientific name, author, location, type specimen, and additional relevant information for each species.

Results: In 2020, a total of 359 new taxa of higher plants described from China were reported, including 21 new genera, 312 new species, 6 new subspecies, 14 new varieties, and 6 new forms, as well as 217 new combinations and names at new ranks and 11 new replacement names. In addition, 48 plants were new records for China, 85 names were determined as synonyms of 65 taxa, 14 names were revised, lectotypes or neotypes were designated for 147 names, 2

收稿日期: 2021-04-01; 接受日期: 2021-05-11

基金项目: 上海市绿化和市容管理局科学技术项目(G182416)和中国国家标本资源平台2018年度专项

* 通讯作者 Author for correspondence. E-mail: caragana_tu@hotmail.com 
species were rediscovered, and 3 species were excluded from China. These newly described species belong to Marchantiophyta (3 species), Bryophyta (3 species), Pteridophyta (6 species), Gymnospermae (1 new nothospecies), and Magnoliophyta (299 species). A total of 86 new species were published with detailed molecular evidence, and 78 new species were evaluated as threatened species according to IUCN standards. Four southwestern provinces, including Yunnan, Xizang, Guangxi, and Sichuan, were the most important sources of new discoveries, accounting for two thirds of the total number of new species published in China. Yunnan, Hainan, Taiwan, Guangxi, and Zhejiang had the highest density of newly discovered species.

Conclusions: In 2020 in China, a net of 294 (0.79\% of the total number) new plant taxa were recorded and 313 (0.84\% of the total number) plant names were changed. 2020 was undoubtedly a fruitful year in plant taxonomic research, with the largest increase in the number of species discovered, the number of taxonomic revisions made, and the number of articles published on Chinese higher plants since 2000.

Key words: higher plants; new taxa; new name changes; China

据最新报道, 中国有37,098种高等植物(The Biodiversity Committee of Chinese Academy of Sciences, 2020), 在北温带, 中国无疑拥有最丰富、 最多样化的植物区系(Hong \& Blackmore, 2015)。丰 富的生物多样性和稳定的专业分类学研究人员的 高度匹配, 使中国从20世纪90年代开始就一直位于 国家年度维管植物新物种发现和命名的前三名。英 国皇家植物园 (邱园)根据国际植物名称索引 (International Plant Names Index, IPNI, http://www.ip ni.org/)的统计, 平均每年有超过 100 个维管植物新 物种在中国被描述发表(Willis \& Bachman, 2016; Willis, 2017; Antonelli et al, 2020)。由于国际数据库 对包括中国在内的区域性植物新物种发表资料存 在一定程度的遗漏, 2019年中国维管植物新物种发 表数量实际超越了位于赤道并拥有世界最大面积 热带雨林的巴西, 成为当年全世界发现植物新物种 最多的国家(Antonelli et al, 2020; Du et al, 2020)。详 细的统计数据显示, 中国平均每年发表 220 个维管 植物新分类群, 发现 32 个国家级新记录, 世界上每 年新发表的维管植物类群有 $1 / 10$ 来自中国, 且没有 明显减缓的趋势(Du et al, 2020)。可见被世界公认为 北温带植物大国的中国仍有大量植物等待被发现。

尽管大多数植物新物种都发表在国际性刊物 或主要的国内植物学专业期刊上, 但仍然有部分物 种不能及时被国际数据库收录, 特别是发表在综合 性刊物、地方性刊物或一些书籍中的分类群。对这 些分散于各种书籍及刊物中的有关于植物新分类 群、名称变化及新发现等植物新资料, 有必要进行 年度性的汇总, 从而方便学者发现和引用, 并对处 理不当的类群及时进行分类学处理。

我们系统检索和整理了曾经发表植物类群或
名称的210种期刊, 并通过中国知网(https://www.cn ki.net/)、IPNI和Tropicos (http://www.tropicos.org/)检 索到21个新增期刊和 2 部书籍中也有植物名称的发 表。通过系统梳理发现：2020年度有66个期刊发表 的 405 篇文章内容涉及到高等植物的新分类群(新 属、新杂交属、新种、新杂交种、新亚种、新变种、 新变型)、新组合(等级)名称、新名称、国家级新记 录、新异名、后选(新)模式指定以及名称应用订正 等信息。这些新分类群和名称变化将会对中国的植 物物种编目带来影响, 特别是植物志、植物名录等 涉及植物名称的著作编写。

经过数据清理和统计, 2020年共发表中国境内 高等植物新分类群359个, 其中新属21个(含1个杂 交属); 新种 312 个(含 3 个杂交种), 新种下等级 26 个 (其中亚种6个、变种14个、变型6个); 新组合(等级) 217 个, 新名称 11 个。发表国家级新记录 48 个; 将 85 个名称处理为 65 个名称的异名; 新指定后选(新)模 式物种 147 个; 还对 14 个名称进行了应用订正; 新 发现多年未曾发现的物种 2 个, 排除了 3 个物种在中 国的分布。

我们整理形成了中国植物新分类群文献目录 2020 (附录1), 将2020年发表的高等植物新分类群、 新记录类群、名称变动、模式化及其他涉及名称变 动的情况以条目的形式给出。该目录按照科名字母 顺序排列, 每个科内按照字母顺序排列条目。每个 条目包含了物种学名、具体分类学处理、发表文献 信息和分布信息, 以及文章对应的doi编号。对于新 物种还给出了 IUCN评估等级和物种发表证据等 级。列出了本年度所有收录期刊的详细信息, 并展 示了2020年新发表高等植物物种的模式标本及其 采集地、采集时间、标本馆藏地等信息，方便查阅。 


\section{1 新发表物种的基本状况}

2020年是1990年以来中国高等植物新种发表 数量最多的一年, 达到312个。其原因与分类学文章 发表数量显著提高(平均为 200 篇/年)和类群专著 (Harber, 2020)中大量物种集中发表有直接关系。

2020 年发表的 312 个新种中, 苔类植物门有 3 个、藓类植物门 3 个、蕨类植物门 6 个、被子植物门 299 个, 以及裸子植物门 1 个新杂交种。苔藓植物、 偋类植物和裸子植物的新物种发表数量显著少于 被子植物。312个新种中有86个在发表文献中提供 了详细的分子系统学研究证据, 占新物种总数的 27.6\%。这些包含分子系统学证据的新种全部发表 在期刊中, 特别是被SCI收录的期刊, 发表在专著 中的新种则基本没有包含或引用分子证据。在发表 新分类群时, 叶绿体基因组的 $r b c \mathrm{~L} 、 m a t \mathrm{~K}$ 、 $t r n H-p s b \mathrm{~A}$ 、trnL-F和核基因组的ITS片段最常作为 构建系统发育树的序列。尽管还有近3/4的物种依据 形态学(也包括种皮、孢粉、叶表皮等微形态特征) 证据发表, 但分子系统学证据已逐渐成为国际期刊 审稿人关注的重要证据之一。

312 个新种有 115 个依据IUCN标准评估了濒危 等级, 其中 39 个被评为极危(CR)、17个为濒危(EN)、 18 个为易危 $(\mathrm{VU}) 、 4$ 个为近危 $(\mathrm{NT}) 、 10$ 个为无危 (LC)、 27 个数据缺乏(DD); 剩余 197 个物种没有评估, 但有 4 个被认为属于濒危, 但没有依据IUCN标准给 出依据。可见新发表的物种中超过 $1 / 4$ 在发表时已经 处于受威胁状态。

312 个新种中有 235 个是基于作者团队自己采 集到的标本描述发表, 占总数的 $75.3 \%$, 其他 77 个 是依据其他团队采集的馆藏标本描述发表。这些新 物种中有14种在采集的当年被发表, 共170种在采 集后3年内发表, 196种在采集后5年内发表, 243种 在采集后10年内发表, 289种在采集后30年内发表, 仅有 5 个新种的模式标本采自于 100 年前(最早的标 本采于1904年)。出现次数最多的采集队是Boufford 主导的中美联合植物考察队, 其他如青藏高原采集 队、高黎贡山采集队等均只有零星出现。

一个物种从标本采集到最终被描述发表的时 间被称为物种的“橱柜时间”。所有生物类群的平均 梪柜时间为21年，植物的平均梪柜时间则超过了 30 年(Fontaine et al, 2012)。1970-2010年间，仅有16\%
的植物物种在被采集到的 5 年内得到初步描述发表, 大约 $1 / 4$ 植物物种的梪柜时间则超过了 50 年(Bebber et al, 2010)。我们的统计数据显示, 2020年新发表中 国植物物种的梪柜时间显著缩短至10.8年, $62.8 \%$ 的 植物物种在被采集到的5年内得到描述发表, $77 \%$ 的 物种在被采集到的10年内得到描述发表。这些数据 表明, 近些年来中国植物分类学工作者的野外采集 活动比较活跃, 采集到的标本也能够及时处理。此 外采集新鲜材料可提供分子及细胞学研究为物种 的发表提供更客观的证据，也是将模式指定为新近 采集标本的原因之一。仅有不到 $8 \%$ 的物种是根据或 引证采集30年以上的标本发表, 也暴露出学者们对 标本馆丰富历史标本研究的缺位。

\section{新发表的属级分类群}

2020年中国高等植物共发表了 22 个新属名, 代 表了 21 个新的属级分类群, 以及 1 个属因重名而给 出的新名称。这些新属都属于维管植物, 其中硕类 植物门3个, 被子植物门18个。除了 Nesom (2020a, b, c, d, e, f, g)发表的10个自菊科紫苑属(Aster L.)独立 出的小属(表1)外, 余下的11属均有详细的分子系统 学及形态学证据, 并且都是自传统的形态学属中独 立出来的单系类群。这11个单系新属包括:

(1)菊科: 广义鸦葱属(Scorzonera L. S. 1.) 独立 出拐轴鸦葱属 (Lipschitzia Zaika, Sukhor. \& N. Kilian), 并恢复毛鸦芴属(Gelasia Cass.)、鸦憼属 (Takhtajaniantha Nazarova) 、假柄果菊属 (Pseudopodospermum Kuth.)等几个单系小属的属级 地位(Zaika et al, 2020); 曾经被分散置于莴苣属 (Lactuca L.)和耳菊属(Nabalus Cass.)的单系群合并 组建中国苣属(Sinoseris N. Kilian, Ze H. Wang \& H. Peng) (Wang ZH et al, 2020); 夢吾属(Ligularia Cass.) 线苞组(Sect. Stenostegia Pojark.)提升为无鞘夢吾属 (Vickifunkia C. Ren, Long Wang, I. D. Illar. \& Q. E. Yang) (Ren et al, 2020)。

(2)兰科: 并系类群羊耳蒜属 (Liparis Rich.)㫸唇 兰组(Sect. Blepharoglossum Schltr.)提升为睫唇兰属 (Blepharoglossum (Schltr.) L. Li) (Li L et al, 2020); 拟万代兰属 (Vandopsis Pfitz.) 独立出船唇兰属 (Cymbilabia D. K. Liu \& Ming H. Li) (Liu et al, 2020)。

(3)水龙骨科: 瓦韦属(Lepisorus (J. Sm.) Ching) 
表1 G. L. Nesom 2020年发表的新属中涉及中国类群的统计

Table 1 New genus based on Chinese taxa published by G. L. Nesom in 2020

\begin{tabular}{llll}
\hline $\begin{array}{l}\text { 中文属名 } \\
\text { Chinese name of the genus }\end{array}$ & $\begin{array}{l}\text { 属学名 } \\
\text { Scientific name of the genus }\end{array}$ & $\begin{array}{l}\text { 模式种基名 } \\
\text { Basionym of type species }\end{array}$ & $\begin{array}{l}\text { 包含物种数量 } \\
\text { Number of species }\end{array}$ \\
\hline 东风菜属 & Cardiagyris G. L. Nesom & Aster scaber Thunb. & 4 \\
镰叶紫苑属 & Chaochienchangia G. L. Nesom & Aster falcifolius Hand.-Mazz. & 1 \\
长柄马兰属 & Cordiofontis G. L. Nesom & Aster peduncularis Wall. ex Nees & 1 \\
巴塘紫苑属 & Geothamnus G. L. Nesom & Aster batangensis Bureau \& Franch. & 1 \\
褐毛紫苑属 & Griersonia G. L. Nesom & Aster fuscescens Bureau \& Franch. & 3 \\
重羽紫菀属 & Iteroloba G. L. Nesom & Aster bipinnatisectus Ludlow ex Grierson & 1 \\
灰枝紫苑属 & Sinobouffordia G. L. Nesom & Aster poliothamnus Diels & 2 \\
小舌紫苑属 & Sinosidus G. L. Nesom & Amphirhapis albescens DC. & 8 (9变种 9 varieties) \\
重冠紫苑属 & Tibetiodes G. L. Nesom & Heterochaeta diplostephioides DC. & 25 \\
横斜紫茤属 & Yonglingia G. L. Nesom & Aster hersileoides C. K. Schneid. & 2 \\
\hline
\end{tabular}

独立出印度瓦韦属(Ellipinema Li Bing Zhang \& Liang Zhang) (Zhang L et al, 2020a); 宽带蕨属 (Platygyria Ching \& S. K. Wu) 耳基宽带蕨 (Platygyria $\times$ inaequibasis Ching \& S. K. Wu) 被确认 为印度瓦韦属和瓦韦属属间杂交新属耳基宽带䓲属 (×Ellipisorus Li Bing Zhang \& Liang Zhang; $\times$ Lepinema Li Bing Zhang \& Liang Zhang nom. illeg.) (Zhang \& Zhang, 2020a, b)。

(4)木槉科: 木樨属(Osmanthus Lour.)圆雉花序 组(Sect. Leiolea (Spach) P. S. Green)被提升为万钧 木属(Chengiodendron C. B. Shang, X. R. Wang, Yi F. Duan \& Yong F. Li) (Li YF et al, 2020)。

(5)瓶尔小草科: 小阴地蕨属 (Botrychium Sw.) 中独立出单系分支劲直阴地濒属(Sahashia Li Bing Zhang \& Liang Zhang) (Zhang L et al, 2020b)。

(6)禾本科: 竹亚科箬竹属(Indocalamus Nakai) 中新成立单型属雷文竹属(Ravenochloa D. Z. Li \& Y. X. Zhang) (Zhang YX et al, 2020); 赤竹属(Sasa Makino \& Shibata)中国类群新成立单系类群华赤竹 属(Sinosasa L. C. Chia ex N. H. Xia, Q. M. Qin \& Y. H. Tong) (Qin et al, 2020)。

\section{3 新分类群所属的科属统计}

2020年发表的338个种及种下新分类群隶属于 78科161属。除Harber (2020)在小檗科专著中集中发 表的63个新种外, 其他类群仍然集中在传统的兰 科、菊科、天门冬科、毛茛科、禾本科、蓄薇科、 伞形科、豆科、唇形科等物种较多的大科。中国西 南地区的热带、亚热带石灰岩地区多样性水平较高 的苦苣苔科、凤仙花科、秋海棠科、报春花科、马
兒铃科也有较多的新分类群。

小檗科发表的 63 个新种全部属于小檗属 (Berberis L.)。分布于甘肃(南部)、青海、四川、西 藏、云南等青藏高原及横断山地区。

苦苍苔科共发表 42 个新种和 1 个新变种, 最多的 2 个属是马铃苣苔属(Oreocharis Benth.)和报春菅苔 属(Primulina Hance), 各10个新种。主要分布在广西、 贵州、西藏、云南等中国西南热带、亚热带地区。

兰科共发表 20 个新种、 1 个新杂交种、 6 个新变 种、 1 个新变型, 最多的 2 个属是石豆兰属 (Bulbophyllum Thouars) (3新种、1新变种)和兰属 (Cymbidium Sw.) (3新种、1新杂交种)。主要分布在 台湾、西藏、云南等中国南方地区。

菊科共发表20个新种, 最多的3个属是风毛菊 属(Saussurea DC.) (5种)、紫苑属 (Aster L.) (3种)和蒲 公英属(Taraxacum F. H. Wigg.) (3种)。主要分 布在新 疆、西藏等高海拔地区和中国南方地区。

凤仙花科共发表13个新种, 全部属于凤仙花属 (Impatiens L.)。主要分布在广西、西藏等中国西南 热带、亚热带地区。

秋海棠科共发表12个新种, 全部属于秋海棠属 (Begonia L.)。主要分布在广西、云南等中国西南热 带、亚热带地区。

发表 5 个及以上新分类群的科还有天门冬科(8 种)、毛茛科(8种)、禾本科(7新种、1 1 新变种)、蓄薇 科(4新种、1新亚种、1 1 新变型)、伞形科(5种)、豆科 (5种)、唇形科(5种)、报春花科(5种)和马兒铃科(5种)。

\section{新分类群和新记录物种的省级分布}

2020年发表的中国高等植物新物种、国家级新 
记录物种以及新种下等级类群主要分布在云南、西 藏、广西、四川等西南省区。云南省内发表了97个 新物种、 5 个种下新分类群 (1个新亚种和 4 个新变种) 以及 11 个国家级新记录物种, 大约占到了当年中国 植物新物种数量的 $1 / 3$ 。此外西藏(36个新种、 1 个新 亚种、1 1 个新变种和 14 个新记录)、广西(38 个新种、 1 个新亚种、 2 个新变种和 10 个新记录)、四川(42个 新种、 1 个新记录)等也发现了大量的新物种和新记 录物种。总面积仅占中国陆地总面积 $1 / 4$ 的 4 个省区 新物种发表数量占到了当年中国植物新物种数量 的 $2 / 3$ 。总体上, 物种发表数量从西南到东北呈现减 少趋势(图1)。

排除面积因素，新物种发现数量最多的 5 个省 级单位分别是云南、海南(8个新种、1 1 个新变种和 3 个新记录)、广西、浙江(17个新种、3个新亚种、 4 个新变种、 2 个新变型和 3 个新记录)和台湾(7个新 种、 1 个新变种、 2 个新变型和 6 个新记录)。云南省 属于热带及亚热带山地, 地形复杂, 被国内外学者 广泛重视, 是单位面积发现物种最多的省份。海南 省和台湾省因属于南部岛屿并具有丰富的生境以 及与大陆隔离, 也有很高的物种发现密度。浙江省 位于中国东部沿海, 物种多样性水平理论上应与邻 近的自然条件基本一致的安徽( 2 个新种、 1 个新变 种、 1 新变型和 1 个新记录)、福建( 5 个新种和 1 个新
变种)和江西(4个新种)基本相当, 但物种发现密度 高于邻近省区。这种情况可能与该省植物学者全力 推动《浙江植物志》再版工作有很大关系: 因为该 植物志不在正文或附录中处理类群, 所有的分类学 处理均另文在期刊中提前发表(丁炳扬, 2020)。

发表新物种最多的县级行政单位是西藏自治 区的墨脱县, 2020年有超过19个高等植物新种被发 表, 但其中16个是由国外学者研究并发表在国外期 刊上。特别是在被非法占据的中国领土上，丰富的 生物多样性无法被中国学者及时研究, 令人非常遗 憾。此外云南省西北部的香格里拉(10个)、贡山(5 个), 东南部的麻栗坡( 9 个)、文山(6个)、河口(4个), 西部的盈江(5个), 四川省西部的康定(6个)、木里(4 个), 广西壮族自治区西南部的靖西(5个), 海南省 东南部的陵水(4个)和浙江省西南部的景宁(4个)都 有较多的高等植物新种发表。

北京、天津、上海、宁夏、河北、黑龙江、吉 林、山西和澳门没有任何有关于新物种、国家级新 记录物种的报道。这些省、区、市大都位于中东部 地区，植物多样性调查已经基本完成。

\section{发表植物新分类群的学者}

2020 年发表的 338 个种及种下新分类群由 238 个中外作者团队发表。其中 210 个中国作者团队发

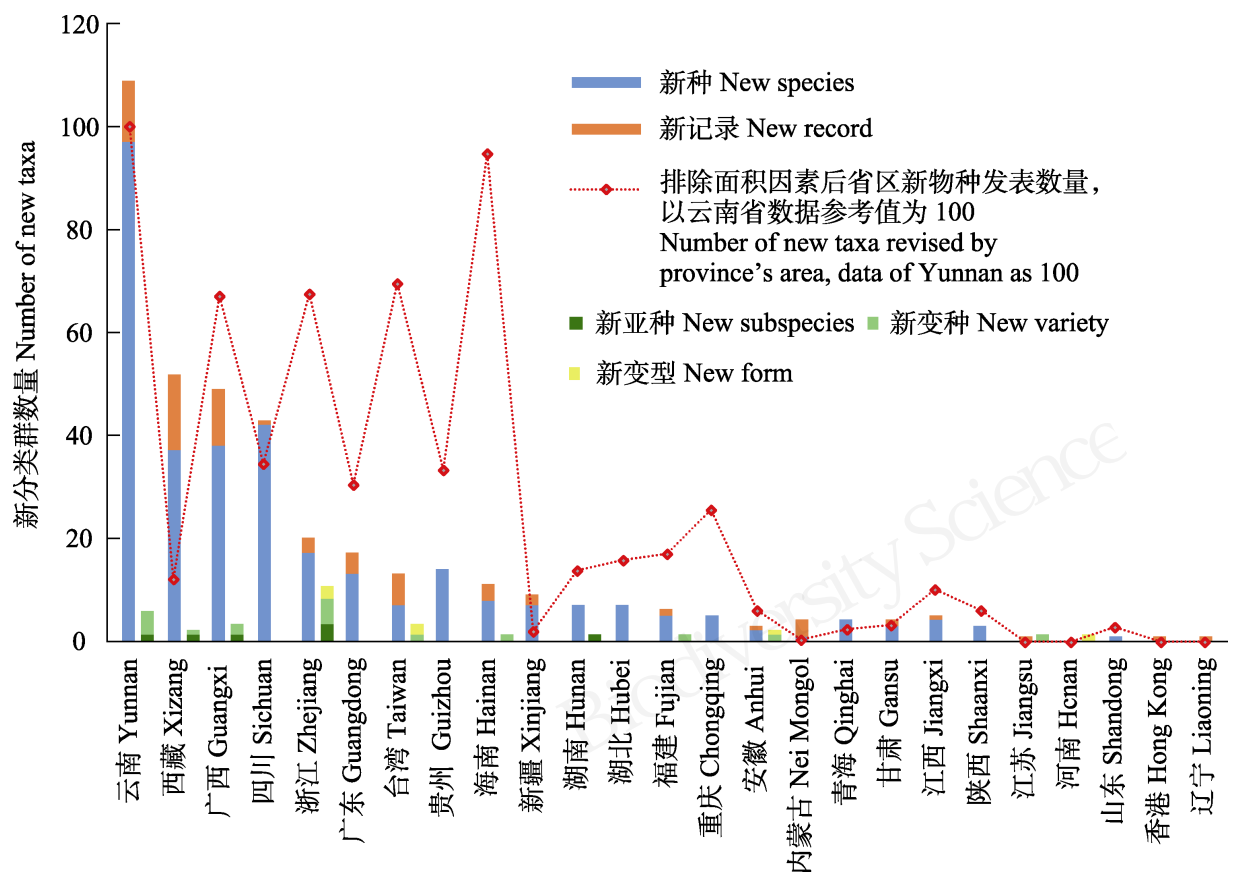

图12020年中国各省区发表的新分类群和国家级新记录物种数量

Fig. 1 The provincial number of Chinese new taxa and new recorde species published in 2020 
表了 243 个种及种下新分类群, 23 个国外作者团队 发表了 90 个种及种下新分类群, 5 个中外合作团队 发表了剩下的 5 个新分类群。这些中外作者团队中 有中国学者 298 人, 外国学者44人。2020年发表中国 植物物种数量最多的中国学者是陈征海 $(Z . H$. Chen, 19 个种及种下新分类群), 外国学者是Julian F. Harber (Harber, 63个种及种下新分类群)。发表5个以 上分类群的作者见表2。

2020 年参与高等植物命名的中国学者有 298 人, 其中 100 人是第一次参与命名的学者。这些首次参 与命名的学者大多是年轻的植物分类学者或植物 分类学专业的研究生。2000年以来平均每年有62位 新增学者参与到维管植物的命名工作中来, 2007年 以来每年增加人数都超过了平均值, 2019年甚至达 到了新增117人的历史极值。但这些新增学者中大 多数仅发表过 1 个分类群, 并且之后不再参与植物 命名工作。图2展示了1916年以来每年新参与命名
的中国维管植物命名人数量。

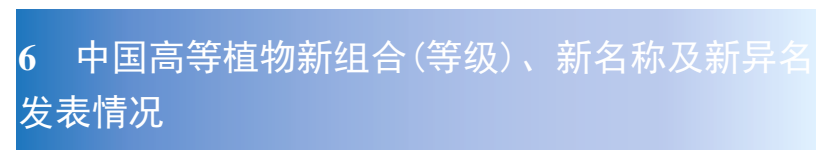

2020年中国高等植物新发表了 217 个新组合(等 级)和 13 个新名称(全部属于维管植物), 有 2 个新名 称是为了建立新属或替代不合法属名而进行的名 称替代, 代表了195个种(185个组合、11个新名称, 其中 1 个物种被组合了 2 次)、1个亚种、25个变种和 6 个变型的名称变化。1 85 个物种的组合和 11 个物种 的新名称有 77 个是因建立新属而进行的名称组合。

新组合(等级)名称及新名称带来的名称变化主 要集中在菊科(77个)、木兰科(33个)、五加科(19个)、 蓄薇科(19个)、兰科(13个)、豆科(11个)等几个较受 关注的科。前 6 个科共有 172 个名称组合, 约占当年度 名称组合总量的 $3 / 4$, 剩余 29 个科发表了剩余 55 个名 称组合。被关注的类群集中程度较新物种发表更大。

\section{表2 2020 年发表 5 个及以上新分类群的中外学者统计}

Table 2 Statistics of Chinese and foreign authors who published five new taxa or more in 2020

\begin{tabular}{|c|c|c|c|c|c|}
\hline $\begin{array}{l}\text { 标准拼写 } \\
\text { Stand name }\end{array}$ & $\begin{array}{l}\text { 姓名 } \\
\text { Name }\end{array}$ & $\begin{array}{l}\text { 西文拼写 } \\
\text { Roma name }\end{array}$ & $\begin{array}{l}\text { 研究领域 } \\
\text { Research area }\end{array}$ & $\begin{array}{l}\text { 研究地区 } \\
\text { Research region }\end{array}$ & $\begin{array}{l}\text { 新分类群数 } \\
\text { No. of new taxa }\end{array}$ \\
\hline Z. H. Chen & 陈征海 & Chen, Zheng Hai & 浙江植物 Plants of Zhejiang & 浙江 Zhejiang & 19 \\
\hline Yan Liu & 刘演 & Liu, Yan & 广西植物 Plants of Guangxi & 广西 Guangxi & 16 \\
\hline F. Wen & 温放 & Wen, Fang & 苦菅苔科和莳麻科 Gesneriaceae and Urticaceae & 中国 China & 13 \\
\hline Lei Cai & 蔡否 & Cai, Lei & 天门冬科和苦芭苔科 Asparagaceae and Gesneriaceae & 中国 China & 10 \\
\hline W. H. Chen & 陈文红 & Chen, Wen Hong & 云南植物 Plants of Yunnan & 云南 Yunnan & 10 \\
\hline Y. M. Shui & 税玉民 & Shui, Yu Min & 云南植物 Plants of Yunnan & 云南 Yunnan & 10 \\
\hline X. F. Jin & 金孝锋 & Jin, Xiao Feng & 浙江植物 Plants of Zhejiang & 浙江 Zhejiang & 8 \\
\hline G. Y. Li & 李根有 & Li, Gen You & 浙江植物 Plants of Zhejiang & 浙江 Zhejiang & 7 \\
\hline W. T. Wang & 王文采 & Wang, Wen Tsai & 毛茛科 Ranunculaceae & 中国 China & 7 \\
\hline C. I Peng & 彭镜毅 & Peng, Ching I & 台湾植物 Plants of Taiwan & 台湾 Taiwan & 6 \\
\hline W. Y. Xie & 谢文远 & Xie, Wen Yuan & 浙江植物 Plants of Zhejiang & 浙江 Zhejiang & 6 \\
\hline Z. B. Xin & 辛子兵 & Xin, Zi Bing & 苦芭苔科 Gesneriaceae & 广西 Guangxi & 6 \\
\hline B. Pan & 盘波 & Pan, Bo & 天门冬科和苦苣苔科 Asparagaceae and Gesneriaceae & 广西 Guangxi & 5 \\
\hline D. G. Zhang & 张代贵 & Zhang, Dai Gui & 湖南植物 Plants of Hunan & 中国 China & 5 \\
\hline P. L. Chiu & 装宝林 & Chiu, Pao Ling & 浙江植物 Plants of Zhejiang & 浙江 Zhejiang & 5 \\
\hline Y. F. Lu & 鲁益飞 & Lu, Yi Fei & 浙江植物 Plants of Zhejiang & 浙江 Zhejiang & 5 \\
\hline Z. L. Dao & 刀志灵 & Dao, Zhi Ling & 天门冬科和苦菅苔科 Asparagaceae and Gesneriaceae & 四川和云南 Sichuan and Yunnan & 5 \\
\hline N. H. Xia & 夏念和 & Xia, Nian He & 杜鹃花科和禾本科 Ericaceae and Poaceae & 中国 China & 5 \\
\hline Harber & & Harber, Julian F. & 小檗科 Berberidaceae & 中国和越南 China and Vietnam & 63 \\
\hline D. Borah & & Borah, Dipankar & 中国和印度植物 Plants of India and China & 中国和印度 China and India & 6 \\
\hline Taram & & Taram, Momang & 中国和印度植物 Plants of India and China & 中国和印度 China and India & 5 \\
\hline
\end{tabular}

†彭镜毅(1950-2018), 中国台湾著名植物分类学家, 2020年发表类群为与其他学者合作发表。

$\dagger$ Ching I Peng (1950-2018) is a well-known plant taxonomist in Taiwan of China. The taxa published by C. I Peng were completed with other collaborators in 2020. 
表3 2020年发表5个及以上新组合的中外学者统计

Table 3 Statistics of Chinese and foreign authors who published five new combinations or more in 2020

\begin{tabular}{|c|c|c|c|c|c|}
\hline $\begin{array}{l}\text { 标准拼写 } \\
\text { Stand name }\end{array}$ & $\begin{array}{l}\text { 姓名 } \\
\text { Name }\end{array}$ & $\begin{array}{l}\text { 西文拼写 } \\
\text { Roma name }\end{array}$ & $\begin{array}{l}\text { 研究领域 } \\
\text { Research area }\end{array}$ & $\begin{array}{l}\text { 研究地区 } \\
\text { Research region }\end{array}$ & $\begin{array}{l}\text { 发表新组合数量 } \\
\text { New combinations }\end{array}$ \\
\hline B. B. Liu & 刘涁森 & Liu, Bin Bin & 菩薇科 Rosaceae & 全球 Global & 16 \\
\hline Z. H. Chen & 陈征海 & Chen, Zheng Hai & 浙江植物 Plants of Zhejiang & 浙江 Zhejiang & 8 \\
\hline H. Yu & 余鸿 & Yu, Hong & 木兰科和兰科 Magnoliaceae and Orchidaceae & 云南 Yunnan & 6 \\
\hline K. W. Jiang & 蒋凯文 & Jiang, Kai Wen & 豆科 Fabaceae & 中国 China & 6 \\
\hline Sima & 司马永康 & Sima, Yong Kang & 木兰科 Magnoliaceae & 云南 Yunnan & 6 \\
\hline B. Y. Ding & 丁炳扬 & Ding, Bing Yang & 天南星科和千屈菜科 Araceae and Lythraceae & 浙江 Zhejiang & 5 \\
\hline C. Ren & 任琛 & Ren, Chen & 菊科 Asteraceae & 亚洲 Asia & 5 \\
\hline Long Wang & 王龙 & Wang, Long & 菊科 Asteraceae & 亚洲 Asia & 5 \\
\hline Q. E. Yang & 杨亲二 & Yang, Qin Er & 菊科和毛茛科 Asteraceae and Ranunculaceae & 中国 China & 5 \\
\hline C. B. Callaghan & & Callaghan, Chris B. & 木兰科 Magnoliaceae & 亚洲 Asia & 25 \\
\hline Png & & Png, Siak Khoon & 木兰科 Magnoliaceae & 亚洲 Asia & 25 \\
\hline G. M. Plunkett & & Plunkett, Gregory M. & 伞形科和五加科 Apiaceae and Araliaceae & 全球 Global & 17 \\
\hline Lowry & & Lowry, Porter Prescott & 五加科 Araliaceae & 全球 Global & 17 \\
\hline J. Wen & 文军 & Wen, Jun & 蓄微科 Rosaceae & 全球 Global & 16 \\
\hline Li Bing Zhang & 张丽兵 & Zhang, Li Bing & 偋类植物 Pteridophytes & 全球 Global & 6 \\
\hline I. D. Illar. & & Illarionova, Irina D. & 菊科 Asteraceae & 全球 Global & 5 \\
\hline M. C. Pace & & Pace, Matthew C. & 兰科 Orchidaceae & 全球 Global & 5 \\
\hline N. Kilian & & Kilian, Norbert & 菊科 Asteraceae & 全球 Global & 5 \\
\hline
\end{tabular}

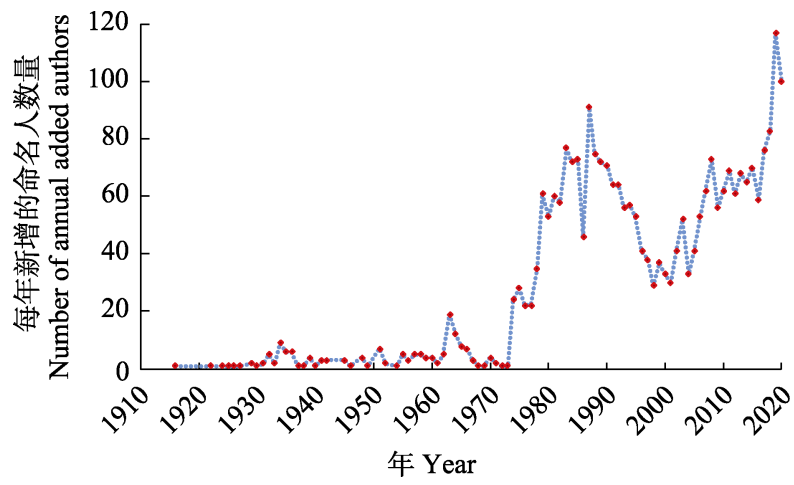

图2 1916年以来中国维管植物命名人年度新增人数

Fig. 2 Annual number of new added authors of Chinese vascular plants since 1916

菊科有 77 个植物名称被转移, 其中因为建立新 属而进行名称组合的有 13 个属的 64 个名称。兰科有 13 个植物名称被转移, 其中 2 个属的 5 个名称是因为 建立新属而进行的名称组合。

2020 年发表的 228 个种及种下等级新名称组合 由61个中外学者团队发表, 其中 36 个中国学者团队 发表了 67 个, 18 个国外学者团队发表了 133 个, 7 个 中外合作团队发表了 28 个。G. L. Nesom发表了 62 个 菊科植物组合, 全部是基于他本人建立的新属; C. B. Callaghan和Png团队发表了 25 个木兰科的新组合; Lowry和G. M. Plunkett团队发表了17个五加科的新
组合; 刘涁涁(B. B. Liu)和文军(J. Wen)团队发表了 16 个蓄薇科的植物新组合。发表 5 个以上新组合的 中外作者情况见表3。总体而言, 发表新组合的学者 一般都对某个类群进行过非常深入的研究, 发表名 称组合的学者数量显著少于发表新物种的学者。 2020 年参与命名新组合的中国学者有 65 人, 其中仅 10 人是 2020 年首次参与植物命名工作。

2020年有 85 个过去发表的植物名称被处理为 65 个名称的异名, 除去被处理为其他物种异名的物 种, 再排除中国分布的类群, 2020年中国高等植物 净增加数量为 294 个分类群, 占全国高等植物数量 的 $0.79 \%$ 。当年新发表的研究论文和专著中的名称 组合、新异名归并, 导致313个高等植物名称发生变 动, 占全国总数的 $0.84 \%$, 需要引起相关应用者和 研究人员的高度重视。

2020 年有 14 个曾经错误定名的物种重新被厘 定。这些名称订正一般都在植物名称的文献引证中 以固定格式出现, 一般不容易引起非专业人士的重 视。但这类订正对名称的正确应用有重要价值, 特 别是涉及到国家保护植物、珍稀濒危植物或有重要 经济价值植物的名实问题时尤需重视。例如红椿 (Toona ciliata M. Roem.) 属于国家二级保护野生植 
物, 继2017年湖北省第二次重点保护野生植物资源 调查中将野外的 “红椿” 定为红花香椿 (Toona fargesii A. Chev.), 2020年正式确认 《浙江植物志》 之前记载的“毛红椿” (Toona ciliata M. Roem. var. pubescens (Franch.) Hand.-Mazz.) 实为红花香椿之误 订(陈锋等, 2020)。

此外还有 147 个名称进行了后选模式和新模式 的指定。尽管这些模式化工作都是在分类学处理过 程中的附带工作, 但因其发表分散而很难完全被检 索。国际植物分类学会刊物Taxon在每一期的最后也 会在给出新物种和新名称索引外还给出当期所有 进行模式化物种的名称索引。我们也有必要对涉及 中国植物物种的模式化工作进行系统的整理。

值得一提的是2020年有2个物种被报道重新发 现。1920年发表的贝叶芒毛苣苔(Aeschynanthus monetaria Dunn)在发表后100年未被再次采集到, 曾经一度被认为仅存在于印度。 $\mathrm{Hu}$ 等(2020)重新采 集到新鲜材料, 并确认该物种只存在于中国西藏自 治区的墨脱县境内，纠正了产于印度的错误记录。 2001年发表的孟连苞叶兰 (Brachycorythis menglianensis Y. Y. Qian)发表后20年没有再次被采集到, 甚至被处理为长叶苞叶兰 (Brachycorythis henryi (Schltr.) Summerh.)的异名(Pedersen, 2010))。Wang YQ等(2020)重新采集到新鲜材料, 除了能够提供更 详细的研究信息外, 还确认了该物种应当给予种的 分类学地位。还有 2 个藓类植物门和 1 个苔类植物门 的物种排除了中国的分布, 此类报道在国内植物文 献中较少出现(Wang \& Jia, 2020; Bai et al, 2020)。

\section{发表中国高等植物名称论文的出版物}

发表有关于植物名称变化的出版物主要有期 刊和专著两种形式, 由于专著传播的速度一般慢于 期刊，不容易被及时索引，一般不作为物种发表的 主要媒介。2000年以来仅有19\%的植物名称发表在 专著中, 2020年发表在专著中的物种名称占比为 $21 \%$, 与历年数据基本持平。剩余 $79 \%$ 的物种名称则 发表在66种国际、国内期刊中。2020年涉及到高等 植物的新分类群(新属、新杂交属、新种、新杂交种、 新亚种、新变种、新变型)、新组合(等级)名称、新 名称、国家级新记录、新异名、后选(新)模式指定、 名称应用订正等资料的期刊论文有 405 篇。
发表中国植物新分类群论文的期刊比较集中。 统计发现，16种中外期刊包揽了 $85 \%$ 的文章，其余 50 种期刊只占 $15 \%$ 。2000年以来发表植物分类学相 关论文的中外期刊有 231 个，但绝大多数中国植物 名称相关的论文都发表在少数几个刊物上(图3)。 2020年有293篇涉及中国植物名称的研究论文发表 在被SCI收录的刊物上，占当年论文总数的近 $3 / 4$ 。 非SCI收录的中文刊物主要由中国学者发表, 主要 是新种下等级、国家级新记录、后选模式指定等; 西 文刊物主要由外国学者发表，一般是大篇幅的分类 学修订或者使用非英语作为主体语言。

发表新分类群最多的刊物是2009年创刊于新 西兰的Phytotaxa，其办刊宗旨就是快速发表植物新 分类群。该刊物2020年发表中国植物分类学相关的 论文128篇，包含 84 个新种。该刊物是新千年来发表 中国植物最多的期刊，至今已发表涉及中国植物类 群的论文794篇，发表新种589个。由于该刊物发表 周期短，发文量大，可以选择付费开放获取，已经 成为中国植物学者发表新分类群的重要平台。

2010年由Pensoft出版机构创办的开放获取刊 物PhytoKeys，自2018年以来受到了中国分类学者 的广泛关注。尽管文章出版需要数百欧元的处理费, 但2020年仍接受了 70篇涉及中国植物分类群的文 章, 其中包括59个新种。由于该刊物发表周期短, 发文量大, 成为中国植物学者发表新分类群的另一 个重要平台。

北欧传统分类学刊物Nordic Journal of Botany、 Annales Botanici Fennici和中国台湾的Taiwania等几 个历史悠久的分类学期刊，由于版面限制，涉及中 国分类群的文章数量保持在 20 篇左右。美国密苏里 植物园主办的植物分类学期刊Novon近年来更多关 注热带美洲和非洲的类群, 涉及中国植物类群的文 章较前 10 年明显减少。

中国本土的《广西植物》《热带亚热带植物学 报》《植物科学学报》《西北植物学报》《植物研究》 等植物学刊物近年来刊登的分类学文章数量明显 下降，其中发表的植物新分类群基本上是基于形态 描述，很少利用分子数据进行系统的研究。这些国 内刊物上刊登的分类学相关文章基本上是以报道 种下新分类群、国家级或省级新记录物种为主。

总而言之, 《中国植物志》和Flora of China的 编写完成并不代表中国植物家底全部清楚了。我们 


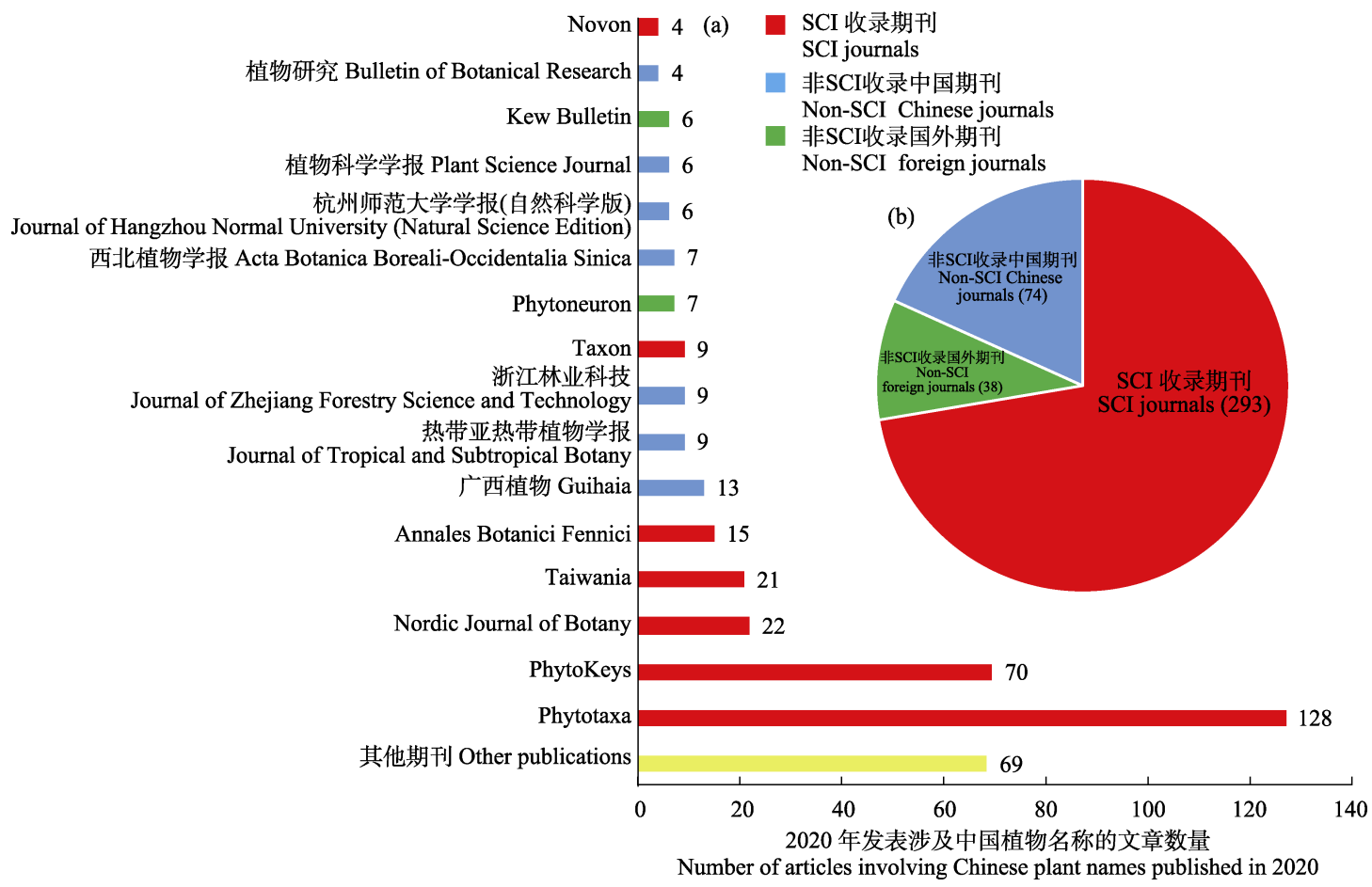

图32020年发表的涉及中国植物名称相关论文最多的16个期刊(a)及所有分类学论文发表刊物分类情况(b)

Fig. 3 Top 16 journals in which new names and name changes of Chinese vascular plants were published (a), and journals classification of all Chinese taxonomic articles (b) in 2020

的新种数据搜集整理工作表明, 中国植物仍然有许 多新物种有待发现。同时, 分子系统学研究的深入 带来的分类学修订也导致大量的名称出现变化。要 想完整地摸清中国植物的家底, 尚需相当时日。四 代植物学家 70 年持续接力编写纸质版植物志的时 代已经过去，现代可能更应该思考的是如何将动态 的植物信息完整、及时地展现在人们面前, 利用现 代信息技术手段编制新一代的可实时更新的数字 化植物志，则显得更加必要。

致谢: 本文原始文献查阅和获取得到了众多国内外 学者的支持, 特别是提供了很多国内机构没有订阅 的文献原文; 一些前辈学者得知我们在进行年度物 种整理时, 将所在机构发表的所有的新分类群发表 资料归类整理打包发送给我们，在此向这些前辈和 所有的支持者致以诚挚的谢意!

\section{参考文献}

Antonelli A, Fry C, Smith RJ, Simmonds MSJ, Kersey PJ, Pritchard HW, Abbo MS, Acedo C, Adams J, Ainsworth AM, and other 201 authors (2020) State of the World's Plants and Fungi 2020. Royal Botanic Gardens, Kew, London.
Bai Y, Qian K, Xiang YL, Yang ZW, Sulayman M, Li W, Shu L, Zhu RL (2020) Porella pinnata (Porellaceae) excluded from the liverwort flora of Asia. Nova Hedwigia, Beiheft 150, 193-200.

Bebber DP, Carine MA, Wood JRI, Wortley AH, Harris DJ, Prance GT, Davidse G, Paige J, Pennington TD, Robson NKB, Scotland RW (2010) Herbaria are a major frontier for species discovery. Proceedings of the National Academy of Sciences, USA, 107, 22169-22171.

Chen F, Xie WY, Zhang SL, Zhang FY, Chen ZH (2020) Additional notes on the seed plants in Zhejiang (II). Journal of Hangzhou Normal University (Natural Science Edition), 19, 96-102. (in Chinese with English abstract) [陈锋, 谢文 远, 张水利, 张芬耀, 陈征海 (2020) 浙江种子植物资料 订补(II). 杭州师范大学学报(自然科学版), 19, 96-102.]

Ding BY (2020) Flora of Zhejiang (new edition), Vol. 7. Zhejiang Science and Technology Press, Hangzhou. (in Chinese) [丁炳扬 (2020) 浙江植物志 (新编) 第七卷. 浙 江科学技术出版社, 杭州.]

Du C, Liao S, Boufford DE, Ma JS (2020) Twenty years of Chinese vascular plant novelties, 2000 through 2019. Plant Diversity, 42, 393-398.

Fontaine B, Perrard A, Bouchet P (2012) 21 years of shelf life between discovery and description of new species. Current Biology, 22, R943-R944.

Harber JF (2020) The Berberis of China and Vietnam, A revision. Monographs in Systematic Botany from the Missouri Botanical Garden, Vol. 136. Missouri Botanical 
Garden Press, St. Louis.

Hong DY, Blackmore S (2015) Plants of China: A Companion to the Flora of China. Cambridge University Press, Cambridge \& New York.

Hu J, Xiong YN, Li L, Liu Q, Wen F (2020) Rediscovery of Aeschynanthus monetaria (Gesneriaceae) in Southeast Tibet, China after more than 100 years. Phytotaxa, 450, 109-114.

Li L, Chung SW, Li B, Zeng SJ, Yan HF, Li SJ (2020) New insight into the molecular phylogeny of the genus Liparis s. l. (Orchidaceae: Malaxideae) with a new generic segregate: Blepharoglossum. Plant Systematics and Evolution, 306, 54.

Li YF, Zhang M, Wang XR, Sylvester SP, Xiang QB, Li X, Li M, Zhu H, Zhang C, Chen L, Yi XG, Mao LF, Duan YF (2020) Revisiting the phylogeny and taxonomy of Osmanthus (Oleaceae) including description of the new genus Chengiodendron. Phytotaxa, 436, 283-292.

Liu DK, Tu XD, Zhao Z, Zeng MY, Zhang S, Ma L, Zhang GQ, Wang MM, Liu ZJ, Lan SR, Li MH, Chen SP (2020) Plastid phylogenomic data yield new and robust insights into the phylogeny of Cleisostoma-Gastrochilus clades (Orchidaceae, Aeridinae). Molecular Phylogenetics and Evolution, 145, 106729.

Nesom GL (2020a) Yonglingia, Chaochienchangia, and Sinobouffordia (Asteraceae: Astereae), new genera segregated from Aster in China. Phytoneuron, 57, 1-25.

Nesom GL (2020b) Metamyriactis (Asteraceae, Astereae), a new genus of Southeast Asian Asters. Phytoneuron, 58, 1-36.

Nesom GL (2020c) Chlamydites (Asteraceae: Astereae) revived. Phytoneuron, 59, 1-14.

Nesom GL (2020d) Cordiofontis (Asteraceae: Astereae), a new genus of Himalayan species segregated from Aster. Phytoneuron, 60, 1-20.

Nesom GL (2020e) Cardiagyris (Asteraceae: Astereae), a new genus for the Doellingeria-like species of Asia. Phytoneuron, $61,1-26$.

Nesom GL (2020f) Tibetiodes Nesom, gen. nov. (Asteraceae: Astereae), including 27 species of Himalayan Aster. Phytoneuron, 63, 1-30.

Nesom GL (2020g) New genera from Asian Aster (Asteraceae: Astereae). Phytoneuron, 64, 1-44.

Pedersen HÆ (2010) Species delimitation and recognition in the Brachycorythis helferi complex (Orchidaceae) resolved by multivariate morphometric analysis. Botanical Journal of the Linnean Society, 162, 64-76.

Qin QM, Tong YH, Zheng XR, Ni JB, Xia NH (2020) Sinosasa (Poaceae: Bambusoideae), a new genus from China. Taxon, 70, 27-47.

Ren C, Wang L, Illarionova ID, Yang QE (2020) Circumscription and phylogenetic position of Ligularia sect.
Stenostegia (Asteraceae: Senecioneae) based on morphological, cytological, and molecular phylogenetic evidence. Taxon, 69, 739-755.

The Biodiversity Committee of Chinese Academy of Sciences (2020) Catalogue of Life China: 2020 Annual Checklist. Beijing. http://www.sp2000.org.cn/CoLChina/. (accessed on 2021-04-01)

Wang QH, Jia Y (2020) A taxonomic study of the genus Orthotrichum (Orthotrichaceae, Moss) in China. Acta Bryolichenologica Asiatica, 9, 19-166.

Wang YQ, Liu Q, Wu XF, Li JL, Wang BY, Pan B (2020) Rediscovery of Brachycorythis menglianensis (Orchidaceae) with additional descriptive notes. Guihaia, 40, 1623-1627.

Wang ZH, Kilian N, Chen YP, Peng H (2020) Sinoseris (Crepidinae, Cichorieae, Asteraceae), a new genus of three species endemic to China, one of them new to science. Willdenowia, 50, 91-110.

Willis KJ, Bachman S (2016) The State of the World's Plants Report-2016. Royal Botanic Gardens, Kew, London.

Willis KJ (2017) The State of the World's Plants Report-2017. Royal Botanic Gardens, Kew, London.

Zaika MA, Kilian N, Jones K, Krinitsina AA, Nilova MV, Speranskaya AS, Sukhorukov AP (2020) Scorzonera sensu lato (Asteraceae, Cichorieae)—Taxonomic reassessment in the light of new molecular phylogenetic and carpological analyses. PhytoKeys, 137, 1-85.

Zhang L, Zhou XM, Liang ZL, Fan XP, Lu NT, Song MS, Knapp R, Gao XF, Sun H, Zhang LB (2020a) Phylogeny and classification of the tribe Lepisoreae (Polypodiaceae; Pteridophyta) with the description of a new genus, Ellipinema gen. nov., segregated from Lepisorus. Molecular Phylogenetics and Evolution, 148, 106803.

Zhang L, Fan XP, Petchsri S, Zhou L, Pollawatn R, Zhang X, Zhou XM, Lu NT, Knapp R, Chantanaorrapint S, Limpanasittichai P, Sun H, Gao XF, Zhang LB (2020b) Evolutionary relationships of the ancient fern lineage the adder's tongues (Ophioglossaceae) with description of Sahashia gen. nov. Cladistics, 36, 380-393.

Zhang YX, Guo C, Li DZ (2020) A new subtribal classification of Arundinarieae (Poaceae, Bambusoideae) with the description of a new genus. Plant Diversity, 42, 127-134.

Zhang L, Zhang LB (2020a) ×Ellipisorus nom. nov. for $\times$ Lepinema (Polypodiaceae) and $\times$ Ellipisorus inaequibasis com. nov. from China. Phytotaxa, 464, 299-300.

Zhang L, Zhang LB (2020b) Fern nothogenera and $\times$ Lepinema, a new hybrid genus between Ellipinema and Lepisorus (Polypodiaceae). Phytotaxa, 455, 262-266.

(责任编委：陈又生 责任编辑：黄祥忠)

\section{附录 Supplementary Material}

\section{附录12020年中国植物新分类群文献目录}

Appendix 1 Bibliography of new taxa and name changes for Chinese plants in 2020 http://www.biodiversity-science.net/fileup/PDF/2021122-1.pdf 
杜诚, 刘军, 叶文, 廖帅, 葛斌杰, 刘冰, 马金双 (2021) 中国植物新分类群、新名称 2020 年度报告. 生物多样性, 29, 10111020. http://www.biodiversity-science.net/CN/10.17520/biods.2021122

附录12020年中国植物新分类群文献目录

Appendix 1 Bibliography of new taxa and name changes for Chinese plants in 2020

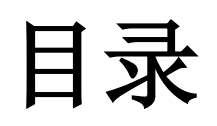

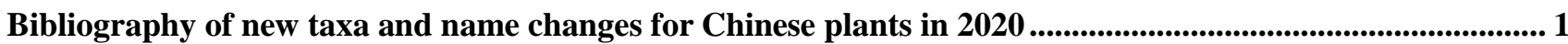

Acanthaceae 爵床科 ................................................... 1

Actinidiaceae 猕猴桃科 …………………………....... 1

Adoxaceae 五福花科.................................................. 1

Amaryllidaceae 石蒜科 ............................................. 2

Annonaceae 番荔枝科.................................................. 2

Apiaceae 企形科 ……………………………………... 2

Apocynaceae 夹竹桃科................................................. 3

Aquifoliaceae 冬青科................................................... 4

Araceae 天南星科 ………………………………….... 4

Araliaceae 五加科 …………………………………..... 5

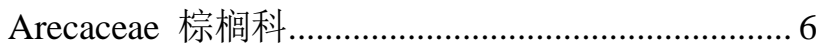

Aristolochiaceae 马兒铃科 ......................................... 6

Asparagaceae 天门冬科............................................... 7

Asphodelaceae 阿福花科 .............................................. 8

Aspleniaceae 铁角蕨科 ............................................... 8

Asteraceae 菊科............................................................... 8

Balsaminaceae 凤仙花科 ............................................ 21

Begoniaceae 秋海棠科............................................ 22

Berberidaceae 小檗科 .............................................. 24

Betulaceae 桦木科....................................................... 29

Blepharostomataceae 睫毛苔科.................................. 29

Boraginaceae 紫草科................................................2 29

Burmanniaceae 水玉䙃科 ....................................... 30

Buxaceae 黄杨科.................................................... 30

Cactaceae 仙人掌科...................................................... 30

Cannabaceae 大麻科 .................................................. 30

Caprifoliaceae 忍冬科............................................... 30

Caryophyllaceae 石竹科 ............................................. 30

Celastraceae 卫矛科 .................................................... 32

Combretaceae 使君子科 ............................................. 32

Commelinaceae 鸭跖草科........................................... 32

Cornaceae 山茱英科 ……………………………….... 32

Crassulaceae 景天科 ............................................... 32

Cucurbitaceae 葫芦科 ................................................. 33

Cupressaceae 柏科 .................................................... 33

Cyatheaceae 杪椤科 ............................................... 33

Cyperaceae 莎草科................................................... 33

Daphniphyllaceae 虎皮楠科 .................................... 34

Dryopteridaceae 鳞毛蕨科.......................................... 34

Encalyptaceae 大帽藓科 ............................................... 35

Ericaceae 杜鹃花科................................................... 35

Euphorbiaceae 大戟科............................................... 35

Fabaceae 豆科 …………………………………...... 36

Fagaceae 壳斗科 .................................................. 38
Fissidentaceae 凤尾藓科...........................................38

Funariaceae 葫芦藓科................................................38

Gentianaceae 龙胆科 ................................................38

Gesneriaceae 苦苣苔科...............................................39

Gnetaceae 买麻藤科 ………………………………......43

Grimmiaceae 紫萝藓科 ...............................................44

Grossulariaceae 茶蔍子科 ..............................................4

Hydrangeaceae 绣球花科 ...........................................4

Hymenophyllaceae 膜薜科 ..........................................4

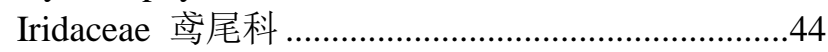

Lamiaceae 唇形科......................................................4

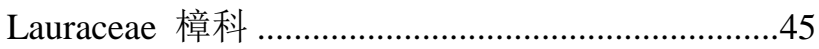

Lejeuneaceae 细鳞苔科 ...............................................45

Liliaceae 百合科 ………………………………......46

Loganiaceae 马钱科......................................................46

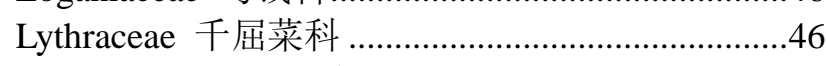

Magnoliaceae 木兰科......................................................4

Malvaceae 锦葵科......................................................51

Marattiaceae 合囊顸科 ...............................................51

Mayacaceae 花水藓科 .................................................52

Melanthiaceae 菉芦科.................................................52

Melastomataceae 野牡丹科 .......................................52

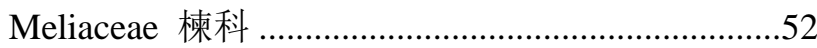

Meteoriaceae 蔓藓科 ............................................52

Mniaceae 提灯藓科 …………………………………....53

Moraceae 桑科 …………………………………....53

Myrtaceae 桃金娘科 .................................................53

Oleaceae 木樨科 ......................................................54

Onagraceae 柳叶菜科....................................................55

Ophioglossaceae 瓶尔小草科......................................55

Orchidaceae 兰科............................................................55

Orthotrichaceae 木灵藓科 .........................................60

Orobanchaceae 列当科 ..........................................61

Oxalidaceae 酢浆草科 ................................................62

Papaveraceae 罂粟科 ……………………………........63

Pentaphylacaceae 五列木科......................................63

Phyllanthaceae 叶下珠科.........................................63

Phytolaccaceae 商陆科 .............................................64

Pinaceae 松科............................................................64

Piperaceae 胡椒科......................................................64

Plagiochilaceae 羽苔科............................................64

Poaceae 禾本科 ......................................................64

Polygonaceae 蓼科......................................................67

Polypodiaceae 水龙骨科............................................67 
杜诚, 刘军, 叶文, 廖帅, 葛斌杰, 刘冰, 马金双 (2021) 中国植物新分类群、新名称 2020 年度报告. 生物多样性, 29, 10111020. http://www.biodiversity-science.net/CN/10.17520/biods.2021122

Porellaceae 光䒓苔科 68

Saxifragaceae 虎耳草科 .79

Primulaceae 报春花科.............................................68

Scapaniaceae 合叶苔科 ..........................................79

Pteridaceae 凤尾蕨科.............................................6 68

Selaginellaceae 卷柏科 ...........................................79

Ranunculaceae 毛茛科 ........................................... 69

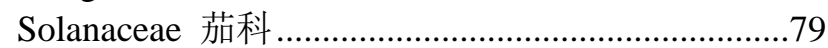

Resedaceae 木樨草科............................................... 71

Rhamnaceae 鼠李科................................................ 71

Styracaceae 安息香科..............................................79

Rosaceae 蓠薇科 .................................................... 71

Rubiaceae 茜草科................................................... 77

Rutaceae 芸香科...................................................... 77

Salicaceae 杨柳科 ................................................... 77

List of journals.

Theaceae 山茶科 ...................................................8 80

Thymelaeaceae 瑞香科 .............................................81

Vitaceae 葡萄科 ....................................................81

Woodsiaceae 岩蕨科 ................................................81

Zingiberaceae 姜科 ................................................81

签。 


\title{
Bibliography of new taxa and name changes for Chinese plants in 2020
}

\author{
Acanthaceae 爵床科 \\ Eranthemum macrophyllum Wall. ex Nees 大叶可爱花 [New Record] \\ GONG Qiang-bang, MA Xing-da, YIN Hai-jun, WANG Wen-guang, DUAN Chun-cheng, DENG Yun-fei, SHI Gan-mo, SHEN Jian- \\ yong, Eranthemum macrophyllum Wall. ex Nees (Acanthaceae), a Newly Recorded Species from China. Journal of Tropical and \\ Subtropical Botany 28 (6): 624-627. 2020 [625] doi: 10.11926/jtsb.4191 \\ CHINA (Yunnan); MYANMAR* \\ Eranthemum macrophyllum Wall. ex Nees 大叶可爱花 [Typification] \\ GONG Qiang-bang, MA Xing-da, YIN Hai-jun, WANG Wen-guang, DUAN Chun-cheng, DENG Yun-fei, SHI Gan-mo, SHEN Jian- \\ yong, Eranthemum macrophyllum Wall. ex Nees (Acanthaceae), a Newly Recorded Species from China. Journal of Tropical and \\ Subtropical Botany 28 (6): 624-627. 2020 [625] doi: 10.11926/jtsb.4191
}

CHINA (Yunnan); MYANMAR*

Gymnostachyum morsei Y. F. Deng 龙州裸柱草 [New Species] IUCN: CR: B2a, b(i); Evidence: Morphology

DENG Yun-fei, TAN Yun-hong, LIN Zhe-li, HUANG Yun-feng, Gymnostachyum morsei (Acanthaceae: Andrographideae), a New Species from Guangxi, China. Kew Bulletin 75 (59): 1-6. 2020 [2] doi: 10.1007/S12225-020-09920-5

CHINA (Guangxi*)

Pseuderanthemum album (Nees) Radlk. [Typification]

BORAH Dipankar, SINGH Rajeev Kumar, TARAM Momang, Pseuderanthemum arunachalense, a New Species from Eastern Himalaya, India and Typification of Some Names in Pseuderanthemum (Acanthaceae). Indian Forester 146 (7): 660-664. 2020 [662] doi: 10.36808/if/2020/v146i7/149848

CHINA (Xizang); BANGLADESH, INDIA, MYANMAR

Pseuderanthemum arunachalense D. Borah, R. Kr. Singh \& Taram [New Species] IUCN: NE; Evidence: Morphology

BORAH Dipankar, SINGH Rajeev Kumar, TARAM Momang, Pseuderanthemum arunachalense, a New Species from Eastern Himalaya, India and Typification of Some Names in Pseuderanthemum (Acanthaceae). Indian Forester 146 (7): 660-664. 2020 [660] doi: 10.36808/if/2020/v146i7/149848

CHINA (Xizang*)

Pseuderanthemum leptanthus (C. B. Clarke) Lindau [Typification]

BORAH Dipankar, SINGH Rajeev Kumar, TARAM Momang, Pseuderanthemum arunachalense, a New Species from Eastern Himalaya, India and Typification of Some Names in Pseuderanthemum (Acanthaceae). Indian Forester 146 (7): 660-664. 2020 [662] doi: 10.36808/if/2020/v146i7/149848

CHINA (Xizang*); MYANMAR

Rungia stolonifera C. B. Clarke [New Synomyms]

S.: Rungia axilliflora H. S. Lo; Rungia densiflora H. S. Lo; Rungia evrardii Benoist

LIN Zhe-li, HAI Do Van, DENG Yun-fei, Three New Synonyms of Rungia stolonifera (Acanthaceae) from China and Vietnam. Nordic Journal of Botany 38 (e02649): 1-5. 2020 [3] doi: 10.1111/njb.02649

CHINA (Anhui, Guangdong, Guangxi, Guizhou, Jiangxi, Yunnan, Zhejiang); INDIA*, THAILAND, VIET NAM

Strobilanthes sunhangii T. Deng, J. T. Chen \& Y. F. Deng [New Species] IUCN: DD; Evidence: Morphology

CHEN Jun-tong, HUANG Xian-han, LV Zhen-yu, KUANG Tian-hui, LUO Jian, DENG Yun-fei, DENG Tao, Strobilanthes sunhangii

(Acanthaceae), a New Species from Tibet, China. PhytoKeys 166: 117-127. 2020 [119] doi: 10.3897/phytokeys.166.58831 CHINA (Xizang*)

\section{Actinidiaceae 猕猴桃科}

Actinidia macrosperma C. F. Liang 大籽猕猴桃 [New Synonyms]

S.: Actinidia valvata Dunn. var. longipedicellata L. L. Yu

CHEN Feng, XIE Wen-yuan, ZHANG Fen-yao, ZHANG Hong-wei, CHEN Xian-xing, CHEN Zheng-hai, Notes on Seed Plant in Zhejiang Province (V). Journal of Zhejiang Forestry Science and Technology 40 (4): 67-72. 2020 [68] doi: 10.3969/j.issn.10013776.2020.04.012

CHINA (Zhejiang*)

\section{Adoxaceae 五福花科}


杜诚, 刘军, 叶文, 廖帅, 葛斌杰, 刘冰, 马金双 (2021) 中国植物新分类群、新名称 2020 年度报告. 生物多样性, 29, 10111020. http://www.biodiversity-science.net/CN/10.17520/biods.2021122

Viburnum fengyangshanense Z. H. Chen, P. L. Chiu \& L. X. Ye 凤阳山荚蒾 [New Species] IUCN: NE; Evidence: Morphology QIU Bao-lin, YE Li-xin, CHEN Feng, XIE Wen-yuan,CHEN Zheng-hai, Additional Notes on Viburnum L. in Zhejiang. Journal of Hangzhou Normal University (Natural Science Edition) 19 (3): 261-266. 2020 [261] doi: 10.12191/j.issn.1674-232X.2020.03.008 CHINA (Zhejiang*)

Viburnum thunbergianum Z. H. Chen \& P. L. Chiu 蝴蝶荚蒾 [New Name]

R.: Viburnum tomentosum Thunb.

QIU Bao-lin, YE Li-xin, CHEN Feng, XIE Wen-yuan,CHEN Zheng-hai, Additional Notes on Viburnum L. in Zhejiang. Journal of Hangzhou Normal University (Natural Science Edition) 19 (3): 261-266. 2020 [261] doi: 10.12191/j.issn. 1674-232X.2020.03.008 CHINA (Zhejiang)

\section{Amaryllidaceae 石蒜科}

Allium xinlongense D. F. Xie \& X. J. He [New Species] IUCN: NE; Evidence: ITS, rpL32-trnL; Morphology

XIE Deng-feng, XIE Fu-min, JIA Sheng-bin, LI Hao, YANG Xin, ZHANG Xiang-yi, ZHOU Song-dong, HE Xing-jin, Allium xinlongense (Amaryllidaceae, Allioideae), a New Species from Western Sichuan. Phytotaxa 432 (3): 274-282. 2020 [280] doi: 10.11646/phytotaxa.432.3.4

CHINA (Sichuan*)

Lycoris tsinlingensis P. C. Zhang, Yi Jun Lu \& Ting Wang bis [New Species] IUCN: NE; Evidence: Morphology

LU Yi-jun, WANG, Ting, WANG Yi-chen, ZHANG, Peng-chong, Lycoris tsinlingensis (Amaryllidaceae), a New Species from Shaanxi, China. Annales Botanici Fennici 57 (4-6): 193-196. 2020 [193] doi: 10.5735/085.057.0424

CHINA (Shaanxi*)

\section{Annonaceae 番荔枝科}

Polyalthiopsis chinensis (S. K. Wu ex P. T. Li) B. Xue \& Y. H. Tan 西藏疮叶木 [New Combination]

B.: Polyalthia chinensis S. K. Wu \& P. T. Li

XUE Bin-e, DING Hong-bo, YAO Gang, SHAO Yun-yun, FAN Xiao-jing, TAN Yun-hong, From Polyalthia to Polyalthiopsis (Annonaceae): Transfer of Species Enlarges a Previously Monotypic Genus. PhytoKeys 148: 71-91. 2020 [82] doi: 10.3897/phytokeys.148.50929

CHINA (Xizang*)

Polyalthiopsis verrucipes (C. Y. Wu ex P. T. Li) B. Xue \& Y. H. Tan 疮叶木 [New Combination]

B.: Polyalthia verrucipes C. Y. Wu ex P. T. Li

XUE Bin-e, DING Hong-bo, YAO Gang, SHAO Yun-yun, FAN Xiao-jing, TAN Yun-hong, From Polyalthia to Polyalthiopsis (Annonaceae): Transfer of Species Enlarges a Previously Monotypic Genus. PhytoKeys 148: 71-91. 2020 [82] doi: 10.3897/phytokeys.148.50929

CHINA (Yunnan*)

Polyalthiopsis verrucipes (C. Y. Wu ex P. T. Li) B. Xue \& Y. H. Tan 疮叶木 [Typification]

XUE Bin-e, DING Hong-bo, YAO Gang, SHAO Yun-yun, FAN Xiao-jing, TAN Yun-hong, From Polyalthia to Polyalthiopsis (Annonaceae): Transfer of Species Enlarges a Previously Monotypic Genus. PhytoKeys 148: 71-91. 2020 [82] doi: 10.3897/phytokeys.148.50929

CHINA (Yunnan*)

\section{Apiaceae 伞形科}

Angelica yanyuanensis (F. T. Pu) Jing Zhou [New Combination]

B.: Ligusticum yanyuanense F. T. Pu

ZHOU Jing, GAO Yu-zhen, WEI Jin, LIU Zhen-wen, DOWNIE Stephen R., Molecular Phylogenetics of Ligusticum (Apiaceae) Based on Nrdna Its Sequences: Rampant Polyphyly, Placement of the Chinese Endemic Species, and a Much-Reduced Circumscription of the Genus. International Journal of Plant Sciences 181 (3): 306-323. 2020 [321] doi: 10.1086/706851

CHINA (Sichuan*)

Cuminum borszczowii (Regel \& Schmalh.) Koso-Pol. 长毛孜然芹 [New Record]

QIU Juan, LI Wen-jun, YANG Zong-zong, TAN Dun-yan, Cuminum borszczowii (Regel et Schmalh.) Koso-Pol., a Newly Recorded Species of Apiaceae from China. Journal of Tropical and Subtropical Botany 28 (1): 101-104. 2020 [102] doi: 10.11926/jtsb.4084 CHINA (Xinjiang); KAZAKHSTAN*

Hymenidium apiolens (C. B. Clarke) Pimenov \& Kljuykov [New Synomyms]

S.: Hymenidium pilosum (C. B. Clarke ex H. Wolff) Pimenov \& Kljuykov; Pleurospermum pilosum C. B. Clarke ex H. Wolff

GUO Xian-lin, GOU Wei, XIE Fu-min, ZHOU Song-dong, HE Xing-jin, Hymenidium pilosum (Apiaceae) Is a Synonym of H. apiolens Based on Morphology and Molecular Data. Nordic Journal of Botany 38 (e02864): 1-9. 2020 [7] doi: 10.1111/njb.02864 
杜诚, 刘军, 叶文, 廖帅, 葛斌杰, 刘冰, 马金双 (2021) 中国植物新分类群、新名称 2020 年度报告. 生物多样性, 29, 10111020. http://www.biodiversity-science.net/CN/10.17520/biods.2021122

CHINA (Xizang); INDIA*, NEPAL

Hymenidium dentatum (Wall. ex DC.) Pimenov \& Kljuykov [New Record]

GUO Xian-lin, GOU Wei, PENG Chang, ZHOU Song-dong, HE Xing-jin, New insights into the Phylogenetic Position of Hymenidium dentatum (Apioideae, Apiaceae) Inferred from nrDNA and Morphological Evidence. Phytotaxa 452 (1): 46-54. 2020 [52] doi: 10.11646/phytotaxa.452.1.4

CHINA (Xizang); NEPAL*

Ligusticum litangense F. T. Pu [New Synonyms]

S.: Hansenia forrestii (H. Wolff) Pimenov \& Kljuykov

ZHOU Jing, GAO Yu-zhen, WEI Jin, LIU Zhen-wen, DOWNIE Stephen R., Molecular Phylogenetics of Ligusticum (Apiaceae) Based on Nrdna Its Sequences: Rampant Polyphyly, Placement of the Chinese Endemic Species, and a Much-Reduced Circumscription of the Genus. International Journal of Plant Sciences 181 (3): 306-323. 2020 [321] doi: 10.1086/706851

CHINA (Sichuan*)

Meeboldia linearis X. L. Guo, W. Gou \& X. J. He 线叶滇芹 [New Species] IUCN: DD; Evidence: ITS; Morphology

GUO Xian-lin, GOU Wei, XIE Fu-min, ZHOU Song-dong, HE Xing-jin, Meeboldia linearis sp. nov. (Apiaceae) from Xizang, China. Nordic Journal of Botany 38 (e02635): 1-7. 2020 [6] doi: 10.1111/njb.02635

CHINA (Xizang*)

Peucedanum huangshanense Lu Q. Huang, H. S. Peng \& S. S. Chu [New Species] IUCN: NE; Evidence: ITS; Morphology

CHU Shan-shan, WANG De-qun, PENG Hua-sheng, HUANG Lu-qi, Peucedanum huangshanense (Apiaceae), a New Species from Anhui, China. Phytotaxa 430 (1): 17-24. 2020 [21] doi: 10.11646/phytotaxa.430.1.3

CHINA (Anhui*)

Pleurospermopsis bicolor (Franch.) Jing Zhou \& J. Wei [New Combination]

B.: Pleurospermum govanianum Benth. ex C. B. Clarke var. bicolor Franch.

ZHOU Jing, WEI Jin, LIU Zhen-wen, An Expanded Circumscription for the Previously Monotypic Pleurospermopsis (Apiaceae) Based on nrDNA ITS Sequences and Morphological Evidence. Phytotaxa 460 (2): 129-136. 2020 [131] doi: 10.11646/phytotaxa.460.2.2

CHINA (Yunnan*); BHUTAN, INDIA

Pternopetalum paucifoliolatum J. F. Ye, X. Jie Li \& Ce H. Li 少羽囊瓣芹 [New Species] IUCN: CR: C2a(ii); Evidence: Morphology YE Jian-fei, CHEN Liang, SHAN Zhang-jian, LI Xiao-jie, LI Ce-hong, Pternopetalum paucifoliolatum (Apiaceae), a New Critically Endangered Species from Sichuan, China. PhytoKeys 166: 97-103. 2020 [99] doi: 10.3897/phytokeys. 166.54646

CHINA (Sichuan*)

Tetrataenium wallichii (DC.) Manden. 须弥四带芹 [New Record]

LIU Cheng, YA Ji-dong, GUO Yong-jie, CAI Jie, ZHANG Ting, Newly recorded species of seed plants from Xizang, China. Biodiversity Science 28 (10): 1238-1245. 2020 [1239] doi: 10.17520/biods.2020161

CHINA (Xizang); BUTAN, INDIA, NEPAL*

Tongoloa arguta L. J. Gui \& X. J. He [New Species] IUCN: NT; Evidence: ITS; Morphology

GUI Ling-jian, WEN Jun, XIAO Yan-ping, REN Ting, ZHENG Hong-yi, HE Xing-jin, Tongoloa arguta (Apiaceae), a New Species from Southwest China. PhytoKeys 164: 11-19. 2020 [14] doi: 10.3897/phytokeys.164.54927

CHINA (Sichuan*)

Tongoloa tagongensis L. J. Gui \& X. J. He 塔公东俄芹 [New Species] IUCN: NE; Evidence: ITS; Morphology

GUI Ling-jian, JIA Sheng-bin, GUO Xian-lin, PRICE Megan, ZHOU Song-dong, HE Xing-jin, Tongoloa tagongensis (Apiaceae), a New Species from the Hengduan Mountains, China. Phytotaxa 461 (1): 12-20. 2020 [17] doi: 10.11646/phytotaxa.461.1.2 CHINA (Sichuan*)

\section{Apocynaceae 夹竹桃科}

Hoya gaoligongensis M. X. Zhao \& Y. H. Tan 高黎贡球兰 [New Species] IUCN: VU; Evidence: Morphology

ZHAO Ming-Xu, WANG Heng-Ying, YIN Zhi-Jian, WANG Meng-Jun, YANG Jin-Chao, TAN Yun-Hong, Hoya gaoligongensis (Apocynaceae, Asclepiadoideae), a New Species from Yunnan, SW China. Phytotaxa 459 (3): 219-226. 2020 [220] doi: 10.11646/phytotaxa.459.3.3

CHINA (Yunnan*)

Hoya longicalyx Hui Wang bis \& E. F. Huang 长萼球兰 [New Species] IUCN: DD; Evidence: ITS; Morphology

HUANG Er-feng, YANG Lei-lei, XI Wang, LIU Yi-ye, Hui WANG Hui, Hoya longicalyx, a New Species of Hoya (Apocynaceae: Asclepiadoideae) from Yunnan, China. Taiwania 65 (3): 353-359. 2020 [354] doi: 10.6165/tai.2020.65.353

CHINA (Yunnan*) 
杜诚, 刘军, 叶文, 廖帅, 葛斌杰, 刘冰, 马金双 (2021) 中国植物新分类群、新名称 2020 年度报告. 生物多样性, 29, 10111020. http://www.biodiversity-science.net/CN/10.17520/biods.2021122

Hoya nyingchiensis Y. W. Zuo \& H. P. Deng [New Species] IUCN: NE; Evidence: Morphology

ZUO You-wei, LAN Xiao-zhong, DENG Hong-ping, ZHAO Fang-yu, YUAN Xiao-feng, Hoya nyingchiensis (Apocynaceae, Asclepiadoideae), a New Species from Tibet, China. Phytotaxa 468 (1): 130-136. 2020 [130] doi: 10.11646/phytotaxa.468.1.9

CHINA (Xizang*)

\section{Aquifoliaceae 冬青科}

Ilex fargesii Franch. subsp. fargesii [New Synonyms]

S.: Ilex fargesii Franch. var. angustifolia Z. Ying Zhang; Ilex fargesii Franch. var. brevifolia S. Andrews

YANG Yi, CHEN Li, SUN Lu, PENG Hua, Taxonomic Notes on Ilex sect. Ilex (Aquifoliaceae) from China II: Revision of I. fargesii and Related Species Based on Molecular and Morphological Evidence. Phytotaxa 432 (1): 47-64. 2020 [55] doi: 10.11646/phytotaxa.432.1.5

CHINA (Chongqing*, Gansu, Hubei, Hunan, Guizhou, Shaanxi, Sichuan, Yunnan)

Ilex jiangmenensis Lei Jiang \& K. W. Xu 江门冬青 [New Species] IUCN: NE; Evidence: Morphology

JIANG Lei, WU Lin-fang, HUANG Guan-yun, YU Jia-rong, HE BI-SHENG, XU Ke-wang, A New Species of Ilex sect. Ilex (Aquifoliaceae) from Guangdong, China. Phytotaxa 428 (2): 153-158. 2020 [153] doi: 10.11646/phytotaxa.428.2.10

CHINA (Guangdong*)

Ilex wattii Loes. [Typification]

YANG Yi, CHEN Li, SUN Lu, PENG Hua, Taxonomic Notes on Ilex sect. Ilex (Aquifoliaceae) from China II: Revision of I. fargesii and Related Species Based on Molecular and Morphological Evidence. Phytotaxa 432 (1): 47-64. 2020 [60] doi: 10.11646/phytotaxa.432.1.5

CHINA (Yunnan); INDIA*

Ilex wattii Loes. subsp. marlipoensis (H. W. Li ex Y. R. Li) Yi Yang \& H. Peng [New Combination]

B.: Ilex marlipoensis H. W. Li ex Y. R. Li

YANG Yi, CHEN Li, SUN Lu, PENG Hua, Taxonomic Notes on Ilex sect. Ilex (Aquifoliaceae) from China II: Revision of I. fargesii and Related Species Based on Molecular and Morphological Evidence. Phytotaxa 432 (1): 47-64. 2020 [60] doi: 10.11646/phytotaxa.432.1.5

CHINA (Yunnan*)

Ilex wattii Loes. subsp. marlipoensis (H. W. Li ex Y. R. Li) Yi Yang \& H. Peng [New Synonyms]

S.: Ilex cupreonitens C. Y. Wu ex Y. R. Li

YANG Yi, CHEN Li, SUN Lu, PENG Hua, Taxonomic Notes on Ilex sect. Ilex (Aquifoliaceae) from China II: Revision of I. fargesii and Related Species Based on Molecular and Morphological Evidence. Phytotaxa 432 (1): 47-64. 2020 [60] doi: 10.11646/phytotaxa.432.1.5

CHINA (Yunnan*)

Ilex wattii Loes. subsp. wattii [New Synonyms]

S.: Ilex gintungensis H. W. Li ex Y. R. Li; Ilex venosa C. Y. Wu ex Y. R. Li

YANG Yi, CHEN Li, SUN Lu, PENG Hua, Taxonomic Notes on Ilex sect. Ilex (Aquifoliaceae) from China II: Revision of I. fargesii and Related Species Based on Molecular and Morphological Evidence. Phytotaxa 432 (1): 47-64. 2020 [60] doi: 10.11646/phytotaxa.432.1.5

CHINA (Yunnan); INDIA*

\section{Araceae 天南星科}

Alocasia lihengiae C. L. Long \& Q. Fang [New Species] IUCN: NE; Evidence: Morphology

FANG Qiong, YANG Jun, LONG Chun-lin, Alocasia lihengiae, a New Species of Araceae from Southern Yunnan. Phytotaxa 436 (2): 97-103. 2020 [98] doi: 10.11646/phytotaxa.436.2.1

CHINA (Yunnan*)

Alocasia yunqiana Z. X. Ma, Yi F. Li \& J. T. Yin 运气海芋 [New Species] IUCN: NE; Evidence: Morphology

MA Zheng-xu, LI Yi-fan, DAO Bao-hui, YANG Wan-liu, LIU Bing, YIN Jian-tao, Taxonomic Notes on the Alocasia-Colocasia Clade (Araceae) in China I: Alocasia yunqiana, a New Species from Tongbiguan Nature Reserve, Yunnan Province. Phytotaxa 460 (4): 277-284. 2020 [278] doi: 10.11646/phytotaxa.460.4.5

CHINA (Yunnan*)

Arisaema bockii Engl. f. viridescens (D. D. Ma) W. Y. Xie \& B. Y. Ding 绿苞灯台莲 [New Combination]

B.: Arisaema sikokianum Franch. \& Sav. var. viridescens D. D. Ma

CHEN Feng, XIE Wen-yuan, ZHANG Fen-yao, ZHANG Hong-wei, CHEN Xian-xing, CHEN Zheng-hai, Notes on Seed Plant in Zhejiang Province (V). Journal of Zhejiang Forestry Science and Technology 40 (4): 67-72. 2020 [69] doi: 10.3969/j.issn.10013776.2020.04.012 
杜诚, 刘军, 叶文, 廖帅, 葛斌杰, 刘冰, 马金双 (2021) 中国植物新分类群、新名称 2020 年度报告. 生物多样性, 29, 10111020. http://www.biodiversity-science.net/CN/10.17520/biods.2021122

CHINA (Zhejiang*)

\section{Araliaceae 五加科}

Eleutherococcus nodiflorus (Dunn) S. Y. Hu var. trifoliolatus (C. B. Shang) Shui L. Zhang \& Z. H. Chen 三叶五加 [New Combination] B.: Acanthopanax gracilistylus W. W. Sm. var. trifoliolatus C. B. Shang

CHEN Feng, XIE Wen-yuan, ZHANG Shui-li, ZHANG Fen-yao, CHEN Zheng-hai, Additional Notes on the Seed Plants in Zhejiang (II). Journal of Hangzhou Normal University (Natural Science Edition) 19 (6): 96-102. 2020 [98] doi: 10.12191/j.issn.1674232X.2020.06.014

CHINA (Zhejiang*)

Heptapleurum brevipedicellatum (Harms) Lowry \& G. M. Plunkett [New Combination]

B.: Schefflera brevipedicellata Harms

LOWRY Porter P., PLUNKETT Gregory M., Resurrection of the Genus Heptapleurum for the Asian Clade of Species Previously Included in Schefflera (Araliaceae). Novon 28 (3): 143-170. 2020 [148] doi: 10.3417/2020612

CHINA (Yunnan); VIET NAM

Heptapleurum chapanum (Harms) Lowry \& G. M. Plunkett [New Combination]

B.: Schefflera chapana Harms

LOWRY Porter P., PLUNKETT Gregory M., Resurrection of the Genus Heptapleurum for the Asian Clade of Species Previously Included in Schefflera (Araliaceae). Novon 28 (3): 143-170. 2020 [150] doi: 10.3417/2020612

CHINA (Yunnan); VIET NAM

Heptapleurum fengii (C. J. Tseng \& G. Hoo) Lowry \& G. M. Plunkett [New Combination]

B.: Schefflera fengii C. J. Tseng \& G. Hoo

LOWRY Porter P., PLUNKETT Gregory M., Resurrection of the Genus Heptapleurum for the Asian Clade of Species Previously Included in Schefflera (Araliaceae). Novon 28 (3): 143-170. 2020 [153] doi: 10.3417/2020612

CHINA (Yunnan)

Heptapleurum glabrescens (C. J. Tseng \& G. Hoo) Lowry \& G. M. Plunkett [New Combination]

B.: Schefflera impressa (C. B. Clarke) Harms var. glabrescens C. J. Tseng \& G. Hoo

LOWRY Porter P., PLUNKETT Gregory M., Resurrection of the Genus Heptapleurum for the Asian Clade of Species Previously Included in Schefflera (Araliaceae). Novon 28 (3): 143-170. 2020 [153] doi: 10.3417/2020612

CHINA (Xizang, Yunnan); MYANMAR

Heptapleurum guizhouense (C. B. Shang) Lowry \& G. M. Plunkett [New Combination]

B.: Schefflera guizhouensis C. B. Shang

LOWRY Porter P., PLUNKETT Gregory M., Resurrection of the Genus Heptapleurum for the Asian Clade of Species Previously Included in Schefflera (Araliaceae). Novon 28 (3): 143-170. 2020 [154] doi: 10.3417/2020612

CHINA (Guizhou)

Heptapleurum hainanense (Merr. \& Chun) Lowry \& G. M. Plunkett [New Combination]

B.: Schefflera hainanensis Merr. \& Chun

LOWRY Porter P., PLUNKETT Gregory M., Resurrection of the Genus Heptapleurum for the Asian Clade of Species Previously Included in Schefflera (Araliaceae). Novon 28 (3): 143-170. 2020 [154] doi: 10.3417/2020612

CHINA (Hainan); VIET NAM

Heptapleurum hypoleucoides (Harms) Lowry \& G. M. Plunkett [New Combination]

B.: Schefflera hypoleucoides Harms

LOWRY Porter P., PLUNKETT Gregory M., Resurrection of the Genus Heptapleurum for the Asian Clade of Species Previously Included in Schefflera (Araliaceae). Novon 28 (3): 143-170. 2020 [155] doi: 10.3417/2020612

CHINA (Yunnan); THAILAND, VIET NAM

Heptapleurum insigne (C. N. Ho) Lowry \& G. M. Plunkett [New Combination]

B.: Schefflera insignis C. N. Ho

LOWRY Porter P., PLUNKETT Gregory M., Resurrection of the Genus Heptapleurum for the Asian Clade of Species Previously Included in Schefflera (Araliaceae). Novon 28 (3): 143-170. 2020 [155] doi: 10.3417/2020612

CHINA (Guangdong)

Heptapleurum marlipoense (C. J. Tseng \& G. Hoo) Lowry \& G. M. Plunkett [New Combination]

B.: Schefflera marlipoensis C. J. Tseng \& G. Hoo

LOWRY Porter P., PLUNKETT Gregory M., Resurrection of the Genus Heptapleurum for the Asian Clade of Species Previously Included in Schefflera (Araliaceae). Novon 28 (3): 143-170. 2020 [159] doi: 10.3417/2020612 
杜诚, 刘军, 叶文, 廖帅, 葛斌杰, 刘冰, 马金双 (2021) 中国植物新分类群、新名称 2020 年度报告. 生物多样性, 29, 10111020. http://www.biodiversity-science.net/CN/10.17520/biods.2021122

\section{CHINA (Yunnan)}

Heptapleurum metcalfianum (Merr. ex H. L. Li) Lowry \& G. M. Plunkett [New Combination] B.: Schefflera metcalfiana Merr. ex H. L. Li

LOWRY Porter P., PLUNKETT Gregory M., Resurrection of the Genus Heptapleurum for the Asian Clade of Species Previously Included in Schefflera (Araliaceae). Novon 28 (3): 143-170. 2020 [159] doi: 10.3417/2020612 CHINA (Yunnan)

Heptapleurum multinervium (H. L. Li) Lowry \& G. M. Plunkett [New Combination]

B.: Schefflera multinervia H. L. Li

LOWRY Porter P., PLUNKETT Gregory M., Resurrection of the Genus Heptapleurum for the Asian Clade of Species Previously Included in Schefflera (Araliaceae). Novon 28 (3): 143-170. 2020 [160] doi: 10.3417/2020612

CHINA (Yunnan)

Heptapleurum napuoense (C. B. Shang) Lowry \& G. M. Plunkett [New Combination]

B.: Schefflera napuoensis C. B. Shang

LOWRY Porter P., PLUNKETT Gregory M., Resurrection of the Genus Heptapleurum for the Asian Clade of Species Previously Included in Schefflera (Araliaceae). Novon 28 (3): 143-170. 2020 [160] doi: 10.3417/2020612

CHINA (Guangxi)

Heptapleurum petelotii (Merr.) Lowry \& G. M. Plunkett [New Combination]

B.: Schefflera petelotii Merr.

LOWRY Porter P., PLUNKETT Gregory M., Resurrection of the Genus Heptapleurum for the Asian Clade of Species Previously Included in Schefflera (Araliaceae). Novon 28 (3): 143-170. 2020 [162] doi: 10.3417/2020612

CHINA (Yunnan)

Heptapleurum rhododendrifolium (Griff.) Lowry \& G. M. Plunkett [New Combination]

B.: Panax rhododendrifolius Griff.

LOWRY Porter P., PLUNKETT Gregory M., Resurrection of the Genus Heptapleurum for the Asian Clade of Species Previously Included in Schefflera (Araliaceae). Novon 28 (3): 143-170. 2020 [164] doi: 10.3417/2020612

CHINA (Xizang); BHUTAN, INDIA, NEPAL

Heptapleurum shweliense (W. W. Sm.) Lowry \& G. M. Plunkett [New Combination]

B.: Schefflera shweliensis W. W. Sm.

LOWRY Porter P., PLUNKETT Gregory M., Resurrection of the Genus Heptapleurum for the Asian Clade of Species Previously Included in Schefflera (Araliaceae). Novon 28 (3): 143-170. 2020 [166] doi: 10.3417/2020612

CHINA (Yunnan); INDIA

Heptapleurum wardii (C. Marquand \& Airy Shaw) Lowry \& G. M. Plunkett [New Combination]

B.: Schefflera wardii Marquand \& Airy Shaw

LOWRY Porter P., PLUNKETT Gregory M., Resurrection of the Genus Heptapleurum for the Asian Clade of Species Previously Included in Schefflera (Araliaceae). Novon 28 (3): 143-170. 2020 [168] doi: 10.3417/2020612

CHINA (Xizang, Yunnan); MYANMAR

Heptapleurum zhuanum (Lowry \& C. B. Shang) Lowry \& G. M. Plunkett [New Combination]

B.: Schefflera zhuana Lowry \& C. B. Shang

LOWRY Porter P., PLUNKETT Gregory M., Resurrection of the Genus Heptapleurum for the Asian Clade of Species Previously Included in Schefflera (Araliaceae). Novon 28 (3): 143-170. 2020 [168] doi: 10.3417/2020612

CHINA (Xizang)

\section{Arecaceae 棕㭣科}

Calamus nambariensis Becc. subsp. arunachalensis Deka, Borthakur \& Tanti [New Subspecies]

DEKA Kishor, BORTHAKUR Sashin Kumar, TANTI Bhaben, Taxonomic Distinction of Calamus nambariensis Becc., in Northeast India Based on Morphological and Molecular Markers. Taiwania 65 (4): 529-540. 2020 [536] doi: 10.6165/tai.2020.65.529

CHINA (Xizang*); INDIA

\section{Aristolochiaceae 马晚铃科}

Aristolochia wenshanensis Lei Cai, D. M. He \& Z. L. Dao 文山马淣铃 [New Species] IUCN: Endangered (NE); Evidence: Morphology

CAI Lei, HE De-ming, HUANG Yu-song, DAO Zhi-ling, Aristolochia wenshanensis, a New Species of Aristolochiaceae from Karst Region in Southeastern Yunnan, China. Taiwania 65 (1): 41-46. 2020 [41] doi: 10.6165/tai.2020.65.41

CHINA (Yunnan*) 
杜诚, 刘军, 叶文, 廖帅, 葛斌杰, 刘冰, 马金双 (2021) 中国植物新分类群、新名称 2020 年度报告. 生物多样性, 29, 10111020. http://www.biodiversity-science.net/CN/10.17520/biods.2021122

Aristolochia yachangensis B. G. Huang, Yan Liu \& Y. S. Huang [New Species] IUCN: VU: D2; Evidence: Morphology

LUO Ya-jin, NI Shi-dong, JIANG Qiang, HUANG Bo-gao, LIU Yan, HUANG Yu-song, Aristolochia yachangensis, a New Species of Aristolochiaceae from Limestone Areas in Guangxi, China. PhytoKeys 153: 49-61. 2020 [41] doi: 10.3897/phytokeys.153.52796 CHINA (Guangxi*)

Isotrema brevilimbum X. X. Zhu, Jun Wang tris \& F. Cao 短檐关木通 [New Species] IUCN: DD; Evidence: Morphology

WANG Jun, YA Ji-dong, LIU Cheng, LIU Guang, CAO Feng, MA Jin-shuang, ZHU Xin-xin, Taxonomic Studies on the Genus Isotrema (Aristolochiaceae) from China: II. I. brevilimbum (Aristolochiaceae), a New Species from Guizhou, China. PhytoKeys 152: 15-25. 2020 [16] doi: 10.3897/phytokeys.152.51760

CHINA (Guizhou*)

Isotrema hei Lei Cai \& X. X. Zhu [New Species] IUCN: NE; Evidence: Morphology

CAI Lei, DAO, Zhi-ling, ZHU Xin-xin, Isotrema hei (Aristolochiaceae), a New Species from Yunnan, China. Annales Botanici Fennici 57 (1-3): 125-129. 2020 [125] doi: 10.5735/085.057.0117

CHINA (Yunnan*)

Isotrema kechangense (Y. D. Peng \& L. Y. Yu) X. X. Zhu, Jun Wang tris \& J. S. Ma [New Combination]

B.: Aristolochia kechangensis Y. D. Peng \& L. Y. Yu

WANG Jun, MA Jin-shuang, ZHU Xin-xin, Four New Combinations in Isotrema (Aristolochiaceae). Phytotaxa 437 (3): 174-176. 2020

[174] doi: 10.11646/phytotaxa.437.3.8

CHINA (Guangxi*)

Isotrema pseudoutriforme (X. X. Zhu \& J. S. Ma) X. X. Zhu, Jun Wang tris \& J. S. Ma [New Combination]

B.: Aristolochia pseudoutriformis X. X. Zhu \& J. S. Ma

WANG Jun, MA Jin-shuang, ZHU Xin-xin, Four New Combinations in Isotrema (Aristolochiaceae). Phytotaxa 437 (3): 174-176. 2020

[174] doi: 10.11646/phytotaxa.437.3.8

CHINA (Yunnan*)

Isotrema wenshanense (Lei Cai, D. M. He \& Z. L. Dao) X. X. Zhu, Jun Wang tris \& J. S. Ma [New Combination]

B.: Aristolochia wenshanensis Lei Cai, D. M. He \& Z. L. Dao

WANG Jun, MA Jin-shuang, ZHU Xin-xin, Four New Combinations in Isotrema (Aristolochiaceae). Phytotaxa 437 (3): 174-176. 2020

[174] doi: 10.11646/phytotaxa.437.3.8

CHINA (Yunnan*)

Isotrema yangii (X. X. Zhu \& J. S. Ma) X. X. Zhu, Jun Wang tris \& J. S. Ma [New Combination]

B.: Aristolochia yangii X. X. Zhu \& J. S. Ma

WANG Jun, MA Jin-shuang, ZHU Xin-xin, Four New Combinations in Isotrema (Aristolochiaceae). Phytotaxa 437 (3): 174-176. 2020

[174] doi: 10.11646/phytotaxa.437.3.8

CHINA (Yunnan*)

\section{Asparagaceae 天门冬科}

Aspidistra brachypetala C. R. Lin \& B. Pan 短瓣蜘蛛抱蛋 [New Species] IUCN: NE; Evidence: Morphology

PAN Bo, YUAN Quan, LIN Chun-rui, Aspidistra brachypetala (Asparagaceae), a New Species from Guangxi, China. Taiwania 65 (2): 195-198. 2020 [195] doi: 10.6165/tai.2020.65.195

CHINA (Guangxi*)

Aspidistra dahuaensis D. X. Nong \& L. Y. Yu [New Species] IUCN: NE; Evidence: Morphology

NONG Dong-xin, PENG Yu-de, KE Fang, YU Li-ying, Aspidistra dahuaensis (Asparagaceae), a New Species from Guangxi, China. Phytotaxa 472 (1): 84-86. 2020 [84] doi: 10.11646/phytotaxa.472.1.12

CHINA (Guangxi*)

Aspidistra jiangjinensis S. R. Yi \& C. R. Lin [New Species] IUCN: NE; Evidence: Morphology

YI Si-yong, HUANG Ya, CHEN Hong-yuan, ZHOU Xiao-xu, LIN Chun-rui, Aspidistra jiangjinensis (Asparagaceae), a New Species from Chongqing, China. Phytotaxa 437 (3): 164-168. 2020 [164] doi: 10.11646/phytotaxa.437.3.6

CHINA (Chongqing*)

Aspidistra longiconnectiva C. T. Lu, K. C. Chuang \& J. C. Wang 长药隔蜘蛛抱蛋 [New Species] IUCN: EN: B1b(iii)B2b(iii) +C1; Evidence: Morphology

LU Chang-tse, CHUANG Kuei-chun, TSENG Yen-hsueh, WANG Chao-chien Jenn-Che WANG Jenn-che, Taxonomic Revision of Aspidistra Ker-Gawl. (Asparagaceae) in Taiwan. Taiwania 65 (3): 277-285. 2020 [281] doi: 10.6165/tai.2020.65.277

CHINA (Taiwan*)

Aspidistra xichouensis Lei Cai, Z. L. Dao \& G. W. Hu 西畴蜘蛛抱蛋 [New Species] IUCN: NE; Evidence: Morphology 
杜诚, 刘军, 叶文, 廖帅, 葛斌杰, 刘冰, 马金双 (2021) 中国植物新分类群、新名称 2020 年度报告. 生物多样性, 29, 10111020. http://www.biodiversity-science.net/CN/10.17520/biods.2021122

CAI Lei, PAN Bo, LIN Chun-rui, DAO Zhi-ling, HU Guang-wan, Aspidistra xichouensis (Asparagaceae), a New Species from the Karst Region in Southeastern Yunnan, China. Phytotaxa 439 (2): 143-149. 2020 [145] doi: 10.11646/phytotaxa.439.2.5

CHINA (Yunnan*)

Peliosanthes ligniradicis N. Tanaka, M.T aram \& D. Borah [New Species] IUCN: NE; Evidence: Morphology

TARAM Momang, BORAH Dipankar, TANAKA Noriyuki, Peliosanthes ligniradicis, a New Species (Asparagaceae) from Arunachal Pradesh, NE India. Phytotaxa 438 (1): 43-48. 2020 [44] doi: 10.11646/phytotaxa.438.1.5

CHINA (Xizang*)

Polygonatum daminense H. J. Yang \& D. F. Cui 大皿黄精 [New Species] IUCN: DD; Evidence: rbcL, matK, psbA-trnH, trnC-petN; Morphology

YANG Hai-jun, WU Bao-huan, CHEN Zi-lin, WANG Pan, SHI Shi, LI Fei-fei, XU Han, CUI Da-fang, Polygonatum daminense (Asparagaceae), a New Species from China Based on Morphological and Molecular Evidence. Phytotaxa 449 (3): 287-294. 2020 [290] doi: 10.11646/phytotaxa.449.3.7

CHINA (Zhejiang*)

Tupistra cardinalis Aver., N.Tanaka \& Son 红柱开口箭 [New Recorded]

NONG Dong-xin, PENG Yu-de, YU Li-ying, XU Wei-bin, Notes on Tupistra (Asparagaceae) in Guangxi. Guihaia 40 (7): $921-925$. 2020 [922] doi: 10.11931/guihaia.gxzw201903048

CHINA (Guangxi); VIETNAM*

Tupistra nganii K. S. Nguyen, Aver., N. Tanaka \& Nuraliev [New Species] IUCN: DD; Evidence: Morphology

AVERYANOV Leonid V., NGUYEN Khang Sinh, NURALIEV Maxim S., VISLOBOKOV Nikolay A., TANAKA Noriyuki, KOPYLOV-GUSKOV Yury O., LYSKOV Dmitry F., MAISAK Tatiana V., HIEU Nguyen Quang, KUZNETSOV Andrey N., KUZNETSOVA Svetlana P., THAI Tran Huy, Tupistra nganii (Asparagaceae), a New Species with Greenish Yellow Flowers from Northern Vietnam and Southwestern China. Phytotaxa 449 (2): 173-180. 2020 [174] doi: 10.11646/phytotaxa.449.2.5

CHINA (Yunnan); VIETNAM*

Tupistra stoliczkana Kurz [New Synonyms]

S.: Tupistra ashihoi D. K. Roy, N. Odyuo \& Aver.

BORAH Dipankar, TANAKA Noriyuki, AVERYANOV Leonid V., TARAM Momang, ROY Dilip Kumar, Rediscovery of Tupistra stoliczkana (Asparagaceae) in Northeastern India and the Identity of T. ashihoi. Phytotaxa 443 (2): 207-210. 2020 [208] doi: 10.11646/phytotaxa.443.2.8

CHINA (Xizang); INDIA, MYANMAR*

\section{Asphodelaceae 阿福花科}

Hemerocallis lilioasphodelus L. var. minor (Mill.) M. N. Tamura [New Combination]

B.: Hemerocallis minor Mill.

MURAKAMI Sho, TAKAYAMA Koji, FUSE Shizuka, HIROTA Shun K., KOI Satosh, IDENO Takahito, YAMAMOTO Takenori, TAMURA Minoru N., Recircumscription of Sections of Hemerocallis (Asphodelaceae) from Japan and Adjacent Regions Based on MIG-seq Data. Acta Phytotaxonomica \& Geobotanica 71 (1): 1-11. 2020 [8] doi: 10.18942/apg.201913

CHINA (Gansu, Hebei, Heilongjiang, Jilin, Liaoning, Nei Mongol, Shaanxi, Shandong, Shanxi); KOREA, JAPAN, MONGOLIA, RUSSIA

\section{Aspleniaceae 铁角蕨科}

Asplenium normale D. Don f. scythiforme Z. X. Chang 镰羽铁角蕨 [New Form]

CHANG Zhi-xiang, KUO Li-yaung, LU Pi-fong, HUANG Yao-moan, New Addition to the Asplenium normale complex (Aspleniaceae): an Endemic Forma in Taiwan. Taiwania 65 (2): 253-260. 2020 [253] doi: 10.6165/tai.2020.65.253 CHINA (Taiwan*)

\section{Asteraceae 菊科}

Acmella brachyglossa Cass. 短舌花金钮扣 [New Record]

HE Chun-mei, TONG Yi-hua, XIA Jing, XIA Nian-he, Two Newly Naturalized Species of Asteraceae in Mainland China. Journal of Tropical and Subtropical Botany 28 (5): 523-526. 2020 [524] doi: 10.11926/jtsb.4254

CHINA (Guangdong, Taiwan); [AMERICA]

Ainsliaea daheishanensis Y. L. Peng, C. X. Yang \& Y. Luo [New Species] IUCN: NE; Evidence: Morphology

PENG Yu-lan, YANG Chen-xuan, LUO Yan, Ainsliaea daheishanensis (Asteraceae): A New Species from China. PhytoKeys 138: 233239. 2020 [234] doi: 10.3897/phytokeys.138.38566

CHINA (Yunnan*) 
杜诚, 刘军, 叶文, 廖帅, 葛斌杰, 刘冰, 马金双 (2021) 中国植物新分类群、新名称 2020 年度报告. 生物多样性, 29, 10111020. http://www.biodiversity-science.net/CN/10.17520/biods.2021122

Ainsliaea wuyuanensis Z. H. Chen, Y. L. Xu \& X. F. Jin 敂源兔儿风 [New Species] IUCN: NE; Evidence: Morphology

XU Yue-liang, CHEN Feng, HONG Yuan-hua, CHEN Zheng-hai, JIN Xiao-feng, A New Species of Ainsliaea DC. (Asteraceae) from Northeast Jiangxi. Guihaia 40 (1): 95-98. 2020 [96] doi: 10.11931/guihaia.gxzw201907064

CHINA (Jiangxi*)

Artemisia rutifolia Steph. ex Spreng. var. ruoqiangensis Y. R. Ling [Typification]

GUO Xin-qiang, WANG Long, YANG Qin-er, Lectotypification of Artemisia rutifolia var. ruoqiangensis (Asteraceae, Anthemideae). Phytotaxa 453 (1): 49-51. 2020 [49] doi: 10.11646/phytotaxa.453.1.5

CHINA (Xinjiang*)

Artemisia sichuanensis Y. Ling \& Y. R. Ling var. tomentosa Y. R. Ling [New Synonyms]

S.: Artemisia erlangshanensis Y. Ling \& Y. R. Ling

GUO Xin-qiang, WANG Long, YANG Qin-er, Clarification of Morphological Distinction between Artemisia sichuanensis var. sichuanensis (Asteraceae, Anthemideae) and A. sichuanensis var. tomentosa, with Reduction of A. erlangshanensis to the Synonymy of the Latter Variety. Phytotaxa 453 (1): 16-30. 2020 [26] doi: 10.11646/phytotaxa.453.1.2

CHINA (Sichuan*)

Artemisia waltonii J. R. Drumm. ex Pamp. [Typification]

GUO Xin-qiang, WANG Long, YANG Qin-er, Taxonomic Notes on Artemisia waltonii (Asteraceae, Anthemideae), with Reduction of A. kangmarensis and A. conaensis to the Synonymy of Its Type Variety. Phytotaxa 450 (2): 149-172. 2020 [161] doi: 10.11646/phytotaxa.450.2.2

CHINA (Xizang*)

Artemisia waltonii J. R. Drumm. ex Pamp. var. waltonii [New Synonyms]

S.: Artemisia kangmarensis Y. Ling \& Y. R. Ling; Artemisia conaensis Y. Ling \& Y. R. Ling

GUO Xin-qiang, WANG Long, YANG Qin-er, Taxonomic Notes on Artemisia waltonii (Asteraceae, Anthemideae), with Reduction of A. kangmarensis and A. conaensis to the Synonymy of Its Type Variety. Phytotaxa 450 (2): 149-172. 2020 [169] doi: 10.11646/phytotaxa.450.2.2

CHINA (Xizang*)

Aster bodinieri H. Lév. [Typification]

NESOM Guy L., Tibetiodes Nesom, gen. nov. (Asteraceae: Astereae), including 27 Species of Himalayan Aster. Phytoneuron (63): 130. 2020 [3]

CHINA (Yunnan*)

Aster chuanshanensis W. P. Li [New Species] IUCN: NE; Evidence: ITS, ETS; Chromosome; Morphology

XIAO Jia-wei, ZHAO Qing-ya, XIONG Yi-chang, LI Wei-ping, Aster chuanshanensis (Asteraceae), a New Species from Shanxi and Sichuan, China. Annales Botanici Fennici 57 (4-6): 341-350. 2020 [342] doi: 10.5735/085.057.0417

CHINA (Shaanxi*, Sichuan)

Aster delavayi Franch. [Typification]

NESOM Guy L., Tibetiodes Nesom, gen. nov. (Asteraceae: Astereae), including 27 Species of Himalayan Aster. Phytoneuron (63): 130. $2020[3]$

CHINA (Yunnan*)

Aster flaccidus Bunge var. fructu-glandulosus Ostenf. [Typification]

NESOM Guy L., Tibetiodes Nesom, gen. nov. (Asteraceae: Astereae), including 27 Species of Himalayan Aster. Phytoneuron (63): 130. $2020[4]$

CHINA (Xizang*)

Aster fordii Hemsl. [Typification]

NESOM Guy L., Metamyriactis (Asteraceae, Astereae), a New Genus of Southeast Asian Asters. Phytoneuron (58): 1-36. 2020 [3]

CHINA (Guangdong*)

Aster huangpingensis W. P. Li \& Z. Li 黄平紫苑 [New Species] IUCN: EN: D1; Evidence: ITS, ETS; Chromosome; Morphology

LI Zhi, XIONG Yi-chang, LIAO Jun-jie, XIAO Jia-wei, LI Wei-ping, Aster huangpingensis (Asteraceae, Astereae), a New Species from Guizhou, China. Phytotaxa 433 (3): 235-244. 2020 [242] doi: 10.11646/phytotaxa.433.3.5

CHINA (Guizhou*)

Aster kanoi S. W. Chung, W. J. Huang \& T. C. Hsu 鹿野氏马兰 [New Species] IUCN: NT: B2b(iii); C2a(i); D; Evidence: Morphology CHUNG Shih-wen, HUANG Wei-jie, HSU Tian-chuan, Aster kanoi (Asteraceae, Astereae), a New Species from Taiwan, with Lectotypification and Morphological Emendation of Aster takasagomontanus. Taiwania 65 (3): 315-320. 2020 [316] doi: 10.6165/tai.2020.65.315 
杜诚, 刘军, 叶文, 廖帅, 葛斌杰, 刘冰, 马金双 (2021) 中国植物新分类群、新名称 2020 年度报告. 生物多样性, 29, 10111020. http://www.biodiversity-science.net/CN/10.17520/biods.2021122

CHINA (Taiwan*)

Aster mekongensis Onno [Typification]

NESOM Guy L., Tibetiodes Nesom, gen. nov. (Asteraceae: Astereae), including 27 Species of Himalayan Aster. Phytoneuron (63): 130. $2020[5]$

CHINA (Yunnan*)

Aster nigrotinctus Y. Ling [Typification]

NESOM Guy L., Tibetiodes Nesom, gen. nov. (Asteraceae: Astereae), including 27 Species of Himalayan Aster. Phytoneuron (63): 130. $2020[4]$

CHINA (Xizang*)

Aster staticifolius Franch. [Typification]

NESOM Guy L., New Genera from Asian Aster (Asteraceae: Astereae). Phytoneuron (64): 1-44. 2020 [1]

CHINA (Yunnan*)

Aster takasagomontanus Sasaki 雪山马兰 [Typification]

CHUNG Shih-wen, HUANG Wei-jie, HSU Tian-chuan, Aster kanoi (Asteraceae, Astereae), a New Species from Taiwan, with Lectotypification and Morphological Emendation of Aster takasagomontanus. Taiwania 65 (3): 315-320. 2020 [319] doi: 10.6165/tai.2020.65.315

CHINA (Taiwan*)

Aster vilmorinii Franch. [Typification]

NESOM Guy L., Tibetiodes Nesom, gen. nov. (Asteraceae: Astereae), including 27 Species of Himalayan Aster. Phytoneuron (63): 130. 2020 [7]

CHINA (Sichuan*)

Blumea hunanensis L. D. Duan \& Yun Lin [New Species] IUCN: NE; Evidence: Morphology

DUAN Lin-dong, LIN Yun, YANG Zhi-rong, LU Zhen, Blumea hunanensis (Asteraceae: Inuleae), a New Species from Hunan, China. Kew Bulletin 75 (9): 1-8. 2020 [1] doi: 10.1007/S12225-020-9860-Z

CHINA (Hunan*)

Cardiagyris G. L. Nesom [New Genus]

NESOM Guy L., Cardiagyris (Asteraceae: Astereae), a New Genus for the Doelllingeria-like Species of Asia. Phytoneuron (61): 1-26. 2020 [1]

CHINA

Cardiagyris dolichophylla (Y. Ling) G. L. Nesom [New Combination]

B.: Aster dolichophyllus Y. Ling

NESOM Guy L., Cardiagyris (Asteraceae: Astereae), a New Genus for the Doelllingeria-like Species of Asia. Phytoneuron (61): 1-26. 2020 [5]

CHINA (Guangxi*)

Cardiagyris dolichophylla (Y. Ling) G. L. Nesom [New Combination]

B.: Aster huangpingensis W. P. Li \& Zhi Li

NESOM Guy L., Cardiagyris (Asteraceae: Astereae), a New Genus for the Doelllingeria-like Species of Asia. Phytoneuron (61): 1-26. 2020 [5]

CHINA (Guizhou*)

Cardiagyris marchandii (H. Lév.) G. L. Nesom [New Combination]

B.: Aster marchandii H. Lév.

NESOM Guy L., Cardiagyris (Asteraceae: Astereae), a New Genus for the Doelllingeria-like Species of Asia. Phytoneuron (61): 1-26. 2020 [3]

CHINA (Fujian, Guangdong, Guangxi, Guizhou*, Hubei, Jiangxi, Sichuan, Zhejiang)

Cardiagyris scabra (Thunb.) G. L. Nesom [New Combination]

B.: Aster scaber Thunb.

NESOM Guy L., Cardiagyris (Asteraceae: Astereae), a New Genus for the Doelllingeria-like Species of Asia. Phytoneuron (61): 1-26. 2020 [3]

CHINA (Anhui, Fujian, Guangdong, Guangxi, Guizhou, Hebei, Heilongjiang, Henan, Hubei, Hunan, Jiangsu, Jiangxi, Jilin, Liaoning, Nei Mongol, Shaanxi, Shandong, Shanxi, Sichuan, Zhejiang); JAPAN*, KOREA, RUSSIA

Chaochienchangia G. L. Nesom [New Genus]

NESOM Guy L., Yonglingia, Chaochienchangia, and Sinobouffordia (Asteraceae: Astereae), New Genera Segregated from Aster in China. Phytoneuron (57): 1-25. 2020 [9]

CHINA 
杜诚, 刘军, 叶文, 廖帅, 葛斌杰, 刘冰, 马金双 (2021) 中国植物新分类群、新名称 2020 年度报告. 生物多样性, 29, 10111020. http://www.biodiversity-science.net/CN/10.17520/biods.2021122

Chaochienchangia falcifolia (Hand.-Mazz.) G. L. Nesom [New Combination]

B.: Aster falcifolius Hand.-Mazz.

NESOM Guy L., Yonglingia, Chaochienchangia, and Sinobouffordia (Asteraceae: Astereae), New Genera Segregated from Aster in

China. Phytoneuron (57): 1-25. 2020 [9]

CHINA (Gansu, Hubei*, Shaanxi, Sichuan)

Chlamydites prainii J. R. Drumm. [Typification]

NESOM Guy L., Chlamydites (Asteraceae: Astereae) Revived. Phytoneuron (59): 1-14. 2020 [2]

CHINA (Sichuan, Xizang*)

Chrysanthemum bizarre C. Z. Shen [New Species] IUCN: NE; Evidence: Morphology

MENG Shi-yong, WU Lei, SHEN Chu-ze, Chrysanthemum bizarre, a New Species of Chrysanthemum from Hunan, China. Phytotaxa 442 (3): 215-224. 2020 [220] doi: 10.11646/phytotaxa.442.3.7

CHINA (Hunan*)

Chrysanthemum bizarre C. Z. Shen [Typification]

MENG Shi-yong, WU Lei, SHEN Chu-ze, Chrysanthemum bizarre, a New Species of Chrysanthemum from Hunan, China. Phytotaxa 442 (3): 215-224. 2020 [220] doi: 10.11646/phytotaxa.442.3.7

CHINA (Hubei*)

Cordiofontis G. L. Nesom [New Genus]

NESOM Guy L., Cordiofontis (Asteraceae: Astereae), a New Genus of Himalayan Species Segregated from Aster. Phytoneuron (60): 1-20. 2020 [1]

CHINA

Cordiofontis longipetiolata (C. C. Chang) G. L. Nesom [New Combination]

B.: Aster longipetiolatus C. C. Chang

NESOM Guy L., Cordiofontis (Asteraceae: Astereae), a New Genus of Himalayan Species Segregated from Aster. Phytoneuron (60): 1-20. 2020 [3]

CHINA (Sichuan*, Yunnan)

Emilia praetermissa Milne-Redh. 粉黄姃线花 [New Record]

HE Chun-mei, TONG Yi-hua, XIA Jing, XIA Nian-he, Two Newly Naturalized Species of Asteraceae in Mainland China. Journal of Tropical and Subtropical Botany 28 (5): 523-526. 2020 [524] doi: 10.11926/jtsb.4254

CHINA (Guangdong, Taiwan); [AFRICA]

Erigeron panduratus C. C. Chang [Typification]

NESOM Guy L., Metamyriactis (Asteraceae, Astereae), a New Genus of Southeast Asian Asters. Phytoneuron (58): 1-36. 2020 [3]

CHINA (Guangxi*)

Gelasia circumflexa (Krasch. \& Lipsch.) Zaika, Sukhor. \& N. Kilian [New Combination]

B.: Scorzonera circumflexa Krasch. \& Lipsch.

ZAIKA Maxim A., KILIAN Norbert, JONES Katy, KRINITSINA Anastasiya A., NILOVA Maya V., SPERANSKAYA Anna S., SUKHORUKOV Alexander P., Scorzonera sensu lato (Asteraceae, Cichorieae) - Taxonomic Reassessment in the Light of New Molecular Phylogenetic and Carpological Analyses. PhytoKeys 137: 1-85. 2020 [57] doi: 10.3897/phytokeys.137.46544

CHINA (Xinjiang); AFGHANISTAN, UZBEKISTAN

Gelasia ensifolia (M. Bieb.) Zaika, Sukhor. \& N. Kilian [New Combination]

B.: Scorzonera ensifolia M. Bieb.

ZAIKA Maxim A., KILIAN Norbert, JONES Katy, KRINITSINA Anastasiya A., NILOVA Maya V., SPERANSKAYA Anna S., SUKHORUKOV Alexander P., Scorzonera sensu lato (Asteraceae, Cichorieae) - Taxonomic Reassessment in the Light of New Molecular Phylogenetic and Carpological Analyses. PhytoKeys 137: 1-85. 2020 [57] doi: 10.3897/phytokeys.137.46544

CHINA (Xinjiang); KAZAKHSTAN, RUSSIA

Geothamnus G. L. Nesom [New Genus]

NESOM Guy L., New Genera from Asian Aster (Asteraceae: Astereae). Phytoneuron (64): 1-44. 2020 [1]

CHINA

Geothamnus batangensis (Bureau \& Franch.) G. L. Nesom [New Combination]

B.: Aster batangensis Bureau \& Franch.

NESOM Guy L., New Genera from Asian Aster (Asteraceae: Astereae). Phytoneuron (64): 1-44. 2020 [1]

CHINA (Sichuan*)

Griersonia G. L. Nesom [New Genus] 
杜诚, 刘军, 叶文, 廖帅, 葛斌杰, 刘冰, 马金双 (2021) 中国植物新分类群、新名称 2020 年度报告. 生物多样性, 29, 10111020. http://www.biodiversity-science.net/CN/10.17520/biods.2021122

NESOM Guy L., New Genera from Asian Aster (Asteraceae: Astereae). Phytoneuron (64): 1-44. 2020 [31] CHINA

Griersonia fuscescens (Bureau \& Franch.) G. L. Nesom [New Combination]

B.: Aster fuscescens Bureau \& Franch.

NESOM Guy L., New Genera from Asian Aster (Asteraceae: Astereae). Phytoneuron (64): 1-44. 2020 [31]

CHINA (Sichuan*, Yunnan)

Griersonia oblongifolia (Grierson) G. L. Nesom [New Combination]

B.: Aster fuscescens Bureau \& Franch. var. oblongifolius Grierson

NESOM Guy L., New Genera from Asian Aster (Asteraceae: Astereae). Phytoneuron (64): 1-44. 2020 [32]

CHINA (Sichuan*, Yunnan)

Griersonia senecioides (Franch.) G. L. Nesom [New Combination]

B.: Aster senecioides Franch.

NESOM Guy L., New Genera from Asian Aster (Asteraceae: Astereae). Phytoneuron (64): 1-44. 2020 [32]

CHINA (Yunnan*)

Iteroloba G. L. Nesom [New Genus]

NESOM Guy L., New Genera from Asian Aster (Asteraceae: Astereae). Phytoneuron (64): 1-44. 2020 [7]

CHINA

Iteroloba bipinnatisecta (Ludlow ex Grierson ) G. L. Nesom [New Combination]

B.: Aster bipinnatisectus Ludlow ex Grierson

NESOM Guy L., New Genera from Asian Aster (Asteraceae: Astereae). Phytoneuron (64): 1-44. 2020 [7]

CHINA (Xizang*)

Jurinea auriculata (DC.) N. Garcia, Herrando \& Susanna [New Combination]

B.: Aplotaxis auriculata DC.

HERRANDO-MORAIRA Sonia, CALLEJA Juan-Antonio, CHEN You-sheng, FUJIKAWA Kazumi, GALBANY-CASALS Mercè, GARCIA-JACAS Núria, KIM Seung-Chul, LIU Jian-quan, LÓPEZ-ALVARADO Javier, LÓPEZ-PUJOL Jordi, MANDEL Jennifer R., MEHREGAN Iraj, ROQUET Cristina, SENNIKOV, Generic Boundaries in subtribe Saussureinae (Compositae: Cardueae): Insights from Hyb-Seq Data. Taxon 69 (4): 694-714. 2020 [708] doi: 10.1002/tax.12314

CHINA (Xizang); BHUTAN, INDIA, NEPAL

Jurinea deltoidea (DC.) N. Garcia, Herrando \& Susanna [New Combination]

B.: Aplotaxis deltoidea DC.

HERRANDO-MORAIRA Sonia, CALLEJA Juan-Antonio, CHEN You-sheng, FUJIKAWA Kazumi, GALBANY-CASALS Mercè, GARCIA-JACAS Núria, KIM Seung-Chul, LIU Jian-quan, LÓPEZ-ALVARADO Javier, LÓPEZ-PUJOL Jordi, MANDEL Jennifer R., MEHREGAN Iraj, ROQUET Cristina, SENNIKOV, Generic Boundaries in subtribe Saussureinae (Compositae: Cardueae): Insights from Hyb-Seq Data. Taxon 69 (4): 694-714. 2020 [708] doi: 10.1002/tax.12314

CHINA (Anhui, Fujian, Guangdong, Guangxi, Guizhou, Henan, Hubei, Hunan, Jiangxi, Shaanxi, Sichuan, Taiwan, Xizang, Yunnan, Zhejiang); BHUTAN, INDIA, LAOS, MYANMAR, NEPAL, PAKISTAN, THAILAND, VIETNAM

Jurinea peguensis (C. B. Clarke) N. Garcia, Herrando \& Susanna [New Combination]

B.: Saussurea peguensis C. B. Clarke

HERRANDO-MORAIRA Sonia, CALLEJA Juan-Antonio, CHEN You-sheng, FUJIKAWA Kazumi, GALBANY-CASALS Mercè, GARCIA-JACAS Núria, KIM Seung-Chul, LIU Jian-quan, LÓPEZ-ALVARADO Javier, LÓPEZ-PUJOL Jordi, MANDEL Jennifer R., MEHREGAN Iraj, ROQUET Cristina, SENNIKOV, Generic Boundaries in subtribe Saussureinae (Compositae: Cardueae): Insights from Hyb-Seq Data. Taxon 69 (4): 694-714. 2020 [709] doi: 10.1002/tax.12314

CHINA (Guizhou, Yunnan); MYANMAR, THAILAND

Jurinea yakla (C. B. Clarke) N. Garcia, Herrando \& Susanna [New Combination]

B.: Saussurea yakla C. B. Clarke

HERRANDO-MORAIRA Sonia, CALLEJA Juan-Antonio, CHEN You-sheng, FUJIKAWA Kazumi, GALBANY-CASALS Mercè, GARCIA-JACAS Núria, KIM Seung-Chul, LIU Jian-quan, LÓPEZ-ALVARADO Javier, LÓPEZ-PUJOL Jordi, MANDEL Jennifer R., MEHREGAN Iraj, ROQUET Cristina, SENNIKOV, Generic Boundaries in subtribe Saussureinae (Compositae: Cardueae): Insights from Hyb-Seq Data. Taxon 69 (4): 694-714. 2020 [709] doi: 10.1002/tax.12314

CHINA (Xizang); BHUTAN, INDIA, NEPAL

Lactuca hirsuta Franch. [Typification]

WANG Ze-huan, KILIAN Norbert, CHEN Ya-ping, PENG Hua, Sinoseris (Crepidinae, Cichorieae, Asteraceae), a New Genus of Three Species Endemic to China, One of Them New to Science. Willdenowia 50 (1): 91-110. 2020 [103] doi: 10.3372/wi.50.50109

CHINA (Yunnan*) 
杜诚, 刘军, 叶文, 廖帅, 葛斌杰, 刘冰, 马金双 (2021) 中国植物新分类群、新名称 2020 年度报告. 生物多样性, 29, 10111020. http://www.biodiversity-science.net/CN/10.17520/biods.2021122

Lipschitzia Zaika, Sukhor. \& N. Kilian [New Genus]

ZAIKA Maxim A., KILIAN Norbert, JONES Katy, KRINITSINA Anastasiya A., NILOVA Maya V., SPERANSKAYA Anna S., SUKHORUKOV Alexander P., Scorzonera sensu lato (Asteraceae, Cichorieae) - Taxonomic Reassessment in the Light of New Molecular Phylogenetic and Carpological Analyses. PhytoKeys 137: 1-85. 2020 [61] doi: 10.3897/phytokeys.137.46544 CHINA

Lipschitzia divaricata (Turcz.) Zaika, Sukhor. \& N. Kilian [New Combination]

B.: Scorzonera divaricata Turcz.

ZAIKA Maxim A., KILIAN Norbert, JONES Katy, KRINITSINA Anastasiya A., NILOVA Maya V., SPERANSKAYA Anna S., SUKHORUKOV Alexander P., Scorzonera sensu lato (Asteraceae, Cichorieae) - Taxonomic Reassessment in the Light of New Molecular Phylogenetic and Carpological Analyses. PhytoKeys 137: 1-85. 2020 [62] doi: 10.3897/phytokeys.137.46544 CHINA (Hebei, Shaanxi, Shanxi, Nei Mongol); MONGOLIA

Metamyriactis nigromontana (Dunn) G. L. Nesom [New Combination]

B.: Aster nigromontanus Dunn

NESOM Guy L., Metamyriactis (Asteraceae, Astereae), a New Genus of Southeast Asian Asters. Phytoneuron (58): 1-36. 2020 [4]

CHINA (Yunnan*)

Metamyriactis pandurata (Nees ex Walp.) G. L. Nesom [New Combination]

B.: Aster pandurata Nees ex Walp.

NESOM Guy L., Metamyriactis (Asteraceae, Astereae), a New Genus of Southeast Asian Asters. Phytoneuron (58): 1-36. 2020 [3]

CHINA (Fujian, Gansu, Guangdong*, Guangxi, Guizhou, Hong Kong, Hubei, Henan, Jiangsu, Jiangxi, Sichuan, Xizang, Yunnan, Zhejiang); THAILAND

Metamyriactis pycnophylla (Franch. ex Diels) G. L. Nesom [New Combination]

B.: Aster pycnophyllus Franch. ex Diels

NESOM Guy L., Metamyriactis (Asteraceae, Astereae), a New Genus of Southeast Asian Asters. Phytoneuron (58): 1-36. 2020 [3]

CHINA (Yunnan*)

Metamyriactis sikkimensis (Hook. f.) G. L. Nesom [New Combination]

B.: Aster sikkimensis Hook. f.

NESOM Guy L., Metamyriactis (Asteraceae, Astereae), a New Genus of Southeast Asian Asters. Phytoneuron (58): 1-36. 2020 [4]

CHINA (Xizang); INDIA*, NEPAL

Metamyriactis sikkimensis (Hook. f.) G. L. Nesom [Typification]

NESOM Guy L., Metamyriactis (Asteraceae, Astereae), a New Genus of Southeast Asian Asters. Phytoneuron (58): 1-36. 2020 [4]

CHINA (Xizang); INDIA*, NEPAL

Metamyriactis pycnophylla (Hutch. \& J. R. Drumm. ex G. J. Zhang \& T. G. Gao) G. L. Nesom [New Combination]

B.: Aster veitchiana Hutch. \& J. R. Drumm. ex G. J. Zhang \& T. G. Gao

NESOM Guy L., Metamyriactis (Asteraceae, Astereae), a New Genus of Southeast Asian Asters. Phytoneuron (58): 1-36. 2020 [4]

CHINA (Sichuan*)

Pertya cordifolia Mattf. [Typification]

ZHANG Cai-fei, LIU Sheng-long, LI Liang-qian, Pertya ferruginea (Asteraceae: Pertyeae), a New Species from Southeastern China, with a Revision of P. cordifolia and P. pubescens. Phytotaxa 474 (3): 235-249. 2020 [236] doi: 10.11646/phytotaxa.474.3.3

CHINA (Guangdong, Guangxi, Hunan*)

Pertya ferruginea Cai F. Zhang 锈毛帚菊 [New Species] IUCN: VU: B2ab(ii, iii); Evidence: Morphology

ZHANG Cai-fei, LIU Sheng-long, LI Liang-qian, Pertya ferruginea (Asteraceae: Pertyeae), a New Species from Southeastern China, with a Revision of P. cordifolia and P. pubescens. Phytotaxa 474 (3): 235-249. 2020 [242] doi: 10.11646/phytotaxa.474.3.3

CHINA (Fujian, Jiangxi, Zhejiang*)

Pertya huangii Huan C. Wang \& Q. P. Wang [New Species] IUCN: NE; Evidence: Morphology

PAN Bo, XU Mei-zhen, TANG Wen-xiu, YANG Li-hua, Pertya huangii (Asteraceae), a New Species from Yunnan, Southwest China. Annales Botanici Fennici 57 (1-3): 87-91. 2020 [87] doi: 10.5735/085.057.0112

CHINA (Yunnan*)

Pseudopodospermum inconspicuum (Lipsch.) Zaika, Sukhor. \& N. Kilian [New Combination]

B.: Scorzonera inconspicua Lipsch.

ZAIKA Maxim A., KILIAN Norbert, JONES Katy, KRINITSINA Anastasiya A., NILOVA Maya V., SPERANSKAYA Anna S., SUKHORUKOV Alexander P., Scorzonera sensu lato (Asteraceae, Cichorieae) - Taxonomic Reassessment in the Light of New Molecular Phylogenetic and Carpological Analyses. PhytoKeys 137: 1-85. 2020 [69] doi: 10.3897/phytokeys.137.46544 CHINA (Xinjiang); KAZAKHSTAN, KYRGYZSTAN, TAJIKISTAN, UZBEKISTAN

Pseudopodospermum pubescens (DC.) Zaika, Sukhor. \& N. Kilian [New Combination] 
杜诚, 刘军, 叶文, 廖帅, 葛斌杰, 刘冰, 马金双 (2021) 中国植物新分类群、新名称 2020 年度报告. 生物多样性, 29, 10111020. http://www.biodiversity-science.net/CN/10.17520/biods.2021122

B.: Scorzonera pubescens DC.

ZAIKA Maxim A., KILIAN Norbert, JONES Katy, KRINITSINA Anastasiya A., NILOVA Maya V., SPERANSKAYA Anna S., SUKHORUKOV Alexander P., Scorzonera sensu lato (Asteraceae, Cichorieae) - Taxonomic Reassessment in the Light of New Molecular Phylogenetic and Carpological Analyses. PhytoKeys 137: 1-85. 2020 [69] doi: 10.3897/phytokeys.137.46544

CHINA (Xinjiang); KAZAKHSTAN, KYRGYZSTAN, RUSSIA, TAJIKISTAN

Saussurea sagittifolia Y. S. Chen \& S. R. Yi 戟叶风毛菊 [New Species] IUCN: EN: B2ab; Evidence: Morphology

XU Lian-sheng, YI Si-rong, CHEN You-sheng, Saussurea sagittifolia (Asteraceae, Cardueae), a New Species from the Bashan Mountains Region of China. Phytotaxa 472 (3): 295-298. 2020 [295] doi: 10.11646/phytotaxa.472.3.9

CHINA (Sichuan*)

Saussurea solaris Raab-Straube \& Lidén [New Species] IUCN: EN: B1a;D; Evidence: Morphology

RAAB-STRAUBE Eckhard von, LIDÉN, Magnus, Saussurea solaris (Asteraceae, Cardueae), a New Species from East Himalaya First Collected by Francis Kingdon-Ward in 1938 and rediscovered in 2013. Symbolae Botanicae Upsalienses, Acta Universitatis Upsaliensis 40: 130-132. 2020 [130]

CHINA (Xizang*)

Saussurea xinjiangensis Y. S. Chen [New Species] IUCN: DD; Evidence: Chloroplast WGS; Morphology

CHEN You-sheng, XU Lian-sheng, Saussurea xinjiangensis sp. nov. (Asteraceae, Cardueae), a New Species from Xinjiang, China. Nordic Journal of Botany 38 (e02711): 1-7. 2020 [2] doi: 10.1111/njb.02711

CHINA (Xinjiang*)

Saussurea yilingii Y. S. Chen [New Species] IUCN: CR; Evidence: Morphology

CHEN You-sheng, Saussurea yilingii (Asteraceae, Cardueae), a New Species from Sichuan, China. Phytotaxa 452 (3): $236-240.2020$

[236] doi: 10.11646/phytotaxa.452.3.7

CHINA (Sichuan*)

Saussurea yiwuensis L. Q. Zhao \& R. Xu [New Species] IUCN: NE; Evidence: Morphology

RI Xu, ZHAO Li-qing, QING Hua, Saussurea yiwuensis (Asteraceae), a New Species from Xinjiang, China. Annales Botanici Fennici 57 (1-3): 159-161. 2020 [159] doi: 10.5735/085.057.0121

CHINA (Xinjiang*)

Senecio pelleifolius King ex J. R. Drumm. [Typification]

Fei Wen-qun, Tang Ming, Wu Xing, Ren Chen, Proposal to Conserve the Name Senecio palmatisectus (Parasenecio palmatisectus) against S. pelleifolius (Asteraceae). Taxon 69 (1): 202-203. 2020 [202] doi: 10.1002/tax.12192

CHINA (Xizang*)

Sinobouffordia G. L. Nesom [New Genus]

NESOM Guy L., Yonglingia, Chaochienchangia, and Sinobouffordia (Asteraceae: Astereae), New Genera Segregated from Aster in China. Phytoneuron (57): 1-25. 2020 [15] CHINA

Sinobouffordia poliothamnus (Diels) G. L. Nesom [New Combination]

B.: Aster poliothamnus Diels

NESOM Guy L., Yonglingia, Chaochienchangia, and Sinobouffordia (Asteraceae: Astereae), New Genera Segregated from Aster in China. Phytoneuron (57): 1-25. 2020 [16]

CHINA (Gansu, Qinghai, Shaanxi, Sichuan*, Xizang)

Sinobouffordia sikuensis (W. W. Smith \& Farrer) G. L. Nesom [New Combination]

B.: Aster sikuensis W. W. Smith \& Farrer

NESOM Guy L., Yonglingia, Chaochienchangia, and Sinobouffordia (Asteraceae: Astereae), New Genera Segregated from Aster in China. Phytoneuron (57): 1-25. 2020 [16]

CHINA (Gansu*, Shaanxi, Sichuan)

Sinosenecio ovatifolius C. Y. Zou, Yan Liu \& Ying Liu [New Species] IUCN: NE; Evidence: nrITS; Chromosome; Morphology

ZOU Chun-yu, LIU Yan, LIU Ying, Sinosenecio ovatifolius (Asteraceae), a New Species from Guangxi, China. Phytotaxa 460 (2): 149-159. 2020 [155] doi: 10.11646/phytotaxa.460.2.5

CHINA (Guangxi*)

Sinoseris N. Kilian, Ze H. Wang \& H. Peng [New Genus]

WANG Ze-huan, KILIAN Norbert, CHEN Ya-ping, PENG Hua, Sinoseris (Crepidinae, Cichorieae, Asteraceae), a New Genus of Three Species Endemic to China, One of Them New to Science. Willdenowia 50 (1): 91-110. 2020 [100] doi: 10.3372/wi.50.50109 CHINA

Sinoseris changii Ze H. Wang, N. Kilian \& H. Peng [New Species] IUCN: NE; Evidence: ITS, trnL-F, psbA-trnH, matK; Morphology 
杜诚, 刘军, 叶文, 廖帅, 葛斌杰, 刘冰, 马金双 (2021) 中国植物新分类群、新名称 2020 年度报告. 生物多样性, 29, 10111020. http://www.biodiversity-science.net/CN/10.17520/biods.2021122

WANG Ze-huan, KILIAN Norbert, CHEN Ya-ping, PENG Hua, Sinoseris (Crepidinae, Cichorieae, Asteraceae), a New Genus of Three Species Endemic to China, One of Them New to Science. Willdenowia 50 (1): 91-110. 2020 [108] doi: 10.3372/wi.50.50109 CHINA (Yunnan*)

Sinoseris muliensis (Y. S. Chen, L. S. Xu \& R. Ke) Ze H. Wang, N. Kilian \& H. Peng [New Combination]

B.: Nabalus muliensis Y. S. Chen, L. S. Xu \& R. Ke

WANG Ze-huan, KILIAN Norbert, CHEN Ya-ping, PENG Hua, Sinoseris (Crepidinae, Cichorieae, Asteraceae), a New Genus of Three Species Endemic to China, One of Them New to Science. Willdenowia 50 (1): 91-110. 2020 [105] doi: 10.3372/wi.50.50109 CHINA (Sichuan*, Yunnan)

Sinoseris scandens (C. C. Chang) Ze H. Wang, N. Kilian \& H. Peng [New Combination]

B.: Lactuca scandens C. C. Chang

WANG Ze-huan, KILIAN Norbert, CHEN Ya-ping, PENG Hua, Sinoseris (Crepidinae, Cichorieae, Asteraceae), a New Genus of Three Species Endemic to China, One of Them New to Science. Willdenowia 50 (1): 91-110. 2020 [103] doi: 10.3372/wi.50.50109 CHINA (Sichuan*, Yunnan)

Sinoseris scandens (C. C. Chang) Ze H. Wang, N. Kilian \& H. Peng [Typification]

WANG Ze-huan, KILIAN Norbert, CHEN Ya-ping, PENG Hua, Sinoseris (Crepidinae, Cichorieae, Asteraceae), a New Genus of Three Species Endemic to China, One of Them New to Science. Willdenowia 50 (1): 91-110. 2020 [103] doi: 10.3372/wi.50.50109 CHINA (Sichuan*, Yunnan)

Sinosidus G. L. Nesom [New Genus]

NESOM Guy L., New Genera from Asian Aster (Asteraceae: Astereae). Phytoneuron (64): 1-44. 2020 [12] CHINA

Sinosidus albescens (DC.) G. L. Nesom [New Combination]

B.: Amphirhapis albescens DC.

NESOM Guy L., New Genera from Asian Aster (Asteraceae: Astereae). Phytoneuron (64): 1-44. 2020 [12]

CHINA (Gansu, Guizhou, Hubei, Shaanxi, Sichuan, Xizang, Yunnan); BHUTAN, INDIA, MYANMAR, NEPAL*

Sinosidus albescens (DC.) G. L. Nesom var. discolor (Y. Ling) G. L. Nesom [New Combination]

B.: Aster albescens (DC.) Wall. ex Hand.-Mazz. var. discolor Y. Ling

NESOM Guy L., New Genera from Asian Aster (Asteraceae: Astereae). Phytoneuron (64): 1-44. 2020 [13]

CHINA (Xizang*)

Sinosidus albescens (DC.) G. L. Nesom var. glabratus (Diels) G. L. Nesom [New Combination]

B.: Aster harrowianus Diels var. glabratus Diels

NESOM Guy L., New Genera from Asian Aster (Asteraceae: Astereae). Phytoneuron (64): 1-44. 2020 [13]

CHINA (Yunnan*)

Sinosidus albescens (DC.) G. L. Nesom var. glandulosus (Hand.-Mazz.) G. L. Nesom [New Combination] B.: Aster albescens (DC.) Wall. ex Hand.-Mazz. var. glandulosus Hand.-Mazz.

NESOM Guy L., New Genera from Asian Aster (Asteraceae: Astereae). Phytoneuron (64): 1-44. 2020 [13] CHINA (Xizang*)

Sinosidus albescens (DC.) G. L. Nesom var. gracilior (Hand.-Mazz.) G. L. Nesom [New Combination] B.: Aster limprichtii Diels var. gracilior Hand.-Mazz.

NESOM Guy L., New Genera from Asian Aster (Asteraceae: Astereae). Phytoneuron (64): 1-44. 2020 [13] CHINA (Sichuan*)

Sinosidus albescens (DC.) G. L. Nesom var. glabratus (Diels) G. L. Nesom [New Combination] B.: Aster limprichtii Diels

NESOM Guy L., New Genera from Asian Aster (Asteraceae: Astereae). Phytoneuron (64): 1-44. 2020 [13] CHINA (Xizang*)

Sinosidus albescens (DC.) G. L. Nesom var. megaphyllus (Y. Ling) G. L. Nesom [New Combination] B.: Aster albescens (DC.) Wall. ex Hand.-Mazz. var. megaphyllus Y. Ling

NESOM Guy L., New Genera from Asian Aster (Asteraceae: Astereae). Phytoneuron (64): 1-44. 2020 [13] CHINA (Sichuan*)

Sinosidus albescens (DC.) G. L. Nesom var. pilosus (Hand.-Mazz.) G. L. Nesom [New Combination] B.: Aster albescens (DC.) Wall. ex Hand.-Mazz. var. pilosus Hand.-Mazz.

NESOM Guy L., New Genera from Asian Aster (Asteraceae: Astereae). Phytoneuron (64): 1-44. 2020 [13]

CHINA (Yunnan*) 
杜诚, 刘军, 叶文, 廖帅, 葛斌杰, 刘冰, 马金双 (2021) 中国植物新分类群、新名称 2020 年度报告. 生物多样性, 29, 10111020. http://www.biodiversity-science.net/CN/10.17520/biods.2021122

Sinosidus albescens (DC.) G. L. Nesom var. rugosus (Y. Ling) G. L. Nesom [New Combination] B.: Aster albescens (DC.) Wall. ex Hand.-Mazz. var. rugosus Y. Ling

NESOM Guy L., New Genera from Asian Aster (Asteraceae: Astereae). Phytoneuron (64): 1-44. 2020 [13] CHINA (Sichuan*)

Sinosidus albescens (DC.) G. L. Nesom var. salignus (Franch.) G. L. Nesom [New Combination] B.: Inula cuspidata (DC.) C. B. Clarke var. saligna Franch.

NESOM Guy L., New Genera from Asian Aster (Asteraceae: Astereae). Phytoneuron (64): 1-44. 2020 [13] CHINA (Xizang*)

Sinosidus argyropholis (Hand.-Mazz.) G. L. Nesom [New Combination]

B.: Aster argyropholis Hand.-Mazz.

NESOM Guy L., New Genera from Asian Aster (Asteraceae: Astereae). Phytoneuron (64): 1-44. 2020 [13]

CHINA (Sichuan*)

Sinosidus fulgidulus (Grierson) G. L. Nesom [New Combination]

B.: Aster fulgidulus Grierson

NESOM Guy L., New Genera from Asian Aster (Asteraceae: Astereae). Phytoneuron (64): 1-44. 2020 [13]

CHINA (Xizang*)

Sinosidus hypoleucus (Hand.-Mazz.) G. L. Nesom [New Combination]

B.: Aster hypoleucus Hand.-Mazz.

NESOM Guy L., New Genera from Asian Aster (Asteraceae: Astereae). Phytoneuron (64): 1-44. 2020 [13]

CHINA (Xizang*)

Sinosidus lavandulifolius (Hand.-Mazz.) G. L. Nesom [New Combination]

B.: Aster lavandulifolius Hand.-Mazz.

NESOM Guy L., New Genera from Asian Aster (Asteraceae: Astereae). Phytoneuron (64): 1-44. 2020 [14]

CHINA (Sichuan*)

Sinosidus lavandulifolius (Hand.-Mazz.) G. L. Nesom [Typification]

NESOM Guy L., New Genera from Asian Aster (Asteraceae: Astereae). Phytoneuron (64): 1-44. 2020 [14]

CHINA (Sichuan*)

Sinosidus motuoensis (Y. L. Chen) G. L. Nesom [New Combination]

B.: Aster motuoensis Y. L. Chen

NESOM Guy L., New Genera from Asian Aster (Asteraceae: Astereae). Phytoneuron (64): 1-44. 2020 [14]

CHINA (Xizang*)

Sinosidus paradoxus (Y. Ling) G. L. Nesom [New Combination]

B.: Aster argyropholis Hand.-Mazz. var. paradoxus Y. Ling

NESOM Guy L., New Genera from Asian Aster (Asteraceae: Astereae). Phytoneuron (64): 1-44. 2020 [13]

CHINA (Sichuan*)

Sinosidus polius (C. K. Schneid.) G. L. Nesom [New Combination]

B.: Aster polius C. K. Schneid.

NESOM Guy L., New Genera from Asian Aster (Asteraceae: Astereae). Phytoneuron (64): 1-44. 2020 [14]

CHINA (Sichuan*)

Synotis duclouxii (Dunn) C. Jeffrey \& Y. L. Chen [New Synonyms]

S.: Synotis longipes C. Jeffrey \& Y. L. Chen

LIU Yu-lin, ZHANG Rui, REN Chen, LIU Bing, ZHANG Yu, TANG Ming, On the Specific Identity of Chinese Endemic Species Synotis longipes (Compositae- Senecioneae). Phytotaxa 472 (3): 269-276. 2020 [271] doi: 10.11646/phytotaxa.472.3.5

CHINA (Sichuan, Yunnan*)

Synotis panzhouensis M. Tang \& H. L. Zheng 盘州合耳菊 [New Species] IUCN: CR: B+C; Evidence: Morphology

LI Zhi, ZHENG Hai-lei, TANG Ming, Synotis panzhouensis (Asteraceae, Senecioneae), a Distinct New Species with Red-purple Pappus from Southwestern Guizhou, China. PhytoKeys 166: 79-86. 2020 [80] doi: 10.3897/phytokeys.166.58654

CHINA (Guizhou*)

Taraxacum dilutissimum Kirschner \& Štěpánek [New Species] IUCN: NE; Evidence: Morphology

KIRSCHNER Jan, ŠTĚPÁNEK Jan, KLIMEŠ Leoš, DVORSKÝ Miroslav, BRŮNA Josef, MACEK Martin, KOPECKÝ Martin, The Taraxacum Flora of Ladakh, with Notes on the Adjacent Regions of the West Himalaya. Phytotaxa 457 (1): 1-409. 2020 [227] doi: 10.11646/phytotaxa.457.1.1

CHINA (Xizang); INDIA*, UZBEKISTAN 
杜诚, 刘军, 叶文, 廖帅, 葛斌杰, 刘冰, 马金双 (2021) 中国植物新分类群、新名称 2020 年度报告. 生物多样性, 29, 10111020. http://www.biodiversity-science.net/CN/10.17520/biods.2021122

Taraxacum hedinii G. Haglund ex Kirschner \& Štěpánek [New Species] IUCN: NE; Evidence: Morphology

KIRSCHNER Jan, ŠTĚPÁNEK Jan, KLIMEŠ Leoš, DVORSKÝ Miroslav, BRŮNA Josef, MACEK Martin, KOPECKÝ Martin, The Taraxacum Flora of Ladakh, with Notes on the Adjacent Regions of the West Himalaya. Phytotaxa 457 (1): 1-409. 2020 [185] doi: 10.11646/phytotaxa.457.1.1

CHINA (Xinjiang); INDIA*

Taraxacum tubulosum Kirschner \& Štěpánek [New Species] IUCN: NE; Evidence: Morphology

KIRSCHNER Jan, ŠTĚPÁNEK Jan, KLIMEŠ Leoš, DVORSKÝ Miroslav, BRŮNA Josef, MACEK Martin, KOPECKÝ Martin, The Taraxacum Flora of Ladakh, with Notes on the Adjacent Regions of the West Himalaya. Phytotaxa 457 (1): 1-409. 2020 [63] doi: 10.11646/phytotaxa.457.1.1

CHINA (Xinjiang); INDIA*

Tibetiodes G. L. Nesom [New Genus]

NESOM Guy L., Tibetiodes Nesom, gen. nov. (Asteraceae: Astereae), including 27 Species of Himalayan Aster. Phytoneuron (63): 130. $2020[2]$

CHINA

Tibetiodes angustior (Hand.-Mazz.) G. L. Nesom [New Combination]

B.: Aster yunnanensis Franch. var. angustior Hand.-Mazz.

NESOM Guy L., Tibetiodes Nesom, gen. nov. (Asteraceae: Astereae), including 27 Species of Himalayan Aster. Phytoneuron (63): 130. 2020 [2]

CHINA (Sichuan, Yunnan*)

Tibetiodes asteroides (DC.) G. L. Nesom [New Combination]

B.: Heterochaeta asteroides DC.

NESOM Guy L., Tibetiodes Nesom, gen. nov. (Asteraceae: Astereae), including 27 Species of Himalayan Aster. Phytoneuron (63): 130. 2020 [2]

CHINA (Gansu, Qinghai, Sichuan, Xizang, Yunnan); BHUTAN, INDIA, NEPAL

Tibetiodes bietii (Franch.) G. L. Nesom [New Combination]

B.: Aster bietii Franch.

NESOM Guy L., Tibetiodes Nesom, gen. nov. (Asteraceae: Astereae), including 27 Species of Himalayan Aster. Phytoneuron (63): 130. 2020 [3]

CHINA (Xizang, Yunnan*)

Tibetiodes brachytricha (Franch.) G. L. Nesom [New Combination]

B.: Aster brachytricha Franch.

NESOM Guy L., Tibetiodes Nesom, gen. nov. (Asteraceae: Astereae), including 27 Species of Himalayan Aster. Phytoneuron (63): 130. 2020 [3]

CHINA (Guizhou, Sichuan, Yunnan*); MYANMAR

Tibetiodes brachytricha (Franch.) G. L. Nesom [Typification]

NESOM Guy L., Tibetiodes Nesom, gen. nov. (Asteraceae: Astereae), including 27 Species of Himalayan Aster. Phytoneuron (63): 130. 2020 [3]

CHINA (Guizhou, Sichuan, Yunnan*); MYANMAR

Tibetiodes brevis (Hand.-Mazz.) G. L. Nesom [New Combination]

B.: Aster brevis Hand.-Mazz.

NESOM Guy L., Tibetiodes Nesom, gen. nov. (Asteraceae: Astereae), including 27 Species of Himalayan Aster. Phytoneuron (63): 130. 2020 [3]

CHINA (Yunnan*)

Tibetiodes bulleyana (Jeffrey ex Diels) G. L. Nesom [New Combination]

B.: Aster bulleyanus Jeffrey ex Diels

NESOM Guy L., Tibetiodes Nesom, gen. nov. (Asteraceae: Astereae), including 27 Species of Himalayan Aster. Phytoneuron (63): 130. 2020 [3]

CHINA (Yunnan*)

Tibetiodes diplostephioides (DC.) G. L. Nesom [New Combination]

B.: Heterochaeta diplostephioides DC.

NESOM Guy L., Tibetiodes Nesom, gen. nov. (Asteraceae: Astereae), including 27 Species of Himalayan Aster. Phytoneuron (63): 130. 2020 [3]

CHINA (Gansu, Qinghai, Sichuan, Xizang, Yunnan); BHUTAN, INDIA, NEPAL, PAKISTAN

Tibetiodes farreri (W. W. Smith \& Jeffrey) G. L. Nesom [New Combination] 
杜诚, 刘军, 叶文, 廖帅, 葛斌杰, 刘冰, 马金双 (2021) 中国植物新分类群、新名称 2020 年度报告. 生物多样性, 29, 10111020. http://www.biodiversity-science.net/CN/10.17520/biods.2021122

B.: Aster farreri W. W. Smith \& Jeffrey

NESOM Guy L., Tibetiodes Nesom, gen. nov. (Asteraceae: Astereae), including 27 Species of Himalayan Aster. Phytoneuron (63): 130. $2020[4]$

CHINA (Gansu, Hubei, Qinghai, Shanxi, Sichuan, Xizang*)

Tibetiodes farreri (W. W. Smith \& Jeffrey) G. L. Nesom [Typification]

NESOM Guy L., Tibetiodes Nesom, gen. nov. (Asteraceae: Astereae), including 27 Species of Himalayan Aster. Phytoneuron (63): 130. 2020 [4]

CHINA (Gansu, Hubei, Qinghai, Shanxi, Sichuan, Xizang*)

Tibetiodes flaccida (Bunge) G. L. Nesom [New Combination]

B.: Aster flaccidus Bunge

NESOM Guy L., Tibetiodes Nesom, gen. nov. (Asteraceae: Astereae), including 27 Species of Himalayan Aster. Phytoneuron (63): 130. $2020[4]$

CHINA (Gansu, Hebei, Qinghai, Shaanxi, Shanxi, Sichuan, Xinjiang, Xizang, Yunnan); BHUTAN, INDIA, IRAN, KASHMIR, KAZAKHSTAN, MONGOLIA, NEPAL, PAKISTAN, RUSSIA*, UZBEKISTAN

Tibetiodes glandulosa (Keissl.) G. L. Nesom [New Combination]

B.: Aster flaccidus Bunge var. glandulosus Keissl.

NESOM Guy L., Tibetiodes Nesom, gen. nov. (Asteraceae: Astereae), including 27 Species of Himalayan Aster. Phytoneuron (63): 130. $2020[4]$

CHINA (Xizang*)

Tibetiodes himalaica (C. B. Clarke) G. L. Nesom [New Combination]

B.: Aster himalaicus C. B. Clarke

NESOM Guy L., Tibetiodes Nesom, gen. nov. (Asteraceae: Astereae), including 27 Species of Himalayan Aster. Phytoneuron (63): 130. 2020 [5]

CHINA (Sichuan, Xizang, Yunnan); BHUTAN, INDIA*, MYANMAR, NEPAL

Tibetiodes himalaica (C. B. Clarke) G. L. Nesom [Typification]

NESOM Guy L., Tibetiodes Nesom, gen. nov. (Asteraceae: Astereae), including 27 Species of Himalayan Aster. Phytoneuron (63): 130. 2020 [5]

CHINA (Sichuan, Xizang, Yunnan); BHUTAN, INDIA*, MYANMAR, NEPAL

Tibetiodes jeffreyana (Diels) G. L. Nesom [New Combination]

B.: Aster jeffreyana Diels

NESOM Guy L., Tibetiodes Nesom, gen. nov. (Asteraceae: Astereae), including 27 Species of Himalayan Aster. Phytoneuron (63): 130. 2020 [5]

CHINA (Guizhou, Sichuan, Yunnan*)

Tibetiodes labrangensis (Hand.-Mazz.) G. L. Nesom [New Combination]

B.: Aster labrangensis Hand.-Mazz.

NESOM Guy L., Tibetiodes Nesom, gen. nov. (Asteraceae: Astereae), including 27 Species of Himalayan Aster. Phytoneuron (63): 130. $2020[5]$

CHINA (Gansu, Qinghai, Sichuan, Xizang*)

Tibetiodes latibracteata (Franch.) G. L. Nesom [New Combination]

B.: Aster latibracteata Franch.

NESOM Guy L., Tibetiodes Nesom, gen. nov. (Asteraceae: Astereae), including 27 Species of Himalayan Aster. Phytoneuron (63): 130. 2020 [5]

CHINA (Yunnan*); MYANMAR

Tibetiodes latisquamatum (C. E. C. Fisch.) G. L. Nesom [New Combination]

B.: Doronicum latisquamatum C. E. C. Fisch.

NESOM Guy L., Tibetiodes Nesom, gen. nov. (Asteraceae: Astereae), including 27 Species of Himalayan Aster. Phytoneuron (63): 130. 2020 [5]

CHINA (Xizang); INDIA*

Tibetiodes likiangensis (Franch.) G. L. Nesom [New Combination]

B.: Aster likiangensis Franch.

NESOM Guy L., Tibetiodes Nesom, gen. nov. (Asteraceae: Astereae), including 27 Species of Himalayan Aster. Phytoneuron (63): 130. 2020 [6]

CHINA (Sichuan, Xizang, Yunnan*); BHUTAN

Tibetiodes likiangensis (Franch.) G. L. Nesom [Typification] 
杜诚, 刘军, 叶文, 廖帅, 葛斌杰, 刘冰, 马金双 (2021) 中国植物新分类群、新名称 2020 年度报告. 生物多样性, 29, 10111020. http://www.biodiversity-science.net/CN/10.17520/biods.2021122

NESOM Guy L., Tibetiodes Nesom, gen. nov. (Asteraceae: Astereae), including 27 Species of Himalayan Aster. Phytoneuron (63): 130. 2020 [6]

CHINA (Sichuan, Xizang, Yunnan*); BHUTAN

Tibetiodes lipskii (Komarov) G. L. Nesom [New Combination]

B.: Aster lipskii Komarov

NESOM Guy L., Tibetiodes Nesom, gen. nov. (Asteraceae: Astereae), including 27 Species of Himalayan Aster. Phytoneuron (63): 130. 2020 [6]

CHINA (Gansu, Qinghai)

Tibetiodes retusa (Ludlow) G. L. Nesom [New Combination]

B.: Aster retusus Ludlow

NESOM Guy L., Tibetiodes Nesom, gen. nov. (Asteraceae: Astereae), including 27 Species of Himalayan Aster. Phytoneuron (63): 130. 2020 [6]

CHINA (Xizang*)

Tibetiodes salwinensis (Onno) G. L. Nesom [New Combination]

B.: Aster salwinensis Onno

NESOM Guy L., Tibetiodes Nesom, gen. nov. (Asteraceae: Astereae), including 27 Species of Himalayan Aster. Phytoneuron (63): 130. 2020 [6]

CHINA (Sichuan, Xizang, Yunnan*); MYANMAR

Tibetiodes salwinensis (Onno) G. L. Nesom [Typification]

NESOM Guy L., Tibetiodes Nesom, gen. nov. (Asteraceae: Astereae), including 27 Species of Himalayan Aster. Phytoneuron (63): 130. 2020 [6]

CHINA (Sichuan, Xizang, Yunnan*); MYANMAR

Tibetiodes setchuenensis (Franch.) G. L. Nesom [New Combination]

B.: Aster setchuenensis Franch.

NESOM Guy L., Tibetiodes Nesom, gen. nov. (Asteraceae: Astereae), including 27 Species of Himalayan Aster. Phytoneuron (63): 130. 2020 [6]

CHINA (Sichuan*)

Tibetiodes stracheyi (Hook. f.) G. L. Nesom [New Combination]

B.: Aster stracheyi Hook. f.

NESOM Guy L., Tibetiodes Nesom, gen. nov. (Asteraceae: Astereae), including 27 Species of Himalayan Aster. Phytoneuron (63): 130. 2020 [7]

CHINA (Xizang); BHUTAN, INDIA*, NEPAL

Tibetiodes stracheyi (Hook. f.) G. L. Nesom [Typification]

NESOM Guy L., Tibetiodes Nesom, gen. nov. (Asteraceae: Astereae), including 27 Species of Himalayan Aster. Phytoneuron (63): 130. 2020 [7]

CHINA (Xizang); BHUTAN, INDIA*, NEPAL

Tibetiodes techinensis (Y. Ling) G. L. Nesom [New Combination]

B.: Aster techinensis Y. Ling

NESOM Guy L., Tibetiodes Nesom, gen. nov. (Asteraceae: Astereae), including 27 Species of Himalayan Aster. Phytoneuron (63): 130. 2020 [7]

CHINA (Yunnan*)

Tibetiodes tientschwanensis (Hand.-Mazz.) G. L. Nesom [New Combination]

B.: Aster tientschwanensis Hand.-Mazz.

NESOM Guy L., Tibetiodes Nesom, gen. nov. (Asteraceae: Astereae), including 27 Species of Himalayan Aster. Phytoneuron (63): 130. 2020 [7]

CHINA (Sichuan*)

Tibetiodes tsarungensis (Grierson) G. L. Nesom [New Combination]

B.: Aster flaccidus Bunge subsp. tsarungensis Grierson

NESOM Guy L., Tibetiodes Nesom, gen. nov. (Asteraceae: Astereae), including 27 Species of Himalayan Aster. Phytoneuron (63): 130. 2020 [7]

CHINA (Sichuan, Xizang*, Yunnan)

Tibetiodes yunnanensis (Franch.) G. L. Nesom [New Combination]

B.: Aster yunnanensis Franch. 
杜诚, 刘军, 叶文, 廖帅, 葛斌杰, 刘冰, 马金双 (2021) 中国植物新分类群、新名称 2020 年度报告. 生物多样性, 29, 10111020. http://www.biodiversity-science.net/CN/10.17520/biods.2021122

NESOM Guy L., Tibetiodes Nesom, gen. nov. (Asteraceae: Astereae), including 27 Species of Himalayan Aster. Phytoneuron (63): 130. 2020 [7]

CHINA (Yunnan*)

Tibetiodes yunnanensis (Franch.) G. L. Nesom [Typification]

NESOM Guy L., Tibetiodes Nesom, gen. nov. (Asteraceae: Astereae), including 27 Species of Himalayan Aster. Phytoneuron (63): 130. 2020 [7]

CHINA (Yunnan*)

Tricholepis tibetica Hook. f. \& Thomson ex C. B. Clarke [Typification]

KAZEM Negaresh, Lectotypification of Tricholepis eburnea Rech. F. and T. Tibetica Hook. F. \& Thomson ex C.B. Clarke (Asteraceae). Annales Botanici Fennici 57 (1-3): 177-180. 2020 [177] doi: 10.5735/085.057.0124

CHINA (Xizang); INDIA*

Vickifunkia C. Ren, Long Wang, I. D. Illar. \& Q. E. Yang [New Name]

R.: Ligularia sect. Stenostegia Pojark.

REN Chen, WANG Long, ILLARIONOVA Irina D., YANG Qin-er, Circumscription and Phylogenetic Position of Ligularia sect. Stenostegia (Asteraceae: Senecioneae) Based on Morphological, Cytological, and Molecular Phylogenetic Evidence. Taxon 69 (4): 739-755. 2020 [748] doi: 10.1002/tax.12280

CHINA

Vickifunkia narynensis (C. Winkl.) C. Ren, Long Wang, I. D. Illar. \& Q. E. Yang [New Combination]

B.: Senecio narynensis C. Winkl.

REN Chen, WANG Long, ILLARIONOVA Irina D., YANG Qin-er, Circumscription and Phylogenetic Position of Ligularia sect. Stenostegia (Asteraceae: Senecioneae) Based on Morphological, Cytological, and Molecular Phylogenetic Evidence. Taxon 69 (4): 739-755. 2020 [749] doi: 10.1002/tax.12280

CHINA (Xinjiang); KYRGYZSTAN*

Vickifunkia schischkinii (Rubtz.) C. Ren, Long Wang, I. D. Illar. \& Q. E. Yang [New Combination]

B.: Ligularia schischkinii Rubtz.

REN Chen, WANG Long, ILLARIONOVA Irina D., YANG Qin-er, Circumscription and Phylogenetic Position of Ligularia sect. Stenostegia (Asteraceae: Senecioneae) Based on Morphological, Cytological, and Molecular Phylogenetic Evidence. Taxon 69 (4): 739-755. 2020 [749] doi: 10.1002/tax.12280

CHINA (Xinjiang); KAZAKHSTAN*

Vickifunkia songarica (Fisch.) C. Ren, Long Wang, I. D. Illar. \& Q. E. Yang [New Combination]

B.: Senecio songaricus Fisch.

REN Chen, WANG Long, ILLARIONOVA Irina D., YANG Qin-er, Circumscription and Phylogenetic Position of Ligularia sect. Stenostegia (Asteraceae: Senecioneae) Based on Morphological, Cytological, and Molecular Phylogenetic Evidence. Taxon 69 (4): 739-755. 2020 [749] doi: 10.1002/tax.12280

CHINA (Xinjiang); KAZAKHSTAN*

Vickifunkia thomsonii (C. B. Clarke) C. Ren, Long Wang, I. D. Illar. \& Q. E. Yang [New Combination]

B.: Senecio thomsonii C. B. Clarke

REN Chen, WANG Long, ILLARIONOVA Irina D., YANG Qin-er, Circumscription and Phylogenetic Position of Ligularia sect. Stenostegia (Asteraceae: Senecioneae) Based on Morphological, Cytological, and Molecular Phylogenetic Evidence. Taxon 69 (4): 739-755. 2020 [749] doi: 10.1002/tax.12280

CHINA (Xinjiang); INDIA

Vickifunkia thomsonii (C. B. Clarke) C. Ren, Long Wang, I. D. Illar. \& Q. E. Yang [Typification]

REN Chen, WANG Long, ILLARIONOVA Irina D., YANG Qin-er, Circumscription and Phylogenetic Position of Ligularia sect. Stenostegia (Asteraceae: Senecioneae) Based on Morphological, Cytological, and Molecular Phylogenetic Evidence. Taxon 69 (4): 739-755. 2020 [749] doi: 10.1002/tax.12280

CHINA (Xinjiang); INDIA

Vickifunkia tianschanica (Chang Y. Yang \& S. L. Keng) C. Ren, Long Wang, I. D. Illar. \& Q. E. Yang [New Combination]

B.: Ligularia tianschanica Chang Y. Yang \& S. L. Keng

REN Chen, WANG Long, ILLARIONOVA Irina D., YANG Qin-er, Circumscription and Phylogenetic Position of Ligularia sect. Stenostegia (Asteraceae: Senecioneae) Based on Morphological, Cytological, and Molecular Phylogenetic Evidence. Taxon 69 (4): 739-755. 2020 [749] doi: 10.1002/tax.12280

CHINA (Xinjiang*)

Yonglingia G. L. Nesom [New Genus]

NESOM Guy L., Yonglingia, Chaochienchangia, and Sinobouffordia (Asteraceae: Astereae), New Genera Segregated from Aster in China. Phytoneuron (57): 1-25. 2020 [1] CHINA 
杜诚, 刘军, 叶文, 廖帅, 葛斌杰, 刘冰, 马金双 (2021) 中国植物新分类群、新名称 2020 年度报告. 生物多样性, 29, 10111020. http://www.biodiversity-science.net/CN/10.17520/biods.2021122

Yonglingia hersileoides (C. K. Schneid.) G. L. Nesom [New Combination]

B.: Aster hersileoides C. K. Schneid.

NESOM Guy L., Yonglingia, Chaochienchangia, and Sinobouffordia (Asteraceae: Astereae), New Genera Segregated from Aster in

China. Phytoneuron (57): 1-25. 2020 [2]

CHINA (Sichuan*)

Yonglingia nitida (C. C. Chang) G. L. Nesom [New Combination]

B.: Aster nitidus C. C. Chang

NESOM Guy L., Yonglingia, Chaochienchangia, and Sinobouffordia (Asteraceae: Astereae), New Genera Segregated from Aster in China. Phytoneuron (57): 1-25. 2020 [2]

CHINA (Chongqing*, Sichuan)

\section{Balsaminaceae 凤仙花科}

Impatiens bomiensis Y. Y. Cong \& Y. C. Peng 波密凤仙花 [New Species] IUCN: NE; Evidence: ITS, atpB-rbcL; Morphology PENG Yang-cheng, PENG Shuai, YANG Ning, XU Zhi-gao, CONG Yi-yan, Impatiens bomiensis (Balsaminaceae), a New Species from Xizang, China. Nordic Journal of Botany 38 (e02761): 1-5. 2020 [2] doi: 10.1111/njb.02761

CHINA (Xizang*)

Impatiens dasyvexilla Q. L. Gan \& Xin W. Li [New Species] IUCN: NE; Evidence: Morphology

GAN Qi-liang, LI Xin-wei, Impatiens dasyvexilla, a New Species of Impatiens (Balsaminaceae) from Hubei, China. Novon 28 (4): 253-255. 2020 [253] doi: 10.3417/2020578

CHINA (Hubei*)

Impatiens gongchengensis Z. C. Lu, B. Pan \& Yan Liu 恭城凤仙花 [New Species] IUCN: NE; Evidence: Morphology

LU Zhao-cen, PAN Bo, HUANG Fu-zhao, LIU Yan, Impatiens gongchengensis (Balsaminaceae), a New Species from Guangxi, Southern China. Taiwania 65 (1): 1-4. 2020 [1] doi: 10.6165/tai.2020.65.1

CHINA (Guangxi*)

Impatiens longipes Hook. f. \& Thomson [Typification]

GOGOI Rajib, SHERPA Norbu, BORAH Souravjyoti, Taxonomic Note on Impatiens longipes (Balsaminaceae) and Description of the New Species I. pseudolongipes. Phytotaxa 429 (3): 217-224. 2020 [218] doi: 10.11646/phytotaxa.429.3.3

CHINA (Xizang); BHUTAN, INDIA*

Impatiens longlinensis S. X. Yu [New Species] IUCN: CR; Evidence: ITS, atpB-rbcL, trnL-F; Morphology

XUE Tian-tian, XIA Chang-ying, LIDÉN Magnus, XU Wei-bin, LU Zhao-cen, CHEN Hai-ling, LI Shu-wan, YU Sheng-xiang, Ignored Biodiversity in Acid Soil Islands in Karst Areas, South China: Impatiens longlinensis (Balsaminaceae), a New Critically Endangered Species. Systematic Botany 45 (4): 891-899. 2020 [894] doi: 10.1600/036364420X16033962925222

CHINA (Guangxi*)

Impatiens macrantha S. X. Yu \& Ying Qin [New Species] IUCN: NE; Evidence: ITS, atpB-rbcL, trnL-F; Morphology

QIN Ying, XIA Chang-ying, YANG Xu-dong, TRIBOUN Pramote, SON Hoang Thanh, WU Xue-xue, PEI Zi-xin, YU Sheng-xiang, Impatiens macrantha (Balsaminaceae) sp. nov., a New Species from Limestone Areas in Guangxi, China. Nordic Journal of Botany 38 (e02784): 1-7. 2020 [2] doi: 10.1111/njb.02784

CHINA (Guangxi*)

Impatiens plicatisepala C. Y. Zou, Yan Liu \& S. X. Yu 褶蓦凤仙花 [New Species] IUCN: NE; Evidence: Morphology

ZOU Chun-yu, LIU Yan, LI Jin, YU Sheng-xiang, Impatiens plicatisepala (Balsaminaceae), a New Species from Guangxi, China. Taiwania 65 (4): 544-547. 2020 [544] doi: 10.6165/tai.2020.65.544

CHINA (Guangxi*)

Impatiens porrecta Wall. ex Hook. f. \& Thomson 伸展凤仙花 [New Record]

ZHANG Cai-fei, PENG Shuai, TIAN Jing, HU Guang-wan, WANG Qing-feng, a New Species and a Newly Recorded Species of Impatiens (Balsaminaceae) from Yunnan, China. Plant Science Journal 38 (4): 437-447. 2020 [444] doi: 10.11913/PSJ.20950837.2020.40437

CHINA (Xizang, Yunnan); BANGLADESH*, INDIA, LAOS, MYANMAR, NEPAL

Impatiens pseudolongipes Gogoi, Sherpa \& Borah [New Species] IUCN: NE; Evidence: Morphology

GOGOI Rajib, SHERPA Norbu, BORAH Souravjyoti, Taxonomic Note on Impatiens longipes (Balsaminaceae) and Description of the New Species I. pseudolongipes. Phytotaxa 429 (3): 217-224. 2020 [218] doi: 10.11646/phytotaxa.429.3.3

CHINA (Xizang*)

Impatiens quintadecimacopii G. W. Hu \& Q. F. Wang 滇红凤仙花 [New Species] IUCN: NE; Evidence: ITS, atpB-rbcL; Morphology 
杜诚, 刘军, 叶文, 廖帅, 葛斌杰, 刘冰, 马金双 (2021) 中国植物新分类群、新名称 2020 年度报告. 生物多样性, 29, 10111020. http://www.biodiversity-science.net/CN/10.17520/biods.2021122

ZHANG Cai-fei, PENG Shuai, TIAN Jing, HU Guang-wan, WANG Qing-feng, A New Species and a Newly Recorded Species of Impatiens (Balsaminaceae) from Yunnan, China. Plant Science Journal 38 (4): 437-447. 2020 [440] doi: 10.11913/PSJ.20950837.2020.40437

CHINA (Yunnan*)

Impatiens shenglanii Q. L. Gan \& Xin W. Li [New Species] IUCN: NE; Evidence: ITS, atpB-rbcL, trnL-F; Morphology

GAN Qi-liang, KE Zun-wei, LI Xin-wei, Impatiens shenglanii (Balsaminaceae), a New Species from Central China and Its Phylogenetic Position. Annales Botanici Fennici 57 (4-6): 217-221. 2020 [218] doi: 10.5735/085.057.0404

CHINA (Hubei*)

Impatiens shiyomiensis Hareesh \& M. Sabu [New Species] IUCN: NE; Evidence: Morphology

HAREESH Vadakkoot Sankaran, SABU Mamiyil, Two New Balsams (Balsaminaceae) from Eastern Himalayas, India. Phytotaxa 437 (5): 291-300. 2020 [294] doi: 10.11646/phytotaxa.437.5.3

CHINA (Xizang*)

Impatiens tirbinensis Hareesh \& M. Sabu [New Species] IUCN: NE; Evidence: Morphology

HAREESH Vadakkoot Sankaran, SABU Mamiyil, Two New Balsams (Balsaminaceae) from Eastern Himalayas, India. Phytotaxa 437 (5): 291-300. 2020 [291] doi: 10.11646/phytotaxa.437.5.3

CHINA (Xizang*)

Impatiens tripetala Roxb. \& DC. 三瓣凤仙花 [New Record]

ZHANG Qin-you, PENG Shuai, PENG Yang-cheng, CAI Xiu-zhen, CONG Yi-yan, Impatiens tripetala (Balsaminaceae), a New Record Species of Impatiens from China. Plant Science Journal 38 (3): 320-322. 2020 [321] doi: 10.11913/PSJ.2095-0837.2020.30320

CHINA (Yunnan); BHUTAN, INDIA

Impatiens wuyiensis Jian S. Wang, Y. F. Lu \& X. F. Jin 武夷凤仙花 [New Species] IUCN: NE; Evidence: ITS, atpB-rbcL, trnL-F; Morphology

WANG Jian-sheng, LU Yi-fei, XU Yue-liang, JIN Shui-hu, JIN Xiao-feng, Impatiens wuyiensis (Balsaminaceae), a New Species from Fujian of Southeast China, Based on Morphological and Molecular Evidences. Botanical Studies (Taipei) 61 (29): 1-12. 2020 [3] doi: 10.1186/s40529-020-00306-1

CHINA (Fujian*)

Impatiens zhuxiensis Q. L. Gan \& Xin W. Li [New Species] IUCN: NE; Evidence: ITS, atpB-rbcL, trnL-F; Morphology

GAN Qi-liang, LI Xin-wei, Impatiens zhuxiensis (Balsaminaceae), a New Species of Hubei, China. Nordic Journal of Botany 38 (e02686): 1-5. 2020 [2] doi: 10.1111/njb.02686

CHINA (Hubei*)

\section{Begoniaceae 秋海棠科}

Begonia aurora C. I Peng, Yan Liu \& W. B. Xu 极光秋海棠 [New Species] IUCN: NE; Evidence: ndhA, ndhF-rpl32, rpl32-trnL; Morphology

LIU Yan, TSENG Yu-hsin, YANG Hsun-an, HU Ai-qun, XU Wei-bin, LIN Che-wei, KONO Yoshiko, CHANG Chiung-chih, PENG Ching-i, CHUNG Kuo-fang, Six New Species of Begonia from Guangxi, China. Botanical Studies (Taipei) 61 (21): 1-23. 2020 [3] doi: 10.1186/s40529-020-00298-y

CHINA (Guangxi*)

Begonia circumlobata Hance [New Synonyms]

S.: Begonia lipingensis Irmsch.

TIAN Dai-ke, XIAO Yan, LI Yan-ci, YAN Ke-jian, Several New Records, Synonyms, and Hybrid-origin of Chinese Begonias. PhytoKeys 153: 13-35. 2020 [18] doi: 10.3897/phytokeys.153.50805

CHINA (Guangdong*, Guizhou)

Begonia depingiana Y. H. Tan \& H. B. Ding [New Species] IUCN: VU: D2; Evidence: Morphology

DING Hong-bo, GONG Yan-xiong, PAN Rui, LU Xiao-qiang, TAN Yun-hong, A New Tuberous Species of Begonia L. (Begoniaceae) from Southern Yunnan, China. Phytotaxa 474 (1): 81-86. 2020 [82] doi: 10.11646/phytotaxa.474.1.8

CHINA (Yunnan*)

Begonia dioica Buch.-Ham. ex D. Don 走茎秋海棠 [New Record]

TIAN Dai-ke, XIAO Yan, LI Yan-ci, YAN Ke-jian, Several New Records, Synonyms, and Hybrid-origin of Chinese Begonias. PhytoKeys 153: 13-35. 2020 [14] doi: 10.3897/phytokeys.153.50805

CHINA (Xizang); BHUTAN, INDIA, NEPAL, PAKISTAN

Begonia flagellaris H. Hara 鞭状秋海棠 [New Record]

TIAN Dai-ke, XIAO Yan, LI Yan-ci, YAN Ke-jian, Several New Records, Synonyms, and Hybrid-origin of Chinese Begonias. PhytoKeys 153: 13-35. 2020 [16] doi: 10.3897/phytokeys.153.50805 
杜诚, 刘军, 叶文, 廖帅, 葛斌杰, 刘冰, 马金双 (2021) 中国植物新分类群、新名称 2020 年度报告. 生物多样性, 29, 10111020. http://www.biodiversity-science.net/CN/10.17520/biods.2021122

CHINA (Xizang); NEPAL

Begonia guangdongensis W. H. Tu, B. M. Wang \& Yu L. Li [New Species] IUCN: CR: B2ab(iii); Evidence: Morphology

TU Wen-hui, WANG Bing-mou, HUANG Yi, YAO Gang, HUANG Jiu-xiang, LI Yu-ling, Begonia guangdongensis, a New Species of

Begonia (Begoniaceae) from Guangdong, China. PhytoKeys 162: 29-36. 2020 [30] doi: 10.3897/phytokeys.162.51913

CHINA (Guangdong*)

Begonia larvata C. I Peng, Yan Liu \& W. B. Xu 果子狸秋海棠 [New Species] IUCN: NE; Evidence: ndhA, ndhF-rpl32, rpl32-trnL; Morphology

LIU Yan, TSENG Yu-hsin, YANG Hsun-an, HU Ai-qun, XU Wei-bin, LIN Che-wei, KONO Yoshiko, CHANG Chiung-chih, PENG Ching-i, CHUNG Kuo-fang, Six New Species of Begonia from Guangxi, China. Botanical Studies (Taipei) 61 (21): 1-23. 2020 [7] doi: 10.1186/s40529-020-00298-y

CHINA (Guangxi*)

Begonia longiciliata C. Y. Wu [New Synonyms]

S.: Begonia sizemoreae Kiew (R. Kiew 5304, holotype: SING; isotype: HN)

TIAN Dai-ke, XIAO Yan, LI Yan-ci, YAN Ke-jian, Several New Records, Synonyms, and Hybrid-origin of Chinese Begonias. PhytoKeys 153: 13-35. 2020 [21] doi: 10.3897/phytokeys.153.50805

CHINA (Guangxi, Guizhou*, Yunnan); LAOS, VIET NAM

Begonia longiornithophylla C. I Peng, W. B. Xu \& Yan Liu 长茎鸟叶秋海棠［New Species] IUCN: NE; Evidence: ndhA, ndhF-rpl32, rpl32-trnL; Chromosome; Morphology

LIU Yan, TSENG Yu-hsin, YANG Hsun-an, HU Ai-qun, XU Wei-bin, LIN Che-wei, KONO Yoshiko, CHANG Chiung-chih, PENG Ching-i, CHUNG Kuo-fang, Six New Species of Begonia from Guangxi, China. Botanical Studies (Taipei) 61 (21): 1-23. 2020 [10] doi: 10.1186/s40529-020-00298-y

CHINA (Guangxi*)

Begonia lui S. M. Ku, C. I Peng \& Yan Liu 陆氏秋海棠 [New Species] IUCN: NE; Evidence: ndhA, ndhF-rpl32, rpl32-trnL; Morphology

LIU Yan, TSENG Yu-hsin, YANG Hsun-an, HU Ai-qun, XU Wei-bin, LIN Che-wei, KONO Yoshiko, CHANG Chiung-chih, PENG Ching-i, CHUNG Kuo-fang, Six New Species of Begonia from Guangxi, China. Botanical Studies (Taipei) 61 (21): 1-23. 2020 [13] doi: 10.1186/s40529-020-00298-y

CHINA (Guangxi*)

Begonia manhaoensis S. H. Huang \& Y. M. Shui [New Synonyms]

S.: Begonia naga N. Krishna \& Pradee

AUNG Aung, CHEN Wen-hong, SHUI Yu-min, Begonia naga, a Synonym of B. manhaoensis (Begoniaceae). Phytotaxa 429 (4): $297-$ 300. 2020 [297] doi: 10.11646/phytotaxa.429.4.4

CHINA (Yunnan*); INDIA

Begonia mengdongensis H. H. Xi 猛硐秋海棠 [New Species] IUCN: CR: B2b(iii, iv, v); C2a(i); Evidence: Morphology

XI Hui-hui, XIAO Si-yue, WANG Yi-qing, XIAO Bo, WANG Wen-guang, Begonia mengdongensis, a New Tuberous Species of Begonia (Begoniaceae) from Southeastern Yunnan, China. Nordic Journal of Botany 38 (e02645): 1-7. 2020 [2] doi: 10.1111/njb.02645

CHINA (Yunnan*)

Begonia oyuniae Taram \& N. Krishna [New Species] IUCN: NE; Evidence: Morphology

TARAM M., BORAH D., KRISHNA N., PRADEEP A. K., AMRUTHA A., HUGHES M., Begonia oyuniae (Begonia sect. Monophyllon, Begoniaceae), a Remarkable New Species from Northeast India. The Gardens' Bulletin Singapore 72 (1): $109-115$. 2020 [110] doi: 10.26492/gbs72(1).2020-10

CHINA (Xizang*)

Begonia puerensis W. G. Wang, X. D. Ma \& J. Y. Shen 普洱秋海棠 [New Species] IUCN: EN: B2a;C2a(i); Evidence: Morphology WANG Wen-guang, MA Xing-da, LI Ren-kun, SHI Ji-pu, ZHANG Shou-zhou, SHEN Jian-yong, Begonia puerensis sp. nov. (Begoniaceae), a New Tuberous Species from Yunnan, China. Nordic Journal of Botany 38 (e02618): 1-6. 2020 [2] doi: 10.1111/njb.02618

CHINA (Yunnan*)

Begonia scabrifolia C. I Peng, Yan Liu \& C. W. Lin 涩叶秋海棠 [New Species] IUCN: NE; Evidence: ndhA, ndhF-rpl32, rpl32-trnL; Morphology

LIU Yan, TSENG Yu-hsin, YANG Hsun-an, HU Ai-qun, XU Wei-bin, LIN Che-wei, KONO Yoshiko, CHANG Chiung-chih, PENG Ching-i, CHUNG Kuo-fang, Six New Species of Begonia from Guangxi, China. Botanical Studies (Taipei) 61 (21): 1-23. 2020 [16] doi: 10.1186/s40529-020-00298-y

CHINA (Guangxi*) 
杜诚, 刘军, 叶文, 廖帅, 葛斌杰, 刘冰, 马金双 (2021) 中国植物新分类群、新名称 2020 年度报告. 生物多样性, 29, 10111020. http://www.biodiversity-science.net/CN/10.17520/biods.2021122

Begonia taliensis Gagnep. [New Synonyms]

S.: Begonia muliensis T. T. Yu

TIAN Dai-ke, XIAO Yan, LI Yan-ci, YAN Ke-jian, Several New Records, Synonyms, and Hybrid-origin of Chinese Begonias. PhytoKeys 153: 13-35. 2020 [25] doi: 10.3897/phytokeys.153.50805

CHINA (Sichuan, Yunnan*)

Begonia taliensis Gagnep. [Typification]

TIAN Dai-ke, XIAO Yan, LI Yan-ci, YAN Ke-jian, Several New Records, Synonyms, and Hybrid-origin of Chinese Begonias. PhytoKeys 153: 13-35. 2020 [25] doi: 10.3897/phytokeys.153.50805

CHINA (Sichuan, Yunnan*)

Begonia xishuangbannaensis W. G. Wang \& Li J. Jiang [New Species] IUCN: NE; Evidence: Morphology

WANG Wen-guang, JIANG Li-ju, HE Kai-hong, SHI Ji-pu, SHEN Jian-yong, Begonia xishuangbannaensis (Begoniaceae), a New Tuberous Species from Yunnan, China. Annales Botanici Fennici 57 (4-6): 249-253. 2020 [249] doi: 10.5735/085.057.0409

CHINA (Yunnan*)

Begonia zhuoyuniae C. I Peng, Yan Liu \& K. F. Chung 倬云秋海棠 [New Species] IUCN: NE; Evidence: ndhA, ndhF-rpl32, rpl32trnL; Chromosome; Morphology

LIU Yan, TSENG Yu-hsin, YANG Hsun-an, HU Ai-qun, XU Wei-bin, LIN Che-wei, KONO Yoshiko, CHANG Chiung-chih, PENG Ching-i, CHUNG Kuo-fang, Six New Species of Begonia from Guangxi, China. Botanical Studies (Taipei) 61 (21): 1-23. 2020 [16] doi: 10.1186/s40529-020-00298-y

CHINA (Guangxi*)

\section{Berberidaceae 小檗科}

Berberis baiyuensis Harber [New Species] IUCN: NE; Evidence: Morphology

HARBER Julian, The Berberis of China and Vietnam, a Revision. Monographs in Systematic Botany from the Missouri Botanical Garden Volume 136: 1-360. 2020 [140] doi: 9781935641186

CHINA (Sichuan*)

Berberis barkamensis Harber [New Species] IUCN: NE; Evidence: Morphology

HARBER Julian, The Berberis of China and Vietnam, a Revision. Monographs in Systematic Botany from the Missouri Botanical Garden Volume 136: 1-360. 2020 [140] doi: 9781935641186

CHINA (Sichuan*)

Berberis basumchuensis Harber [New Species] IUCN: NE; Evidence: Morphology

HARBER Julian, The Berberis of China and Vietnam, a Revision. Monographs in Systematic Botany from the Missouri Botanical Garden Volume 136: 1-360. 2020 [141] doi: 9781935641186

CHINA (Xizang*)

Berberis baxoiensis Harber [New Species] IUCN: NE; Evidence: Morphology

HARBER Julian, The Berberis of China and Vietnam, a Revision. Monographs in Systematic Botany from the Missouri Botanical Garden Volume 136: 1-360. 2020 [142] doi: 9781935641186

CHINA (Xizang*)

Berberis biguensis Harber [New Species] IUCN: NE; Evidence: Morphology

HARBER Julian, The Berberis of China and Vietnam, a Revision. Monographs in Systematic Botany from the Missouri Botanical Garden Volume 136: 1-360. 2020 [142] doi: 9781935641186

CHINA (Yunnan*)

Berberis bouffordii Harber [New Species] IUCN: NE; Evidence: Morphology

HARBER Julian, The Berberis of China and Vietnam, a Revision. Monographs in Systematic Botany from the Missouri Botanical Garden Volume 136: 1-360. 2020 [144] doi: 9781935641186 CHINA (Qinghai*)

Berberis cornuta Harber [New Species] IUCN: NE; Evidence: Morphology

HARBER Julian, The Berberis of China and Vietnam, a Revision. Monographs in Systematic Botany from the Missouri Botanical Garden Volume 136: 1-360. 2020 [164] doi: 9781935641186

CHINA (Xizang*)

Berberis dahaiensis Harber [New Species] IUCN: NE; Evidence: Morphology

HARBER Julian, The Berberis of China and Vietnam, a Revision. Monographs in Systematic Botany from the Missouri Botanical Garden Volume 136: 1-360. 2020 [165] doi: 9781935641186 
杜诚, 刘军, 叶文, 廖帅, 葛斌杰, 刘冰, 马金双 (2021) 中国植物新分类群、新名称 2020 年度报告. 生物多样性, 29, 10111020. http://www.biodiversity-science.net/CN/10.17520/biods.2021122

\section{CHINA (Yunnan*)}

Berberis deqenensis Harber [New Species] IUCN: NE; Evidence: Morphology

HARBER Julian, The Berberis of China and Vietnam, a Revision. Monographs in Systematic Botany from the Missouri Botanical Garden Volume 136: 1-360. 2020 [171] doi: 9781935641186

CHINA (Yunnan*)

Berberis difficilis Harber [New Species] IUCN: NE; Evidence: Morphology

HARBER Julian, The Berberis of China and Vietnam, a Revision. Monographs in Systematic Botany from the Missouri Botanical Garden Volume 136: 1-360. 2020 [176] doi: 9781935641186

CHINA (Sichuan*)

Berberis dulongjiangensis Harber [New Species] IUCN: NE; Evidence: Morphology

HARBER Julian, The Berberis of China and Vietnam, a Revision. Monographs in Systematic Botany from the Missouri Botanical Garden Volume 136: 1-360. 2020 [180] doi: 9781935641186

CHINA (Yunnan*)

Berberis ebianensis Harber [New Species] IUCN: NE; Evidence: Morphology

HARBER Julian, The Berberis of China and Vietnam, a Revision. Monographs in Systematic Botany from the Missouri Botanical Garden Volume 136: 1-360. 2020 [61] doi: 9781935641186

CHINA (Sichuan*)

Berberis elliptifolia Harber [New Species] IUCN: NE; Evidence: Morphology

HARBER Julian, The Berberis of China and Vietnam, a Revision. Monographs in Systematic Botany from the Missouri Botanical Garden Volume 136: 1-360. 2020 [180] doi: 9781935641186

CHINA (Qinghai*)

Berberis emeishanensis Harber [New Species] IUCN: NE; Evidence: Morphology

HARBER Julian, The Berberis of China and Vietnam, a Revision. Monographs in Systematic Botany from the Missouri Botanical Garden Volume 136: 1-360. 2020 [181] doi: 9781935641186

CHINA (Sichuan*)

Berberis epedicellata Harber [New Species] IUCN: NE; Evidence: Morphology

HARBER Julian, The Berberis of China and Vietnam, a Revision. Monographs in Systematic Botany from the Missouri Botanical Garden Volume 136: 1-360. 2020 [182] doi: 9781935641186

CHINA (Sichuan*)

Berberis exigua Harber [New Species] IUCN: NE; Evidence: Morphology

HARBER Julian, The Berberis of China and Vietnam, a Revision. Monographs in Systematic Botany from the Missouri Botanical Garden Volume 136: 1-360. 2020 [184] doi: 9781935641186

CHINA (Yunnan*)

Berberis gaoshanensis Harber [New Species] IUCN: NE; Evidence: Morphology

HARBER Julian, The Berberis of China and Vietnam, a Revision. Monographs in Systematic Botany from the Missouri Botanical Garden Volume 136: 1-360. 2020 [188] doi: 9781935641186

CHINA (Sichuan*)

Berberis gongshanensis Harber [New Species] IUCN: NE; Evidence: Morphology

HARBER Julian, The Berberis of China and Vietnam, a Revision. Monographs in Systematic Botany from the Missouri Botanical Garden Volume 136: 1-360. 2020 [192] doi: 9781935641186

CHINA (Yunnan*)

Berberis gyaitangensis Harber [New Species] IUCN: NE; Evidence: Morphology

HARBER Julian, The Berberis of China and Vietnam, a Revision. Monographs in Systematic Botany from the Missouri Botanical Garden Volume 136: 1-360. 2020 [192] doi: 9781935641186

CHINA (Yunnan*)

Berberis heishuiensis Harber [New Species] IUCN: NE; Evidence: Morphology

HARBER Julian, The Berberis of China and Vietnam, a Revision. Monographs in Systematic Botany from the Missouri Botanical Garden Volume 136: 1-360. 2020 [194] doi: 9781935641186

CHINA (Sichuan*)

Berberis hubianensis Harber [New Species] IUCN: NE; Evidence: Morphology

HARBER Julian, The Berberis of China and Vietnam, a Revision. Monographs in Systematic Botany from the Missouri Botanical Garden Volume 136: 1-360. 2020 [199] doi: 9781935641186 
杜诚, 刘军, 叶文, 廖帅, 葛斌杰, 刘冰, 马金双 (2021) 中国植物新分类群、新名称 2020 年度报告. 生物多样性, 29, 10111020. http://www.biodiversity-science.net/CN/10.17520/biods.2021122

\section{CHINA (Yunnan*)}

Berberis kangwuensis Harber [New Species] IUCN: NE; Evidence: Morphology

HARBER Julian, The Berberis of China and Vietnam, a Revision. Monographs in Systematic Botany from the Missouri Botanical Garden Volume 136: 1-360. 2020 [208] doi: 9781935641186

CHINA (Sichuan*)

Berberis lhunzensis Harber [New Species] IUCN: NE; Evidence: Morphology

HARBER Julian, The Berberis of China and Vietnam, a Revision. Monographs in Systematic Botany from the Missouri Botanical Garden Volume 136: 1-360. 2020 [213] doi: 9781935641186

CHINA (Xizang*)

Berberis lhunzhubensis Harber [New Species] IUCN: NE; Evidence: Morphology

HARBER Julian, The Berberis of China and Vietnam, a Revision. Monographs in Systematic Botany from the Missouri Botanical Garden Volume 136: 1-360. 2020 [214] doi: 9781935641186

CHINA (Xizang*)

Berberis lixianensis Harber [New Species] IUCN: NE; Evidence: Morphology

HARBER Julian, The Berberis of China and Vietnam, a Revision. Monographs in Systematic Botany from the Missouri Botanical Garden Volume 136: 1-360. 2020 [215] doi: 9781935641186

CHINA (Sichuan*)

Berberis longipedicellata Harber [New Species] IUCN: NE; Evidence: Morphology

HARBER Julian, The Berberis of China and Vietnam, a Revision. Monographs in Systematic Botany from the Missouri Botanical Garden Volume 136: 1-360. 2020 [215] doi: 9781935641186

CHINA (Sichuan*)

Berberis mabiluoensis Harber [New Species] IUCN: NE; Evidence: Morphology

HARBER Julian, The Berberis of China and Vietnam, a Revision. Monographs in Systematic Botany from the Missouri Botanical Garden Volume 136: 1-360. 2020 [217] doi: 9781935641186

CHINA (Yunnan*)

Berberis markamensis Harber [New Species] IUCN: NE; Evidence: Morphology

HARBER Julian, The Berberis of China and Vietnam, a Revision. Monographs in Systematic Botany from the Missouri Botanical Garden Volume 136: 1-360. 2020 [218] doi: 9781935641186

CHINA (Xizang*)

Berberis monticola Harber [New Species] IUCN: NE; Evidence: Morphology

HARBER Julian, The Berberis of China and Vietnam, a Revision. Monographs in Systematic Botany from the Missouri Botanical Garden Volume 136: 1-360. 2020 [223] doi: 9781935641186

CHINA (Yunnan*)

Berberis nanifolia Harber [New Species] IUCN: NE; Evidence: Morphology

HARBER Julian, The Berberis of China and Vietnam, a Revision. Monographs in Systematic Botany from the Missouri Botanical Garden Volume 136: 1-360. 2020 [227] doi: 9781935641186

CHINA (Yunnan*)

Berberis ngawaica Harber [New Species] IUCN: NE; Evidence: Morphology

HARBER Julian, The Berberis of China and Vietnam, a Revision. Monographs in Systematic Botany from the Missouri Botanical Garden Volume 136: 1-360. 2020 [228] doi: 9781935641186

CHINA (Sichuan*)

Berberis ninglangensis Harber [New Species] IUCN: NE; Evidence: Morphology

HARBER Julian, The Berberis of China and Vietnam, a Revision. Monographs in Systematic Botany from the Missouri Botanical Garden Volume 136: 1-360. 2020 [229] doi: 9781935641186

CHINA (Yunnan*)

Berberis nujiangensis Harber [New Species] IUCN: NE; Evidence: Morphology

HARBER Julian, The Berberis of China and Vietnam, a Revision. Monographs in Systematic Botany from the Missouri Botanical Garden Volume 136: 1-360. 2020 [92] doi: 9781935641186

CHINA (Xizang*)

Berberis nyingchiensis Harber [New Species] IUCN: NE; Evidence: Morphology

HARBER Julian, The Berberis of China and Vietnam, a Revision. Monographs in Systematic Botany from the Missouri Botanical Garden Volume 136: 1-360. 2020 [229] doi: 9781935641186

CHINA (Xizang*) 
杜诚, 刘军, 叶文, 廖帅, 葛斌杰, 刘冰, 马金双 (2021) 中国植物新分类群、新名称 2020 年度报告. 生物多样性, 29, 10111020. http://www.biodiversity-science.net/CN/10.17520/biods.2021122

Berberis pluvisylvatica Harber [New Species] IUCN: NE; Evidence: Morphology

HARBER Julian, The Berberis of China and Vietnam, a Revision. Monographs in Systematic Botany from the Missouri Botanical Garden Volume 136: 1-360. 2020 [234] doi: 9781935641186

CHINA (Xizang*)

Berberis polybotrys Harber [New Species] IUCN: NE; Evidence: Morphology

HARBER Julian, The Berberis of China and Vietnam, a Revision. Monographs in Systematic Botany from the Missouri Botanical Garden Volume 136: 1-360. 2020 [235] doi: 9781935641186

CHINA (Yunnan*)

Berberis pratensis Harber [New Species] IUCN: NE; Evidence: Morphology

HARBER Julian, The Berberis of China and Vietnam, a Revision. Monographs in Systematic Botany from the Missouri Botanical Garden Volume 136: 1-360. 2020 [238] doi: 9781935641186

CHINA (Sichuan*)

Berberis pruinosifolia Harber [New Species] IUCN: NE; Evidence: Morphology

HARBER Julian, The Berberis of China and Vietnam, a Revision. Monographs in Systematic Botany from the Missouri Botanical Garden Volume 136: 1-360. 2020 [240] doi: 9781935641186

CHINA (Sichuan*)

Berberis purpureocaulis Harber [New Species] IUCN: NE; Evidence: Morphology

HARBER Julian, The Berberis of China and Vietnam, a Revision. Monographs in Systematic Botany from the Missouri Botanical Garden Volume 136: 1-360. 2020 [243] doi: 9781935641186

CHINA (Sichuan*)

Berberis qamdoensis Harber [New Species] IUCN: NE; Evidence: Morphology

HARBER Julian, The Berberis of China and Vietnam, a Revision. Monographs in Systematic Botany from the Missouri Botanical Garden Volume 136: 1-360. 2020 [243] doi: 9781935641186

CHINA (Xizang*)

Berberis qinghaiensis Harber [New Species] IUCN: NE; Evidence: Morphology

HARBER Julian, The Berberis of China and Vietnam, a Revision. Monographs in Systematic Botany from the Missouri Botanical Garden Volume 136: 1-360. 2020 [245] doi: 9781935641186

CHINA (Qinghai*)

Berberis saltuensis Harber [New Species] IUCN: NE; Evidence: Morphology

HARBER Julian, The Berberis of China and Vietnam, a Revision. Monographs in Systematic Botany from the Missouri Botanical Garden Volume 136: 1-360. 2020 [248] doi: 9781935641186

CHINA (Sichuan*)

Berberis saxatilis Harber [New Species] IUCN: NE; Evidence: Morphology

HARBER Julian, The Berberis of China and Vietnam, a Revision. Monographs in Systematic Botany from the Missouri Botanical Garden Volume 136: 1-360. 2020 [249] doi: 9781935641186

CHINA (Yunnan*)

Berberis scrithalis Harber [New Species] IUCN: NE; Evidence: Morphology

HARBER Julian, The Berberis of China and Vietnam, a Revision. Monographs in Systematic Botany from the Missouri Botanical Garden Volume 136: 1-360. 2020 [249] doi: 9781935641186

CHINA (Yunnan*)

Berberis taoensis Harber [New Species] IUCN: NE; Evidence: Morphology

HARBER Julian, The Berberis of China and Vietnam, a Revision. Monographs in Systematic Botany from the Missouri Botanical Garden Volume 136: 1-360. 2020 [257] doi: 9781935641186

CHINA (Gansu*)

Berberis tengchongensis Harber [New Species] IUCN: NE; Evidence: Morphology

HARBER Julian, The Berberis of China and Vietnam, a Revision. Monographs in Systematic Botany from the Missouri Botanical Garden Volume 136: 1-360. 2020 [259] doi: 9781935641186

CHINA (Yunnan*)

Berberis tengii Harber [New Species] IUCN: NE; Evidence: Morphology

HARBER Julian, The Berberis of China and Vietnam, a Revision. Monographs in Systematic Botany from the Missouri Botanical Garden Volume 136: 1-360. 2020 [111] doi: 9781935641186

CHINA (Guizhou*) 
杜诚, 刘军, 叶文, 廖帅, 葛斌杰, 刘冰, 马金双 (2021) 中国植物新分类群、新名称 2020 年度报告. 生物多样性, 29, 10111020. http://www.biodiversity-science.net/CN/10.17520/biods.2021122

Berberis tenuispina Harber [New Species] IUCN: NE; Evidence: Morphology

HARBER Julian, The Berberis of China and Vietnam, a Revision. Monographs in Systematic Botany from the Missouri Botanical Garden Volume 136: 1-360. 2020 [260] doi: 9781935641186

CHINA (Yunnan*)

Berberis tianchiensis Harber [New Species] IUCN: NE; Evidence: Morphology

HARBER Julian, The Berberis of China and Vietnam, a Revision. Monographs in Systematic Botany from the Missouri Botanical Garden Volume 136: 1-360. 2020 [262] doi: 9781935641186

CHINA (Yunnan*)

Berberis wenchuanensis Harber [New Species] IUCN: NE; Evidence: Morphology

HARBER Julian, The Berberis of China and Vietnam, a Revision. Monographs in Systematic Botany from the Missouri Botanical Garden Volume 136: 1-360. 2020 [273] doi: 9781935641186

CHINA (Sichuan*)

Berberis wui Harber [New Species] IUCN: NE; Evidence: Morphology

HARBER Julian, The Berberis of China and Vietnam, a Revision. Monographs in Systematic Botany from the Missouri Botanical Garden Volume 136: 1-360. 2020 [117] doi: 9781935641186

CHINA (Yunnan*)

Berberis xiangchengensis Harber [New Species] IUCN: NE; Evidence: Morphology

HARBER Julian, The Berberis of China and Vietnam, a Revision. Monographs in Systematic Botany from the Missouri Botanical Garden Volume 136: 1-360. 2020 [280] doi: 9781935641186

CHINA (Sichuan*)

Berberis xiaozhongdianensis Harber [New Species] IUCN: NE; Evidence: Morphology

HARBER Julian, The Berberis of China and Vietnam, a Revision. Monographs in Systematic Botany from the Missouri Botanical Garden Volume 136: 1-360. 2020 [280] doi: 9781935641186

CHINA (Yunnan*)

Berberis yaanica Harber [New Species] IUCN: NE; Evidence: Morphology

HARBER Julian, The Berberis of China and Vietnam, a Revision. Monographs in Systematic Botany from the Missouri Botanical Garden Volume 136: 1-360. 2020 [281] doi: 9781935641186

CHINA (Sichuan*)

Berberis yalongensis Harber [New Species] IUCN: NE; Evidence: Morphology

HARBER Julian, The Berberis of China and Vietnam, a Revision. Monographs in Systematic Botany from the Missouri Botanical Garden Volume 136: 1-360. 2020 [281] doi: 9781935641186

CHINA (Sichuan*)

Berberis yanyuanensis Harber [New Species] IUCN: NE; Evidence: Morphology

HARBER Julian, The Berberis of China and Vietnam, a Revision. Monographs in Systematic Botany from the Missouri Botanical Garden Volume 136: 1-360. 2020 [282] doi: 9781935641186

CHINA (Sichuan*)

Berberis yarigongensis Harber [New Species] IUCN: NE; Evidence: Morphology

HARBER Julian, The Berberis of China and Vietnam, a Revision. Monographs in Systematic Botany from the Missouri Botanical Garden Volume 136: 1-360. 2020 [282] doi: 9781935641186

CHINA (Sichuan*)

Berberis yingii Harber [New Species] IUCN: NE; Evidence: Morphology

HARBER Julian, The Berberis of China and Vietnam, a Revision. Monographs in Systematic Botany from the Missouri Botanical Garden Volume 136: 1-360. 2020 [283] doi: 9781935641186

CHINA (Sichuan*)

Berberis yingjunshengii Harber [New Name]

R.: Berberis yingii Harber

HARBER Julian, Berberis yingjunshengii, a New Name for B. yingii (Berberidaceae). Novon 28 (3): 179-179. 2020 [179] doi: 10.3417/2020613

CHINA (Sichuan*)

Berberis yulongshanensis Harber [New Species] IUCN: NE; Evidence: Morphology

HARBER Julian, The Berberis of China and Vietnam, a Revision. Monographs in Systematic Botany from the Missouri Botanical Garden Volume 136: 1-360. 2020 [284] doi: 9781935641186

CHINA (Yunnan*) 
杜诚, 刘军, 叶文, 廖帅, 葛斌杰, 刘冰, 马金双 (2021) 中国植物新分类群、新名称 2020 年度报告. 生物多样性, 29, 10111020. http://www.biodiversity-science.net/CN/10.17520/biods.2021122

Berberis yushuensis Harber [New Species] IUCN: NE; Evidence: Morphology

HARBER Julian, The Berberis of China and Vietnam, a Revision. Monographs in Systematic Botany from the Missouri Botanical Garden Volume 136: 1-360. 2020 [286] doi: 9781935641186

CHINA (Qinghai*)

Berberis zhaoi Harber [New Species] IUCN: NE; Evidence: Morphology

HARBER Julian, The Berberis of China and Vietnam, a Revision. Monographs in Systematic Botany from the Missouri Botanical Garden Volume 136: 1-360. 2020 [287] doi: 9781935641186

CHINA (Yunnan*)

Berberis zhenxiongensis Harber [New Species] IUCN: NE; Evidence: Morphology

HARBER Julian, The Berberis of China and Vietnam, a Revision. Monographs in Systematic Botany from the Missouri Botanical Garden Volume 136: 1-360. 2020 [122] doi: 9781935641186

CHINA (Yunnan*)

Berberis zhongdianensis Harber [New Species] IUCN: NE; Evidence: Morphology

HARBER Julian, The Berberis of China and Vietnam, a Revision. Monographs in Systematic Botany from the Missouri Botanical Garden Volume 136: 1-360. 2020 [288] doi: 9781935641186

CHINA (Yunnan*)

\section{Betulaceae 桦木科}

Carpinus gigabracteatus Z. Qiang Lu 大苞鹅耳枥 [New Species] IUCN: CR; Evidence: Morphology

LU Zhi-qiang, Carpinus gigabracteatus, a New Species from Southeast Yunnan, China. PhytoKeys 145: 47-56. 2020 [50] doi: 10.3897/phytokeys.145.49488

CHINA (Yunnan*)

\section{Blepharostomataceae 睫毛苔科}

Blepharostoma neglecta Vilnet \& Bakalin [New Species] IUCN: NE; Evidence: ITS1-2, trnL-F; Morphology

BAKALIN Vadim A.VILNET Anna A, CHOI Seung Se, NGUYEN Van Sinh, Blepharostoma trichophyllum S.L. (Marchantiophyta):

The Complex of Sibling Species and Hybrids. Plants 9 (1423): 1-26. 2020 [22] doi: 10.3390/plants9111423

CHINA (Sichuan, Yunnan); RUSSIA*

\section{Boraginaceae 紫草科}

Euploca marifolia (J. Koenig ex Retz.) Ancy \& P. Javad [New Combination]

B.: Heliotropium marifolium J. Koenig ex Retz.

ANTONY Anna Ancy, JAVAD P., The Heliotropium marifolium Complex in India and Five New Combinations in Euploca (Heliotropiaceae) from H. sect. Orthostachys (Boraginaceae s.l.). Nordic Journal of Botany 38 (e02913): 1-6. 2020 [2] doi: 10.1111/njb.02913

CHINA (Hainan); INDIA, MALAYA, PAKISTAN, SRI LANKA, THAILAND

Omphalotrigonotis taishunensis Shao Z. Yang, W. W. Pan \& J. P. Zhong 泰顺血果草 [New Species] IUCN: NE; Evidence: Morphology

YANG Shao-zong, PAN Wen-wen, LIU Xi, WANG Jun-feng, SUN Xiao-xia, DING Bing-yang, Omphalotrigonotis taishunensis, a New Species of Boraginaceae from Zhejiang. Journal of Hangzhou Normal University (Natural Science Edition) 19 (3): $258-260$. 2020 [258] doi: 10.12191/j.issn.1674-232X.2020.03.007

CHINA (Zhejiang*)

Onosma fuyunensis Yi He \& Q. R. Liu [New Species] IUCN: LC; Evidence: Morphology

HE Yi, XU Xue-min, ZHOU Yu, LIU Quan-ru, Onosma fuyunensis (Boraginaceae), a New Species from Xinjiang, China. PhytoKeys 144: 11-22. 2020 [13] doi: 10.3897/phytokeys.144.33287

CHINA (Xinjiang*)

Onosma wardii (W. W. Sm.) I. M. Johnst. [New Synonyms]

S.: Onosma mertensioides I. M. Johnst.; Onosma tenuicaulis Riedl; Onosma zayuensis Y. L. Liu; Onosma xiangchengensis W. T. Wang HE Yi, LIU Dan-hui, HUANG Ti-ran, LIU Quan-ru, Taxonomic Notes on Onosma wardii (Boraginaceae) from Southwestern China. Phytotaxa 433 (4): 277-287. 2020 [285] doi: 10.11646/phytotaxa.433.4.3

CHINA (Sichuan, Xizang, Yunnan*)

Trigonotis motuoensis Q. R. Liu \& Xue M. Xu [New Species] IUCN: LC; Evidence: nrITS; Morphology

XU Xue-min, LIU Dan-hui, HE Yi, LIU Quan-ru, Trigonotis motuoensis (Boraginaceae), a New Species from Xizang, China. Phytotaxa 461 (4): 233-242. 2020 [237] doi: 10.11646/phytotaxa.461.4.1 
杜诚, 刘军, 叶文, 廖帅, 葛斌杰, 刘冰, 马金双 (2021) 中国植物新分类群、新名称 2020 年度报告. 生物多样性, 29, 10111020. http://www.biodiversity-science.net/CN/10.17520/biods.2021122

CHINA (Xizang*)

\section{Burmanniaceae 水玉穊科}

Burmannia decurrens Xiao Juan Li \& D. X. Zhang [New Species] IUCN: LC; Evidence: nrDNA; Morphology

LI Xiao-juan, ZHANG Kai, QIAN Xin, WU Ming-song, ZHANG Dian-xiang, Burmannia decurrens (Burmanniaceae), a New Mycoheterotrophic Species from Southwestern Guangdong, China. Nordic Journal of Botany 38 (e02718): 1-6. 2020 [3] doi: 10.1111/njb.02718

CHINA (Guangdong*)

Thismia jianfenglingensis Han Xu, H. J. Yang \& S. Q. Fang 尖峰水玉杯 [New Species] IUCN: NE; Evidence: Morphology

XU Han, YANG Hai-jun, LIN Ming-xian, CORRALES Adriana, HOGAN James Aaron, LI Yi-de, FANG Su-qin, Thismia jianfenglingensis (Thismiaceae), a New Species of Fairy Lantern from Hainan Island, China. Phytotaxa 429 (2): 179-185. 2020 [180] doi: 10.11646/phytotaxa.429.2.9

CHINA (Hainan*)

\section{Buxaceae 黄杨科}

Buxus harlandii Hance 匙叶黄杨 [Correction]

A.: Buxus bodinieri auct. non. H. Lév.: Z. Wei \& Y. B. Zhang in Z. Wei \& Y. Q. He, Fl. Zhejiang 3: 497. 1993

CHEN Feng, XIE Wen-yuan, ZHANG Shui-li, ZHANG Fen-yao, CHEN Zheng-hai, Additional Notes on the Seed Plants in Zhejiang (II). Journal of Hangzhou Normal University (Natural Science Edition) 19 (6): 96-102. 2020 [96] doi: 10.12191/j.issn.1674232X.2020.06.014

CHINA (Zhejiang)

\section{Cactaceae 仙人掌科}

Opuntia cespitosa Raf. 二色仙人掌 [New Record]

LI Xin-hua, ZHOU Wen, GUO Jia-cheng, JIA Shuang, HUANG Si-yu, Opuntia cespitosa Rafinesque, a New Naturalized Species of Cactaceae from China. Journal of Tropical and Subtropical Botany 28 (2): 192-196. 2020 [193] doi: 10.11926/jtsb.4168 CHINA (Jiangsu); UNITED STATES*

\section{Cannabaceae 大麻科}

Cannabis sativa L. var. afghanica (Vavilov) McPartl. \& E. Small [New Combination]

B.: Cannabis sativa L. f. afghanica Vavilov

MCPARTLAND John M., SMALL Ernest, A Classification of Endangered High-THC Cannabis (Cannabis sativa subsp. indica) Domesticates and Their Wild Relatives. PhytoKeys 144: 81-112. 2020 [97] doi: 10.3897/phytokeys.144.46700

CHINA (Xinjiang); AFGHANISTAN*

\section{Caprifoliaceae 忍冬科}

Acanthocalyx delavayi (Franch.) M. J. Cannon [Typification]

MU Qi-yong, YU Chih-chieh, XING Yao-wu, Notes on the Type Specimen of Acanthocalyx delavayi (Caprifoliaceae) at Herbarium of the National Museum of Natural History in Paris (P). Phytotaxa 451 (1): 90-92. 2020 [90] doi: 10.11646/phytotaxa.451.1.9 CHINA (Yunnan*)

Lonicera omissa P. L. Chiu, Z. H. Chen \& Y. L. Xu 无毛忍冬 [New Species] IUCN: NE; Evidence: Morphology

LIU Ju-lian, XU Yue-liang, CHEN Feng, XIE Wen-yuan, CHEN Zheng-hai, A New Species of Lonicera L. from the Southeast of China. Journal of Hangzhou Normal University (Natural Science Edition) 19 (3): 253-257. 2020 [253] doi: 10.12191/j.issn.1674232X.2020.03.006

CHINA (Zhejiang*)

\section{Caryophyllaceae 石竹科}

Pseudostellaria heterantha (Maxim.) Pax 异花孩儿参 [New Synomyms]

S.: Pseudostellaria tianmushanensis G. H. Xia \& G. Y. Li

XIE Wen-yuan, CHEN Feng, ZHANG Fen-yao, XU Shao-qing, CHEN Zheng-hai, Correction and Supplement of the Seed Plant Flora in Zhejiang. Journal of Hangzhou Normal University (Natural Science Edition) 19 (3): 247-252. 2020 [247] doi: 10.12191/j.issn.1674-232X.2020.03.005

CHINA (Zhejiang); JAPAN* 
杜诚, 刘军, 叶文, 廖帅, 葛斌杰, 刘冰, 马金双 (2021) 中国植物新分类群、新名称 2020 年度报告. 生物多样性, 29, 10111020. http://www.biodiversity-science.net/CN/10.17520/biods.2021122

Shivparvatia glanduligera (Edgew.) Pusalkar \& D. K. Singh [Typification]

XUE Bin-e, WANG Long-yuan, YAO Gang, Re-lectotypification of Shivparvatia glanduligera, the Type of the Genus Shivparvatia (Alsineae, Caryophyllaceae). PhytoKeys 166: 129-134. 2020 [133] doi: 10.3897/phytokeys.166.58710

CHINA (Xizang); INDIA, NEPAL

Silene ohwii T. C. Hsu, C. K. Liao \& S. W. Chung [New Name]

R.: Melandrium nubigenum Ohwi

HSU Tian-chuan, LIAO Chun-kuei, CHUNG Shih-wen, HUANG Wei-jie, Silene ohwii (Caryophyllaceae), a Replaced Name for Melandrium nubigenum. Phytotaxa 432 (3): 296-300. 2020 [296] doi: 10.11646/phytotaxa.432.3.7

CHINA (Taiwan*)

Silene ohwii T. C. Hsu, C. K. Liao \& S. W. Chung [Typification]

HSU Tian-chuan, LIAO Chun-kuei, CHUNG Shih-wen, HUANG Wei-jie, Silene ohwii (Caryophyllaceae), a Replaced Name for Melandrium nubigenum. Phytotaxa 432 (3): 296-300. 2020 [296] doi: 10.11646/phytotaxa.432.3.7

CHINA (Taiwan*)

Stellaria amplexicaulis (Hand.-Mazz.) Huan C.Wang \& Feng Yang [New Combination]

B.: Stellaria saxatilis Buch.-Ham. ex D. Don var. amplexicaulis Hand.-Mazz.

YANG Feng, LIU Xiao-lan, LI Yu-ran, Tian ye, WANG Huan-chong, Stellaria procumbens sp. nov. and S. amplexicaulis comb. \& stat. nov. (Caryophyllaceae) from Southwest China. Phytotaxa 435 (2): 192-202. 2020 [197] doi: 10.11646/phytotaxa.435.2.6

CHINA (Sichuan*, Yunnan)

Stellaria amplexicaulis (Hand.-Mazz.) Huan C.Wang \& Feng Yang [Typification]

YANG Feng, LIU Xiao-lan, LI Yu-ran, Tian ye, WANG Huan-chong, Stellaria procumbens sp. nov. and S. amplexicaulis comb. \& stat. nov. (Caryophyllaceae) from Southwest China. Phytotaxa 435 (2): 192-202. 2020 [197] doi: 10.11646/phytotaxa.435.2.6

CHINA (Sichuan*, Yunnan)

Stellaria depressa Schmid [Typification]

WANG Wen-qiao, SU Zhi-wei, MA Zhong-hui, Lectotypification of Five Names in the Genus Stellaria (Caryophyllaceae) in China. PhytoKeys 170: 71-81. 2020 [72] doi: 10.3897/phytokeys.175.59527

CHINA (Xinjiang*)

Stellaria ebracteata Kom. [Typification]

WANG Wen-qiao, SU Zhi-wei, MA Zhong-hui, Lectotypification of Five Names in the Genus Stellaria (Caryophyllaceae) in China. PhytoKeys 170: 71-81. 2020 [75] doi: 10.3897/phytokeys.175.59527

CHINA (Heilongjiang); KOREA*

Stellaria filicaulis Makino [Typification]

WANG Wen-qiao, SU Zhi-wei, MA Zhong-hui, Lectotypification of Five Names in the Genus Stellaria (Caryophyllaceae) in China. PhytoKeys 170: 71-81. 2020 [75] doi: 10.3897/phytokeys.175.59527

CHINA (Hebei, Heilongjiang, Jilin, Liaoning, Nei Mongol, Shanxi); JAPAN*, KOREA

Stellaria multipartita Bo Xu bis \& Meng Li [New Species] IUCN: DD; Evidence: ITS; Morphology

SONG Yan-feng, LI Meng, XU Bo, CHEN Shui-fei, CHEN Lin, XIE Chun-ping, Stellaria multipartita (Caryophyllaceae), a New Species from Chongqing, China. Phytotaxa 442 (3): 196-204. 2020 [198] doi: 10.11646/phytotaxa.442.3.5 CHINA (Chongqing*)

Stellaria pentastyla W. Q. Wang, Han F. Xu \& Z. H. Ma [New Species] IUCN: DD; Evidence: ITS, trnL-F, matK; Morphology

WANG Wen-qiao, XU Han-feng, SHEN Kang-long, SU Zhi-wei, MA Zhong-hui, Stellaria pentastyla (Caryophyllaceae), a New Species from Yunnan (China). Phytotaxa 435 (1): 69-75. 2020 [71] doi: 10.11646/phytotaxa.435.1.9

CHINA (Yunnan*)

Stellaria procumbens Huan C. Wang \& Feng Yang [New Species] IUCN: NE; Evidence: Morphology

YANG Feng, LIU Xiao-lan, LI Yu-ran, Tian ye, WANG Huan-chong, Stellaria procumbens sp. nov. and S. amplexicaulis comb. \& stat. nov. (Caryophyllaceae) from Southwest China. Phytotaxa 435 (2): 192-202. 2020 [195] doi: 10.11646/phytotaxa.435.2.6

CHINA (Sichuan, Yunnan*)

Stellaria pusilla Schmid [Typification]

WANG Wen-qiao, SU Zhi-wei, MA Zhong-hui, Lectotypification of Five Names in the Genus Stellaria (Caryophyllaceae) in China. PhytoKeys 170: 71-81. 2020 [79] doi: 10.3897/phytokeys.175.59527

CHINA (Xizang*)

Stellaria yunnanensis Franch. [Typification] 
杜诚, 刘军, 叶文, 廖帅, 葛斌杰, 刘冰, 马金双 (2021) 中国植物新分类群、新名称 2020 年度报告. 生物多样性, 29, 10111020. http://www.biodiversity-science.net/CN/10.17520/biods.2021122

WANG Wen-qiao, SU Zhi-wei, MA Zhong-hui, Lectotypification of Five Names in the Genus Stellaria (Caryophyllaceae) in China. PhytoKeys 170: 71-81. 2020 [72] doi: 10.3897/phytokeys.175.59527

CHINA (Yunnan*)

\section{Celastraceae 卫矛科}

Parnassia nubicola Wall. ex Royle [New Synonyms]

S.: Parnassia tibetana C. P. Tsien ex T. C. Ku; Parnassia nubicola Wall. subsp. occidentalis Schönbeck-Temesy; Parnassia nubicola Wall. var. nana T. C. Ku

MA Yong-hong, ZHOU Han-lu, SHU Yu-min, Taxonomic Note on Parnassia (Celastraceae): the Identity of P. nubicola. PhytoKeys 154: 103-109. 2020 [105] doi: 10.3897/phytokeys.154.54042

CHINA (Xizang, Yunnan); NEPAL*

Salacia malipoensis X. D. Ma \& J. Y. Shen [New Species] IUCN: NE; Evidence: Morphology

MA Xing-da, WANG Wen-guang, SHI Ji-pu, SHEN Jian-yong, Salacia malipoensis (Celastraceae), a New Species from Yunnan, China. Annales Botanici Fennici 57 (1-3): 109-113. 2020 [110] doi: 10.5735/085.057.0115

CHINA (Yunnan*)

\section{Combretaceae 使君子科}

Combretum densiflorum (Wall. ex Planch. \& Miq.) I. M. Turner [New Combination]

B.: Quisqualis densiflora Wall. ex Planch. \& Miq.

MAURIN Olivier, TURNER Ian M., BOATWRIGHT James S., CHRISTENHUSZ Maarten J. M., New Combinations in Combretaceae subtribe Combretinae from Africa and Asia. Phytotaxa 451 (3): 231-237. 2020 [233] doi: 10.11646/phytotaxa.451.3.6

CHINA (Yunnan); MALAYSIA*

Combretum densiflorum (Wall. ex Planch. \& Miq.) I. M. Turner [Typification]

MAURIN Olivier, TURNER Ian M., BOATWRIGHT James S., CHRISTENHUSZ Maarten J. M., New Combinations in Combretaceae subtribe Combretinae from Africa and Asia. Phytotaxa 451 (3): 231-237. 2020 [233] doi: 10.11646/phytotaxa.451.3.6

CHINA (Yunnan); MALAYSIA*

Quisqualis grandiflora Miq. [Typification]

MAURIN Olivier, TURNER Ian M., BOATWRIGHT James S., CHRISTENHUSZ Maarten J. M., New Combinations in Combretaceae subtribe Combretinae from Africa and Asia. Phytotaxa 451 (3): 231-237. 2020 [233] doi: 10.11646/phytotaxa.451.3.6

CHINA ([unclear])

Quisqualis sinensis Lindl. [Typification]

MAURIN Olivier, TURNER Ian M., BOATWRIGHT James S., CHRISTENHUSZ Maarten J. M., New Combinations in Combretaceae subtribe Combretinae from Africa and Asia. Phytotaxa 451 (3): 231-237. 2020 [233] doi: 10.11646/phytotaxa.451.3.6

CHINA ([unclear])

\section{Commelinaceae 鸭跖草科}

Commelina erecta L. 直立鸭跖草 [New Record]

CHEN Po-hao, CHUNG An-ching, WANG Chih-chiang, YANG Sheng-zehn, Commelina erecta L. (Commelinaceae), a Newly Naturalized Species in Taiwan. Taiwan Journal of Biodiversity 22 (2): 151-158. 2020 [153]

CHINA (Taiwan); [AMERICA]

\section{Cornaceae 山茱英科}

Alangium confertiflorum Y. H. Tan \& H. B. Ding 密花八角枫 [New Species] IUCN: EN: B2ab(i, ii, iii, iv); Evidence: Morphology DING Hong-bo, YANG Bin, WANG Ping-yuan, GAN Zhong-li, YAN Guang, LU Xiao-qiang, TAN Yun-hong, Alangium confertiflorum, a New Species of Alangium sect. Alangium (Cornaceae) from China-Laos Transboundary Region. Taiwania 65 (4): 517-520. 2020 [517] doi: 10.6165/tai.2020.65.517

CHINA (Yunnan*); LAOS

\section{Crassulaceae 景天科}

Phedimus yangshanicus Z. Chao [New Species] IUCN: NE; Evidence: ITS; Morphology

CHAO Zhi, Phedimus yangshanicus (Crassulaceae), a New Species from Limestone Hills in Guangdong, China. Phytotaxa 429 (2): 148-156. 2020 [153] doi: 10.11646/phytotaxa.429.2.5

CHINA (Guangdong*) 
杜诚, 刘军, 叶文, 廖帅, 葛斌杰, 刘冰, 马金双 (2021) 中国植物新分类群、新名称 2020 年度报告. 生物多样性, 29, 10111020. http://www.biodiversity-science.net/CN/10.17520/biods.2021122

Rhodiola humilis (Hook. f. \& Thomson) S. H. Fu var. jialiensis Hao Wang, X. Z. Lan \& H. P. Deng 嘉黎红景天 [New Variety]

WANG Hao, LAN Xiao-zhong, DENG Hong-ping, A New Variety of Rhodiola in Tibet — Rhodiola humilis (HK. f. et Thoms.) S. H. Fu var. jialiensis H. Wang, X. Z. Lan \& H. P. Deng. Acta Botanica Boreali-Occidentalia Sinica 40 (1): 174-180. 2020 [175] doi: 10.7606/j.issn.1000-4025.2020.01.0174

CHINA (Xizang)

Sedum nanlingense Yan Liu \& C. Y. Zou [New Species] IUCN: NE; Evidence: ITS; Morphology

ZOU Chun-yu, MENG Shi-yong, LU Zhao-cen, LIU Yan, Sedum nanlingense (Crassulaceae), a New Species from Guangxi, China. Phytotaxa 447 (3): 176-184. 2020 [183] doi: 10.11646/phytotaxa.447.3.3 CHINA (Guangxi*)

\section{Cucurbitaceae 葫芦科}

Trichosanthes glandulosa G. Q. Zhu, H. Z. Peng \& X. H. Liu 腺栝楼 [New Species] IUCN: NE; Evidence: Morphology ZHU Guang-quan, PENG Hua-zheng, LIU Xin-hong, Trichosanthes glandulosa, a New Species of Trichosanthes L. Journal of Zhejiang Forestry Science and Technology 40 (3): 91-94. 2020 [91] doi: 10.3969/j.issn.1001-3776.2020.04.016 CHINA (Zhejiang*)

\section{Cupressaceae 柏科}

Cupressus $\times$ wangii J. Hoch, Maerki \& Rushforth [New Nothospecies]

H.: Cupressus fallax Franco $\times$ Cupressus funebris Endl.

HOCH J., MAERKI D., About Cupressus jiangeensis N. Zhao. Bulletin of the Cupressus Conservation Project 9 (2): 15-22. 2020 [20] CHINA ([unclear])

Cupressus fallax Franco [New Synonyms]

S.: Cupressus jiangeensis N. Chao; Cupressus chengiana S. Y. Hu var. jiangeensis (N. Chao) Silba; Cupressus chengiana S. Y. Hu subsp. jiangeensis (N. Chao) Silba

HOCH J., MAERKI D., About Cupressus jiangeensis N. Zhao. Bulletin of the Cupressus Conservation Project 9 (2): $15-22.2020$ [18] CHINA (Sichuan)

Cupressus gansuensis Maerki \& J. Hoch [New Species] IUCN: NE; Evidence: RAPD; Morphology

HOCH J., MAERKI D., Taxonomy of the Cypresses of Sichuan and Gansu. Bulletin of the Cupressus Conservation Project 9 (1): 312. $2020[4]$

CHINA (Gansu*)

Juniperus pingii W. C. Cheng [Typification]

YANG Yong, HOCH Jean, Typification of Juniperus pingii W. C. Cheng (Cupressaceae). PhytoKeys 170: 39-43. 2020 [42] doi: 10.3897/phytokeys.170.59775

CHINA (Sichuan*)

\section{Cyatheaceae 杪椤科}

Gymnosphaera bonii (Christ) S. Y. Dong 结脉黑杪椤 [New Combination]

B.: Cyathea bonii Christ

DONG Shi-yong, ZUO Zheng-yu, XIAO Yong, HUANG Ling, PHAM Van The, PHAN Ke Loc, KANG Ming, A Contribution to Gymnosphaera (Cyatheaceae) in Mainland Asia: Two New Species, Reinstatement of Cyathea bonii, and Their Phylogenetic Positions. Journal of Systematics and Evolution 10.1111/jse.12679: 1-12. 2020 [10] doi: 10.1111/jse.12679

CHINA (Fujian, Guangdong, Guangxi, Guizhou, Hainan, Hongkong, Yunnan); VIET NAM*

Gymnosphaera henryi (Baker) S. R. Ghosh [Typification]

DONG Shi-yong, HAQUE A.K.M. Kamrul, RAHMAN Mohammad Sayedur, The True Identity of the Gymnosphaera gigantea (Cyatheaceae) in China. Phytotaxa 449 (1): 15-22. 2020 [18] doi: 10.11646/phytotaxa.449.1.2

CHINA (Guangdong, Guangxi, Hainan, Yunnan*); LAOS, MYANMAR, VIETNAM

Gymnosphaera saxicola S. Y. Dong \& Z. Y. Zuo 岩生黑杪椤 [New Species] IUCN: NE; Evidence: rbcL, rbcL-accD, rbcL-atpB, trnGtrnR, trnL-trnF; Morphology

DONG Shi-yong, ZUO Zheng-yu, XIAO Yong, HUANG Ling, PHAM Van The, PHAN Ke Loc, KANG Ming, a Contribution to Gymnosphaera (Cyatheaceae) in Mainland Asia: Two New Species, Reinstatement of Cyathea bonii, and Their Phylogenetic Positions. Journal of Systematics and Evolution 10.1111/jse.12679: 1-12. 2020 [9] doi: 10.1111/jse.12679

CHINA (Yunnan*)

\section{Cyperaceae 莎草科}


杜诚, 刘军, 叶文, 廖帅, 葛斌杰, 刘冰, 马金双 (2021) 中国植物新分类群、新名称 2020 年度报告. 生物多样性, 29, 10111020. http://www.biodiversity-science.net/CN/10.17520/biods.2021122

Carex austrosibirica Melnikov [New Name]

R.: Elyna filifolia Turcz.

MELNIKOV D. G., KRUPKINA L. I., Transfer of the Kobresia Taxa of the Flora of Russia and Adjacent Countries to the Genus Carex

(Cyperaceae). Novosti Ssistematiki Vysshikh Rastenǐ 51 (1): 125-129. 2020 [127] doi: 10.31111/novitates/2020.51.125

CHINA (Gansu, Hebei, Nei Mongol, Shanxi); RUSSIA

Carex caucasigena Melnikov [New Name]

R.: Elyna schoenoides C. A. Mey

MELNIKOV D. G., KRUPKINA L. I., Transfer of the Kobresia Taxa of the Flora of Russia and Adjacent Countries to the Genus Carex (Cyperaceae). Novosti Ssistematiki Vysshikh Rasteniǔ 51 (1): 125-129. 2020 [127] doi: 10.31111/novitates/2020.51.125

CHINA (Gansu, Qinghai, Sichuan, Xinjiang, Xizang, Yunnan); AFGHANISTAN, BHUTAN, INDIA, KAZAKHSTAN, KYRGYZSTAN, MONGOLIA, NEPAL, PAKISTAN, RUSSIA, TAJIKISTAN, UZBEKISTAN

Carex delavayi Franch. [Typification]

LU Yi-fei, JIN Shui-hu, JIN Xiao-feng, Lectotypification of the Name Carex delavayi (Cyperaceae). Phytotaxa 441 (1): 105-107. 2020

[105] doi: 10.11646/phytotaxa.441.1.11

CHINA (Yunnan*)

Carex iranica Melnikov [New Name]

R.: Kobresia persica Kük. \& Bornm.

MELNIKOV D. G., KRUPKINA L. I., Transfer of the Kobresia Taxa of the Flora of Russia and Adjacent Countries to the

Genus Carex (Cyperaceae). Novosti Ssistematiki Vysshikh Rasteniü 51 (1): 125-129. 2020 [127] doi: 10.31111/novitates/2020.51.125

CHINA (Xizang); INDIA, NEPAL

Carex ledongensis H. B. Yang \& G. D. Liu 乐东薹草 [New Species] IUCN: NE; Evidence: Morphology

YANG Hu-biao, LIU Guo-dao, Carex ledongensis (Cyperaceae), a New Sedge from Hainan Isl. of South China. Phytotaxa 461 (2): 72-78. 2020 [72] doi: 10.11646/phytotaxa.461.2.1

CHINA (Hainan*)

Carex pingleensis Z. C. Lu, Y. F. Lu \& X. F. Jin 平乐薹草 [New Species] IUCN: DD; Evidence: Morphology

LU Zhao-cen, SU Yu-lan, LU Yi-fei, JIN Xiao-feng, Carex pingleensis (Carex sect. Mitratae), a New Species of Cyperaceae from Guangxi, China. Taiwania 65 (3): 391-395. 2020 [391] doi: 10.6165/tai.2020.65.391

CHINA (Guangxi*)

Carex retrofracta Kük. subsp. glabrifolia Y. F. Lu, Hong Wang \& X. F. Jin [New Subspecies]

JIN Xiao-feng, LIU Yong-di, LU Yi-fei, SUN Wen-yan, WANG Hong, Notes on Carex (Cyperaceae) from China (VI): the Identity of Carex retrofracta Kük. (sect. Confertiflorae). Phytotaxa 429 (2): 135-147. 2020 [144] doi: 10.11646/phytotaxa.429.2.4

CHINA (Guangxi, Hunan*)

Carex retrofracta Kük. subsp. retrofracta [New Synonyms]

S.: Carex purpureotincta Ohwi; Carex haematorrhyncha Ohwi \& T. Koyama; Carex purpureotincta Ohwi var. sphaerocarpa Ohwi ex T. Koyama; Carex xiangxiensis Z. P. Wang

JIN Xiao-feng, LIU Yong-di, LU Yi-fei, SUN Wen-yan, WANG Hong, Notes on Carex (Cyperaceae) from China (VI): the Identity of Carex retrofracta Kük. (sect. Confertiflorae). Phytotaxa 429 (2): 135-147. 2020 [142] doi: 10.11646/phytotaxa.429.2.4

CHINA (Anhui, Guizhou, Hubei, Hunan, Sichuan, Taiwan, Zhejiang*)

Carex tsushimensis (Ohwi) Ohwi 对马薹草 [New Record]

ZHANG Xiu-yue, LU Yi-fei, LIU Ju-lian, CHEN Wei-jie, JIN Xiao-feng, ZHENG Zi-hong, Carex tsushimensis (Ohwi) Ohwi, a Newly Recorded Species of Cyperaceae from Zhejiang in East China, with Morphological Comparisons among Related Species. Plant Science Journal 38 (5): 599-603. 2020 [600] doi: 10.11913/PSJ.2095-0837.2020.50599

CHINA (Zhejiang); JAPAN*

\section{Daphniphyllaceae 虎皮楠科}

Daphniphyllum peltatum Yan Liu \& T. Meng 盾叶虎皮楠 [New Species] IUCN: CR: C2a(i);D; Evidence: Morphology MENG Tao, NONG Dong-xin, YUAN Quan, LIU Yan, Daphniphyllum peltatum, a New Species of Daphniphyllaceae from Limestone Areas in Southwestern Guangxi, China. Taiwania 65 (2): 232-236. 2020 [232] doi: 10.6165/tai.2020.65.232 CHINA (Guangxi*)

\section{Dryopteridaceae 鳞毛藓科}

Cyrtomium semifertile Jian X. Li \& Xiao J. Li [New Species] IUCN: NE; Evidence: Morphology

LI Xiao-juan, LI Jian-xiu, Cyrtomium Presl (Dryopteridaceae) - a New Species from China. Bangladesh Journal of Botany 49 (3): 703-708. 2020 [703] 
杜诚, 刘军, 叶文, 廖帅, 葛斌杰, 刘冰, 马金双 (2021) 中国植物新分类群、新名称 2020 年度报告. 生物多样性, 29, 10111020. http://www.biodiversity-science.net/CN/10.17520/biods.2021122

CHINA (Shandong*)

\section{Encalyptaceae 大帽蘚科}

Encalypta papillosa C. Feng, J. Kou \& B. Niu [New Species] IUCN: NE; Evidence: Morphology

KOU Jin, FENG Chao, NIU Ben, XIAO Hong-xing, Encalypta papillosa C. Feng, J. Kou \& B. Niu (Encalyptaceae, Musci), a New Species from Tibet, China. Journal of Bryology 42 (4): 326-332. 2020 [327] doi: 10.1080/03736687.2020.1802184

CHINA (Xizang*)

Encalypta pilifera Funck [New Record]

FENG Chao, KOU Jin, WU Ting-ting, WANG Zi, XIAO Hong-xing, Encalypta pilifera New to China and Range Extension of Two

Rare Species. Herzogia 33 (1): 240-247. 2020 [241] doi: 10.13158/heia.33.1.2020.240

CHINA (Nei Mongol, Xizang); [AFRICA], [ASIA], [EUROPE], [AMERICA]

\section{Ericaceae 杜鹃花科}

Rhododendron mucronatum (Blume) G. Don. f. ruyangense X. J. Zhou 汝阳白杜鹃 [New Form]

ZHOU Xiao-jun, LU Xiao-yu, FU Peng-cheng, A New Form of the Genus Rhododendron L. in Henan, China. Northern Horticlture (9): 79-81. 2020 [79] doi: 10.11937/bfyy.20192657

CHINA (Henan)

Rhododendron pudingense X. Y. Dai, C. H. Yang \& Y. P. Ma 普定杜鹃 [New Species] IUCN: EN: B2ab(iii);D; Evidence: Morphology DAI Xiao-yong, YANG Cheng-hua, YANG Bing, CHEN Pu, MA Yong-peng, A New Species of Rhododendron (Ericaceae) from Guizhou, China. PhytoKeys 146: 53-59. 2020 [54] doi: 10.3897/phytokeys.146.51342

CHINA (Guizhou*)

Rhododendron xishuiense C. H. Yang \& C. D. Yang 习水杜鹃 [New Species] IUCN: NE; Evidence: Morphology

TONG Yi-hua, YANG Cheng-hua, XIA Nian-he, Validation of the Name Rhododendron xishuiense (Ericaceae). Phytotaxa 429 (3): 248-250. 2020 [248] doi: 10.11646/phytotaxa.429.3.7

CHINA (Guizhou*)

Vaccinium bullatum (Dop) Sleumer 泡泡叶越橘 [Typification]

NURALIEV Maxim S., FRITSCH Peter W., BEER Anton S., TONG Yi-hua, AVERYANOV Leonid V., KUZNETSOV Andrey N., KUZNETSOVA Svetlana P., A Revision of Vaccinium bullatum (Ericaceae): Floral Morphology, Distribution and Typification. Phytotaxa 433 (1): 55-66. 2020 [56] doi: 10.11646/phytotaxa.433.1.6

CHINA (Guangxi); VIETNAM*

Vaccinium napoense Y. H. Tong \& N. H. Xia [New Species] IUCN: NE; Evidence: Morphology

TONG Yi-hua, HUANG Yu-song, YE Xue-he, CAI Zhuo-yu, XIA Nian-he, Vaccinium napoense, a New Species of V. sect. Conchophyllum (Ericaceae) from Guangxi, China. Nordic Journal of Botany 38 (e02773): 1-4. 2020 [2] doi: 10.1111/njb.02773

CHINA (Guangxi*)

\section{Euphorbiaceae 大戟科}

Croton capitatus Michx. 头序巴豆 [New Record]

XIA Chang-ying, ZHANG Si-yu, WANG Zhen-hua, LI Qiao, WANG Qin, WU Xue-xue, LI Zhen-yu, YU Sheng-xiang, Croton capitatus (Euphorbiaceae), a Newly Naturalizad Alien Species in China. Plant Quarantine 34 (1): 54-56. 2020 [54] doi: 10.19662/j.cnki.issn1005-2755.2020.01.009

CHINA (Anhui); UNITED STATES*

Euphorbia xianxialingensis F. Y. Zhang, W. Y. Xie \& Z. H. Chen 仙霞岭大戟 [New Species] IUCN: NE; Evidence: Morphology ZHANG Fen-yao, CHEN Feng, XIE Wen-yuan, ZHENG Zhi-xin, MAO Mei-hong, CHEN Zheng-hai, Euphorbia xianxialingensis (Euphorbiaceae), a New Species from Zhejiang. Journal of Zhejiang Forestry Science and Technology 40 (1): 82-85. 2020 [82] doi: 10.3969/j.issn.1001-3776.2020.01.013

CHINA (Zhejiang*)

Mallotus japonicus (L. f.) Müll. Arg. 日本野桐 [New Synomyms]

S.: Mallotus tenuifolius Pax var. subjaponicus Croizat

ZHANG Fen-yao, CHEN Feng, XIE Wen-yuan, CHEN Zheng-hai, New Judgements on Some Species of Euphorbiaceae in Zhejiang. Journal of Zhejiang University (Science Edition) 47 (6): 743-748. 2020 [745] doi: 10.3785/j.issn.1008-9497.2020.06.012

10.3785/j.issn.1008-9497.2020.06.012

10.3785/j.issn.1008-9497.2020.06.012

CHINA (Taiwan, Zhejiang); JAPAN, KOREA 
杜诚, 刘军, 叶文, 廖帅, 葛斌杰, 刘冰, 马金双 (2021) 中国植物新分类群、新名称 2020 年度报告. 生物多样性, 29, 10111020. http://www.biodiversity-science.net/CN/10.17520/biods.2021122

Phyllanthus glaucus Wall. ex Müll. Arg. var. trichocladus P. L. Chiu ex Z. H. Chen 毛枝叶下珠 [New Variety]

ZHANG Fen-yao, CHEN Feng, XIE Wen-yuan, CHEN Zheng-hai, New Judgements on Some Species of Euphorbiaceae in Zhejiang. Journal of Zhejiang University (Science Edition) 47 (6): 743-748. 2020 [747] doi: 10.3785/j.issn.1008-9497.2020.06.012

10.3785/j.issn.1008-9497.2020.06.012

10.3785/j.issn.1008-9497.2020.06.012

CHINA (Zhejiang*)

\section{Fabaceae 豆科}

Astragalus cicer L. 鹰嘴黄耆 [New Record]

LI Ding-nan, ZHANG Shu-mei, LI Hong-bo, GUAN Pei-fu, A Newly Naturalized Species of Astragalus L. in China -- Astragalus cicer L. Journal of Liaoning Normal University (Natural Science Edition) 43 (3): 365-371. 2020 [365] doi: 10.11679/lsxblk2020030368 CHINA (Liaoning); [EUROPE]

Astragalus habaheensis Y. X. Liou [Correction]

A.: Astragalus arcuatus auct. non Kar. \& KiR.: L. R. Xu \& Podlech, Fl. China, 10: 420, 2010; Astragalus subarcuatus auct. non Popov: S. B. Ho, Bull. Bot. Res., Harbin, 3, 1: 47, 1983, S. B. Ho, Fl. Reipubl. Popularis Sin. 42 (1): 280, 1993.

KNYAZEV M. S., Astragalus habaheensis (sect. Erioceras, Fabaceae) in Mongolia and China. Novosti Ssistematiki Vysshikh Rastenǐ 51 (1): 39-42. 2020 [40] doi: 10.31111/novitates/2020.51.39

CHINA (Xinjiang*)

Astragalus shaerqinensis Lei Liu \& Zhi Yong Li [New Species] IUCN: NE; Evidence: Morphology

LIU Lei, LI Zhi-yong, SHI, Wen-gui, Astragalus shaerqinensis (Fabaceae), a New Species from Inner Mongolia, China. Annales Botanici Fennici 57 (1-3): 181-183. 2020 [181] doi: 10.5735/085.057.0125

CHINA (Nei Mongol*)

Brachypterum eriocarpum (F. C. How) Adema \& Sirich. [New Combination]

B.: Derris eriocarpa F. C. How

SIRICHAMORN Yotsawate, ADEMA Frits, Four New Combinations in the Legume Genus Brachypterum. Thai Forest Bulletin 48 (1): 57-60. 2020 [58] doi: 10.20531/tfb.2020.48.1.10

CHINA (Guangxi*); LAOS, VIET NAM

Caesalpinia morsei Dunn [Typification]

LEWIS G. P., New Combinations in Guilandina (Leguminosae: Caesalpinioideae). Kew Bulletin 75 (10): 1-3. 2020 [2] doi: 10.1007/S12225-020-9865-7

CHINA (Guangxi*); VIET NAM

Campylotropis albopubescens (Iokawa \& H. Ohashi) M. Liao \& Bo Xu bis [New Combination]

B.: Campylotropis pinetorum (Kurz) Schindl. var. albopubescens Iokawa \& H. Ohashi

LIAO Min, XU Bo, Campylotropis albopubescens stat. nov. (Leguminosae: Papilionoideae: Desmodieae): the Only Species in the Genus Reproduced via Rootstocks. Phytotaxa 454 (3): 226-230. 2020 [227] doi: 10.11646/phytotaxa.454.3.5

CHINA (Yunnan*)

Flemingia kweichowensis Tang \& F. T. Wang ex Y. T. Wei \& S. K. Lee [New Synonyms]

S.: Flemingia kradungensis Niyomdham

DO Truong Van, GAO Xin-fen, Taxonomic Revision of the Genus Flemingia (Leguminosae) from Indo-Chinese Floristic Region. Phytotaxa 429 (1): 1-38. 2020 [26] doi: 10.11646/phytotaxa.429.1.1

CHINA (Guizhou*, Yunnan); THAILAND

Flemingia nana Roxb. ex W. T. Aiton [New Record]

DO Truong Van, GAO Xin-fen, Taxonomic Revision of the Genus Flemingia (Leguminosae) from Indo-Chinese Floristic Region. Phytotaxa 429 (1): 1-38. 2020 [24] doi: 10.11646/phytotaxa.429.1.1

CHINA (Hainan); INDIA*

Flemingia strobilifera (L.) W. T. Aiton [New Synonyms]

S.: Flemingia tiliacea Niyomdham

DO Truong Van, GAO Xin-fen, Taxonomic Revision of the Genus Flemingia (Leguminosae) from Indo-Chinese Floristic Region. Phytotaxa 429 (1): 1-38. 2020 [19] doi: 10.11646/phytotaxa.429.1.1

CHINA (Yunnan); CAMBODIA, INDIA*, LAOS, MYANMAR, THAILAND, VIET NAM

Flemingia weii T. V. Do \& X. F. Gao [New Species] IUCN: NE; Evidence: Morphology

DO Truong Van, GAO Xin-fen, Taxonomic Revision of the Genus Flemingia (Leguminosae) from Indo-Chinese Floristic Region. Phytotaxa 429 (1): 1-38. 2020 [29] doi: 10.11646/phytotaxa.429.1.1 
杜诚, 刘军, 叶文, 廖帅, 葛斌杰, 刘冰, 马金双 (2021) 中国植物新分类群、新名称 2020 年度报告. 生物多样性, 29, 10111020. http://www.biodiversity-science.net/CN/10.17520/biods.2021122

CHINA (Yunnan*)

Guilandina minax (Hance) G. P. Lewis [New Combination]

B.: Caesalpinia minax Hance

LEWIS G. P., New Combinations in Guilandina (Leguminosae: Caesalpinioideae). Kew Bulletin 75 (10): 1-3. 2020 [2] doi: 10.1007/S12225-020-9865-7

CHINA (Guangxi); VIET NAM

Indigofera yuanjiangensis X. F. Gao \& X. L. Zhao [New Species] IUCN: NE; Evidence: Morphology

ZHAO Xue-li, JIANG Li-sha, GAO Xin-fen, Indigofera yuanjiangensis (Fabaceae: Papilionoideae), a New Species from Yunnan, China. Phytotaxa 455 (3): 235-239. 2020 [235] doi: 10.11646/phytotaxa.455.3.7

CHINA (Yunnan*)

Mucuna guangxiensis K. W. Jiang \& Y. Feng Huang 广西油麻藤 [New Species] IUCN: DD; Evidence: Morphology

JIANG Kai-wen, HUANG Yun-feng, MOURA Tânia M., Mucuna guangxiensis, a New Species of Mucuna subg. Macrocarpa (Leguminosae-Papilionoideae) from China. Phytotaxa 433 (2): 145-152. 2020 [149] doi: 10.11646/phytotaxa.433.2.5

CHINA (Guangxi*)

Oxytropis shennongjiaensis D. G. Zhang, J. T. Chen, T. Deng \& H. Sun 神农架棘豆 [New Species] IUCN: DD; Evidence: ITS, trnLF, psbA-trnH; Morphology

CHEN Jun-tong, ZHANG Dai-gui, LV Zhen-yu, HUANG Xian-han, LIU Peng-ju, YANG Jia-Ning, YANG Jing-yuan, TOJIBAEV Komiljon, DENG Tao, SUN Hang, Oxytropis shennongjiaensis (Fabaceae), a New Species from Hubei, Central China. PhytoKeys 149: 117-128. 2020 [120] doi: 10.3897/phytokeys.149.49533

CHINA (Hubei*)

Phanera apertilobata (Merr. \& F. P. Metcalf) K. W. Jiang [New Combination]

B.: Bauhinia apertilobata Merr. \& F. P. Metcalf

JIANG Kai-wen, New Combinations in the Genus Phanera (Fabaceae: Cercidoideae) of China. The Journal of Japanese Botany 95 (4): 211-213. 2020 [212]

CHINA (Fujian, Jiangxi, Guangdong*, Guangxi)

Phanera cercidifolia (D. X. Zhang) K. W. Jiang [New Combination]

B.: Bauhinia cercidifolia D. X. Zhang

JIANG Kai-wen, New Combinations in the Genus Phanera (Fabaceae: Cercidoideae) of China. The Journal of Japanese Botany 95 (4): 211-213. 2020 [212]

CHINA (Guangxi*)

Phanera delavayi (Franch.) Sinou \& Bruneau [New Combination]

B.: Bauhinia delavayi Franch.

SINOU Carole, CARDINAL-MCTEAGUE Warren, BRUNEAU Anne, Testing Generic Limits in Cercidoideae (Leguminosae): Insights from Plastid and Duplicated Nuclear Gene Sequences. Taxon 69 (1): 67-86. 2020 [80] doi: 10.1002/tax.12207

CHINA (Yunnan)

Phanera esquirolii (Gagnep.) Sinou \& Bruneau [New Combination]

B.: Bauhinia esquirolii Gagnep.

SINOU Carole, CARDINAL-MCTEAGUE Warren, BRUNEAU Anne, Testing Generic Limits in Cercidoideae (Leguminosae): Insights from Plastid and Duplicated Nuclear Gene Sequences. Taxon 69 (1): 67-86. 2020 [80] doi: 10.1002/tax.12207

CHINA (Guizhou, Yunnan)

Phanera hypoglauca (Tang \& F.T.Wang ex T.C.Chen) K. W. Jiang [New Combination]

B.: Bauhinia hypoglauca Tang \& F. T. Wang ex T. C. Chen

JIANG Kai-wen, New Combinations in the Genus Phanera (Fabaceae: Cercidoideae) of China. The Journal of Japanese Botany 95 (4): 211-213. 2020 [212]

CHINA (Yunnan*)

Phanera lingyuenensis (T. C. Chen) K. W. Jiang [New Combination]

B.: Bauhinia lingyuenensis T. C. Chen

JIANG Kai-wen, New Combinations in the Genus Phanera (Fabaceae: Cercidoideae) of China. The Journal of Japanese Botany 95 (4): 211-213. 2020 [212]

CHINA (Guangxi*)

Phanera longistipes (T. C. Chen) K. W. Jiang [New Combination]

B.: Bauhinia longistipes T. C. Chen

JIANG Kai-wen, New Combinations in the Genus Phanera (Fabaceae: Cercidoideae) of China. The Journal of Japanese Botany 95 (4): 211-213. 2020 [212] 
杜诚, 刘军, 叶文, 廖帅, 葛斌杰, 刘冰, 马金双 (2021) 中国植物新分类群、新名称 2020 年度报告. 生物多样性, 29, 10111020. http://www.biodiversity-science.net/CN/10.17520/biods.2021122

\section{CHINA (Yunnan*)}

Phanera venustula (T. C. Chen) K. W. Jiang [New Combination]

B.: Bauhinia venustula T. C. Chen

JIANG Kai-wen, New Combinations in the Genus Phanera (Fabaceae: Cercidoideae) of China. The Journal of Japanese Botany 95 (4): 211-213. 2020 [212]

CHINA (Guangxi*)

Rhynchosia acuminatifolia Makino [Typification]

MISHRA Ajay K., GUPTA Vedhika, WAGH Vjay V., Lectotypification of Rhynchosia acuminatifolia (Leguminosae). Phytotaxa 472 (3): 292-294. 2020 [292] doi: 10.11646/phytotaxa.472.3.8

CHINA (Anhui, Guizhou, Jiangsu, Zhejiang); JAPAN*

\section{Fagaceae 壳斗科}

Cyclobalanopsis reclinatocaulis M. M. Lin 软枝青冈 [New Species] IUCN: VU; Evidence: Morphology

LIN Qin-wen, ZHONG Yi-xin, LIN Mu-mu, Cyclobalanopsis reclinatocaulis, a New Species of Fagaceae from Fujian Province. Bulletin of Botanical Research 40 (1): 10-14. 2020 [11] doi: 10.7525/j.issn.1673-5102.2020.01.003

CHINA (Fujian*)

Quercus baloot Griff. 巴洛特栎 [New Record]

LIU Cheng, YA Ji-dong, GUO Yong-jie, CAI Jie, ZHANG Ting, Newly recorded species of seed plants from Xizang, China. Biodiversity Science 28 (10): 1238-1245. 2020 [1239] doi: 10.17520/biods.2020161

CHINA (Xizang); AFGHANISTAN, INDIA*, PAKISTAN

Quercus dehangensis G. X. Chen, D. G. Zhang \& B. Z. Wang 德夯栋 [New Species] IUCN: CR; Evidence: Morphology

XIANG Xiao-mei, WANG Ben-zhong, ZHANG Ji-yuan, ZHANG Dai-gui, CHEN Gong, Quercus dehangensis: a New Species of Quercus (Fagaceae) from Hunan Province, China. Acta Botanica Boreali-Occidentalia Sinica 40 (10): 1778-1783. 2020 [1781] doi: 10.7606/j.issn.1000-4025.2020.10.1778

CHINA (Hunan*)

\section{Fissidentaceae 凤尾蘚科}

Fissidens gracilifolius Brugg.-Nann. \& Nyholm [New Record]

TANG Qi-Ming, HO Boon-Chuan, WEI Yu-Mei, New National Records and Range Extensions of the Moss Genus Fissidens (Fissidentaceae) from Guangxi, China. Hattoria 11: 41-60. 2020 [43]

CHINA (Guangxi*); JAPAN, [EUROPE]

Fissidens laxitextus Broth. ex Gangulee [New Record]

TANG Qi-Ming, HO Boon-Chuan, WEI Yu-Mei, New National Records and Range Extensions of the Moss Genus Fissidens

(Fissidentaceae) from Guangxi, China. Hattoria 11: 41-60. 2020 [49]

CHINA (Guangxi*); INDIA, JAPAN, NEPAL

Fissidens pseudoclosteri Z. Iwats. \& S. S. Kumar [New Record]

TANG Qi-Ming, HO Boon-Chuan, WEI Yu-Mei, New National Records and Range Extensions of the Moss Genus Fissidens

(Fissidentaceae) from Guangxi, China. Hattoria 11: 41-60. 2020 [52]

CHINA (Guangxi*); INDIA, JAPAN

\section{Funariaceae 葫芦蘚科}

Entosthodon elimbatus W. Z. Ma, Shevock \& S. He 无边梨蒴藓 [New Species] IUCN: DD; Evidence: Morphology

MA Wen-zhang, Shevock James R., HE Si, LIU Yan, Entosthodon elimbatus (Bryophyta, Funariaceae), a New Species from the Subalpine Region in Yunnan and E. physcomitrioides New for Mainland China. Phytotaxa 451 (2): 103-112. 2020 [108] doi: 10.11646/phytotaxa.451.2.1

CHINA (Yunnan *)

\section{Gentianaceae 龙胆科}

Swertia angustifolia Buch.-Ham. ex D. Don [Typification]

HUSAIN Danish, BARIK Saroj Kanta, SRIVASTAVA Abhai K., KATIYAR Pushpendra, SAHOO Dinabandhu, HUSAIN Tariq, AGNIHOTRI Priyanka, Nomenclature and Typification of Swertia angustifolia (Gentianaceae), Its Infraspecific Taxa and Synonyms. Taxon 69 (5): 1085-1091. 2020 [831] doi: 10.1002/tax.12276 
杜诚, 刘军, 叶文, 廖帅, 葛斌杰, 刘冰, 马金双 (2021) 中国植物新分类群、新名称 2020 年度报告. 生物多样性, 29, 10111020. http://www.biodiversity-science.net/CN/10.17520/biods.2021122

CHINA (Fujian, Guangdong, Guangxi, Guizhou, Hubei, Hunan, Jiangxi, Yunnan); BHUTAN, INDIA, MYANMAR, NEPAL*, VIET NAM

Swertia angustifolia Buch.-Ham. ex D. Don var. pulchella (D. Don) Burkill [Typification]

HUSAIN Danish, BARIK Saroj Kanta, SRIVASTAVA Abhai K., KATIYAR Pushpendra, SAHOO Dinabandhu, HUSAIN Tariq, AGNIHOTRI Priyanka, Nomenclature and Typification of Swertia angustifolia (Gentianaceae), Its Infraspecific Taxa and Synonyms. Taxon 69 (5): 1085-1091. 2020 [831] doi: 10.1002/tax.12276

CHINA (Fujian, Guangdong, Guangxi, Guizhou, Hubei, Hunan, Jiangxi, Yunnan); BHUTAN, INDIA, NEPAL*

\section{Gesneriaceae 苦苍苔科}

Aeschynanthus monetaria Dunn 贝叶芒毛芦苔 [Rediscovery]

HU Jun, XIONG Yu-ning, LI Li, LIU Qing, WEN Fang, Rediscovery of Aeschynanthus monetaria (Gesneriaceae) in Southeast Tibet, China after more than 100 Years. Phytotaxa 450 (1): 109-114. 2020 [110] doi: 10.11646/phytotaxa.450.1.9

CHINA (Xizang*); INDIA

Allocheilos maguanensis W. H. Chen \& Y. M. Shui [New Species] IUCN: CR: B1ab(iii)+2ab(iii); Evidence: Morphology

CHEN Wen-hong, GUO Shi-wei, WU Jian-yong, CHEN Li, SHUI Yu-min, Two New Species of Allocheilos (Gesneriaceae) from the Karst Regions in Yunnan, China. PhytoKeys 157: 155-166. 2020 [158] doi: 10.3897/phytokeys.157.32729

CHINA (Yunnan*)

Allocheilos rubroglandulosus W. H. Chen \& Y. M. Shui [New Species] IUCN: CR: B1b(v)+2b(v); Evidence: Morphology

CHEN Wen-hong, GUO Shi-wei, WU Jian-yong, CHEN Li, SHUI Yu-min, Two New Species of Allocheilos (Gesneriaceae) from the Karst Regions in Yunnan, China. PhytoKeys 157: 155-166. 2020 [161] doi: 10.3897/phytokeys.157.32729

CHINA (Yunnan*)

Beccarinda cordifolia (Anthony) B. L. Burtt [New Record]

XU Yuan, CHOUDHARY Ritesh Kumar, HAO Gang, HU Chi-ming, Primula subansirica G. D. Pal Is not a Primula (Primulaceae), But Rather Belongs to Gesneriaceae. Nordic Journal of Botany 38 (e02612): 1-4. 2020 [2] doi: 10.1111/njb.02612

CHINA (Xizang); INDIA, MYANMAR*

Beccarinda cordifolia (Anthony) B. L. Burtt [New Synonyms]

S.: Primula subansirica G. D. Pal

XU Yuan, CHOUDHARY Ritesh Kumar, HAO Gang, HU Chi-ming, Primula subansirica G. D. Pal Is not a Primula (Primulaceae), But Rather Belongs to Gesneriaceae. Nordic Journal of Botany 38 (e02612): 1-4. 2020 [2] doi: 10.1111/njb.02612

CHINA (Xizang); INDIA, MYANMAR*

Boeica arunachalensis D. Borah, R. Kr. Singh, Taram \& A. P. Das [New Species] IUCN: NE; Evidence: Morphology

BORAH Dipankar, SINGH Rajeev Kumar, TARAM Momang, DAS Abhaya Prasad, Boeica arunachalensis (Gesneriaceae), a New Species from Indian Eastern Himalaya and Typification of Five Names in Boeica. Indian Forester 146 (9): 871-874. 2020 [871] doi: 10.36808/if/2020/v146i9/150712

CHINA (Xizang*)

Boeica fulva C. B. Clarke [Typification]

BORAH Dipankar, SINGH Rajeev Kumar, TARAM Momang, DAS Abhaya Prasad, Boeica arunachalensis (Gesneriaceae), a New Species from Indian Eastern Himalaya and Typification of Five Names in Boeica. Indian Forester 146 (9): 871-874. 2020 [872] doi: 10.36808/if/2020/v146i9/150712

CHINA (Xizang); BHUTAN, INDIA*

Boeica porosa C. B. Clarke [Typification]

BORAH Dipankar, SINGH Rajeev Kumar, TARAM Momang, DAS Abhaya Prasad, Boeica arunachalensis (Gesneriaceae), a New Species from Indian Eastern Himalaya and Typification of Five Names in Boeica. Indian Forester 146 (9): 871-874. 2020 [874] doi: 10.36808/if/2020/v146i9/150712

CHINA (Yunnan); INDIA, MYANMAR*, VIET NAM

Deinostigma fasciculatum W. H. Chen \& Y. M. Shui 簇花奇柱菅苔 [New Species] IUCN: CR: C2+a+ii/B2+b+iii; Evidence: Morphology

SHUI Yu-min, WU Jian-yong, YU Zhi-yong, GUO Shi-wei, CHEN Li, WEN Fang, CHEN Wen-hong, Deinostigma fasciculatum, a New Species of Gesneriaceae in Yunnan, China. PhytoKeys 157: 199-206. 2020 [201] doi: 10.3897/phytokeys.157.32683

CHINA (Yunnan*)

Didymocarpus lobulatus F. Wen, Xin Hong \& W. Y. Xie [New Species] IUCN: VU: D2; Evidence: Morphology

XIE Wen-yuan, ZHOU Jia-jun, HONG Xin, WEN Fang, Didymocarpus lobulatus (Gesneriaceae), a New Species from Zhejiang Province, East China. PhytoKeys 157: 145-153. 2020 [147] doi: 10.3897/phytokeys.157.30349

CHINA (Zhejiang*) 
杜诚, 刘军, 叶文, 廖帅, 葛斌杰, 刘冰, 马金双 (2021) 中国植物新分类群、新名称 2020 年度报告. 生物多样性, 29, 10111020. http://www.biodiversity-science.net/CN/10.17520/biods.2021122

Didymocarpus longicalyx G. W. Hu \& Q. F. Wang [New Species] IUCN: NE; Evidence: Morphology

ZHANG Cai-fei, TIAN Jing, PENG Shuai, WANG Jun-jie, WANG Yan, HU Guang-wan, WANG Qing-feng, Didymocarpus longicalyx (Gesneriaceae), a New Species from Southwestern Yunnan, China. Phytotaxa 475 (1): 59-66. 2020 [62] doi: 10.11646/phytotaxa.475.1.6

CHINA (Yunnan*)

Didymocarpus sinoindicus N. S. Prasanna, Lei Cai \& V. Gowda [New Species] IUCN: DD; Evidence: Morphology

PRASANNA N. S., LIU De-tuan, SARYAN P., DUAN Shao-zhong, CAI Lei, GOWDA V., Didymocarpus sinoindicus (Gesneriaceae), a New Species from India and China. Rheedea 30 (1): 135-142. 2020 [136] doi: 10.22244/rheedea.2020.30.01.07

CHINA (Yunnan); INDIA*

Hemiboea guangdongensis (Z. Yu Li) Xiao Qian Li \& X. G. Xiang 广东半蒴芦苔 [New Combination]

B.: Hemiboea henryi C. B. Clarke var. guangdongensis Z. Yu Li

LI Xiao-qian, GUO Zhi-you, LI Yang, ZHOU Peng, CHEN Xu-hui, LI Zhen-yu, XIANG Xiao-guo, Hemiboea guangdongensis comb. \& stat. nov., a Cryptic Species Segregated from H. subcapitata (Gesneriaceae) Based on Morphological and Molecular Data. Nordic Journal of Botany 37 (e02574): 1-7. 2020 [5] doi: 10.1111/njb.02574

CHINA (Guangdong*)

Hemiboea yongfuensis Z. P. Huang \& Y. B. Lu [New Species] IUCN: CR; Evidence: ITS, trnL, trnL-F; Morphology

HUANG Zhang-ping, LI Jia-hui, PAN Bo, QIN Xin-mei, ZHANG Qiang, LU Yong-bin, Hemiboea yongfuensis (Gesneriaceae): A Cryptic and Critically Endangered New Species from North Guangxi, China. Nordic Journal of Botany 38 (e02435): 1-8. 2020 [3] doi: 10.1111/njb.02435

CHINA (Guangxi*)

Henckelia anachoreta (Hance) D. J. Middleton \& Mich. Möller [New Synonyms]

S.: Didymostigma trichathera C. X. Ye \& X. G. Shi

YANG Li-hua, FENG Chen, KANG Ming, WEN Fang, The Taxonomic Identity of Didymostigma trichanthera (Gesneriaceae). PhytoKeys 157: 191-197. 2020 [195] doi: 10.3897/phytokeys.157.32577

CHINA (Guangdong*, Guangxi); LAOS, MYANMAR, THAILAND, VIET NAM

Henckelia oblongifolia (Roxb.) D. J. Middleton \& Mich. Möller 长圆叶汉克苣苔 [New Synomyms]

S.: Lysionotus bijantiae D. Borah \& A. Joe

CAI Lei, BORAH Dipankar, DAO Zhi-ling, WEN Fang, Lysionotus bijantiae Is Identified as a New Synonym of Henckelia oblongifolia (Gesneriaceae). Guihaia 40 (10): 1402-1408. 2020 [1404] doi: 10.11931/guihaia.gxzw201902 016

CHINA (Xizang); BANGLADESH, INDIA, MYANMAR

Henckelia siangensis Taram, D. Borah \& Tag [New Species] IUCN: NE; Evidence: Morphology

TARAM Momang, BORAH Dipankar, TAKU Ojar, TAG Hui, Henckelia siangensis (Gesneriaceae): A Remarkable New Species from Northeast India. PhytoKeys 160: 1-6. 2020 [2] doi: 10.3897/phytokeys.160.54459

CHINA (Xizang*)

Lysionotus coccinus G. W. Hu \& Q. F. Wang 猩红吊石苣苔 [New Species] IUCN: NE; Evidence: Morphology

TIAN Jing, ZHANG Cai-fei, PENG Shuai, WANG Jun-jie, WANG Yan, HU Guang-wan, WANG Qing-feng, Lysionotus coccinus (Gesneriaceae), a New Species from Southwestern Yunnan, China. Nordic Journal of Botany 38 (e02912): 1-6. 2020 [2] doi: 10.1111/njb.02912

CHINA (Yunnan*)

Oreocharis aimodisca Lei Cai, Z. L. Dao \& F. Wen 滇东马铃苣苔 [New Species] IUCN: CR: B2(a); Evidence: Morphology

CAI Lei, HUANG Zhang-jie, WEN Fang, DAO Zhi-ling, Two New Species of Oreocharis (Gesneriaceae) from Karst Regions in Yunnan and Notes on O. tetraptera and O. brachypoda from China. PhytoKeys 162: 1-12. 2020 [3] doi: 10.3897/phytokeys.162.52174 CHINA (Yunnan*)

Oreocharis argentifolia Lei Cai \& Z. L. Dao 银叶马铃芭苔 [New Species] IUCN: NE; Evidence: Morphology

Cai Lei, Dao Zhi-ling, Oreocharis argentifolia (Gesneriaceae), a New Species from the Karst Region in Southeastern Yunnan, China. Nordic Journal of Botany 38 (e02699): 1-7. 2020 [2] doi: 10.1111/njb.02699

CHINA (Yunnan*)

Oreocharis baolianis (Qin W. Lin) Li H. Yang \& M. Kang [New Combination]

B.: Beccarinda baolianis Qin W. Lin

YANG Li-hua, WEN Fang, KONG Hang-hui, SUN Zhi-xia, SU Lan-ying, KANG Ming, Two New Combinations in Oreocharis (Gesneriaceae) Based on Morphological, Molecular and Cytological Evidence. PhytoKeys 157: 43-58. 2020 [54] doi: 10.3897/phytokeys.157.32609

CHINA (Fujian*) 
杜诚, 刘军, 叶文, 廖帅, 葛斌杰, 刘冰, 马金双 (2021) 中国植物新分类群、新名称 2020 年度报告. 生物多样性, 29, 10111020. http://www.biodiversity-science.net/CN/10.17520/biods.2021122

Oreocharis eriocarpa W. H. Chen \& Y. M. Shui [New Species] IUCN: VU: D1+D2; Evidence: ITS, trnL-F; Morphology

CHEN Wen-hong, ZHANG Ya-mei, HE De-ming, LI Yong-liang, SHUI Yu-min, Four New Species of Oreocharis (Gesneriaceae) in Yunnan province, China. PhytoKeys 157: 83-99. 2020 [89] doi: 10.3897/phytokeys.157.32284

CHINA (Yunnan*)

Oreocharis flavovirens Xin Hong [New Species] IUCN: NE; Evidence: Morphology

QIN Wei-hua, DING Dong-dong, LI Zhong-lin, GAO Yun-feng, LI Shu, HONG Xin, Oreocharis flavovirens, a New Species of Gesneriaceae from Southern Gansu Province, China. PhytoKeys 157: 101-112. 2020 [102] doi: 10.3897/phytokeys.157.31732

CHINA (Gansu*)

Oreocharis fulva W. H. Chen \& Y. M. Shui [New Species] IUCN: CR: B1ab(iii)+C2a(ii); Evidence: ITS, trnL-F; Morphology

CHEN Wen-hong, ZHANG Ya-mei, HE De-ming, LI Yong-liang, SHUI Yu-min, Four New Species of Oreocharis (Gesneriaceae) in Yunnan province, China. PhytoKeys 157: 83-99. 2020 [91] doi: 10.3897/phytokeys.157.32284

CHINA (Yunnan*)

Oreocharis guileana (B. L. Burtt) Li H. Yang \& F. Wen [New Combination]

B.: Boeica guileana B. L. Burtt

YANG Li-hua, WEN Fang, KONG Hang-hui, SUN Zhi-xia, SU Lan-ying, KANG Ming, Two New Combinations in Oreocharis (Gesneriaceae) Based on Morphological, Molecular and Cytological Evidence. PhytoKeys 157: 43-58. 2020 [52] doi: 10.3897/phytokeys.157.32609

CHINA (Guangdong, Hong Kong*)

Oreocharis jasminina S. J. Ling, F. Wen \& M. X. Ren 迎春花马铃芭苔 [New Species] IUCN: VU; Evidence: ITS, trnL-F; Morphology

LING Shao-jun, GUAN Shu-ping, WEN Fang, SHUI Yu-min, REN Ming-xun, Oreocharis jasminina (Gesneriaceae), a New Species from Mountain Tops of Hainan Island, South China. PhytoKeys 157: 121-135. 2020 [102] doi: 10.3897/phytokeys.157.50246

CHINA (Hainan*)

Oreocharis lacerata W. H. Chen \& Y. M. Shui [New Species] IUCN: VU: D; Evidence: ITS, trnL-F; Morphology

CHEN Wen-hong, ZHANG Ya-mei, HE De-ming, LI Yong-liang, SHUI Yu-min, Four New Species of Oreocharis (Gesneriaceae) in Yunnan province, China. PhytoKeys 157: 83-99. 2020 [93] doi: 10.3897/phytokeys.157.32284

CHINA (Yunnan*)

Oreocharis longipedicellata Lei Cai \& F. Wen 长梗马铃菅苔 [New Species] IUCN: DD; Evidence: Morphology

CAI Lei, HUANG Zhang-jie, WEN Fang, DAO Zhi-ling, Two New Species of Oreocharis (Gesneriaceae) from Karst Regions in Yunnan and Notes on O. tetraptera and O. brachypoda from China. PhytoKeys 162: 1-12. 2020 [7] doi: 10.3897/phytokeys.162.52174 CHINA (Yunnan*)

Oreocharis wenshanensis W. H. Chen \& Y. M. Shui [New Species] IUCN: CR: D1+D2; Evidence: ITS, trnL-F; Morphology

CHEN Wen-hong, ZHANG Ya-mei, HE De-ming, LI Yong-liang, SHUI Yu-min, Four New Species of Oreocharis (Gesneriaceae) in Yunnan province, China. PhytoKeys 157: 83-99. 2020 [96] doi: 10.3897/phytokeys.157.32284

CHINA (Yunnan*)

Oreocharis wumengensis Lei Cai \& Z. L. Dao 乌蒙马铃芭 [New Species] IUCN: NE; Evidence: Morphology

CAI Lei, LIU Fang-pu, YI Xiang-bo, DAO Zhi-ling, Oreocharis wumengensis, a New Species of Gesneriaceae from northeastern Yunnan, China. PhytoKeys 157: 113-119. 2020 [114] doi: 10.3897/phytokeys.157.33071

CHINA (Yunnan*)

Paraboea brevipedunculata Y. M. Shui \& W. H. Chen [New Species] IUCN: NE; Evidence: Morphology

SHUI Yu-min, GUO Shi-wei, CHEN Li, CHEN Wen-hong, Two New Species of Paraboea (Gesneriaceae) in Caryota Obtusa Forests in Southwest China, with Compound and Simple Dichasia, Respectively. PhytoKeys 157: 207-216. 2020 [212] doi: 10.3897/phytokeys.157.32534

CHINA (Yunnan*)

Paraboea dolomitica Z. Yu Li, X. G. Xiang \& Z. Y. Guo [New Species] IUCN: VU: D1; Evidence: ITS, trnL-F; Morphology

GUO Zhi-you, WU Zhao-wen, XU Wei-bin, LI Zhen-yu, XIANG Xiao-guo, Paraboea dolomitica (Gesneriaceae), a New Species from Guizhou, China. PhytoKeys 153: 37-48. 2020 [41] doi: 10.3897/phytokeys.153.50933

CHINA (Guizhou*)

Paraboea minutiflora D. J. Middleton 微花蛛毛苣苔 [New Recorded]

LU Zhao-cen, LIU En-de, HAN Meng-qi, ZHU Xin-xin, NGUYEN Khang Sinh,XU Wei-bin, Discovery of Paraboea minutiflora (Gesneriaceae) from Southeast Yunnan, China with Supplementary Description. Guihaia 40 (10): 1438-1444. 2020 [1439] doi: 10.11931/guihaia.gxzw201909027 
杜诚, 刘军, 叶文, 廖帅, 葛斌杰, 刘冰, 马金双 (2021) 中国植物新分类群、新名称 2020 年度报告. 生物多样性, 29, 10111020. http://www.biodiversity-science.net/CN/10.17520/biods.2021122

\section{CHINA (Yunnan); VIETNAM*}

Paraboea myriantha Y. M. Shui \& W. H. Chen [New Species] IUCN: NE; Evidence: Morphology

SHUI Yu-min, GUO Shi-wei, CHEN Li, CHEN Wen-hong, Two New Species of Paraboea (Gesneriaceae) in Caryota Obtusa Forests in Southwest China, with Compound and Simple Dichasia, Respectively. PhytoKeys 157: 207-216. 2020 [209] doi: 10.3897/phytokeys.157.32534

CHINA (Yunnan*)

Paraboea nanxiensis Lei Cai \& Gui L. Zhang 南溪蛛毛苣苔 [New Species] IUCN: CR: B2a; Evidence: Morphology

ZHANG Gui-liang, CAI Lei, WANG Yi-zhi, ZHANG Gui-sheng, Paraboea nanxiensis (Gesneriaceae), a New Species from Southeastern Yunnan Province, China. Guihaia 40 (10): 1423-1428. 2020 [1424] doi: 10.11931/guihaia.gxzw202002034

CHINA (Yunnan*)

Petrocodon chishuiensis Z. B. Xin, F. Wen \& S. B. Zhou 赤水石山芦苔 [New Species] IUCN: CR: B2ab(iii); Evidence: Morphology XIN Zi-bing, FU Long-fei, HUANG Zhang-jie, LI Shu, MACIEJEWSKI Stephen, WEN Fang, ZHOU Shou-biao, Petrocodon chishuiensis (Gesneriaceae), a New Species Endemic to Guizhou, China. Taiwania 65 (2): 181-186. 2020 [181] doi: 10.6165/tai.2020.65.181

CHINA (Guizhou*)

Petrocodon ionophyllus F. Wen, S. Li \& B. Pan [New Species] IUCN: EN: D; Evidence: Morphology

LI Shu, PAN Bo, XIN Zi-bing, FU Long-fei, HUANG Zhang-jie, WEN Fang, Petrocodon ionophyllus, a New Species of Gesneriaceae from the Limestone Areas of South China. Rheedea 30 (1): 150-158. 2020 [151] doi: 10.22244/rheedea.2020.30.01.09

CHINA (Guangxi*)

Petrocodon luteoflorus Lei Cai \& F. Wen 小黄花石山苣苔 [New Species] IUCN: LC; Evidence: Morphology

FAN Zhi-wei, CAI Lei, YANG Jia-wen, TANG Sheng-hu, WEN Fang, Petrocodon luteoflorus (Gesneriaceae), a New Species from Karst Region in Guizhou, China. PhytoKeys 157: 167-173. 2020 [169] doi: 10.3897/phytokeys.157.32316

CHINA (Guizhou*)

Petrocodon rubiginosus Y. G. Wei \& R. L. Zhang 锈梗石山苣苔 [New Species] IUCN: EN: B2ab(ii, iii); Evidence: Morphology ZHANG Rui-li, LI Shu, MACIEJEWSKI Stephen, WEI Yi-gang, Petrocodon rubiginosus, a New Species of Gesneriaceae from Guangxi, China. PhytoKeys 157: 175-181. 2020 [176] doi: 10.3897/phytokeys.157.32270

CHINA (Guangxi*)

Petrocodon wenshanensis Xin Hong, W. H. Qin \& F. Wen 文山石山苣苔 [New Species] IUCN: CR: B2ab(iii); Evidence: Morphology

LI Zheng-long, QIN Wei-hua, WEN Fang, HE De-ming, HONG Xin, Petrocodon wenshanensis, a New Species of Gesneriaceae from Southwestern China. PhytoKeys 157: 183-189. 2020 [185] doi: 10.3897/phytokeys.157.39624

CHINA (Guangxi*)

Petrocosmea chiwui M. Q. Han, H. Jiang \& Yan Liu 启无石蝴蝶 [New Species] IUCN: NE; Evidence: Morphology

JIANG Hong, HAN Meng-qi, DONG Zhen, HAN Zhou-dong, LIU Yan, Two New Species of Petrocosmea (Gesneriaceae) from Yunnan, China. Nordic Journal of Botany 38 (e02847): 1-7. 2020 [2] doi: 10.1111/njb.02847

CHINA (Yunnan*)

Petrocosmea nanchuanensis Z. Y. Liu, Z. Yu Li \& Z. J. Qiu [New Species] IUCN: CR: A2c; Evidence: ITS, atpI-atpH, PeCYC1D,matK, trnH-psbA, rps16 intron, trnL-F, trnT-L; Morphology

QIU Zhi-jing, ZHANG Jun, BASKARAN Xavier-Ravi, HU Jin, LI Zhen-yu, LIU Zheng-yu, Petrocosmea nanchuanensis (Gesneriaceae), a New Species from Chongqing, China. PhytoKeys 157: 137-144. 2020 [138] doi: 10.3897/phytokeys.157.33625

CHINA (Chongqing*)

Petrocosmea rotundifolia M. Q. Han, H. Jiang \& Yan Liu 圆叶石蝴蝶 [New Species] IUCN: NE; Evidence: Morphology

JIANG Hong, HAN Meng-qi, DONG Zhen, HAN Zhou-dong, LIU Yan, Two New Species of Petrocosmea (Gesneriaceae) from Yunnan, China. Nordic Journal of Botany 38 (e02847): 1-7. 2020 [2] doi: 10.1111/njb.02847

CHINA (Yunnan*)

Primulina flexusa F. Wen, T. Peng \& B. Pan 曲管报春菅苔 [New Species] IUCN: CR: C1; Evidence: Morphology

PENG Tao, PAN Bo, MACIEJEWSKI Stephen, WEN Fang, Primulina flexusa sp. nov. (Gesneriaceae) from Guizhou Province, China. PhytoKeys 159: 61-69. 2020 [62] doi: 10.3897/phytokeys.159.55386

CHINA (Guizhou*)

Primulina hochiensis (Chang C. Huang \& X. X. Chen) Mich. Möller \& A Webe var. ochroleuc F. Wen, Y. Z. Ge \& Z. B. Xin 黄花河 池报春苣苔 [New Variety] 
杜诚, 刘军, 叶文, 廖帅, 葛斌杰, 刘冰, 马金双 (2021) 中国植物新分类群、新名称 2020 年度报告. 生物多样性, 29, 10111020. http://www.biodiversity-science.net/CN/10.17520/biods.2021122

GE Yu-zhen, XIN Zi-bing, FU Long-fei, CHOU Wei-chuen, HUANG Yi, HUANG Zhang-jie, MACIEJEWSKI Stephen, WEN Fang, Primulina hochiensis var. ochroleuca (Gesneriaceae), a New Variety from a Limestone Area of Guangxi, China, and Errata on Five New Species of Primulina. PhytoKeys 152: 111-120. 2020 [113] doi: 10.3897/phytokeys.152.50968

CHINA (Guangxi*)

Primulina huangjiniana W. B. Liao, Q. Fan \& C. Y. Huang 黄进报春亘苔 [New Species] IUCN: CR: B2a; Evidence: Morphology HUANG Cui-ying, MENG Kai-kai, GUO Jian-qiang, CHEN Fang, LIAO Wen-bo, FAN Qiang, Primulina huangjiniana (Gesneriaceae), a New Species from Guangdong, China. Guihaia 40 (10): 1429-1437. 2020 [1432] doi: 10.11931/guihaia.gxzw202003032

CHINA (Guangdong*)

Primulina inflata Li H. Yang \& M. Z. Xu 粗筒小花苍苔 [New Species] IUCN: CR: B2a,b(iii,v);C2a(i); Evidence: Morphology

XU Mei-zhen, KONG Hang-hui, KANG Ming, YANG Li-hua, A New Species of Primulina (Gesneriaceae) from Danxia landform in Jiangxi, China. Taiwania 65 (2): 163-166. 2020 [163] doi: 10.6165/tai.2020.65.163

CHINA (Guangdong*)

Primulina jiuyishanica Kun Liu bis, D. C. Meng \& Z. B. Xin 九嶷山报春苍苔 [New Species] IUCN: LC; Evidence: Morphology

LIU Kun, MENG De-chang, HUANG Zhang-jie, MACIEJEWSKI Stephen, XIN Zi-bing, Primulina jiuyishanica (Gesneriaceae), a New Species from Hunan, China. PhytoKeys 162: 37-44. 2020 [39] doi: 10.3897/phytokeys.162.53763

CHINA (Guangdong*)

Primulina qintangensis Z. B. Xin, W. C. Chou \& F. Wen 覃塘报春苍苔 [New Species] IUCN: VU: C1; Evidence: Morphology

XIN Zi-bing, HUANG Zhang-jie, CHOU Wei-chuen, HUANG Yi, MENG De-chang, WEN Fang, Primulina qintangensis (Gesneriaceae), a New Species from Limestone Areas of Guangxi, China. Acta Botanica Boreali-Occidentalia Sinica 40 (8): $1424-$ 1427. 2020 [1425] doi: 10.7606/j.issn.1000-4025.2020.08.1424

CHINA (Guangxi*)

Primulina spiradiclioides Z. B. Xin \& F. Wen [New Species] IUCN: NE; Evidence: Morphology

XIN Zi-bing, HUANG Zhang-jie, FU Long-fei, LI Shu, WANG Bing-mou, WEN Fang, Primulina spiradiclioides (Gesneriaceae), a New Species from limestone areas in Guangxi, China. Annales Botanici Fennici 57 (4-6): 245-248. 2020 [245] doi: 10.5735/085.057.0408

CHINA (Guangxi*)

Primulina titan Z. B. Xin, W. C. Chou \& F. Wen [New Species] IUCN: VU: D1; Evidence: Morphology

XIN Zi-bing, FU Long-fei, HUANG Zhang-jie, CHOU Wei-chuen, HUANG Yi, WEN Fang, Primulina titan sp. nov. (Gesneriaceae) from a Limestone Area in Northern Guangxi, China. Journal of Botanical Research 2 (3): 1-4. 2020 [2] doi: 10.30564/jrb.v2i3.1994 CHINA (Guangxi*)

Primulina yulinensis Ying Qin \& Yan Liu 玉林小花苣苔 [New Species] IUCN: NE; Evidence: Morphology

QIN Ying, YE Xiao-xia, XU Wei-bin, LIU Yan, Primulina yulinensis, a New Species of Gesneriaceae from Guangxi, China. Taiwania 65 (1): 5-9. 2020 [5] doi: 10.6165/tai.2020.65.5

CHINA (Guangxi*)

Primulina zixingensis Li H. Yang \& B. Pan [New Species] IUCN: NE; Evidence: Morphology

PAN Bo, XU Mei-zhen, TANG Wen-xiu, YANG Li-hua, Primulina zixingensis (Gesneriaceae), a New Species from Hunan, China. Annales Botanici Fennici 57 (1-3): 55-59. 2020 [55] doi: 10.5735/085.057.0107

CHINA (Hunan*)

Rhynchotechum nirijuliense Taram \& D. Borah [New Species] IUCN: DD; Evidence: Morphology

TARAM M., BORAH D., Rhynchotechum nirijuliense (Gesneriaceae), a New Species from Northeast India. The Gardens' Bulletin Singapore 72 (1): 125-129. 2020 [126] doi: 10.26492/gbs72(1).2020-12

CHINA (Xizang*)

Rhynchotechum parviflorum Blume 小花线柱芭苔 [New Recorded]

HONG Xin, WANG Xiu-ping, WEN Fang, Rhynchotechum parviflorum Blume, a Newly Recorded Species of Gesneriaceae from China. Guihaia 40 (10): 1417-1422. 2020 [1418] doi: 10.11931/guihaia.gxzw202003050

CHINA (Guangdong, Guangxi, Hainan, Hong Kong, Xizang); INDIA, INDONESIA*

\section{Gnetaceae 买麻藤科}

Gnetum chinense Y. Yang, Bing Liu \& S. Z. Zhang [New Species] IUCN: LC; Evidence: ITS, matK, rpoC1, psbB-rps12, trnF-trnV; Morphology

JIN Wei-yin, LIU Bing, ZHANG Shou-zhou, WAN Tao, HOU Chen, YANG Yong, Gnetum chinense, a New Species of Gnetaceae from Southwestern China. PhytoKeys 148: 105-117. 2020 [109] doi: 10.3897/phytokeys.148.48510

CHINA (Yunnan*) 
杜诚, 刘军, 叶文, 廖帅, 葛斌杰, 刘冰, 马金双 (2021) 中国植物新分类群、新名称 2020 年度报告. 生物多样性, 29, 10111020. http://www.biodiversity-science.net/CN/10.17520/biods.2021122

\section{Grimmiaceae 紫膤蘚科}

Grimmia capillata De Not. [New Recorded]

ATIGUL Maola, TIAN Xin-min, AYNUR Turgun, MAMTIMIN Sulayman, Four New Records of Grimmia Hedw. (Grimmiaceae, Musci) in Xinjiang, Northwest China. Acta Botanica Boreali-Occidentalia Sinica 40 (7): 1246-1254. 2020 [1247] doi: 10.7606/j.issn.1000-4025.2020.07.124

CHINA (Xinjiang); [ASIA], [AFRICA], [EUROPE]

\section{Grossulariaceae 茶鹿子科}

Ribes fragrans Pall. 白花茶蔍子 [New Record]

DENG Rui, ZHANG Mei-li, ZHOU Ming, ZHENG Bao-jiang, A Newly Recorded Plant of the Genus Ribes from China. Journal of Nanjing Forestry University (Natural Sciences Edition): 1-5. 2020 [2] doi: 10.3969/j.issn.1000-2006.202004060

CHINA (Nei Mongol); MONGOLIA, RUSSIA

\section{Hydrangeaceae 绣球花科}

Hydrangea chinensis Maxim. var. patentihirsuta Z. H. Chen 展毛中国绣球 [New Variety]

CHEN Feng, XIE Wen-yuan, ZHANG Fen-yao, ZHANG Hong-wei, CHEN Xian-xing, CHEN Zheng-hai, Notes on Seed Plant in Zhejiang Province (V). Journal of Zhejiang Forestry Science and Technology 40 (4): 67-72. 2020 [70] doi: 10.3969/j.issn.10013776.2020.04.012

CHINA (Zhejiang*)

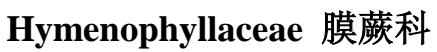

Crepidomanes shenzhenense Hui Wang bis \& X. Yun Wang [New Species] IUCN: EN: C2aii; Evidence: Morphology

WANG Hui, ZHAO Guo-hua, RAVI Baskaran Xavier, LU Shu-gang, Crepidomanes shenzhenense (subg. Crepidomanes; Hymenophyllaceae), a New Filmy Fern Species from Guangdong, Southern China. Phytotaxa 440 (2): 101-107. 2020 [102] doi: 10.11646/phytotaxa.440.2.2

CHINA (Guangdong*)

\section{Iridaceae 盛尾科}

Iris dabashanensis C. A. Wilson [New Species] IUCN: LC; Evidence: matK, trnK, trnL-F; Morphology

WILSON Carol A., Two New Species in Iris series Chinenses (Iridaceae) from South-central China. PhytoKeys 161: 41-60. 2020 [49] doi: $10.3897 /$ phytokeys.161.55483

CHINA (Sichuan*)

Iris probstii C. A. Wilson [New Species] IUCN: VU: D2; Evidence: matK, trnK, trnL-F; Morphology

WILSON Carol A., Two New Species in Iris series Chinenses (Iridaceae) from South-central China. PhytoKeys 161: 41-60. 2020 [53] doi: $10.3897 /$ phytokeys.161.55483

CHINA (Guizhou*)

Iris zhaoana M. B. Crespo, Alexeeva \& Y. E. Xiao [New Name]

R.: Iris potaninii Maxim. var. ionantha Y. T. Zhao

CRESPO Manuel B., ALEXEEVA Nina B., XIAO Yue-e, Iris zhaoana, a New Name for Iris potaninii var. ionantha (I. sect. Pseudoregelia ser. Tigridiae, Iridaceae) from China: Evidence from Morphological and Plastid DNA Data. Phytotaxa 470 (4): $282-$ 289. 2020 [284] doi: 10.11646/phytotaxa.470.4.2

CHINA (Gansu, Qinghai*, Xinjiang, Xizang); MONGOLIA, RUSSIA

\section{Lamiaceae 唇形科}

Caryopteris alternifolia Y. S. Chen \& C. L. Xiang 互叶获 [New Species] IUCN: EN: B2ab, D; Evidence: ITS, ETS; Morphology CHEN You-sheng, XIANG Chun-lei, XU Lian-sheng, JIN Zi-chao, Caryopteris alternifolia (Ajugoideae, Lamiaceae), a Distinct New Species from Guangdong, China. Phytotaxa 461 (1): 62-68. 2020 [64] doi: 10.11646/phytotaxa.461.1.8

CHINA (Guangdong*)

Clinopodium nepalense (Kitam. \& Murata) Bräuchler \& Heub 尼泊尔姜味草 [New Record]

LIU Cheng, YA Ji-dong, GUO Yong-jie, CAI Jie, ZHANG Ting, Newly recorded species of seed plants from Xizang, China. Biodiversity Science 28 (10): 1238-1245. 2020 [1239] doi: 10.17520/biods.2020161

CHINA (Xizang); NEPAL* 
杜诚, 刘军, 叶文, 廖帅, 葛斌杰, 刘冰, 马金双 (2021) 中国植物新分类群、新名称 2020 年度报告. 生物多样性, 29, 10111020. http://www.biodiversity-science.net/CN/10.17520/biods.2021122

Hanceola suffruticosa Y. P. Chen, A. J. Paton \& C. L. Xiang 木茎四轮香 [New Species] IUCN: NE; Evidence: Morphology

CHEN Ya-ping, PATON Alan J., XIANG Chun-lei, Hanceola suffruticosa (Lamiaceae, Nepetoideae), a New Species from the SinoVietnamese Border. PhytoKeys 145: 131-138. 2020 [133] doi: 10.3897/phytokeys.145.49995

CHINA (Yunnan*)

Isodon brevipedunculatus Y. P. Chen \& C. L. Xiang 短梗香茶菜 [New Species] IUCN: NE; Evidence: ITS, ETS; Morphology

CHEN Ya-ping, HUANG Cun-zhong, ZHAO Yue, XIANG Chun-lei, Molecular and Morphological Evidence for a New Species of Isodon (Lamiaceae) from Southern China. Plant Diversity 43 (x): 1/9-9/9. 2020 [5/9] doi: 10.1016/j.pld.2020.06.004

CHINA (Guangdong*, Hunan)

Meehania zheminensis A. Takano, Pan Li \& G. H. Xia 浙闽龙头草 [New Species] IUCN: NE; Evidence: ITS, ETS; Morphology

TAKANO Atsuko, SAKAGUCHI Shota, LI Pan, MATSUO Ayumi, SUYAMA Yoshihisa, XIA Guo-hua, LIU Xi, ISAGI Yuji, A Narrow Endemic or a Species Showing Disjunct Distribution? Studies on Meehania montis-koyae Ohwi (Lamiaceae). Plants 9 (1159): 1-16. 2020 [9] doi: 10.3390/plants9091159

CHINA (Fujian, Zhejiang*)

Paraphlomis kuankuoshuiensis R. B. Zhang, Tan Deng \& C. B. Ma [New Species] IUCN: NT; Evidence: Morphology

ZHANG Ren-bo, DENG Tan, DOU Quan-li, WEI Ruo-xun, HE Lin, MA Chong-bo, ZHAO Sheng, HU Shun, Paraphlomis kuankuoshuiensis (Lamiaceae), a New Species from the Limestone Areas of Northern Guizhou, China. PhytoKeys 139: 13-20. 2020 [15] doi: 10.3897/phytokeys.139.47055

CHINA (Guizhou*)

Premna wui Boufford \& B. M. Barthol. [New Synonyms]

S.: Premna cordiformis Li Bing Zhang \& Yi F. Duan [nom. illeg. superfl.]; Premna wuana Q. Wang [nom. illeg. superfl.]

WANG Ying-hui, WANG Qiang, A Superfluous Name of Premna (Lamiaceae). Phytotaxa 449 (1): 95-96. 2020 [95] doi: 10.11646/phytotaxa.449.1.10

CHINA (Yunnan*)

Scutellaria barbata D. Don [New Synomyms]

S.: Scutellaria taipeiensis T. C. Huang, A. Hsiao \& M. J. Wu

CHAO Chien-ti, HUANG Bing-hong, CHANG Jui-tse, LIAO Pei-chun, Taxonomic Notes on Scutellaria taipeiensis (Lamiaceae) from Morphological and Molecular Data. PhytoKeys 140: 33-45. 2020 [41] doi: 10.3897/phytokeys.140.48578

CHINA (Taiwan); NEPAL

\section{Lauraceae 樟科}

Alseodaphnopsis maguanensis Lang Li \& J. Li [New Species] IUCN: CR: C2a(i); Evidence: ITS, LEAFY intron II; Morphology

LI Lang, TAN Yun-hong, MENG Hong-hu, MA Hui, LI Jie, Two New Species of Alseodaphnopsis (Lauraceae) from Southwestern China and Northern Myanmar: Evidence from Morphological and Molecular Analyses. PhytoKeys 138: 27-39. 2020 [31] doi: 10.3897/phytokeys.138.38569

CHINA (Yunnan*)

Lindera reflexa Hemsl. var. impressivena G. Y. Li \& J. F. Wang 陷脉山橿 [New Variety]

CHEN Zheng-hai, ZHOU Qing, CHEN Gao-kun, XIE Wen-yuan, LI Gen-you, Notes on Seed Plant in Zhejiang Province (VI). Journal of Zhejiang Forestry Science and Technology 40 (4): 73-77. 2020 [75] doi: 10.3969/j.issn.1001-3776.2020.04.013

CHINA (Zhejiang*)

Litsea verticillata Hance [Typification]

KUMAR Anand, Second-step Lectotypification of Six Names of Litsea (Lauraceae). Phytotaxa 460 (3): 214-224. 2020 [225] doi: 10.11646/phytotaxa.460.3.4

CHINA (Guangdong*)

Phoebe hekouensis Bing Liu, W. Y. Jin, L. N. Zhao \& Y. Yang [New Species] IUCN: CR; Evidence: ITS, psbA-trnH; Morphology

LIU Bing, JIN Wei-yin, ZHAO Li-na, YANG Yong, A New Species of Phoebe (Lauraceae) from South-western China. PhytoKeys 140: 101-106. 2020 [102] doi: 10.3897/phytokeys.140.47664

CHINA (Yunnan*)

\section{Lejeuneaceae 细鳞苔科}

Frullania dasyueshanensis Schäf.-Verw. \& G. Winter bis. [New Species] IUCN: NE; Evidence: Morphology

SCHÄFER-VERWIM Alfons, WINTER Gerhard, SCHÄFER-VERWIM Alfons, WINTER Gerhard, Frullania dasyueshanensis, a New Species with Terete Perianth from Taiwan (Marchantiophyta: Frullaniaceae). Frahmia 20: 1-14. 2020 [2]

CHINA (Taiwan*) 
杜诚, 刘军, 叶文, 廖帅, 葛斌杰, 刘冰, 马金双 (2021) 中国植物新分类群、新名称 2020 年度报告. 生物多样性, 29, 10111020. http://www.biodiversity-science.net/CN/10.17520/biods.2021122

Spruceanthus planifolius (Horik.) X. Q. Shi, R. L. Zhu \& Gradst. [New Record]

TU Shu-wen, SHI Rui-ping, TANG Lu-yan, LI Lu-yan, LI Yi-heng, TANG Xia, DAI Zun, XING Shi-chen, SHI Xue-qin, GRADSTEIN S. Robbert, WANG Jian, Floristic Habitat Sampling Yielded Spruceanthus planifolius (Lejeuneaceae, Marchantiophyta) New to China. Phytotaxa 455 (3): 229-234. 2020 [230]

CHINA (Zhejiang); JAPAN, KOREA

\section{Liliaceae 百合科}

Lilium sealyi Y. D. Gao [New Name]

R.: Lilium farreri (W. E. Evans) Y. D. Gao [nom. illeg.]; Nomocharis farreri (W. E. Evans) Harrow; Nomocharis pardanthina Franch. var. farreri W. E. Evans

GAO Yun-dong, GAO Xin-fen, Lilium sealyi Y. D. Gao, A New Name for Lilium farreri (W. E. Evans) Y. D. Gao. Phytotaxa 449 (3): 299-300. 2020 [299] doi: 10.11646/phytotaxa.449.3.9

CHINA (Yunnan); MYANMAR*

Lilium sealyi Y. D. Gao [Typification]

GAO Yun-dong, GAO Xin-fen, Lilium sealyi Y. D. Gao, A New Name for Lilium farreri (W. E. Evans) Y. D. Gao. Phytotaxa 449 (3): 299-300. 2020 [299] doi: 10.11646/phytotaxa.449.3.9

CHINA (Yunnan); MYANMAR*

Lilium tenii H. Lév. [New Synonyms]

S.: Lilium lijiangense L. J. Peng

GAO Yun-dong, GAO Xin-fen, Restore the Name Lilium tenii H. Lév. (Liliaceae), Which Has Priority Over the Later Synonym L. lijiangense L. J. Peng. Phytotaxa 428 (3): 295-300. 2020 [298] doi: 10.11646/phytotaxa.428.3.11

CHINA (Sichuan, Yunnan*)

Notholirion bulbuliferum (Lingelsh.) Stearn [New Synonyms]

S.: Notholirion campanulatum Cotton \& Stearn

LI Juan, ZHOU Song-dong, YANG Mei, XIE Deng-feng, HE Xing-jin, Notholirion campanulatum Is Co-Specific with N. bulbuliferum (Liliaceae) Based on Morphology and Molecular Data. Phytotaxa 471 (3): 234-246. 2020 [284] doi: 10.11646/phytotaxa.471.3.5

CHINA (Shanxi, Sichuan*, Xizang)

Tricyrtis chinensis Hir. Takah. bis var. glandulosa Z. H. Chen, G. Y. Li \& W. Y. Xie 毛果油点草 [New Variety]

CHEN Zheng-hai, ZHOU Qing, CHEN Gao-kun, XIE Wen-yuan, LI Gen-you, Notes on Seed Plant in Zhejiang Province (VI). Journal of Zhejiang Forestry Science and Technology 40 (4): 73-77. 2020 [76] doi: 10.3969/j.issn.1001-3776.2020.04.013

CHINA (Zhejiang*)

\section{Loganiaceae 马钱科}

Mitreola crystallina Y. M. Shui \& W. H. Chen 水晶度量草 [New Species] IUCN: DD; Evidence: Morphology

YOU Jian-rong, RAN Juan, LIU Cheng, SHUI Yu-min, LI Jia-xiang, WU Lei, Validation of the Name Mitreola crystallina (Loganiaceae), a New Species Endemic to Southwestern China. Phytotaxa 471 (2): 139-144. 2020 [140] doi: 10.11646/phytotaxa.471.2.4

CHINA (Yunnan*)

\section{Lythraceae 千屈菜科}

Lagerstroemia limii Merr. f. albiflora G. Y. Li \& Z. H. Chen 白花福建紫薇 [New Form]

CHEN Zheng-hai, ZHOU Qing, CHEN Gao-kun, XIE Wen-yuan, LI Gen-you, Notes on Seed Plant in Zhejiang Province (VI). Journal of Zhejiang Forestry Science and Technology 40 (4): 73-77. 2020 [76] doi: 10.3969/j.issn.1001-3776.2020.04.013

CHINA (Zhejiang*)

Sonneratia $\times$ zhongcairongii Y. S. Wang \& S. H. Shi [New Nothospecies]

H.: Sonneratia alba Griff. $\times$ Sonneratia apetala Buch.-Ham.

ZHONG Cai-rong, LI Dong-lin, ZHANG Ying, Description of a New Natural Sonneratia Hybrid from Hainan Island, China. PhytoKeys 154: 1-9. 2020 [3] doi: 10.3897/phytokeys.154.53223

CHINA (Hainan*)

Trapa dimorphocarpa Z. S. Diao [New Species] IUCN: NE; Evidence: Morphology

DING Bing-yang, JIN Xiao-feng, Taxonomic Notes on Genus Trapa L. (Trapaceae) in China. Guihaia 40 (1): 1-15. 2020 [9] doi: 10.11931/guihaia.gxzw201901002

CHINA (Fujian*)

Trapa dimorphocarpa Z. S. Diao [Typification] 
杜诚, 刘军, 叶文, 廖帅, 葛斌杰, 刘冰, 马金双 (2021) 中国植物新分类群、新名称 2020 年度报告. 生物多样性, 29, 10111020. http://www.biodiversity-science.net/CN/10.17520/biods.2021122

DING Bing-yang, JIN Xiao-feng, Taxonomic Notes on Genus Trapa L. (Trapaceae) in China. Guihaia 40 (1): 1-15. 2020 [9] doi: 10.11931/guihaia.gXzw201901002

CHINA (Fujian*)

Trapa japonica Flerov [Typification]

DING Bing-yang, JIN Xiao-feng, Taxonomic Notes on Genus Trapa L. (Trapaceae) in China. Guihaia 40 (1): 1-15. 2020 [10] doi: 10.11931/guihaia.gxzw201901002

CHINA; JAPAN*

Trapa natans L. 欧菱 [New Synomyms]

S.: Trapa saissanica (Flerow) V. N. Vassil.

DING Bing-yang, JIN Xiao-feng, Taxonomic Notes on Genus Trapa L. (Trapaceae) in China. Guihaia 40 (1): 1-15. 2020 [5] doi: 10.11931/guihaia.gXzw201901002

CHINA (Hebei, Heilongjiang, Hubei, Jiangsu, Jiangxi, Jilin, Liaoning, Shaanxi, Shandong, Xinjiang); JAPAN, KOREA, RUSSIA; [EUROPE]; [AFRICA]

Trapa natans L. var. bispinosa (Roxb.) Makino 菱 [New Synomyms]

S.: Trapa bicornis Osbeck; Trapa chinensis Lour.; Trapa cochinchinensis Lour.; Trapa taiwanensis Nakai; Trapa bicornis Osbeck var. taiwanensis (Nakai) Z. T. Xiong

DING Bing-yang, JIN Xiao-feng, Taxonomic Notes on Genus Trapa L. (Trapaceae) in China. Guihaia 40 (1): 1-15. 2020 [9] doi: 10.11931/guihaia.gxzw201901002

CHINA (Anhui, Fujian, Guangdong, Guangxi, Guizhou, Hainan, Hebei, Heilongjiang, Henan, Hubei, Hunan, Jiangsu, Jiangxi, Jilin, Liaoning, Nei Mongol, Shaanxi, Shandong, Sichuan, Taiwan, Xinjiang, Xizang, Yunnan, Zhejiang); JAPAN, KOREA, RUSSIA, INDONESIA, MALAYSIA, PHILLIPPINES, VIETNAM, LAOS

Trapa natans L. var. bispinosa (Roxb.) Makino 菱 [Typification]

DING Bing-yang, JIN Xiao-feng, Taxonomic Notes on Genus Trapa L. (Trapaceae) in China. Guihaia 40 (1): 1-15. 2020 [9] doi: 10.11931/guihaia.gxzw201901002

CHINA (Anhui, Fujian, Guangdong, Guangxi, Guizhou, Hainan, Hebei, Heilongjiang, Henan, Hubei, Hunan, Jiangsu, Jiangxi, Jilin, Liaoning, Nei Mongol, Shaanxi, Shandong, Sichuan, Taiwan, Xinjiang, Xizang, Yunnan, Zhejiang); JAPAN, KOREA, RUSSIA, INDONESIA, MALAYSIA, PHILLIPPINES, VIETNAM, LAOS

Trapa natans L. var. complana (Z. T. Xiong) B. Y. Ding \& X. F. Jin 格菱 [New Combination]

B.: Trapa pseudoincisa Nakai var. complana Z. T. Xiong

DING Bing-yang, JIN Xiao-feng, Taxonomic Notes on Genus Trapa L. (Trapaceae) in China. Guihaia 40 (1): 1-15. 2020 [13] doi: 10.11931/guihaia.gxzw201901002

CHINA (Anhui, Heilongjiang, Hubei*, Hunan, Jiangsu, Jiangxi, Jilin, Liaoning, Shaanxi, Shandong, Shanghai, Zhejiang); JAPAN, KORAE, RUSSIA

Trapa natans L. var. complana (Z. T. Xiong) B. Y. Ding \& X. F. Jin 格菱 [New Synomyms]

S.: Trapa pseudoincisa Nakai var. aspinta Z. T. Xiong; Trapa pseudoincisa Nakai var. nanchangensis W. H. Wan

DING Bing-yang, JIN Xiao-feng, Taxonomic Notes on Genus Trapa L. (Trapaceae) in China. Guihaia 40 (1): 1-15. 2020 [13] doi: 10.11931/guihaia.gXzw201901002

CHINA (Anhui, Heilongjiang, Hubei*, Hunan, Jiangsu, Jiangxi, Jilin, Liaoning, Shaanxi, Shandong, Shanghai, Zhejiang); JAPAN, KORAE, RUSSIA

Trapa natans L. var. komarovii (Skvortsov) B. Y. Ding \& X. F. Jin 四角菱 [New Combination]

B.: Trapa amurensis Flerow var. komarovii Skvortzov

DING Bing-yang, JIN Xiao-feng, Taxonomic Notes on Genus Trapa L. (Trapaceae) in China. Guihaia 40 (1): 1-15. 2020 [7] doi: 10.11931/guihaia.gxzw201901002

CHINA (Anhui, Fujian, Hainan, Hebei, Hubei, Hunan, Jiangsu, Jiangxi, Jilin, Liaoning, Shanghai, Yunnan, Zhejiang); INDIA, JAPAN, RUSSIA, THAILAND

Trapa natans L. var. komarovii (Skvortsov) B. Y. Ding \& X. F. Jin 四角菱 [New Synomyms]

S.: Trapa acornis Nakano; Trapa quadrispinosa Roxb.; Trapa bicornis Flerow var. quadrispinosa (Roxb.) Z. T. Xiong; Trapa quadrispinosa Roxb. var. yongxiuensis W. H. Wan

DING Bing-yang, JIN Xiao-feng, Taxonomic Notes on Genus Trapa L. (Trapaceae) in China. Guihaia 40 (1): 1-15. 2020 [7] doi: 10.11931/guihaia.gxzw201901002

CHINA (Anhui, Fujian, Hainan, Hebei, Hubei, Hunan, Jiangsu, Jiangxi, Jilin, Liaoning, Shanghai, Yunnan, Zhejiang); INDIA, JAPAN, RUSSIA, THAILAND

Trapa natans L. var. magnicorona (Z. T. Xiong) B. Y. Ding \& X. F. Jin 冠菱 [New Combination]

B.: Trapa japonica Flerow var. magnicorona Z. T. Xiong 
杜诚, 刘军, 叶文, 廖帅, 葛斌杰, 刘冰, 马金双 (2021) 中国植物新分类群、新名称 2020 年度报告. 生物多样性, 29, 10111020. http://www.biodiversity-science.net/CN/10.17520/biods.2021122

DING Bing-yang, JIN Xiao-feng, Taxonomic Notes on Genus Trapa L. (Trapaceae) in China. Guihaia 40 (1): 1-15. 2020 [7] doi: 10.11931/guihaia.gXzw201901002

CHINA (Heilongjiang, Henan, Hubei*, Jiangxi, Jilin, Liaoning); JAPAN, KOREA, RUSSIA

Trapa natans L. var. magnicorona (Z. T. Xiong) B. Y. Ding \& X. F. Jin 冠菱 [New Synomyms]

S.: Trapa japonica Flerow var. longicollum Z. T. Xiong (Z. T. Xiong 443, holotype: WH)

DING Bing-yang, JIN Xiao-feng, Taxonomic Notes on Genus Trapa L. (Trapaceae) in China. Guihaia 40 (1): 1-15. 2020 [7] doi: 10.11931/guihaia.gxzw201901002

CHINA (Heilongjiang, Henan, Hubei*, Jiangxi, Jilin, Liaoning); JAPAN, KOREA, RUSSIA

Trapa natans L. var. quadricaudata (Glück) B. Y. Ding \& X. F. Jin 野菱 [New Combination]

B.: Trapa incisa Siebold \& Zucc. var. quadricaudata Glück

DING Bing-yang, JIN Xiao-feng, Taxonomic Notes on Genus Trapa L. (Trapaceae) in China. Guihaia 40 (1): 1-15. 2020 [12] doi: 10.11931/guihaia.gxzw201901002

CHINA (Anhui, Fujian, Guizhou, Henan, Hubei, Hunan, Jiangsu, Jiangxi, Shanghai, Sichuan*, Yunnan, Zhejiang); JAPAN, VIETNAM

Trapa natans L. var. quadricaudata (Glück) B. Y. Ding \& X. F. Jin 野菱 [New Synomyms]

S.: Trapa maximowiczii Korsh.; Trapa natans L. var. pumila Nakano

DING Bing-yang, JIN Xiao-feng, Taxonomic Notes on Genus Trapa L. (Trapaceae) in China. Guihaia 40 (1): 1-15. 2020 [12] doi: 10.11931/guihaia.gxzw201901002

CHINA (Anhui, Fujian, Guizhou, Henan, Hubei, Hunan, Jiangsu, Jiangxi, Shanghai, Sichuan*, Yunnan, Zhejiang); JAPAN, VIETNAM

\section{Magnoliaceae 木兰科}

Houpoea cathayana (D. L. Fu \& T. B. Chao) Sima \& H. Yu [New Combination]

B.: Magnolia cathayana D. L. Fu \& T. B. Chao

SIMA Yong-kang, YU Hong, MA Hui-fen, HAO Jia-bo, CHEN Shao-yu, LI Shao-wen, FU Yu-pin, New Combinations in Magnoliaceae. Journal of West China Forestry Science 49 (4): 29-40. 2020 [32] doi: 10.16473/j.cnki.xblykx1972.2020.04.005

CHINA (Hunan*)

Houpoea officinalis (Rehder \& E. H. Wilson) N. H. Xia \& C. Y. Wu var. biloba (Rehder \& E. H. Wilson) Sima \& H. Yu [New Combination]

B.: Magnolia officinalis Rehder \& E. H. Wilson var. biloba Rehder \& E. H. Wilson

SIMA Yong-kang, YU Hong, MA Hui-fen, HAO Jia-bo, CHEN Shao-yu, LI Shao-wen, FU Yu-pin, New Combinations in Magnoliaceae. Journal of West China Forestry Science 49 (4): 29-40. 2020 [32] doi: 10.16473/j.cnki.xblykx1972.2020.04.005

CHINA (Jiangxi*)

Lirianthe shangsiensis (Y. W. Law, R. Z. Zhou \& H. F. Chen) Sima \& H. Yu [New Combination]

B.: Magnolia shangsiensis Y. W. Law, R. Z. Zhou \& H. F. Chen

SIMA Yong-kang, YU Hong, MA Hui-fen, HAO Jia-bo, CHEN Shao-yu, LI Shao-wen, FU Yu-pin, New Combinations in Magnoliaceae. Journal of West China Forestry Science 49 (4): 29-40. 2020 [33] doi: 10.16473/j.cnki.xblykx1972.2020.04.005

CHINA (Guangxi*)

Magnolia admirabilis (Y. W. Law \& R. Z. Zhou ex L. Fu, Q. W. Zeng \& X. M. Hu) C. B. Callaghan \& Png 奇异木莲 [New Combination]

B.: Manglietia admirabilis Y. W. Law \& R. Z. Zhou ex L. Fu, Q. W. Zeng \& X. M. Hu

CALLAGHAN Christopher B., PNG Siak K., Twenty-six Additional New Combinations in the Magnolia (Magnoliaceae) of China and Vietnam. PhytoKeys 146: 1-35. 2020 [5] doi: 10.3897/phytokeys.146.52114

CHINA (Yunnan*)

Magnolia albistaminea (Y. W. Law, R. Z. Zhou \& X. S. Qin) C. B. Callaghan \& Png 白荵木莲 [New Combination]

B.: Manglietia albistaminea Y. W. Law, R. Z. Zhou \& X. S. Qin

CALLAGHAN Christopher B., PNG Siak K., Twenty-six Additional New Combinations in the Magnolia (Magnoliaceae) of China and Vietnam. PhytoKeys 146: 1-35. 2020 [6] doi: 10.3897/phytokeys.146.52114

CHINA (Guangdong*)

Magnolia baotaina (D. L. Fu, Qun Zhang \& M. Xu) C. B. Callaghan \& Png 宝台山玉兰 [New Combination]

B.: Yulania baotaina D. L. Fu, Qun Zhang \& M. Xu

CALLAGHAN Christopher B., PNG Siak K., Twenty-six Additional New Combinations in the Magnolia (Magnoliaceae) of China and Vietnam. PhytoKeys 146: 1-35. 2020 [6] doi: 10.3897/phytokeys.146.52114

CHINA (Yunnan*)

Magnolia caloptila (Y. W. Law \& Y. F. Wu) C. B. Callaghan \& Png 美毛含笑 [New Combination]

B.: Michelia caloptila Y. W. Law \& Y. F. Wu 
杜诚, 刘军, 叶文, 廖帅, 葛斌杰, 刘冰, 马金双 (2021) 中国植物新分类群、新名称 2020 年度报告. 生物多样性, 29, 10111020. http://www.biodiversity-science.net/CN/10.17520/biods.2021122

CALLAGHAN Christopher B., PNG Siak K., Twenty-six Additional New Combinations in the Magnolia (Magnoliaceae) of China and Vietnam. PhytoKeys 146: 1-35. 2020 [7] doi: 10.3897/phytokeys.146.52114

CHINA (Jiangxi*)

Magnolia cathcartii (Hook. f. \& Thomson) Noot. [Typification]

KUMAR Anand, BHARATI Kumar Avinash, Typification of the Names Michelia cathcartii and Magnolia gustavii (Magnoliaceae). Phytotaxa 437 (3): 169-173. 2020 [169] doi: 10.11646/phytotaxa.435.2.6

CHINA (Xizang, Yunnan); INDIA*

Magnolia caudata (M. H. Wu, X. H. Wu \& G. Y. Li) C. B. Callaghan \& Png 尾叶含笑 [New Combination]

B.: Michelia caudata M. H. Wu, X. H. Wu \& G. Y. Li

CALLAGHAN Christopher B., PNG Siak K., Twenty-six Additional New Combinations in the Magnolia (Magnoliaceae) of China and Vietnam. PhytoKeys 146: 1-35. 2020 [7] doi: 10.3897/phytokeys.146.52114

CHINA (Zhejiang*)

Magnolia cylindrica E. H. Wilson f. purpurascens (Ya L. Wang \& S. Z. Zhang) G. Y. Li \& Z. H. Chen 紫黄山木兰 [New Combination] B.: Magnolia cylindrica E. H. Wilson var. purpurascens Ya L. Wang \& S. Z. Zhang

CHEN Zheng-hai, ZHOU Qing, CHEN Gao-kun, XIE Wen-yuan, LI Gen-you, Notes on Seed Plant in Zhejiang Province (VI). Journal of Zhejiang Forestry Science and Technology 40 (4): 73-77. 2020 [76] doi: 10.3969/j.issn.1001-3776.2020.04.013

CHINA (Zhejiang*)

Magnolia fallax (Dandy) C. B. Callaghan \& Png 灰线含笑 [New Combination]

B.: Michelia fallax Dandy

CALLAGHAN Christopher B., PNG Siak K., Twenty-six Additional New Combinations in the Magnolia (Magnoliaceae) of China and Vietnam. PhytoKeys 146: 1-35. 2020 [8] doi: 10.3897/phytokeys.146.52114

CHINA (Hunan*)

Magnolia gelida (T. B. Chao, Zhi X. Chen \& D. L. Fu) C. B. Callaghan \& Png 耐冬含笑 [New Combination]

B.: Michelia gelida T. B. Chao, Zhi X. Chen \& D. L. Fu

CALLAGHAN Christopher B., PNG Siak K., Twenty-six Additional New Combinations in the Magnolia (Magnoliaceae) of China and Vietnam. PhytoKeys 146: 1-35. 2020 [10] doi: 10.3897/phytokeys.146.52114

CHINA (Henan*)

Magnolia guangnanica (Da X. Li \& R. Z. Zhou ex X. M. Hu, Q. W. Zeng \& L. Fu) C. B. Callaghan \& Png 广南木莲 [New Combination]

B.: Manglietia guangnanica Da X. Li \& R. Z. Zhou ex X. M. Hu, Q. W. Zeng \& L. Fu

CALLAGHAN Christopher B., PNG Siak K., Twenty-six Additional New Combinations in the Magnolia (Magnoliaceae) of China and Vietnam. PhytoKeys 146: 1-35. 2020 [10] doi: 10.3897/phytokeys.146.52114

CHINA (Yunnan*)

Magnolia hunanensis (C. L. Peng \& L. H. Yan) C. B. Callaghan \& Png 湖南含笑 [New Combination]

B.: Michelia hunanensis C. L. Peng \& L. H. Yan

CALLAGHAN Christopher B., PNG Siak K., Twenty-six Additional New Combinations in the Magnolia (Magnoliaceae) of China and Vietnam. PhytoKeys 146: 1-35. 2020 [11] doi: 10.3897/phytokeys.146.52114 CHINA (Hunan*)

Magnolia jinggangshanensis (R. L. Liu \& Z. X. Zhang) C. B. Callaghan \& Png 井冈山木莲 [New Combination] B.: Manglietia jinggangshanensis R. L. Liu \& Z. X. Zhang

CALLAGHAN Christopher B., PNG Siak K., Twenty-six Additional New Combinations in the Magnolia (Magnoliaceae) of China and Vietnam. PhytoKeys 146: 1-35. 2020 [12] doi: 10.3897/phytokeys.146.52114

CHINA (Jiangxi*)

Magnolia maguanica (Hung T. Chang \& B. L. Chen) C. B. Callaghan \& Png 马关木莲 [New Combination]

B.: Manglietia maguanica Hung T. Chang \& B. L. Chen

CALLAGHAN Christopher B., PNG Siak K., Twenty-six Additional New Combinations in the Magnolia (Magnoliaceae) of China and Vietnam. PhytoKeys 146: 1-35. 2020 [13] doi: 10.3897/phytokeys.146.52114 CHINA (Yunnan*)

Magnolia maudiae (Dunn) Figlar var. rubicunda (T. P. Yi \& J. C. Fan) C. B. Callaghan \& Png 红花深山含笑 [New Combination] B.: Michelia maudiae Dunn var. rubricunda T. P. Yi \& J. C. Fan

CALLAGHAN Christopher B., PNG Siak K., Twenty-six Additional New Combinations in the Magnolia (Magnoliaceae) of China and Vietnam. PhytoKeys 146: 1-35. 2020 [13] doi: 10.3897/phytokeys.146.52114 CHINA (Sichuan*) 
杜诚, 刘军, 叶文, 廖帅, 葛斌杰, 刘冰, 马金双 (2021) 中国植物新分类群、新名称 2020 年度报告. 生物多样性, 29, 10111020. http://www.biodiversity-science.net/CN/10.17520/biods.2021122

Magnolia multitepala (R. Z. Zhou \& S. G. Jian) C. B. Callaghan \& Png 多瓣含笑 [New Combination]

B.: Michelia multitepala R. Z. Zhou \& S. G. Jian

CALLAGHAN Christopher B., PNG Siak K., Twenty-six Additional New Combinations in the Magnolia (Magnoliaceae) of China and Vietnam. PhytoKeys 146: 1-35. 2020 [14] doi: 10.3897/phytokeys.146.52114

CHINA (Yunnan*)

Magnolia officinalis Rehder \& E. H. Wilson f. rubicunda (T. P. Yi) G. Y. Li \& Z. H. Chen 红花凹叶厚朴 [New Combination] B.: Magnolia officinalis Rehder \& E. H. Wilson var. rubicunda T. P. Yi

CHEN Zheng-hai, ZHOU Qing, CHEN Gao-kun, XIE Wen-yuan, LI Gen-you, Notes on Seed Plant in Zhejiang Province (VI). Journal of Zhejiang Forestry Science and Technology 40 (4): 73-77. 2020 [76] doi: 10.3969/j.issn.1001-3776.2020.04.013

CHINA (Sichuan*)

Magnolia pendula (D. L. Fu) C. B. Callaghan \& Png 垂枝玉兰 [New Combination]

B.: Yulania pendula D. L. Fu

CALLAGHAN Christopher B., PNG Siak K., Twenty-six Additional New Combinations in the Magnolia (Magnoliaceae) of China and Vietnam. PhytoKeys 146: 1-35. 2020 [14] doi: 10.3897/phytokeys.146.52114

CHINA (Sichuan*)

Magnolia pilocarpa Z. Z. Zhao \& Z. W. Xie var. ellipticifolia (D. L. Fu, T. B. Chao \& J. Zhao) C. B. Callaghan \& Png 椭圆叶罗田 玉兰 [New Combination]

B.: Yulania pilocarpa (Z. Z. Zhao \& Z. W. Xie) D. L. Fu var. ellipticifolia D. L. Fu, T. B. Chao \& J. Zhao

CALLAGHAN Christopher B., PNG Siak K., Twenty-six Additional New Combinations in the Magnolia (Magnoliaceae) of China and Vietnam. PhytoKeys 146: 1-35. 2020 [15] doi: 10.3897/phytokeys.146.52114

CHINA (Henan*)

Magnolia platypetala (Hand.-Mazz.) C. B. Callaghan \& Png 阔瓣含笑 [New Combination]

B.: Michelia platypetala Hand.-Mazz.

CALLAGHAN Christopher B., PNG Siak K., Twenty-six Additional New Combinations in the Magnolia (Magnoliaceae) of China and Vietnam. PhytoKeys 146: 1-35. 2020 [16] doi: 10.3897/phytokeys.146.52114

CHINA (Hunan*)

Magnolia puberula (D. L. Fu) C. B. Callaghan \& Png 短毛玉兰 [New Combination]

B.: Yulania puberula D. L. Fu

CALLAGHAN Christopher B., PNG Siak K., Twenty-six Additional New Combinations in the Magnolia (Magnoliaceae) of China and Vietnam. PhytoKeys 146: 1-35. 2020 [17] doi: 10.3897/phytokeys.146.52114

CHINA (Hubei*)

Magnolia pubipedunculata (Q. W. Zeng \& X. M. Hu) C. B. Callaghan \& Png 柔毛花梗木莲 [New Combination]

B.: Manglietia pubipedunculata Q. W. Zeng \& X. M. Hu

CALLAGHAN Christopher B., PNG Siak K., Twenty-six Additional New Combinations in the Magnolia (Magnoliaceae) of China and Vietnam. PhytoKeys 146: 1-35. 2020 [18] doi: 10.3897/phytokeys.146.52114

CHINA (Yunnan*)

Magnolia pubipetala (Q. W. Zeng) C. B. Callaghan \& Png 毛瓣木莲 [New Combination]

B.: Manglietia pubipetala Q. W. Zeng

CALLAGHAN Christopher B., PNG Siak K., Twenty-six Additional New Combinations in the Magnolia (Magnoliaceae) of China and Vietnam. PhytoKeys 146: 1-35. 2020 [19] doi: 10.3897/phytokeys.146.52114

CHINA (Yunnan*)

Magnolia rubriflora (Y. W. Law \& R. Z. Zhou ex F. G. Wang, Q. W. Zeng, R. Z. Zhou \& F. W. Xing) C. B. Callaghan \& Png 红花含 笑 [New Combination]

B.: Michelia rubriflora Y. W. Law \& R. Z. Zhou ex F. G. Wang, Q. W. Zeng, R. Z. Zhou \& F. W. Xing

CALLAGHAN Christopher B., PNG Siak K., Twenty-six Additional New Combinations in the Magnolia (Magnoliaceae) of China and Vietnam. PhytoKeys 146: 1-35. 2020 [19] doi: 10.3897/phytokeys.146.52114

CHINA (Hainan*)

Magnolia rufisyncarpa (Y. W. Law, R. Z. Zhou \& F. G. Wang) C. B. Callaghan \& Png 红雌荵木莲 [New Combination]

B.: Manglietia rufisyncarpa Y. W. Law, R. Z. Zhou \& F. G. Wang

CALLAGHAN Christopher B., PNG Siak K., Twenty-six Additional New Combinations in the Magnolia (Magnoliaceae) of China and Vietnam. PhytoKeys 146: 1-35. 2020 [21] doi: 10.3897/phytokeys.146.52114

CHINA (Yunnan*)

Magnolia septipetala (Z. L. Nong) C. B. Callaghan \& Png 七瓣含笑 [New Combination] 
杜诚, 刘军, 叶文, 廖帅, 葛斌杰, 刘冰, 马金双 (2021) 中国植物新分类群、新名称 2020 年度报告. 生物多样性, 29, 10111020. http://www.biodiversity-science.net/CN/10.17520/biods.2021122

B.: Michelia septipetala Z. L. Nong

CALLAGHAN Christopher B., PNG Siak K., Twenty-six Additional New Combinations in the Magnolia (Magnoliaceae) of China and Vietnam. PhytoKeys 146: 1-35. 2020 [22] doi: 10.3897/phytokeys.146.52114

CHINA (Jiangxi*)

Magnolia sinoconifera (F. N. Wei) C. B. Callaghan \& Png 那坡木莲 [New Combination]

B.: Manglietia sinoconifera F. N. Wei

CALLAGHAN Christopher B., PNG Siak K., Twenty-six Additional New Combinations in the Magnolia (Magnoliaceae) of China and Vietnam. PhytoKeys 146: 1-35. 2020 [24] doi: 10.3897/phytokeys.146.52114

CHINA (Guangxi*)

Magnolia urceolata (D. L. Fu, B. H. Xiong \& X. Chen) C. B. Callaghan \& Png 宽瓣玉兰 [New Combination]

B.: Yulania urceolata D. L. Fu, B. H. Xiong \& X. Chen

CALLAGHAN Christopher B., PNG Siak K., Twenty-six Additional New Combinations in the Magnolia (Magnoliaceae) of China and Vietnam. PhytoKeys 146: 1-35. 2020 [25] doi: 10.3897/phytokeys.146.52114

CHINA (Guizhou*)

Magnolia xinningia (Y. W. Law \& R. Z. Zhou ex Q. X. Ma, Q. W. Zeng, R. Z. Zhou \& F. W. Xing) C. B. Callaghan \& Png 新宁含笑 [New Combination]

B.: Michelia xinningia Y. W. Law \& R. Z. Zhou ex Q. X. Ma, Q. W. Zeng, R. Z. Zhou \& F. W. Xing

CALLAGHAN Christopher B., PNG Siak K., Twenty-six Additional New Combinations in the Magnolia (Magnoliaceae) of China and Vietnam. PhytoKeys 146: 1-35. 2020 [25] doi: 10.3897/phytokeys.146.52114

CHINA (Hunan*)

Yulania biondii (Pamp.) D. L. Fu var. purpurascens (Ya L. Wang \& S. Z. Zhang) Sima \& H. Yu [New Combination]

B.: Magnolia biondii Pamp. var. purpurascens Ya L. Wang \& S. Z. Zhang

SIMA Yong-kang, YU Hong, MA Hui-fen, HAO Jia-bo, CHEN Shao-yu, LI Shao-wen, FU Yu-pin, New Combinations in Magnoliaceae. Journal of West China Forestry Science 49 (4): 29-40. 2020 [39] doi: 10.16473/j.cnki.xblykx1972.2020.04.005

CHINA (Shaanxi*)

Yulania cylindrica (E. H. Wilson) D. L. Fu var. purpurascens (Ya L. Wang \& S. Z. Zhang) Sima \& H. Yu [New Combination] B.: Magnolia cylindrica E. H. Wilson var. purpurascens Ya L. Wang \& S. Z. Zhang

SIMA Yong-kang, YU Hong, MA Hui-fen, HAO Jia-bo, CHEN Shao-yu, LI Shao-wen, FU Yu-pin, New Combinations in Magnoliaceae. Journal of West China Forestry Science 49 (4): 29-40. 2020 [39] doi: 10.16473/j.cnki.xblykx1972.2020.04.005

CHINA (Zhejiang*)

Yulania denudata (Desr.) D. L. Fu var. glabrata (Ya L. Wang \& S. Z. Zhang) Sima \& H. Yu [New Combination]

B.: Magnolia denudata Desr. var. glabrata Ya L. Wang \& S. Z. Zhang

SIMA Yong-kang, YU Hong, MA Hui-fen, HAO Jia-bo, CHEN Shao-yu, LI Shao-wen, FU Yu-pin, New Combinations in Magnoliaceae. Journal of West China Forestry Science 49 (4): 29-40. 2020 [39] doi: 10.16473/j.cnki.xblykx1972.2020.04.005 CHINA (Hunan*)

\section{Malvaceae 锦葵科}

Firmiana daweishanensis Gui L. Zhang \& J. Y. Xiang [New Species] IUCN: EN: B; Evidence: Morphology

ZHANG Gui-liang, CAI Lei, DUAN Jie-qiu, WANG Ting, XIANG Jian-ying, Firmiana daweishanensis sp. nov. (Malvaceae) from Southeast Yunnan, China. Phytotaxa 456 (2): 215-218. 2020 [215] doi: 10.11646/phytotaxa.456.2.10

CHINA (Yunnan*)

Thespesia populnea (L.) Sol. ex Corrêa [Typification]

ARECES-BERAZAIN Fabiola, ACKERMAN James D., A Revision of Thespesia and allied genera in Tribe Gossypieae (MalvaceaeMalvoideae). Brittonia 72 (1): 62-110. 2020 [94] doi: 10.1186/s40529-020-00298-y

CHINA (Guangdong, Hainan, Hong Kong, Taiwan); CAMBODIA, INDIA, JAPAN, PHILIPPINES, SRI LANKA*, THAILAND, VIETNAM; [AFRICA]; [AMERICA]

Tilia oblongifolia Rehder 矩圆叶椴 [New Synomyms]

S.: Tilia tuan Szyszył. subsp. oblongifolia (Rehder) Pigott

ZHANG Zhong-zhao, CHEN Zheng-hai, CHEN Feng, XIE Wen-yuan, LI Gen-you, Additional Notes on the Seed Plant Flora of Zhejiang( VII ). Journal of Zhejiang Forestry Science and Technology 40 (6): 52-55. 2020 [53] doi: 10.3969/j.issn.10013776.2020.06.008

CHINA (Anhui*, Hunan, Zhejiang)

\section{Marattiaceae 合囊鄀科}


杜诚, 刘军, 叶文, 廖帅, 葛斌杰, 刘冰, 马金双 (2021) 中国植物新分类群、新名称 2020 年度报告. 生物多样性, 29, 10111020. http://www.biodiversity-science.net/CN/10.17520/biods.2021122

Angiopteris tamdaoensis (Hayata) J. Y. Xiang \& Ting Wang tris [New Combination]

B.: Archangiopteris tamdaoensis Hayata

WANG Ting, XIAO Bo, LIU En-de, NGUYEN Khang Sinh, DUAN Jie-qiu, WANG Kang-lin, YAN Yue-hong, XIANG Jian-ying, Rediscovery of Angiopteris tonkinensis (Marattiaceae) after 100 Years, and Its Revision. PhytoKeys 161: 1-9. 2020 [7] doi: 10.3897/phytokeys.161.54912

CHINA (Hainan); VIET NAM

Angiopteris tonkinensis (Hayata) J. M. Camus [New Record]

WANG Ting, XIAO Bo, LIU En-de, NGUYEN Khang Sinh, DUAN Jie-qiu, WANG Kang-lin, YAN Yue-hong, XIANG Jian-ying, Rediscovery of Angiopteris tonkinensis (Marattiaceae) after 100 Years, and Its Revision. PhytoKeys 161: 1-9. 2020 [7] doi: 10.3897/phytokeys.161.54912

CHINA (Yunnan); VIET NAM*

\section{Mayacaceae 花水蘚科}

Mayaca fluviatilis Aubl. [New Record]

SU Fan, GUO Ya-nan, ZHOU Xin-xin, WANG Rui-jiang, Mayacaceae, a Newly Naturalized Family for the Flora of China. Phytotaxa 447 (1): 77-80. 2020 [77] doi: 10.11646/phytotaxa.447.1.9

CHINA (Guangdong); FRENCH GUIANA*

\section{Melanthiaceae 䔉芦科}

Chamaelirium shimentaiense Y. H. Tong, C. M. He \& Y. Q. Li 石门台白丝草 [New Species] IUCN: VU: D2; Evidence: Morphology TONG Yi-hua, LI Yuan-qiu, GUO Guo-xin, DAI Ke-yuan, ZENG Yang-jin, HE Chun-mei, Chamaelirium shimentaiense (Melanthiaceae), a New Species from Guangdong, China. Nordic Journal of Botany 38 (e02634): 1-3. 2020 [2] doi: 10.1111/njb.02634

CHINA (Guangdong*)

\section{Melastomataceae 野牡丹科}

Anplectrum yunnanense Kraenzl. [Typification]

KARTONEGORO Abdulrokhman, LIU Ying, OLIVEIRA de Sylvia Mota, WELZEN Peter Van, A Taxonomic Revision of Pseudodissochaeta (Melastomataceae, Dissochaeteae). Phytotaxa 468 (2): 159-174. 2020 [167] doi: 10.11646/phytotaxa.468.2.1 CHINA (Yunnan*)

Bredia hispida J. H. Dai \& Ying Liu [New Species] IUCN: NE; Evidence: nrITS; Morphology

DAI Jin-hong, ZHOU Qiu-jie, ZHOU Ren-chao, LIU Ying, A New Species of Bredia (Sonerileae, Melastomataceae) from Sichuan, China. PhytoKeys 152: 1-14. 2020 [9] doi: 10.3897/phytokeys.152.53512

CHINA (Sichuan*)

Bredia reniformis C. M. He, Y. H. Tong \& S. Jin Zeng [New Species] IUCN: DD; Evidence: Morphology

HE Chun-mei, ZENG Si-jin, LI Shu-wan, TONG Yi-hua, Bredia reniformis (Melastomataceae), A New Species from Guangxi, China. Nordic Journal of Botany 38 (e02525): 1-3. 2020 [2] doi: 10.1111/njb.02525

CHINA (Guangxi*)

Melastoma candidum D. Don f. albiflorum J. C. Ou 白花野牡丹 [Typification]

ZHANG Xin-jian, DAI Jin-hong, LIU Xiao-zhou, LI Zi-hua, LEE Shiou-yih, ZHOU Ren-chao, TAN Guang-wen, Lectotypification of the Name Melastoma candidum f. albiflorum and Its Taxonomic Status. PhytoKeys 146: 47-52. 2020 [50] doi: 10.3897/phytokeys.146.49929

CHINA (Taiwan*)

\section{Meliaceae 楝科}

Toona fargesii A. Chev. 红花香椿 [Correction]

A.: Toona ciliata M. Roem. var. pubescens auct. non. (Franch.) Hand.-Mazz.: Z. Wei \& Y. B. Zhang in Z. Wei \& Y. Q. He, Fl. Zhejiang 3: 452.1993

CHEN Feng, XIE Wen-yuan, ZHANG Shui-li, ZHANG Fen-yao, CHEN Zheng-hai, Additional Notes on the Seed Plants in Zhejiang (II). Journal of Hangzhou Normal University (Natural Science Edition) 19 (6): 96-102. 2020 [97] doi: 10.12191/j.issn.1674232X.2020.06.014

CHINA (Zhejiang)

\section{Meteoriaceae 蔓蘚科}


杜诚, 刘军, 叶文, 廖帅, 葛斌杰, 刘冰, 马金双 (2021) 中国植物新分类群、新名称 2020 年度报告. 生物多样性, 29, 10111020. http://www.biodiversity-science.net/CN/10.17520/biods.2021122

Neodicladiella capillicaulis (Renauld \& Cardot) G. W. Naren \& Y. Jia [New Combination]

B.: Aerobryum capillicaule Renauld \& Cardot

NAREN Gao Wa, JIA Yu, Taxonomic Study on Meteoriaceae I. Reappraisal of Some Species of Barbella and Pseudotrachypus. Journal of Bryology 42 (4): 333-342. 2020 [341] doi: 10.1080/03736687.2020.1839247

CHINA (Fujian, Guangxi, Jiangxi, Yunnan); JAPAN, MADAGASCAR*

Neodicladiella capillicaulis (Renauld \& Cardot) G. W. Naren \& Y. Jia [New Record]

NAREN Gao Wa, JIA Yu, Taxonomic Study on Meteoriaceae I. Reappraisal of Some Species of Barbella and Pseudotrachypus. Journal of Bryology 42 (4): 333-342. 2020 [341] doi: 10.1080/03736687.2020.1839247

CHINA (Fujian, Guangxi, Jiangxi, Yunnan); JAPAN, MADAGASCAR*

\section{Mniaceae 提灯蘚科}

Orthomnion elimbatum (Nog.) T. J. Kop. [New Record]

YI Yan-jun, XIAO Xiao-xuan, LI Yan, HE Si, Orthomnion elimbatum (Mniaceae, Bryophyta), a Malesian and Australian Species New to China and Vietnam Based on Molecular Data. Phytotaxa 451 (3): 206-214. 2020 [209] doi: 10.11646/phytotaxa.451.3.3

CHINA (Xizang, Yunnan); PAPUA NEW GUINEA

Pohlia tundrae A. J. Shaw 冻原丝瓜蘚[New Record]

WANG Xiao-rui, FAN Ying-jie, ZHAO Jian-cheng, SHAO Xiao-ming, Pohlia tundrae A. J. Shaw (Mielichhoferiaceae, Bryophyta), a New Recorded Species to China. Acta Botanica Boreali-Occidentalia Sinica 40 (6): 1070-1074. 2020 [1071] doi: 10.7606/j.issn.1000-4025.2020.06.1070

CHINA (Xizang); [ASIA], [EUROPE]

\section{Moraceae 桑科}

Ficus howii Merr. \& Chun [New Synonyms]

S.: Ficus pubigera (Miq.) Wall. ex Brandis var. reticulata S. S. Chang

ZHANG Zhen, LIAO Shuai, LI Hong-qing, ZHANG De-shun, Identifications and Typifications of Ficus pubigera var. maliformis and Related Names. Phytotaxa 464 (1): 102-108. 2020 [105] doi: 10.11646/phytotaxa.464.1.9

CHINA (Guangxi, Guizhou, Hainan, Xizang, Yunnan*); INDIA, LAOS, MYANMAR, THAILAND

Ficus jingningensis X. D. Mei, Z. H. Chen \& G. Y. Li 景宁榕 [New Species] IUCN: NE; Evidence: Morphology

CHEN Zheng-hai, MEI Xu-dong, CHEN Feng, XIE Wen-yuan, LI Gen-you, Ficus jingningensis (Moraceae), a New Species from Zhejiang. Journal of Zhejiang Forestry Science and Technology 40 (5): 52-54. 2020 [53] doi: 10.3969/j.issn.1001-3776.2020.05.009 CHINA (Zhejiang*)

Ficus pubigera (Miq.) Wall. ex Brandis var. maliformis (King) Corner [New Synonyms]

S.: Ficus yunnanensis S. S. Chang

ZHANG Zhen, LIAO Shuai, LI Hong-qing, ZHANG De-shun, Identifications and Typifications of Ficus pubigera var. maliformis and

Related Names. Phytotaxa 464 (1): 102-108. 2020 [102] doi: 10.11646/phytotaxa.464.1.9

CHINA (Yunnan); INDIA*, MYANMAR

Ficus pubigera (Miq.) Wall. ex Brandis var. maliformis (King) Corner [Typification]

ZHANG Zhen, LIAO Shuai, LI Hong-qing, ZHANG De-shun, Identifications and Typifications of Ficus pubigera var. maliformis and Related Names. Phytotaxa 464 (1): 102-108. 2020 [102] doi: 10.11646/phytotaxa.464.1.9

CHINA (Yunnan); INDIA*, MYANMAR

Maclura montana Z. P. Lei, G. Y. Li \& Z. H. Chen 山地柘 [New Species] IUCN: NE; Evidence: Morphology

LEI Zu-pei, CHEN Zheng-hai, XIE Wen-yuan, MEI Xu-dong, LIN Li-si, LI Gen-you, A New Species of Maclura Nutt. (Moraceae) from Mountains in Southern Zhejiang. Journal of Zhejiang Forestry Science and Technology 40 (6): 61-63. 2020 [62] doi: 10.3969/j.issn.1001-3776.2020.06.010

CHINA (Zhejiang*)

Maclura orientalis G. Y. Li, W. Y. Xie \& Z. H. Chen 东部藤柘 [New Species] IUCN: NE; Evidence: Morphology

CHEN Zheng-hai, LEI Zu-pei, XIE Wen-yuan, MEI Xu-dong, LIU Xi, LI Gen-you, A New Species of Maclura Nutt. (Moraceae) from China. Journal of Zhejiang Forestry Science and Technology 40 (5): 55-58. 2020 [56] doi: 10.3969/j.issn.1001-3776.2020.05.010 CHINA (Zhejiang*)

\section{Myrtaceae 桃金娘科}

Syzygium jambos (L.) Alston [Typification] 
杜诚, 刘军, 叶文, 廖帅, 葛斌杰, 刘冰, 马金双 (2021) 中国植物新分类群、新名称 2020 年度报告. 生物多样性, 29, 10111020. http://www.biodiversity-science.net/CN/10.17520/biods.2021122

SINGH Rajeev Kumar, ARIGELA Ravi kiran, Lectotypifications and New Synonyms in Indian Syzygium (Myrtaceae). Phytotaxa 429 (3): 200-216. 2020 [210] doi: 10.11646/phytotaxa.429.3.2

CHINA (Yunnan); INDIA, SRI LANKA*

Syzygium tetragonum Wall. ex Wight [Typification]

SINGH Rajeev Kumar, ARIGELA Ravi kiran, Lectotypifications and New Synonyms in Indian Syzygium (Myrtaceae). Phytotaxa 429 (3): 200-216. 2020 [213] doi: 10.11646/phytotaxa.429.3.2

CHINA (Guangxi, Hainan, Xizang, Yunnan); BHUTAN, INDIA*, MYANMAR, NEPAL, THAILAND

Syzygium verticillatum (C. Chen) G. Y. Li \& Z. H. Chen 轮叶赤楠 [New Combination]

B.: Syzygium buxifolium Hook. \& Arn. var. verticillatum C. Chen

CHEN Zheng-hai, ZHOU Qing, CHEN Gao-kun, XIE Wen-yuan, LI Gen-you, Notes on Seed Plant in Zhejiang Province (VI). Journal of Zhejiang Forestry Science and Technology 40 (4): 73-77. 2020 [76] doi: 10.3969/j.issn.1001-3776.2020.04.013

CHINA (Anhui, Fujian, Guangdong, Guangxi*, Guizhou, Hunan, Jiangxi, Zhejiang)

Syzygium tetragonum (L.) DC. [Typification]

SINGH Rajeev Kumar, ARIGELA Ravi kiran, Lectotypifications and New Synonyms in Indian Syzygium (Myrtaceae). Phytotaxa 429 (3): 200-216. 2020 [213] doi: 10.11646/phytotaxa.429.3.2

CHINA (Guangdong, Guangxi); CAMBODIA, INDIA, INDONESIA, LAOS, MALAYSIA, MYANMAR, SRI LANKA*, THAILAND, VIETNAM

\section{Oleaceae 木樨科}

Chengiodendron C. B. Shang, X. R. Wang, Yi F. Duan \& Yong F. Li [New Genus]

LI Yong-fu, ZHANG Min, WANG Xian-rong, SYLVESTER Steven Paul, XIANG Qi-bai, LI Xuan, LI Meng, ZHU Hong, ZHANG Cheng, CHEN Lin, YI Xian-gui, MAO Ling-feng, DUAN Yi-fan, Revisiting the phylogeny and Taxonomy of Osmanthus (Oleaceae) including Description of the New Genus Chengiodendron. Phytotaxa 436 (3): 283-292. 2020 [289] doi: 10.11646/phytotaxa.436.3.6 CHINA

Chengiodendron marginatum (Champ. ex Benth.) C. B. Shang, X. R. Wang, Yi F. Duan \& Yong F. Li [New Combination]

B.: Olea marginata Champ. ex Benth.

LI Yong-fu, ZHANG Min, WANG Xian-rong, SYLVESTER Steven Paul, XIANG Qi-bai, LI Xuan, LI Meng, ZHU Hong, ZHANG Cheng, CHEN Lin, YI Xian-gui, MAO Ling-feng, DUAN Yi-fan, Revisiting the phylogeny and Taxonomy of Osmanthus (Oleaceae) including Description of the New Genus Chengiodendron. Phytotaxa 436 (3): 283-292. 2020 [289] doi: 10.11646/phytotaxa.436.3.6 CHINA (Hong Kong*)

Chengiodendron matsumuranum (Hayata) C. B. Shang, X. R. Wang, Yi F. Duan \& Yong F. Li [New Combination]

B.: Osmanthus matsumuranus Hayata

LI Yong-fu, ZHANG Min, WANG Xian-rong, SYLVESTER Steven Paul, XIANG Qi-bai, LI Xuan, LI Meng, ZHU Hong, ZHANG Cheng, CHEN Lin, YI Xian-gui, MAO Ling-feng, DUAN Yi-fan, Revisiting the phylogeny and Taxonomy of Osmanthus (Oleaceae) including Description of the New Genus Chengiodendron. Phytotaxa 436 (3): 283-292. 2020 [290] doi: 10.11646/phytotaxa.436.3.6 CHINA (Anhui, Guangdong, Guangxi, Guizhou, Jiangxi, Taiwan, Yunnan, Zhejiang); CAMBODIA, INDIA, VIET NAM*

Chengiodendron minor (P. S. Green) C. B. Shang, X. R. Wang, Yi F. Duan \& Yong F. Li [New Combination]

B.: Osmanthus minor P. S. Green

LI Yong-fu, ZHANG Min, WANG Xian-rong, SYLVESTER Steven Paul, XIANG Qi-bai, LI Xuan, LI Meng, ZHU Hong, ZHANG Cheng, CHEN Lin, YI Xian-gui, MAO Ling-feng, DUAN Yi-fan, Revisiting the phylogeny and Taxonomy of Osmanthus (Oleaceae) including Description of the New Genus Chengiodendron. Phytotaxa 436 (3): 283-292. 2020 [290] doi: 10.11646/phytotaxa.436.3.6 CHINA (Guangdong*)

Jasminum chiae Kai Zhang \& D. X. Zhang [New Species] IUCN: CR; Evidence: Morphology

ZHANG Kai, WU Ming-song, QIAN Xin, ZHANG Dian-xiang, Jasminum chiae sp. nov. (Oleaceae), from a Tropical Limestone Area in Yunnan, China. Nordic Journal of Botany 38 (e02857): 1-6. 2020 [3] doi: 10.1111/njb.02857

CHINA (Yunnan*)

Jasminum parceflorum Kai Zhang \& D. X. Zhang [New Species] IUCN: VU: D1; Evidence: Morphology

ZHANG Kai, WU Ming-song, PAN Bo, ZHOU Lian-xuan, ZHANG Dian-xiang, Jasminum parceflorum (Oleaceae), a New Species from Southern Yunnan, China. PhytoKeys 146: 109-115. 2020 [110] doi: 10.3897/phytokeys.146.49625 CHINA (Yunnan*)

Ligustrum purpurascens Yen C. Yang 紫枝女贞 [Correction]

A.: Ligustrum robustum auct., non (Roxb.) Blume: M. C. Chang \& L. Q. Qiu in Fl. Reipubl. Popularis Sin. 61: 155.1992.

CHEN Lin, ZHEN Shuang-long, XIE Wen-yuan, LIN Feng, CHEN Zheng-hai, Additional Notes on the Seed Plant Flora in Zhejiang (V). Journal of Wenzhou University (Natural Science Edition) 41 (4): 33-38. 2020 [33] doi: 10.3875/j.issn.1674-3563.2020.04.006 
杜诚, 刘军, 叶文, 廖帅, 葛斌杰, 刘冰, 马金双 (2021) 中国植物新分类群、新名称 2020 年度报告. 生物多样性, 29, 10111020. http://www.biodiversity-science.net/CN/10.17520/biods.2021122

CHINA (Anhui, Fujian, Gangdong, Guangxi, Guizhou, Hubei, Hunan, Jiangxi, Sichuan*, Yunnan)

\section{Onagraceae 柳叶菜科}

Chamaenerion speciosum (Decaisne) Hoch \& K. Gandhi [New Combination]

B.: Epilobium speciosum Decaisne

HOCH Peter C., GANDHI Kanchi, Nomenclatural Changes in Onagraceae. PhytoKeys 145: 57-62. 2020 [60] doi: 10.3897/phytokeys.145.51139

CHINA (Xizang); INDIA*

\section{Ophioglossaceae 瓶尔小草科}

Sahashia Li Bing Zhang \& Liang Zhang [New Genus]

ZHANG Liang, FAN Xue - ping, PETCHSRI Sahanat, ZHOU Lin, POLLAWATN Rossarin, ZHANG Xin, ZHOU Xin - mao, LU Ngan Thi, KNAPP Ralf, CHANTANAORRAPINT Sahut, LIMPANASITTICHAI Ponpipat, SUN Hang, GAO Xin - fen, ZHANG $\mathrm{Li}$ - bing, Evolutionary Relationships of the Ancient Fern Lineage the Adder's Tongues (Ophioglossaceae) with Description of Sahashia gen. nov. Cladistics 36 (4): 380-393. 2020 [387] doi: 10.1111/cla.12408

CHINA

Sahashia stricta (Underw.) Li Bing Zhang \& Liang Zhang [New Combination]

B.: Botrychium strictum Underw.

ZHANG Liang, FAN Xue - ping, PETCHSRI Sahanat, ZHOU Lin, POLLAWATN Rossarin, ZHANG Xin, ZHOU Xin - mao, LU Ngan Thi, KNAPP Ralf, CHANTANAORRAPINT Sahut, LIMPANASITTICHAI Ponpipat, SUN Hang, GAO Xin - fen, ZHANG $\mathrm{Li}$ - bing, Evolutionary Relationships of the Ancient Fern Lineage the Adder's Tongues (Ophioglossaceae) with Description of Sahashia gen. nov. Cladistics 36 (4): 380-393. 2020 [388] doi: 10.1111/cla.12408

CHINA (Chongqing, Gansu, Heilongjiang, Henan, Hubei, Jilin, Liaoning, Nei Mongol, Shaanxi, Sichuan); KOREA, JAPAN*, RUSSIA

\section{Orchidaceae 兰科}

Arundina graminifolia (D. Don) Hochr. var. jinpingensis W. H. Rao \& Y. Y. Pan 金平竹叶兰 [New Variety]

RAO Wen-hui, CHEN Jian-bing, KONG De-min, HUANG Jie, LI Li-qiang, PAN Yun-yun, "Arundina graminfolia var. jinpingensis, a New Variety of Arundina from Yunnan, China". Forest Science and Technology (8): 101-102. 2020 [101] doi: 10.13456/j.cnki.lykt.2019.08.08.0001

CHINA (Yunnan*)

Blepharoglossum (Schltr.) L. Li [New Genus]

LI Lin, CHUNG Shih-wen, LI Bo, ZENG Song-jun, YAN Hai-fei, LI Shi-jin, New insight into the Molecular Phylogeny of the Genus Liparis s. l. (Orchidaceae: Malaxideae) with a New Generic Segregate: Blepharoglossum. Plant Systematics and Evolution 306 (54): 1-10. 2020 [6] doi: 10.1007/s00606-020-01679-3

CHINA

Blepharoglossum condylobulbon (Rchb. f.) L. Li [New Combination]

B.: Liparis condylobulbon Rchb. f.

LI Lin, CHUNG Shih-wen, LI Bo, ZENG Song-jun, YAN Hai-fei, LI Shi-jin, New insight into the Molecular Phylogeny of the Genus Liparis s. l. (Orchidaceae: Malaxideae) with a New Generic Segregate: Blepharoglossum. Plant Systematics and Evolution 306 (54): 1-10. 2020 [7] doi: 10.1007/s00606-020-01679-3

CHINA (Taiwan); INDIA, INDONESIA*, PHILIPPINES

Blepharoglossum fissilabrum (T. Tang \& F. T. Wang) L. Li [New Combination]

B.: Liparis fissilabris T. Tang \& F. T. Wang

LI Lin, CHUNG Shih-wen, LI Bo, ZENG Song-jun, YAN Hai-fei, LI Shi-jin, New insight into the Molecular Phylogeny of the Genus Liparis s. l. (Orchidaceae: Malaxideae) with a New Generic Segregate: Blepharoglossum. Plant Systematics and Evolution 306 (54): 1-10. 2020 [8] doi: 10.1007/s00606-020-01679-3

CHINA (Hainan)

Blepharoglossum grossum (Rchb. f.) L. Li [New Combination]

B.: Liparis grossa Rchb. f.

LI Lin, CHUNG Shih-wen, LI Bo, ZENG Song-jun, YAN Hai-fei, LI Shi-jin, New insight into the Molecular Phylogeny of the Genus Liparis s. l. (Orchidaceae: Malaxideae) with a New Generic Segregate: Blepharoglossum. Plant Systematics and Evolution 306 (54): 1-10. 2020 [8] doi: 10.1007/s00606-020-01679-3

CHINA (Taiwan); MYANMAR* 
杜诚, 刘军, 叶文, 廖帅, 葛斌杰, 刘冰, 马金双 (2021) 中国植物新分类群、新名称 2020 年度报告. 生物多样性, 29, 10111020. http://www.biodiversity-science.net/CN/10.17520/biods.2021122

Blepharoglossum latifolium (Lindl.) L. Li [New Combination]

B.: Liparis latifolia Lindl.

LI Lin, CHUNG Shih-wen, LI Bo, ZENG Song-jun, YAN Hai-fei, LI Shi-jin, New insight into the Molecular Phylogeny of the Genus Liparis s. l. (Orchidaceae: Malaxideae) with a New Generic Segregate: Blepharoglossum. Plant Systematics and Evolution 306 (54): 1-10. 2020 [8] doi: 10.1007/s00606-020-01679-3

CHINA (Hainan); INDONESIA*, MALAYSIA

Brachycorythis menglianensis Y. Y. Qian 孟连苞叶兰 [Rediscovery]

WANG Yu-qian, LIU Qiang, WU Xun-feng, LI Jin-long, WANG Bo-yi, PAN Bo, Rediscovery of Brachycorythis menglianensis (Orchidaceae) with Additional Descriptive Notes. Guihaia 40 (11): 1623-1627. 2020 [1624] doi: 10.11931/guihaia.gxzw201906049 CHINA (Yunnan*)

Bulbophyllum gedangense Y. Luo, J. P. Deng \& Jian W. Li [New Species] IUCN: DD; Evidence: Morphology

LUO Yan, DENG Jian-ping, PENG Yu-lan, YAN Li-chun, LI Jian-wu, Bulbophyllum gedangense (Orchidaceae, Epidendroideae, Malaxideae), a New Species from Tibet, China. Phytotaxa 453 (2): 145-150. 2020 [146] doi: 10.11646/phytotaxa.453.2.6 CHINA (Xizang*)

Bulbophyllum lingii M. Z. Huang, G. S. Yang \& J. M. Yin 凌氏石豆兰 [New Species] IUCN: DD; Evidence: Morphology HUANG Ming-zhong, YANG Guang-sui, LAN Si-ren, YIN Jun-mei, Bulbophyllum lingii, a New Species (Malaxideae, Epidedroideae, Orchidaceae) from Hainan, China. Phytotaxa 452 (2): 185-188. 2020 [185] doi: 10.11646/phytotaxa.452.2.8

CHINA (Hainan*)

Bulbophyllum linzhiense Liang Ma \& S. P. Chen 林芝石豆兰 [New Species] IUCN: CR; Evidence: ITS, matK, atpI-atpH, trnL-F; Morphology

MA Liang, ZHANG Sai, ZHOU Zhuang, LAN Si-ren, CHEN Shi-pin, Bulbophyllum linzhiense (Orchidaceae, Malaxideae), a New Species from China. Phytotaxa 429 (4): 281-288. 2020 [287] doi: 10.11646/phytotaxa.429.4.4

CHINA (Xizang*)

Bulbophyllum omerandrum Hayata var. macropetalum Liang Ma, Xin Y. Chen \& S. P. Chen 大瓣卷瓣兰 [New Variety]

MA Liang, CHEN Xin-yan, TU Xiong-de, CHEN Shi-pin, LAN Si-ren, Bulbophyllum omerandrum var. macropetalum, a New Variety of Orchidaceae from Fujian, China. Journal of Fujian Agriculture and Forestry University (Natural Science Edition) 49 (5): $597-$ 599. 2020 [597] doi: 10.13323/j.cnki.j.fafu(nat.sci.)2020.05.004

CHINA (Fujian*)

Calanthe sieboldopsis Bo Y. Yang \& Bo Li 异大黄花虾脊兰 [New Species] IUCN: CR: D; Evidence: Morphology

YANG Bo-yun, LUO Huo-lin, HUANG Wei-chang, XIONG Dong-jin, TAN Shao-lin, LI Bo, Calanthe sieboldopsis (Orchidaceae, Epidendroideae, Collabieae), a New Species from Luoxiao Mountains, Eastern China. PhytoKeys 145: 37-45. 2020 [39] doi: 10.3897/phytokeys.145.49386

CHINA (Jiangxi*)

Calanthe tsiana Y. Q. Chen, J. W. Zhai \& S. R. Lan 吉氏虾脊兰 [New Species] IUCN: NE; Evidence: rITS, matK, trnL-trnF, rbcL; Morphology

CHEN Yan-qiong, ZHU Ya-ting, ZHONG Hui, LI Ze-xin, LIU Zhong-jian, ZHAI Jun-wen, LAN Si-ren, Calanthe tsiana, a New Orchid Species from China (Epidendroideae: Collabieae): Evidence from Morphological and Molecular Analyses. Phytotaxa 428 (1): $67-$ 72. 2020 [70] doi: 10.11646/phytotaxa.428.1.7

CHINA (Yunnan*)

Cheirostylis barbata Q. Liu \& X. F. Wu 流苏叉柱兰 [New Species] IUCN: DD; Evidence: Morphology

WU Xun-feng, LIU Qiang, LI Jin-long, TANG Zong-ying, XING Xiao-ke, Cheirostylis barbata, a New Species Orchidaceae (Goodyerinae) from Yunnan, China. Phytotaxa 436 (3): 293-297. 2020 [290] doi: 10.11646/phytotaxa.436.3.7

CHINA (Yunnan*)

Cheirostylis liukiuensis Masam. var. nantouensis (T. P. Lin) T. P. Lin 南投指柱兰 [New Combination]

B.: Cheirostylis nantouensis T. P. Lin

LIN Tsan-piao, New Additions of Oreorchis, Cheirostylis, and Cymbidium (Orchidaceae) from Taiwan. Taiwania 65 (4): $463-472$. 2020 [471] doi: 10.6165/tai.2020.65.463

CHINA (Taiwan*)

Cheirostylis tortilacinia C. S. Leou var. rubrifolia (T. P. Lin \& W. M. Lin) T. P. Lin 红衣指柱兰 [New Combination]

B.: Cheirostylis rubrifolia T. P. Lin \& W. M. Lin

LIN Tsan-piao, New Additions of Oreorchis, Cheirostylis, and Cymbidium (Orchidaceae) from Taiwan. Taiwania 65 (4): $463-472$. 2020 [469] doi: 10.6165/tai.2020.65.463

CHINA (Taiwan*)

Cheirostylis tortilacinia C. S. Leou var. wutaiensis T. P. Lin 拟红衣指柱兰 [New Variety] 
杜诚, 刘军, 叶文, 廖帅, 葛斌杰, 刘冰, 马金双 (2021) 中国植物新分类群、新名称 2020 年度报告. 生物多样性, 29, 10111020. http://www.biodiversity-science.net/CN/10.17520/biods.2021122

LIN Tsan-piao, New Additions of Oreorchis, Cheirostylis, and Cymbidium (Orchidaceae) from Taiwan. Taiwania 65 (4): $463-472$. 2020 [469] doi: 10.6165/tai.2020.65.463

CHINA (Taiwan*)

Cionisaccus procera (Ker Gawl.) M. C. Pace [New Combination]

B.: Neottia procera Ker Gawl.

PACE Matthew C., A Recircumscription of Goodyera (Orchidaceae), including the Description of Paorchis gen. nov., and Resurrection of Cionisaccus, Eucosia, and Salacistis. Brittonia 72 (3): 257-267. 2020 [263] doi: 10.1007/s12228-020-09623-y

CHINA (Anhui, Fujian, Guangdong, Guangxi, Guizhou, Hainan, Sichuan, Taiwan, Xizang, Yunnan, Zhejiang); BANGLADESH, BHUTAN, CAMBODIA, INDIA, INDONESIA, JAPAN, LAOS, MYANMAR, NEPAL*, PHILIPPINES, SRI LANKA, THAILAND, VIETNAM

Cleisostoma hainanense M. Z. Huang, D. K. Liu \& G. S. Yang 海南隔距兰 [New Species] IUCN: Endangered (No evidence); Evidence: nrITS, atpI-atpH, matK, psbA-trnH, trnL-F; Morphology

HUANG Ming-zhong, LIU Ding-kun, YIN Jun-mei, YANG Guang-sui, Cleisostoma hainanense, a New Species (Orchidaceae: Epidendroideae) from Hainan, China: Evidence from Morphological and DNA Analyses. Phytotaxa 428 (3): 263-270. 2020 [270] doi: 10.11646/phytotaxa.428.3.7

CHINA (Hainan*)

Crepidium josephianum (Rchb.f.) Marg. 大花沼兰 [New Record]

LIU Wen-jian, WANG Li-yan, WU Lei, JIN Xiao-hua, Crepidium josephianum, a New Record Of Orchidaceae from China. Plant Science Journal 38 (3): 316-319. 2020 [317] doi: 10.11913/PSJ.2095-0837.2020.30316

CHINA (Yunnan); INDIA*

Cymbidium $\times$ malipoense L. J. Chen, Hong B. Zhou \& Z. J. Liu 麻栗坡长叶兰 [New Nothospecies]

H.: Cymbidium erythraeum Lindl. $\times$ Cymbidium eburneum Lindl. var. longzhouense Z. J. Liu \& S. C. Chen

CHEN Li-jun, ZHOU Hong-bo, LIU Zhong-jian, ZHENG Fang, WANG Yu, RAO Wen-hui, Cymbidium $\times$ malipoense a New Hybrid of Orchidaceae from Yunnan China. Plant Science Journal 38 (2): 181-184. 2020 [182] doi: 10.11913/PSJ.2095-0837.2020.20181 CHINA (Yunnan*)

Cymbidium biflorens D. Y. Zhang, S. R. Lan \& Z. J. Liu 两季兰 [New Species] IUCN: Endangered (No evidence); Evidence: nrITS, matK, rbcL; Morphology

ZHANG Di-yang, TU Xiong-de, LIU Bin, LIAO Xing-yu, LAN Si-ren, LIU Zhong-jian, Cymbidium biflorens (Orchidaceae; Epidendroideae), a New Species from China: Evidence from Morphological and Molecular Data. Phytotaxa 428 (3): 271-278. 2020 [274] doi: 10.11646/phytotaxa.428.3.8

CHINA (Yunnan*)

Cymbidium brevifolium Zhuang Zhou, S. R. Lan \& Z. J. Liu 小惠兰 [New Species] IUCN: NE; Evidence: nrITS, matK, rbcL; Morphology

ZHOU Zhuang, ZHANG Di-yang, CHEN Gui-zhen, YU Xia, LI Cheng-ru, LAN Si-ren, LIU Zhong-jian, Cymbidium brevifolium (Orchidaceae; Epidendroideae), a New Species from China: Evidence from Morphological and Molecular Data. Phytotaxa 464 (3): 236-242. 2020 [240] doi: 10.11646/phytotaxa.464.3.5

CHINA (Hubei*)

Cymbidium codonanthum Y. T. Jiang, Liang Ma \& S. P. Chen 钟花兰 [New Species] IUCN: Endangered (No evidence); Evidence: nrITS, matK, rbcL; Morphology

JIANG Yu-ting, MA Liang, LIN Ru-qiang, XIONG Yuan-fang, JIANG Shu-zhen, LIU Bin, LIU Zhong-Jian, CHEN Shi-pin, Cymbidium codonanthum (Orchidaceae; Epidendroideae; Cymbidiinae), a New Species from China: Evidence from Morphological and Molecular Analyses. Phytotaxa 453 (3): 275-283. 2020 [281] doi: 10.11646/phytotaxa.453.3.9

CHINA (Yunnan*)

Cymbidium dayanum Rchb. f. f. albiflorum (S. S. Ying) O. Gruss \& N. H. Tuan [New Combination]

B.: Cymbidium dayanum Rchb. f. var. albiflorum S. S. Ying

TUAN Nguyen Hoang, GRUSS Olaf, HUYEN Le Dang, CANH Chu Xuan, Cymbidium dayanum f. flavoviride, eine Gelbgrüne Farbform aus Vietnam. Die Orchidee (Herausgeber) 6 (24): 201-208. 2020 [205]

CHINA (Taiwan)

Cymbilabia D. K. Liu \& Ming H. Li [New Genus]

LIU Ding-kun, TU Xiong-de, ZHAO Zhuang, ZENG Meng-yao, ZHANG Sai, MA Liang, ZHANG Guo-qiang, WANG Miao-miao, LIU Zhong-jian, LAN Si-ren, LI Ming-he, CHEN Shi-pin, Plastid Phylogenomic Data Yield New and Robust Insights into the Phylogeny of Cleisostoma-Gastrochilus Clades (Orchidaceae, Aeridinae). Molecular Phylogenetics and Evolution 145 (106729): 19. 2020 [7] doi: 10.1016/j.ympev.2019.106729

CHINA 
杜诚, 刘军, 叶文, 廖帅, 葛斌杰, 刘冰, 马金双 (2021) 中国植物新分类群、新名称 2020 年度报告. 生物多样性, 29, 10111020. http://www.biodiversity-science.net/CN/10.17520/biods.2021122

Cymbilabia undulata (Lindl.) D. K. Liu \& Ming H. Li [New Combination]

B.: Vanda undulata Lindl.

LIU Ding-kun, TU Xiong-de, ZHAO Zhuang, ZENG Meng-yao, ZHANG Sai, MA Liang, ZHANG Guo-qiang, WANG Miao-miao, LIU Zhong-jian, LAN Si-ren, LI Ming-he, CHEN Shi-pin, Plastid Phylogenomic Data Yield New and Robust Insights into the Phylogeny of Cleisostoma-Gastrochilus Clades (Orchidaceae, Aeridinae). Molecular Phylogenetics and Evolution 145 (106729): 19. 2020 [7] doi: 10.1016/j.ympev.2019.106729

CHINA (Xizang, Yunnan)

Cypripedium segawae Masam. 宝岛构兰 [New Record]

ZHANG Di-yang, LIU Zhong-jian, YIN Wei-lun, LAN Si-ren, Cypripedium segawae, a New Record of Cypripedium (Orchidaceae) from Mainland China. Journal of Fujian Agriculture and Forestry University (Natural Science Edition) 49 (6): 779-781. 2020 [780] doi: 10.13323/j.cnki.j.fafu(nat.sci.)2020.06.010

CHINA (Gansu, Taiwan)

Dendrobium jinghuanum B. Q. Zheng \& Yan Wang 景华石斛 [New Species] IUCN: EN: D; Evidence: nrITS, matK, trnL, rbcL; Morphology

ZHENG Bao-qiang, ZOU Long-hai, WAN Xiao, WANG Yan, Dendrobium jinghuanum, a New Orchid Species from Yunnan, China: Evidence from both Morphology and DNA. Phytotaxa 428 (1): 30-42. 2020 [40] doi: 10.11646/phytotaxa.428.1.3

CHINA (Yunnan*)

Dendrobium naungmungense Q. Liu \& X. H. Jin 瑙蒙石斛 [New Record]

MA Liang, DONG Jian-wen, CHEN Shi-pin, ZHUANG Li-bin, Dendrobium naungmungense Q. Liu \& X. H. Jin, a New Record of Orchidaceae from China. Journal of Tropical and Subtropical Botany 28 (2): 201-202. 2020 [201] doi: 10.11926/jtsb.4109

CHINA (Yunnan); MYANMAR*

Dendrobium yongjiaense Zhuang Zhou \& S. R. Lan 永嘉石斛 [New Species] IUCN: CR; Evidence: nrITS, matK, trnL-F; Morphology

ZHOU Zhuang, ZHANG Sai, YANG Yan-ping, MA Liang, LAN Si-ren, Morphological and Molecular Evidence for a New Species from China: Dendrobium yongjiaense (Orchidaceae: Malaxideae). Phytotaxa 441 (2): 203-210. 2020 [209] doi: 10.11646/phytotaxa.441.2.7

CHINA (Zhejiang*)

Epipogium taiwanense T. C. Hsu 台湾虎舌兰 [New Record]

WU Xun-feng, LIU Qiang, TANG Ying, CHI Kuan-bo, WANG Yu-qian, PANG Xin, HUANG Yao-hua, PENG Pei-hao, Epipogium taiwanense, a Detail Morphological Description and Additional New Record to Mainland China. Phytotaxa 453 (1): $43-48.2020$ [44] doi: 10.11646/phytotaxa.453.1.4

CHINA (Sichuan, Taiwan*, Yunnan)

Eucosia seikomontana (Yamam.) M. C. Pace [New Combination]

B.: Goodyera seikomontana Yamam.

PACE Matthew C., A Recircumscription of Goodyera (Orchidaceae), including the Description of Paorchis gen. nov., and Resurrection of Cionisaccus, Eucosia, and Salacistis. Brittonia 72 (3): 257-267. 2020 [263] doi: 10.1007/s12228-020-09623-y

CHINA (Hong Kong, Taiwan*)

Eucosia viridiflora (Blume) M. C. Pace [New Combination]

B.: Neottia viridiflora Blume

PACE Matthew C., A Recircumscription of Goodyera (Orchidaceae), including the Description of Paorchis gen. nov., and Resurrection of Cionisaccus, Eucosia, and Salacistis. Brittonia 72 (3): 257-267. 2020 [264] doi: 10.1007/s12228-020-09623-y

CHINA (Fujian, Guangdong, Hainan, Jiangxi, Taiwan, Yunnan); BHUTAN, INDIA, INDONESIA*, S JAPAN, MALAYSIA, NEPAL, NEW GUINEA, PHILIPPINES, THAILAND, VIETNAM; AUSTRALIA

Gastrodia flexistyla T. C. Hsu \& C. M. Kuo 折柱天麻 [New Record]

QIN Ying, CHEN Hai-ling, HUANG Yu-song, LIU Yan, New Record of Gastrodia (Orchidaceae) from Mainland, China. Acta Botanica Boreali-Occidentalia Sinica 40 (7): 1255-1258. 2020 [1255] doi: 10.7606/j.issn.1000-4025.2020.07.1255

CHINA (Guangxi, Taiwan*)

Gastrodia shimizuana Tuyama 叉脊天麻 [New Record]

QIN Ying, CHEN Hai-ling, HUANG Yu-song, LIU Yan, New Record of Gastrodia (Orchidaceae) from Mainland, China. Acta Botanica Boreali-Occidentalia Sinica 40 (7): 1255-1258. 2020 [1256] doi: 10.7606/j.issn.1000-4025.2020.07.1255

CHINA (Guangxi, Taiwan*); JAPAN

Gastrodia uraiensis T. C. Hsu \& C. M. Kuo 乌来天麻 [New Record]

QIN Ying, LI Fu-wen, QIU Shao-jun, HUANG, Yu-zhao, LIU Yan, Gastrodia uraiensis, a Newly Recorded Species of Gastrodia from Mainland, China. Guihaia 40 (8): 1123-1126. 2020 [1124] doi: 10.11931/guihaia.gxzw201902016 
杜诚, 刘军, 叶文, 廖帅, 葛斌杰, 刘冰, 马金双 (2021) 中国植物新分类群、新名称 2020 年度报告. 生物多样性, 29, 10111020. http://www.biodiversity-science.net/CN/10.17520/biods.2021122

CHINA (Guangxi, Taiwan*)

Goodyera brachystegia Hand.-Mazz. [New Synomyms]

S.: Goodyera rosulacea Y. N. Lee

ZHOU Shan, ZHOU Xiao-xu, JIN Yue, SO Ji-Hyeon, LEE Nam-Sook, SCHUITEMAN André, KUMAR Pankaj, TIAN Huai-zhen, On the Identity of Goodyera rosulacea (Orchidaceae: Orchidoideae: Cranichideae: Goodyerinae). Kew Bulletin 75 (3): 1-6. 2020 [3] doi: 10.1007/S12225-019-9855-9

CHINA (Anhui, Gansu, Guizhou, Henan, Hubei, Shanxi, Shaanxi, Sichuan, Taiwan, Yunnan*, Xizang); KOREA

Goodyera nanshanensis Xi L. Wang \& X. H. Jin 南山斑叶兰 [New Species] IUCN: DD; Evidence: Morphology

WANG Xi-long, CHEN Juan, JIN Xiao-hua, Goodyera nanshanensis (Orchidaceae, Orchidoideae, Cranichideae, Goodyerinae), a New Species from Hunan, China. Phytotaxa 460 (4): 296-300. 2020 [296] doi: 10.11646/phytotaxa.460.4.7

CHINA (Hunan*)

Habenaria dentata Schltr. var. malipoense W. H. Rao \& Jie Huang tris 麻栗坡玉凤花 [New Variety]

RAO Wen-hui, CHEN Jian-bing, KONG De-min, HUANG Jie, PAN Yun-yun, LI Li-qiang, "Habenaria dentata var. malipoense, a New Variety of Habenaria from Yunnan, China". Forest Science and Technology (10): 88-88. 2020 [88] doi: 10.13456/j.cnki.lykt.2019.09.19.0006

CHINA (Yunnan*)

Liparis mae X. D. Tu, M. Z. Huang \& Ming H. Li 麻氏羊耳蒜 [New Species] IUCN: NE; Evidence: nrITS, matK; Morphology

TU Xiong-de, HUANG Ming-zhong, LIU Ding-kun, MA Liang, LI Ming-he, Liparis mai (Orchidaceae; Malaxideae), a New Species from China: Evidence from Morphological and Molecular Analyses. Phytotaxa 435 (3): 235-242. 2020 [236] doi: 10.11646/phytotaxa.435.3.3

CHINA (Guangxi*)

Luisia yunnanensis Jie Huang tris \& D. H. Peng 云南钗子股 [New Species] IUCN: NE; Evidence: nrITS, rbcL, matK; Morphology HUANG Jie, YU Yu-yun, CHEN Gui-zhen, LIAO Xing-yu, LIU Zhong-jian, PENG Dong-hui, Luisia yunnanensis (Orchidaceae; Epidendroideae), a New Species from China: Evidence from Morphology and DNA Analyses. Phytotaxa 475 (1): $52-58.2020$ [54] doi: 10.11646/phytotaxa.475.1.5

CHINA (Yunnan*)

Oberonia delacourii Gagnep. 凭祥盛尾兰 [New Record]

LI Yu-ling, LIN Ying, ZHENG Han-yue, WANG Bo, LIU Dong-ming, XING Fu-wu, Two Newly Recorded Species of Oberonia Lindl. (Orchidaceae) from China. Acta Botanica Boreali-Occidentalia Sinica 40 (9): 1613-1616. 2020 [1614] doi: 10.7606/j.issn.10004025.2020.09.1613

CHINA (Guangxi); LAOS*

Oberonia formosana Hayata f. viridiflora T. P. Lin 绿花台湾莪白兰 [New Form]

LIN Tsan-piao, New Additions of Oreorchis, Cheirostylis, and Cymbidium (Orchidaceae) from Taiwan. Taiwania 65 (4): $463-472$. 2020 [471] doi: 10.6165/tai.2020.65.463

CHINA (Taiwan*)

Oberonia integrilabris Yu L. Li \& F. W. Xing [New Species] IUCN: EN: B2ab(iii); Evidence: Morphology

LI Yu-ling, TONG Yi, YE De-ping, YAO Gang, XING Fu-wu, Oberonia integrilabris (Orchidaceae), a New Species from China. Phytotaxa 432 (1): 104-110. 2020 [105] doi: 10.11646/phytotaxa.432.1.9

CHINA (Yunnan*)

Oberonia orbicularis Hook. f. 心唇赹尾兰 [New Record]

LI Yu-ling, LIN Ying, ZHENG Han-yue, WANG Bo, LIU Dong-ming, XING Fu-wu, Two Newly Recorded Species of Oberonia Lindl. (Orchidaceae) from China. Acta Botanica Boreali-Occidentalia Sinica 40 (9): 1613-1616. 2020 [1614] doi: 10.7606/j.issn.10004025.2020.09.1613

CHINA (Yunnan); INDIA*

Oreorchis wumanae T. P. Lin 吴尾山兰 [New Species] IUCN: NE; Evidence: Morphology

LIN Tsan-piao, New Additions of Oreorchis, Cheirostylis, and Cymbidium (Orchidaceae) from Taiwan. Taiwania 65 (4): $463-472$. 2020 [463] doi: $10.6165 /$ tai.2020.65.463

CHINA (Taiwan*)

Paphiopedilum delenatii Guillaumin var. funingensis Y. Y. Pan \& W. H. Rao 富宁舀兰 [New Variety]

PAN Yun-yun, CHEN Jian-bing, KONG De-min, RAO Wen-hui, "Paphiopedilum delenatii var. funingensis, a New Variety of Paphiopedilum from Yunnan, China". Forest Science and Technology (8): 100-101. 2020 [100] doi: 10.13456/j.cnki.lykt.2019.08.14.0001

CHINA (Yunnan*) 
杜诚, 刘军, 叶文, 廖帅, 葛斌杰, 刘冰, 马金双 (2021) 中国植物新分类群、新名称 2020 年度报告. 生物多样性, 29, 10111020. http://www.biodiversity-science.net/CN/10.17520/biods.2021122

Paphiopedilum gratrixianum (Mast.) Rolfe var. simaoense W. H. Rao \& Jie Huang tris 思茅淣兰 [New Variety]

RAO Wen-hui, CHEN Jian-bing, KONG De-min, HUANG Jie, LI Li-qiang, Paphiopedilum gratrixianum var. simaoense, a New Variety of Paphiopedilum in China. Protection Forest Science and Technology (1): 75-76. 2020 [75] doi: 10.13601/j.issn.10055215.2020.01.029

CHINA (Yunnan*)

Platanthera jiuwanshanensis Ying Qin \& Yan Liu 九万山舌唇兰 [New Species] IUCN: DD; Evidence: Morphology

QIN Ying, HUANG Yu-song, MENG Tao, LIU Yan, Platanthera jiuwanshanensis (Orchidaceae), a New Species from Guangxi, China. Phytotaxa 436 (1): 72-78. 2020 [72] doi: 10.11646/phytotaxa.436.1.7

CHINA (Guangxi*)

Salacistis fumata (Thwaites) M. C. Pace [New Combination]

B.: Goodyera fumata Thwaites

PACE Matthew C., A Recircumscription of Goodyera (Orchidaceae), including the Description of Paorchis gen. nov., and Resurrection of Cionisaccus, Eucosia, and Salacistis. Brittonia 72 (3): 257-267. 2020 [265] doi: 10.1007/s12228-020-09623-y

CHINA (Hainan, Taiwan, Xizang, Yunnan); INDIA, INDONESIA, JAPAN, MALAYSIA, MYANMAR, PHILIPPINES, SRI LANKA*, THAILAND, VIETNAM

Salacistis rubicunda (Blume) M. C. Pace [New Combination]

B.: Neottia rubicunda Blume

PACE Matthew C., A Recircumscription of Goodyera (Orchidaceae), including the Description of Paorchis gen. nov., and Resurrection of Cionisaccus, Eucosia, and Salacistis. Brittonia 72 (3): 257-267. 2020 [266] doi: 10.1007/s12228-020-09623-y

CHINA (Taiwan, Yunnan); INDIA, INDONESIA*, JAPAN, MALAYSIA, PAPUA NEW GUINEA, PHILIPPINES, VIETNAM; [AUSTRALIA]

Spathoglottis arunachalensis Tsering \& K. Prasad [New Species] IUCN: CR: B1B2a,b(iii); Evidence: Morphology

TSERING Jambey, PRASAD Kothareddy, Spathoglottis arunachalensis (Orchidaceae), a New Species from Arunachal Pradesh, India. Phytotaxa 432 (3): 289-295. 2020 [289] doi: 10.11646/phytotaxa.432.3.6

CHINA (Xizang*)

Stereochilus arunachalensis Chowlu \& A. N. Rao [New Species] IUCN: NE; Evidence: Morphology

CHOWLU Krishna, RAO A. Nageswara, Stereochilus arunachalensis, a New Orchid Species of Orchidaceae (Epidendroideae: Vandeae:

Aeridinae) from Arunachal Pradesh, India. Phytotaxa 433 (2): 177-180. 2020 [177] doi: 10.11646/phytotaxa.433.2.10

CHINA (Xizang*)

Tainia acuminata Aver. 中越带唇兰 [New Record]

YUAN Quan, TAN Fei, QIN Ying, LIU Yan, Tainia acuminata, a Newly Recorded Species of Orchidaceae from China. Journal of Tropical and Subtropical Botany 28 (3): 245-247. 2020 [245] doi: 10.11926/jtsb.4168

CHINA (Guangxi); VIETNAM*

Vanda bicolor Griff. 双色万代兰 [New Recorded]

DENG Jie-ling, HUANG Chang-yan, CUI Xue-qiang, ZHANG Zi-bin, Vanda bicolor, A Newly Recorded Species of Vanda from China. Guihaia 40 (2): 282-284. 2020 [283] doi: 10.11931/guihaia.gxzw201811001

CHINA (Xizang); BHUTAN, INDIA, MYANMAR, NEPAL

\section{Orthotrichaceae 木灵蘚科}

Orthotrichum brassii E. B. Bartram [Excluded from China]

WANG Qing-hua, JIA Yu, A Taxonomic Study of the Genus Orthotrichum (Orthotrichaceae, Moss) in China. Acta Bryolichenologica Asiatica 9: 1-166. 2020 [147]

PAPUA NEW GUINEA

Orthotrichum erubescens Müll. Hal. [New Synomyms]

S.: Orthotrichum crispifolium Broth.; Orthotrichum griffithii Mitt. ex Dixon; Orthotrichum sinuosum Lewinsky

WANG Qing-hua, JIA Yu, A Taxonomic Study of the Genus Orthotrichum (Orthotrichaceae, Moss) in China. Acta Bryolichenologica Asiatica 9: 1-166. 2020 [64]

CHINA (Chongqing, Gansu, Hunan, Jiangxi, Shannxi, Sichuan, Xizang, Yunnan, Zhejiang); BHUTAN, INDIA, JAPAN

Orthotrichum pamiricum Plášek \& Sawicki [Excluded from China]

WANG Qing-hua, JIA Yu, A Taxonomic Study of the Genus Orthotrichum (Orthotrichaceae, Moss) in China. Acta Bryolichenologica Asiatica 9: 1-166. 2020 [150]

[ASIA]

Orthotrichum stramineum Hornsch. ex Brid. [New Synomyms] 
杜诚, 刘军, 叶文, 廖帅, 葛斌杰, 刘冰, 马金双 (2021) 中国植物新分类群、新名称 2020 年度报告. 生物多样性, 29, 10111020. http://www.biodiversity-science.net/CN/10.17520/biods.2021122

S.: Orthotrichum notabile Lewinsky-Haapasaari

WANG Qing-hua, JIA Yu, A Taxonomic Study of the Genus Orthotrichum (Orthotrichaceae, Moss) in China. Acta Bryolichenologica Asiatica 9: 1-166. 2020 [134]

CHINA (Gansu, Sichuan, Xizang); JAPAN, [EUROPE], [AMERICA]

\section{Orobanchaceae 列当科}

Pedicularis chumbica Prain [Typification]

GARG Arti, SINGH Rajeev Kumar, Typification of Fifty One Names in Pedicularis (Orobanchaceae). Phytotaxa 430 (2): $61-94.2020$ [79] doi: 10.11646/phytotaxa.430.2.1

CHINA (Xizang*); INDIA

Pedicularis confertiflora Prain [Typification]

GARG Arti, SINGH Rajeev Kumar, Typification of Fifty One Names in Pedicularis (Orobanchaceae). Phytotaxa 430 (2): $61-94.2020$ [80] doi: 10.11646/phytotaxa.430.2.1

CHINA (Xizang*); INDIA

Pedicularis daltonii Prain [Typification]

GARG Arti, SINGH Rajeev Kumar, Typification of Fifty One Names in Pedicularis (Orobanchaceae). Phytotaxa 430 (2): $61-94.2020$ [80] doi: 10.11646/phytotaxa.430.2.1

CHINA (Xizang); BHUTAN, INDIA*

Pedicularis daltonii Prain [Typification]

GARG Arti, SINGH Rajeev Kumar, Typification of Fifty One Names in Pedicularis (Orobanchaceae). Phytotaxa 430 (2): $61-94.2020$ [81] doi: 10.11646/phytotaxa.430.2.1

CHINA (Xizang); INDIA*

Pedicularis flexuosa Hook. f. [Typification]

GARG Arti, SINGH Rajeev Kumar, Typification of Fifty One Names in Pedicularis (Orobanchaceae). Phytotaxa 430 (2): $61-94.2020$ [82] doi: 10.11646/phytotaxa.430.2.1

CHINA (Xizang); BHUTAN, INDIA*, NEPAL

Pedicularis furfuracea Wall. ex Benth. [Typification]

GARG Arti, SINGH Rajeev Kumar, Typification of Fifty One Names in Pedicularis (Orobanchaceae). Phytotaxa 430 (2): $61-94.2020$

[82] doi: 10.11646/phytotaxa.430.2.1

CHINA (Xizang); BHUTAN, INDIA, NEPAL*

Pedicularis gracilis Wall. ex Benth. [Typification]

GARG Arti, SINGH Rajeev Kumar, Typification of Fifty One Names in Pedicularis (Orobanchaceae). Phytotaxa 430 (2): $61-94.2020$

[82] doi: 10.11646/phytotaxa.430.2.1

CHINA (Sichuan, Xizang. Yunnan); AFGHANISTAN, BHUTAN, INDIA, NEPAL*, PAKISTAN

Pedicularis hongii Kottaim. [New Name]

R.: Pedicularis multicaulis W. B. Yu, Hong Wang bis \& D. Z. Li

KOTTAIMUTHU Ramalingam, BASU Muthuramalingam Jothi, Pedicularis hongii Kottaim., a New Name for P. multicaulis W. B. Yu,

H. Wang \& D. Z. Li (Orobanchaceae). Annales Botanici Fennici 57 (4-6): 209-210. 2020 [209] doi: 10.5735/085.057.0403

CHINA (Yunnan*)

Pedicularis khoiyangii D. Borah \& R. Kr. Singh [New Species] IUCN: VU: D; Evidence: Morphology

BORAH Dipankar, GAP Neelam, SINGH Rajeev Kumar, Pedicularis khoiyangii (Orobanchaceae), a New Species from the Eastern Himalaya, India. Phytotaxa 430 (4): 287-293. 2020 [288] doi: 10.11646/phytotaxa.430.4.3

CHINA (Xizang*)

Pedicularis lachnoglossa Hook. f. [Typification]

GARG Arti, SINGH Rajeev Kumar, Typification of Fifty One Names in Pedicularis (Orobanchaceae). Phytotaxa 430 (2): $61-94.2020$ [86] doi: 10.11646/phytotaxa.430.2.1

CHINA (Sichuan, Xizang. Yunnan); BHUTAN, INDIA*, NEPAL

Pedicularis mollis Wall. ex Benth. [Typification]

GARG Arti, SINGH Rajeev Kumar, Typification of Fifty One Names in Pedicularis (Orobanchaceae). Phytotaxa 430 (2): $61-94.2020$ [86] doi: 10.11646/phytotaxa.430.2.1

CHINA (Xizang); BHUTAN, INDIA, NEPAL*

Pedicularis odontophora Prain [Typification] 
杜诚, 刘军, 叶文, 廖帅, 葛斌杰, 刘冰, 马金双 (2021) 中国植物新分类群、新名称 2020 年度报告. 生物多样性, 29, 10111020. http://www.biodiversity-science.net/CN/10.17520/biods.2021122

GARG Arti, SINGH Rajeev Kumar, Typification of Fifty One Names in Pedicularis (Orobanchaceae). Phytotaxa 430 (2): $61-94.2020$ [87] doi: 10.11646/phytotaxa.430.2.1

CHINA (Xizang); INDIA*

Pedicularis prainiana Maxim. [Typification]

GARG Arti, SINGH Rajeev Kumar, Typification of Fifty One Names in Pedicularis (Orobanchaceae). Phytotaxa 430 (2): $61-94.2020$ [88] doi: 10.11646/phytotaxa.430.2.1

CHINA (Xizang*)

Pedicularis rex C. B. Clarke ex Maxim. [Typification]

GARG Arti, SINGH Rajeev Kumar, Typification of Fifty One Names in Pedicularis (Orobanchaceae). Phytotaxa 430 (2): $61-94.2020$

[89] doi: 10.11646/phytotaxa.430.2.1

CHINA (Guizhou, Yunnan*)

Pedicularis robusta Hook. f. [Typification]

GARG Arti, SINGH Rajeev Kumar, Typification of Fifty One Names in Pedicularis (Orobanchaceae). Phytotaxa 430 (2): $61-94.2020$

[90] doi: 10.11646/phytotaxa.430.2.1

CHINA (Xizang); INDIA*

Pedicularis schizorrhyncha Prain [Typification]

GARG Arti, SINGH Rajeev Kumar, Typification of Fifty One Names in Pedicularis (Orobanchaceae). Phytotaxa 430 (2): $61-94.2020$ [90] doi: 10.11646/phytotaxa.430.2.1

CHINA (Xizang*)

Pedicularis siphonantha D. Don [Typification]

GARG Arti, SINGH Rajeev Kumar, Typification of Fifty One Names in Pedicularis (Orobanchaceae). Phytotaxa 430 (2): $61-94.2020$

[91] doi: 10.11646/phytotaxa.430.2.1

CHINA (Sichuan, Xizang, Yunnan); BHUTAN, INDIA, NEPAL*

Pedicularis tenuicaulis Prain [Typification]

GARG Arti, SINGH Rajeev Kumar, Typification of Fifty One Names in Pedicularis (Orobanchaceae). Phytotaxa 430 (2): $61-94.2020$

[91] doi: 10.11646/phytotaxa.430.2.1

CHINA (Xizang*)

Pedicularis trichoglossa Hook. f. [Typification]

GARG Arti, SINGH Rajeev Kumar, Typification of Fifty One Names in Pedicularis (Orobanchaceae). Phytotaxa 430 (2): $61-94.2020$

[91] doi: 10.11646/phytotaxa.430.2.1

CHINA (Sichuan, Xizang, Yunnan); BHUTAN, INDIA*, MYANMAR, NEPAL

Striga asiatica (L.) Kuntze [Typification]

SINGH Rajeev Kumar, New Lectotypification of the Linnaean Name Buchnera asiatica (Orobanchaceae). Kew Bulletin 75 (23): 1-3. 2020 [3] doi: 10.1007/S12225-020-9872-8

CHINA (Fujian, Guangdong, Guangxi, Guizhou, Hunan, Jiangxi, Taiwan, Yunnan); BHUTAN, CAMBODIA, INDIA, NEPAL, PHILIPPINES, SRI LANKA, THAILAND, VIETNAM; [AFRICA]; [AMERICA]

\section{Oxalidaceae 酢浆草科}

Oxalis articulata Savigny 关节酢浆草 [Correction]

A.: Oxalis martiana auct. non. Zucc.: Z. Wei \& Y. B. Zhang in Z. Wei \& Y. Q. He, Fl. Zhejiang 3: 399. 1993

CHEN Feng, XIE Wen-yuan, ZHANG Shui-li, ZHANG Fen-yao, CHEN Zheng-hai, Additional Notes on the Seed Plants in Zhejiang (II). Journal of Hangzhou Normal University (Natural Science Edition) 19 (6): 96-102. 2020 [98] doi: 10.12191/j.issn.1674232X.2020.06.014

CHINA (Zhejiang)

Oxalis obtriangulata Maxim. 三角叶酢浆草 [Correction]

A.: Oxalis acetosella L. subsp. japonica auct. non. H. Hara: C. C. Huang \& al. in L. R. Xu \& C. C. Huang, Fl. Reipubl. Popularis Sin. 43 (1): 9.1998

CHEN Feng, XIE Wen-yuan, ZHANG Shui-li, ZHANG Fen-yao, CHEN Zheng-hai, Additional Notes on the Seed Plants in Zhejiang (II). Journal of Hangzhou Normal University (Natural Science Edition) 19 (6): 96-102. 2020 [97] doi: 10.12191/j.issn.1674232X.2020.06.014

CHINA (Zhejiang)

Oxalis shibeishanensis Huan C. Wang \& Y. Tian 石碑山酢浆草 [New Species] IUCN: NE; Evidence: Morphology 
杜诚, 刘军, 叶文, 廖帅, 葛斌杰, 刘冰, 马金双 (2021) 中国植物新分类群、新名称 2020 年度报告. 生物多样性, 29, 10111020. http://www.biodiversity-science.net/CN/10.17520/biods.2021122

TIAN Ye, YANG Feng, LIU Xiao-lan, WANG Huan-chong, Oxalis shibeishanensis (Oxalidaceae), a New Species from Yunnan, Southwest China. Taiwania 65 (3): 360-363. 2020 [354] doi: 10.6165/tai.2020.65.360

CHINA (Yunnan*)

\section{Papaveraceae 罈科}

Corydalis paeoniifolia (Steph.) Pers. 苟叶紫堇 [New Record]

LI Shi-feng, ZHAO Jian-xin, ZHOU Ming, GAO Jian-zhi, JIN Dong-qiang, CHEN Guan-Jun, GONG Kui-lin, A New Record of Corydalis from China - Corydalis paeoniifolia (Steph.) Pers. Inner Mongolia Forestry Investigation and Design 43 (1): 32-34. 2020 [32] doi: 10.13387/j.cnki.nmld.2020.01.014

CHINA (Nei Mongol); MONGOLIA, RUSSIA

Ichtyoselmis macrantha (Oliver) Lidén subsp. porphyrantha J. Y. Shen \& Q. B. Gong 紫花黄药 [New Subspecies]

GONG Qiang-bang, MA Xing-da, WANG Wen-guang, SHI Ji-pu, YIN Hai-jun, DUAN Chun-cheng, SHEN Jian-yong, Ichtyoselmis macrantha subsp. porphyrantha (Papaveraceae), a New Subspecies from West Yunnan and North Myanmar. Taiwania 65 (3): $423-$ 425. 2020 [423] doi: 10.6165/tai.2020.65.423

CHINA (Yunnan*); MYANMAR

\section{Pentaphylacaceae 五列木科}

Cleyera japonica Thunb. subsp. pleiopetala Z. H. Chen, P. L. Chiu \& G. Y. Li 多瓣杨桐 [New Subspecies]

XIE Wen-yuan, CHEN Feng, ZHANG Fen-yao, XU Shao-qing, CHEN Zheng-hai, Correction and Supplement of the Seed Plant Flora in Zhejiang. Journal of Hangzhou Normal University (Natural Science Edition) 19 (3): 247-252. 2020 [250] doi: 10.12191/j.issn.1674-232X.2020.03.005

CHINA (Zhejiang*)

Eurya aureopunctata (Hung T. Chang) Z. H. Chen \& P. L. Chiu 黄腺柃 [New Combination]

B.: Eurya loquaiana Dunn var. aureopunctata Hung T. Chang

XIE Wen-yuan, CHEN Feng, ZHANG Fen-yao, XU Shao-qing, CHEN Zheng-hai, Correction and Supplement of the Seed Plant Flora in Zhejiang. Journal of Hangzhou Normal University (Natural Science Edition) 19 (3): 247-252. 2020 [249] doi: 10.12191/j.issn.1674-232X.2020.03.005

CHINA (Guangdong*, Guangxi, Yunnan, Zhejiang)

\section{Phyllanthaceae 叶下珠科}

Bridelia tomentosa Blume [New Synonyms]

S.: Berchemia sessiliflora Benth.

DENG Yun-fei, Berchemia sessiliflora (Rhamnaceae) Is Bridelia tomentosa (Phyllanthaceae). Phytotaxa 442 (2): 128-132. 2020 [131] doi: 10.11646/phytotaxa.442.2.8

CHINA (Hong Kong); INDONESIA*

Glochidion ellipticum Wight [New Synonyms]

S.: Glochidion ovalifolium F. Y. Lu \& Y. S. Hsu

YAO Gang, SONG Zhu-qiu, XUE Bin-e, SHI Shi, LI Yu-ling, LUO Shi-xiao, Taxonomic Revision of the Genus Glochidion (Phyllanthaceae) in Taiwan, China. PhytoKeys 159: 137-159. 2020 [143] doi: 10.3897/phytokeys.159.54839

CHINA (Taiwan); INDIA*

Glochidion lanceolatum Hayata [New Synonyms]

S.: Glochidion kusukusense Hayata

YAO Gang, SONG Zhu-qiu, XUE Bin-e, SHI Shi, LI Yu-ling, LUO Shi-xiao, Taxonomic Revision of the Genus Glochidion (Phyllanthaceae) in Taiwan, China. PhytoKeys 159: 137-159. 2020 [144] doi: 10.3897/phytokeys.159.54839

CHINA (Taiwan*)

Glochidion lanceolatum Hayata [Typification]

YAO Gang, SONG Zhu-qiu, XUE Bin-e, SHI Shi, LI Yu-ling, LUO Shi-xiao, Taxonomic Revision of the Genus Glochidion (Phyllanthaceae) in Taiwan, China. PhytoKeys 159: 137-159. 2020 [144] doi: 10.3897/phytokeys.159.54839

CHINA (Taiwan*)

Glochidion lanyuense Gang Yao \& S. X. Luo [New Species] IUCN: NE; Evidence: Morphology

YAO Gang, SONG Zhu-qiu, XUE Bin-e, SHI Shi, LI Yu-ling, LUO Shi-xiao, Taxonomic Revision of the Genus Glochidion (Phyllanthaceae) in Taiwan, China. PhytoKeys 159: 137-159. 2020 [147] doi: 10.3897/phytokeys.159.54839

CHINA (Taiwan*)

Glochidion rubrum Blume [New Synonyms] 
杜诚, 刘军, 叶文, 廖帅, 葛斌杰, 刘冰, 马金双 (2021) 中国植物新分类群、新名称 2020 年度报告. 生物多样性, 29, 10111020. http://www.biodiversity-science.net/CN/10.17520/biods.2021122

S.: Glochidion chademenosocarpum Hayata

YAO Gang, SONG Zhu-qiu, XUE Bin-e, SHI Shi, LI Yu-ling, LUO Shi-xiao, Taxonomic Revision of the Genus Glochidion (Phyllanthaceae) in Taiwan, China. PhytoKeys 159: 137-159. 2020 [152] doi: 10.3897/phytokeys.159.54839

CHINA (Taiwan); INDONESIA*

Glochidion lanceolatum Hayata [Typification]

YAO Gang, SONG Zhu-qiu, XUE Bin-e, SHI Shi, LI Yu-ling, LUO Shi-xiao, Taxonomic Revision of the Genus Glochidion (Phyllanthaceae) in Taiwan, China. PhytoKeys 160: 1-6. 2020 [152] doi: 10.3897/phytokeys.160.54459

CHINA (Taiwan*)

\section{Phytolaccaceae 商陆科}

Phytolacca americana L. var. huadongensis X. H. Li 华东商陆 [New Variety]

LI Xin-hua, YIN Xiao-ming, Phytolacca americana var. huadongensis (Phytolaccaceae), a New Chinese Variety with Thyrsoid Inflorescences. Phytotaxa 475 (2): 109-116. 2020 [112] doi: 10.11646/phytotaxa.475.2.5

CHINA (Anhui, Jiangsu*)

Phytolacca yunnanensis X. H. Li \& Wen Zhou [New Species] IUCN: NE; Evidence: Morphology

LI Xin-hua, ZHOU Wen, GUO Jia-cheng, YIN Xiao-ming, Phytolacca yunnanensis (Phytolaccaceae), a New Species from China with Distinctive Inflorescence Characteristics. Phytotaxa 446 (1): 49-54. 2020 [51] doi: 10.11646/phytotaxa.446.1.6

CHINA (Yunnan*)

\section{Pinaceae 松科}

Abies fanjingshanensis W. L. Huang, Y. L. Tu \& S. Z. Fang 梵净山冷杉 [Typification]

YANG Yong, RUSHFORTH Keith, Lectotypification of Abies fanjingshanensis (Pinaceae). PhytoKeys 152: 105-110. 2020 [109] doi: 10.3897/phytokeys.152.51494

CHINA (Guizhou*)

\section{Piperaceae 胡椒科}

Peperomia japonica Makino [Typification]

LU Yu-chen, YANG Tsung-yu Aleck, Taxonomy of Peperomia (Piperaceae) in Taiwan. Taiwania 65 (4): 500-516. 2020 [511] doi: 10.6165/tai.2020.65.500

CHINA (Fujian, Taiwan); JAPAN*

Piper lanyuense K. N. Kung \& Kun C. Chang [New Species] IUCN: NE; Evidence: Morphology

CHANG Kun-cheng, KUNG Kuan-ning, Piper lanyuense (Piperaceae), a New Species from Taiwan. Annales Botanici Fennici 57 (13): 93-96. 2020 [93] doi: 10.5735/085.057.0113

CHINA (Taiwan*)

Piper semi-transparens C. Y. Hao \& Y. H. Tan [New Species] IUCN: NE; Evidence: ITS; Morphology

HAO Chao-yun, WU Bao-duo, QIN Xiao-wei, WU Gang, DENG Hai-yan, TAN Yun-hong, Morphological and Molecular Evidence for a New Species from China: Piper semitransparens (Piperaceae). Phytotaxa 429 (3): 191-199. 2020 [194] doi: 10.11646/phytotaxa.429.3.1

CHINA (Hainan*)

\section{Plagiochilaceae 羽苔科}

Plagiochila xerophila Bakalin \& Vilnet [New Species] IUCN: NE; Evidence: Morphology

BAKALIN Vadim, VILNET Anna, Plagiochila xerophila (Plagiochilaceae, Marchantiophyta) - a Highly Xerophilous New Species from the Tibetan Spur (China). Plant Ecology and Evolution 153 (1): 120-131. 2020 [127] doi: 10.5091/plecevo.2020.1560

CHINA (Sichuan*)

\section{Poaceae 禾本科}

Calamagrostis hongii Paszko \& Bing Liu 洪氏野青茅 [New Species] IUCN: NE; Evidence: Morphology

LIU Bing, PASZKO Beata, Calamagrostis hongii (Poaceae, Agrostidinae), a New Species from Southwestern China. PhytoKeys 166: 41-55. 2020 [43] doi: 10.3897/phytokeys.166.53010

CHINA (Chongqing, Guizhou, Sichuan, Xizang, Yunnan*)

Chloris pilosa Schumach. 毛虎尾草 [New Record] 
杜诚, 刘军, 叶文, 廖帅, 葛斌杰, 刘冰, 马金双 (2021) 中国植物新分类群、新名称 2020 年度报告. 生物多样性, 29, 10111020. http://www.biodiversity-science.net/CN/10.17520/biods.2021122

LIAO Hsien-chun, CHAN Ming-hui, CHEN Chih-hui, Barcode of Nuclear Ribosomal Internal Transcribed Spacer Regions (ITS) as a Useful Tool to Recognize a Newly Naturalized and Potentially Invasive Weed, Chloris pilosa Schumach. (Poaceae), in Taiwan. Taiwania 65 (2): 129-139. 2020 [137] doi: 10.6165/tai.2020.65.129

CHINA (Taiwan); [AFRICA]

Dendrocalamus latiflorus Munro var. rongshuiensis D. Y. Huang \& al. 融水麻竹 [New Variety]

HUANG Da-yong, LI Li-jie, XU Zhen-guo, ZHOU Tong, LU Zong-jun, LUO Dong-sheng, A New Variety of Dendrocalamus: D. latiflorus var. rongshuiensis. World Bamboo and Rattan 18 (5): 62-63. 2020 [62] doi: 10.12168/sjzttx.2020.05.012

CHINA (Guangxi)

Eriochloa contracta Hitchc. 扁野乘 [New Record]

JUNG Ming-jer, Three Newly Naturalized Eriochloa Kunth Grasses (Poaceae) in Taiwan. Taiwan Quarterly Journal of Forest Research 42 (4): 215-228. 2020 [216]

CHINA (Taiwan); UNITED STATES

Eriochloa punctata (L.) Desv. ex Ham. 苍白野㯟 [New Record]

JUNG Ming-jer, Three Newly Naturalized Eriochloa Kunth Grasses (Poaceae) in Taiwan. Taiwan Quarterly Journal of Forest Research 42 (4): 215-228. 2020 [216]

CHINA (Taiwan); JAMAICA*

Eriochloa succincta (Trin.) Kunth 伏生野㯟 [New Record]

JUNG Ming-jer, Three Newly Naturalized Eriochloa Kunth Grasses (Poaceae) in Taiwan. Taiwan Quarterly Journal of Forest Research 42 (4): 215-228. 2020 [218]

CHINA (Taiwan); RUSSIA*

Fargesia purpurea D. Z. Li \& Xia Y. Ye 紫鞘箭竹 [New Species] IUCN: NE; Evidence: Morphology

YE Xia-ying, ZHANG Yu-xiao, LI De-zhu, Two New Species of Fargesia (Poaceae, Bambusoideae) from Southwestern China. PhytoKeys 170: 25-37. 2020 [29] doi: 10.3897/phytokeys.170.58780

CHINA (Xizang*)

Fargesia viridis D. Z. Li \& Xia Y. Ye 翠绿箭竹 [New Species] IUCN: NE; Evidence: Morphology

YE Xia-ying, ZHANG Yu-xiao, LI De-zhu, Two New Species of Fargesia (Poaceae, Bambusoideae) from Southwestern China. PhytoKeys 170: 25-37. 2020 [27] doi: 10.3897/phytokeys.170.58780

CHINA (Yunnan*)

Garnotia emodi (Arn. \& Nees) Janowski 喜马拉雅耳稃草 [New Record]

LIU Cheng, YA Ji-dong, GUO Yong-jie, CAI Jie, ZHANG Ting, Newly recorded species of seed plants from Xizang, China. Biodiversity Science 28 (10): 1238-1245. 2020 [1239] doi: 10.17520/biods.2020161

CHINA (Xizang); INDIA*

Ravenochloa D. Z. Li \& Y. X. Zhang 雷文竹属 [New Genus]

ZHANG Yu-xiao, GUO Cen, LI De-zhu, A New Subtribal Classification of Arundinarieae (Poaceae, Bambusoideae) with the Description of a New Genus. Plant Diversity 42 (3): 127-134. 2020 [132] doi: 10.1016/j.pld.2020.03.004 CHINA

Ravenochloa wilsonii (Rendle) D. Z. Li \& Y. X. Zhang 雷文竹 [New Combination]

B.: Arundinaria wilsonii Rendle

ZHANG Yu-xiao, GUO Cen, LI De-zhu, A New Subtribal Classification of Arundinarieae (Poaceae, Bambusoideae) with the Description of a New Genus. Plant Diversity 42 (3): 127-134. 2020 [132] doi: 10.1016/j.pld.2020.03.004

CHINA (Chongqing, Huibei*)

Sinosasa L. C. Chia ex N. H. Xia, Q. M. Qin \& Y. H. Tong 华赤竹属 [New Genus]

QIN Qiao-mei, TONG Yi-hua, ZHENG Xi-rong, NI Jing-bo, XIA Nian-he, Sinosasa (Poaceae: Bambusoideae), a New Genus from China. Taxon 70 (1): 27-47. 2020 [31] doi: 10.1002/tax.12422 CHINA

Sinosasa fanjingshanensis N. H. Xia, Q. M. Qin \& J. B. Ni 梵净山华赤竹 [New Species] GBSSI, atpI-H, psaA-ORF170, rpl32-trnL, rpoB-trnC, rps16-trnQ, trnD-T, trnS-G, trnT-L; Morphology

QIN Qiao-mei, TONG Yi-hua, ZHENG Xi-rong, NI Jing-bo, XIA Nian-he, Sinosasa (Poaceae: Bambusoideae), a New Genus from China. Taxon 70 (1): 27-47. 2020 [34] doi: 10.1002/tax.12422

CHINA (Guizhou)

Sinosasa guangxiensis (C. D. Chu \& C. S. Chao) N. H. Xia, Q. M. Qin \& X. R. Zheng 广西华赤竹 [New Combination] 
杜诚, 刘军, 叶文, 廖帅, 葛斌杰, 刘冰, 马金双 (2021) 中国植物新分类群、新名称 2020 年度报告. 生物多样性, 29, 10111020. http://www.biodiversity-science.net/CN/10.17520/biods.2021122

B.: Sasa guangxiensis C. D. Chu \& C. S. Chao

QIN Qiao-mei, TONG Yi-hua, ZHENG Xi-rong, NI Jing-bo, XIA Nian-he, Sinosasa (Poaceae: Bambusoideae), a New Genus from China. Taxon 70 (1): 27-47. 2020 [34] doi: 10.1002/tax.12422

CHINA (Guangxi)

Sinosasa huapingensis N. H. Xia, Q. M. Qin \& Y. H. Tong 花坪华赤竹 [New Species] GBSSI, atpI-H, psaA-ORF170, rpl32-trnL, rpoB-trnC, rps16-trnQ, trnD-T, trnS-G, trnT-L; Morphology

QIN Qiao-mei, TONG Yi-hua, ZHENG Xi-rong, NI Jing-bo, XIA Nian-he, Sinosasa (Poaceae: Bambusoideae), a New Genus from China. Taxon 70 (1): 27-47. 2020 [39] doi: 10.1002/tax.12422

CHINA (Guangxi)

Sinosasa longiligulata (McClure) N. H. Xia, Q. M. Qin \& J. B. Ni 华赤竹 [New Combination]

B.: Sasa longiligulata McClure

QIN Qiao-mei, TONG Yi-hua, ZHENG Xi-rong, NI Jing-bo, XIA Nian-he, Sinosasa (Poaceae: Bambusoideae), a New Genus from China. Taxon 70 (1): 27-47. 2020 [33] doi: 10.1002/tax.12422

CHINA (Guangdong)

Sinosasa magninoda (T. H. Wen \& G. L. Liao) N. H. Xia, Q. M. Qin \& X. R. Zheng 大节华赤竹 [New Combination]

B.: Sasa magninoda T. H. Wen \& G. L. Liao

QIN Qiao-mei, TONG Yi-hua, ZHENG Xi-rong, NI Jing-bo, XIA Nian-he, Sinosasa (Poaceae: Bambusoideae), a New Genus from China. Taxon 70 (1): 27-47. 2020 [41] doi: 10.1002/tax.12422

CHINA (Jiangxi)

Sinosasa mingyueshanensis N. H. Xia, Q. M. Qin \& X. R. Zheng 明月山华赤竹 [New Species] GBSSI, atpI-H, psaA-ORF170, rpl32trnL, rpoB-trnC, rps16-trnQ, trnD-T, trnS-G, trnT-L; Morphology

QIN Qiao-mei, TONG Yi-hua, ZHENG Xi-rong, NI Jing-bo, XIA Nian-he, Sinosasa (Poaceae: Bambusoideae), a New Genus from China. Taxon 70 (1): 27-47. 2020 [42] doi: 10.1002/tax.12422

CHINA (Jiangxi)

Sinosasa polytricha N. H. Xia, Q. M. Qin \& X. R. Zheng 多毛华赤竹 [New Species] GBSSI, atpI-H, psaA-ORF170, rpl32-trnL, rpoB-trnC, rps16-trnQ, trnD-T, trnS-G, trnT-L; Morphology

QIN Qiao-mei, TONG Yi-hua, ZHENG Xi-rong, NI Jing-bo, XIA Nian-he, Sinosasa (Poaceae: Bambusoideae), a New Genus from China. Taxon 70 (1): 27-47. 2020 [43] doi: 10.1002/tax.12422

CHINA (Guangdong, Hunan)

Stipa $\times$ consanguinea Trin. ex Bunge [Typification]

NOBIS Marcin, GUDKOVA Polina D., NOWAK Arkadiusz, SAWICKI Jakub, NOBIS Agnieszka, A Synopsis of the Genus Stipa (Poaceae) in Middle Asia, Including a Key to Species Identification, an Annotated Checklist, and Phytogeographic Analyses. Annals of the Missouri Botanical Garden 105 (1): 1-63. 2020 [24] doi: 10.3417/2019378

CHINA (Xinjiang); MONGOLIA, RUSSIA

Stipa bungeana Trin. ex Bunge [Typification]

NOBIS Marcin, GUDKOVA Polina D., NOWAK Arkadiusz, SAWICKI Jakub, NOBIS Agnieszka, A Synopsis of the Genus Stipa (Poaceae) in Middle Asia, Including A Key to Species Identification, an Annotated Checklist, and Phytogeographic Analyses. Annals of the Missouri Botanical Garden 105 (1): 1-63. 2020 [18] doi: 10.3417/2019378

CHINA (Anhui, Gansu, Hebei, Henan, Jiangsu, Nei Mongol, Ningxia, Qinghai, Shaanxi, Shandong, Sichuan, Xinjiang, Xizang); KAZAKHSTAN, KYRGYZSTAN, MONGOLIA, PAKISTAN

Stipa richteriana Kar. \& Kir. [Typification]

NOBIS Marcin, GUDKOVA Polina D., NOWAK Arkadiusz, SAWICKI Jakub, NOBIS Agnieszka, A Synopsis of the Genus Stipa (Poaceae) in Middle Asia, Including a Key to Species Identification, an Annotated Checklist, and Phytogeographic Analyses. Annals of the Missouri Botanical Garden 105 (1): 1-63. 2020 [43] doi: 10.3417/2019378

CHINA (Xinjiang); KAZAKHSTAN, UZBEKISTAN

Stipa sareptana A. K. Becker [Typification]

NOBIS Marcin, GUDKOVA Polina D., NOWAK Arkadiusz, SAWICKI Jakub, NOBIS Agnieszka, A Synopsis of the Genus Stipa (Poaceae) in Middle Asia, Including a Key to Species Identification, an Annotated Checklist, and Phytogeographic Analyses. Annals of the Missouri Botanical Garden 105 (1): 1-63. 2020 [45] doi: 10.3417/2019378

CHINA (Gansu, Hebei, Nei Mongol, Ningxia, Qinghai, Shanxi, Xinjiang, Xizang); KAZAKHSTAN, MONGOLIA, RUSSIA, TAJIKISTAN

Tripidium rufipilum (Steud.) Welker, Voronts. \& E. A. Kellogg [New Combination]

B.: Saccharum rufipilum Steud. 
杜诚, 刘军, 叶文, 廖帅, 葛斌杰, 刘冰, 马金双 (2021) 中国植物新分类群、新名称 2020 年度报告. 生物多样性, 29, 10111020. http://www.biodiversity-science.net/CN/10.17520/biods.2021122

WELKER Cassiano A. Dorneles, VORONTSOVA Maria S., KELLOGG Elizabeth A., A New Combination in the Genus Tripidium (Poaceae: Andropogoneae). Phytotaxa 471 (3): 297-300. 2020 [297] doi: 10.11646/phytotaxa.471.3.12

CHINA (Gansu, Shaanxi, Sichuan, Xizang, Yunnan); BHUTAN, INDIA, MYANMAR, NEPAL*, PAKISTAN

\section{Polygonaceae 苶科}

Polygonum nepalense Meisn. 尼泊尔苶 [New Synonyms]

S.: Polygonum minutissimum Z. Wei \& Y. B. Chang nom. illeg.

CHEN Feng, XIE Wen-yuan, ZHANG Fen-yao, ZHANG Hong-wei, CHEN Xian-xing, CHEN Zheng-hai, Notes on Seed Plant in Zhejiang Province (V). Journal of Zhejiang Forestry Science and Technology 40 (4): 67-72. 2020 [68] doi: 10.3969/j.issn.10013776.2020.04.012

CHINA (Zhejiang); NEPAL*

\section{Polypodiaceae 水龙骨科}

$\times$ Ellipisorus Li Bing Zhang \& Liang Zhang [New Name]

R.: $\times$ Lepinema Li Bing Zhang \& Liang Zhang

ZHANG Liang, ZHANG Li-bing, $\times$ Ellipisorus nom. nov. for $\times$ Lepinema (Polypodiaceae) and $\times$ Ellipisorus inaequibasis com. nov. from China. Phytotaxa 464 (4): 299-300. 2020 [299] doi: 10.11646/phytotaxa.464.4.4

CHINA

$\times$ Ellipisorus inaequibasis (Ching \& S. K. Wu) Li Bing Zhang \& Liang Zhang [New Combination]

B.: Platygyria $\times$ inaequibasis Ching \& S. K. Wu

ZHANG Liang, ZHANG Li-bing, $\times$ Ellipisorus nom. nov. for $\times$ Lepinema (Polypodiaceae) and $\times$ Ellipisorus inaequibasis com. nov. from China. Phytotaxa 464 (4): 299-300. 2020 [299] doi: 10.11646/phytotaxa.464.4.4

CHINA (Sichuan, Xizang, Yunnan*)

$\times$ Ellipisorus inaequibasis (Ching \& S. K. Wu) Li Bing Zhang \& Liang Zhang [New Synonyms]

S.: $\times$ Lepinema inaequibasis (Ching \& S. K. Wu) Li Bing Zhang \& Liang Zhang

ZHANG Liang, ZHANG Li-bing, $\times$ Ellipisorus nom. nov. for $\times$ Lepinema (Polypodiaceae) and $\times$ Ellipisorus inaequibasis com. nov. from China. Phytotaxa 464 (4): 299-300. 2020 [299] doi: 10.11646/phytotaxa.464.4.4

CHINA (Sichuan, Xizang, Yunnan*)

$\times$ Lepinema Li Bing Zhang \& Liang Zhang [New Genus]

H.: Lepisorus (J. Sm.) Ching $\times$ Ellipinema Li Bing Zhang \& Liang Zhang

ZHANG Liang, ZHANG Li-bing, Fern nothogenera and $\times$ Lepinema, a New Hybrid Genus between Ellipinema and Lepisorus (Polypodiaceae). Phytotaxa 455 (4): 262-266. 2020 [264] doi: 10.11646/phytotaxa.455.4.3 CHINA

$\times$ Lepinema inaequibasis (Ching \& S. K. Wu) Li Bing Zhang \& Liang Zhang [New Combination]

B.: Platygyria $\times$ inaequibasis Ching \& S. K. Wu

ZHANG Liang, ZHANG Li-bing, Fern nothogenera and $\times$ Lepinema, a New Hybrid Genus between Ellipinema and Lepisorus (Polypodiaceae). Phytotaxa 455 (4): 262-266. 2020 [264] doi: 10.11646/phytotaxa.455.4.3

CHINA (Sichuan, Xizang, Yunnan*)

Ellipinema Li Bing Zhang \& Liang Zhang [New Genus]

ZHANG Liang, ZHOU Xin-mao, LIANG Zhen-long, FAN Xue-ping, LUE Ngan Thi, SONG Min-shu, KNAPP Ralf, GAO Xin-fen, SUN Hang, ZHANG Li-bing, Phylogeny and Classification of the Tribe Lepisoreae (Polypodiaceae; Pteridophyta) with the Description of a New Genus, Ellipinema gen. nov., Segregated from Lepisorus. Molecular Phylogenetics and Evolution 148 (106803): 1-11. 2020 [6] doi: 10.1016/j.ympev.2020.106803

CHINA

Ellipinema jakonense (Blanf.) Li Bing Zhang \& Liang Zhang [New Combination]

B.: Polypodium clathratum C. B. Clarke var. jakonense Blanf.

ZHANG Liang, ZHOU Xin-mao, LIANG Zhen-long, FAN Xue-ping, LUE Ngan Thi, SONG Min-shu, KNAPP Ralf, GAO Xin-fen, SUN Hang, ZHANG Li-bing, Phylogeny and Classification of the Tribe Lepisoreae (Polypodiaceae; Pteridophyta) with the Description of a New Genus, Ellipinema gen. nov., Segregated from Lepisorus. Molecular Phylogenetics and Evolution 148 (106803): 1-11. 2020 [6] doi: 10.1016/j.ympev.2020.106803

CHINA (Xizang); INDIA*

Lepisorus youxingii R. H. Jiang \& X. C. Zhang [New Species] IUCN: LC; Evidence: rbcL, matK, psbZ, rbcL-atpB, rps4-trnS, trnL-F, rpl32-trnP, trnT; Morphology

JIANG Ri-hong, WANG Ya-rong, ZHANG Xian-chun, Lepisorus youxingii (Polypodiaceae), a New Fern Species from Guangxi, China. Phytotaxa 437 (2): 97-104. 2020 [102] doi: 10.11646/phytotaxa.437.2.5 
杜诚, 刘军, 叶文, 廖帅, 葛斌杰, 刘冰, 马金双 (2021) 中国植物新分类群、新名称 2020 年度报告. 生物多样性, 29, 10111020. http://www.biodiversity-science.net/CN/10.17520/biods.2021122

CHINA (Guangxi*)

Leptochilus gracilis Z. L. Liang, Liang Zhang \& Li Bing Zhang [New Species] IUCN: CR; Evidence: rps4, rps4-trnS, trnL, trnL-F; Morphology

LIANG Zhen-long, SHUI Yu-min, CHEN Wen-hong, YU Zhi-yong, ZHANG Liang, ZHANG Li-bing, Leptochilus gracilis (Polypodiaceae), a New Fern Species from Southeastern Yunnan, China Based on Morphological and Molecular Evidence. Phytotaxa 468 (1): 112-120. 2020 [117] doi: 10.11646/phytotaxa.468.1.7

CHINA (Yunnan*)

Selliguea shandongensis (Jian X. Li \& C. Y. Wang) Jian X. Li \& Xiao J. Li 山东假瘤蕨 [New Combination]

B.: Phymatopteris shandongensis Jian X. Li \& C. Y. Wang

LI Xiao-juan, LI Jian-xiu, Palynology of Polypodiaceae from Shandong and Its Significance in Classification. Guihaia 40 (4): $443-$ 451. 2020 [450] doi: 10.11931/guihaia.gxzw2019 03048

CHINA (Shandong*)

\section{Porellaceae 光蕶苔科}

Porella pinnata L. [Excluded from China]

BAI Yang, QIAN Kai, XIANG You-liang, YANG Zhi-wang, MAMTIMIN Sulayman, LI Wei, SHU Lei, ZHU Rui-liang, Porella pinnata (Porellaceae) excluded from the Liverwort Flora of Asia. Nova Hedwigia 150: 193-200. 2020 [193] doi: 10.1127/novasuppl/2020/193

[AMERICA]

\section{Primulaceae 报春花科}

Androsace coccinea Franch. [Typification]

XU Yuan, HU Chi-ming, HAO Gang, Proposal to Conserve the Name Androsace bulleyana Against A. coccinea (Primulaceae). Taxon 69 (4): 830-831. 2020 [830] doi: 10.1002/tax.12306

CHINA (Yunnan*)

Lysimachia daqiaoensis G. D. Tang \& R. Z. Huang [New Species] IUCN: CR; Evidence: Morphology

HUANG Rui-zhou, LIAO Miao, HAN Wei, YANG Yuan-zhi, ZHOU Ming-yi, FENG Han-hua, TANG Guang-da, Lysimachia daqiaoensis (Primulaceae), a New Cave Species from Guangdong, China. Phytotaxa 430 (1): 41-45. 2020 [41] doi: 10.11646/phytotaxa.430.1.6

CHINA (Guangdong*)

Lysimachia porcatisepala S. R. Yi [New Species] IUCN: CR; Evidence: Morphology

Yi Si-rong, Lysimachia porcatisepala, a New Species of Lysimachia (Primulaceae) from Chongqing, China. Phytotaxa 434 (1): $118-$ 122. 2020 [118] doi: 10.11646/phytotaxa.434.1.9

CHINA (Chongqing*)

Lysimachia xiangxiensis D. G. Zhang, C. Mou \& Yu Wu [New Species] IUCN: DD; Evidence: ITS; Morphology

MOU Cun, WU Yu, XIANG Liang, XIANG Xiao-mei, ZHANG Dai-gui, Lysimachia xiangxiensis (Primulaceae), a New Species from Limestone Area in Hunan Province, Central China. PhytoKeys 140: 23-32. 2020 [27] doi: 10.3897/phytokeys.140.47995

CHINA (Hunan*)

Primula qiupuensis J. W. Shao [New Species] IUCN: VU; Evidence: Morphology

ZHANG Chao, ZHANG Jian-ye, CHEN Fang-ming, CHEN Wen-hao, CHEN Dong-sheng, SHAO Jian-wen, Primula qiupuensis, a New Species in Primulaceae from China. Phytotaxa 441 (2): 176-182. 2020 [177] doi: 10.11646/phytotaxa.441.2.4

CHINA (Anhui*)

Primula sinensis Sabine ex Lindl. [Typification]

Xu Yuan, Deng Yun-fei, Hao Gang, Hu Chi-ming, Proposal to Conserve the Name Primula sinensis Sabine ex Lindl. Against P. sinensis Lour. (Primulaceae). Taxon 69 (4): 831-832. 2020 [831] doi: 10.1002/tax.12307

CHINA (Guangdong*)

Primula sunhangii T. Deng, D. G. Zhang \& Jiao Sun 神农架报春 [New Species] IUCN: CR: B2abiii; Evidence: ITS, rbcL, matK; Morphology

SUN Jiao, ZHANG Dai-gui, HUANG Xian-han, TOJIBAEV Komiljon, YANG Jing-yuan, WANG Heng-chang, DENG Tao, Primula sunhangii (Primulaceae): a New Species from Hubei, Central China. PhytoKeys 156: 103-112. 2020 [106] doi: 10.3897/phytokeys.156.49137

CHINA (Hubei*)

\section{Pteridaceae 凤尾蕨科}


杜诚, 刘军, 叶文, 廖帅, 葛斌杰, 刘冰, 马金双 (2021) 中国植物新分类群、新名称 2020 年度报告. 生物多样性, 29, 10111020. http://www.biodiversity-science.net/CN/10.17520/biods.2021122

Ceratopteris shingii Y. H. Yan \& Rui Zhang bis [New Species] IUCN: VU; Evidence: rbcL, trnL-F, psbCtrnS, trnW-P, rbcL-atpB; Chromosome; Morphology

ZHANG Rui, YU Jun-hao, SHAO Wen, WANG Wei-qing, SHANG Hui, ZHENG Xi-long, YAN Yue-hong, Ceratopteris shingii, a New Species of Ceratopteris with Creeping Rhizomes from Hainan, China. Phytotaxa 449 (1): 23-30. 2020 [24] doi: 10.11646/phytotaxa.449.1.3

CHINA (Hainan*)

\section{Ranunculaceae 毛茛科}

Aconitum delavayi Franch. [Typification]

KUMAR Anand, GHOSHAL Partha Pratim, BHARATI Kumar Avinash, Notes on the Type Specimens of Names in Aconitum (Ranunculaceae) at Central National Herbarium (CAL). Phytotaxa 475 (4): 209-238. 2020 [211] doi: 10.11646/phytotaxa.475.4.1 CHINA (Yunnan*)

Aconitum ferox Wall. ex Ser. var. flavidiflora Brühl [Typification]

KUMAR Anand, GHOSHAL Partha Pratim, BHARATI Kumar Avinash, Notes on the Type Specimens of Names in Aconitum (Ranunculaceae) at Central National Herbarium (CAL). Phytotaxa 475 (4): 209-238. 2020 [220] doi: 10.11646/phytotaxa.475.4.1 CHINA (Xizang*)

Aconitum ferox Wall. ex Ser. var. laxiflora Brühl [Typification]

KUMAR Anand, GHOSHAL Partha Pratim, BHARATI Kumar Avinash, Notes on the Type Specimens of Names in Aconitum (Ranunculaceae) at Central National Herbarium (CAL). Phytotaxa 475 (4): 209-238. 2020 [220] doi: 10.11646/phytotaxa.475.4.1 CHINA (Xizang); INDIA*

Aconitum gammiei Stapf [Typification]

KUMAR Anand, GHOSHAL Partha Pratim, BHARATI Kumar Avinash, Notes on the Type Specimens of Names in Aconitum (Ranunculaceae) at Central National Herbarium (CAL). Phytotaxa 475 (4): 209-238. 2020 [229] doi: 10.11646/phytotaxa.475.4.1 CHINA (Xizang); INDIA*

Aconitum haridasanii R. Tiwary, Harsh Singh \& D. Adhikari [New Species] IUCN: CE: B1b(i,ii,iii,v)c(i,ii,iii) +2b(i,ii,iii,v) c;C2a(i);D; Evidence: Morphology

TIWARY Raghuvar, SINGH Harsh, ADHIKARI Dibyendu, SINGH Prem Prakash, BARIK Saroj Kanta, Discovery of Aconitum haridasanii (Ranunculaceae), a New Species from Arunachal Pradesh, India. Phytotaxa 440 (3): 232-238. 2020 [232] doi: 10.11646/phytotaxa.440.3.5

CHINA (Xizang*)

Aconitum jucundum Diels [Typification]

KUMAR Anand, GHOSHAL Partha Pratim, BHARATI Kumar Avinash, Notes on the Type Specimens of Names in Aconitum (Ranunculaceae) at Central National Herbarium (CAL). Phytotaxa 475 (4): 209-238. 2020 [231] doi: 10.11646/phytotaxa.475.4.1 CHINA (Yunnan*)

Aconitum laxifoliatum W. T. Wang 疏叶乌头 [New Species] IUCN: NE; Evidence: Morphology

WANG Wen-tsai, Two New Species of Aconitum (Ranunculaceae) from Sichuan. Bulletin of Botanical Research 40 (1): 1-4. 2020 [4] doi: 10.7525/j.issn.1673-5102.2020.01.001

CHINA (Sichuan*)

Aconitum soongaricum Stapf [Typification]

KUMAR Anand, GHOSHAL Partha Pratim, BHARATI Kumar Avinash, Notes on the Type Specimens of Names in Aconitum (Ranunculaceae) at Central National Herbarium (CAL). Phytotaxa 475 (4): 209-238. 2020 [231] doi: 10.11646/phytotaxa.475.4.1 CHINA (Xinjiang); INDIA

Aconitum tenuigaleatum W. T. Wang 细盔乌头 [New Species] IUCN: NE; Evidence: Morphology

WANG Wen-tsai, Two New Species of Aconitum (Ranunculaceae) from Sichuan. Bulletin of Botanical Research 40 (1): 1-4. 2020 [1] doi: 10.7525/j.issn.1673-5102.2020.01.001

CHINA (Sichuan*)

Aconitum transsectum Diels [Typification]

KUMAR Anand, GHOSHAL Partha Pratim, BHARATI Kumar Avinash, Notes on the Type Specimens of Names in Aconitum (Ranunculaceae) at Central National Herbarium (CAL). Phytotaxa 475 (4): 209-238. 2020 [231] doi: 10.11646/phytotaxa.475.4.1 CHINA (Yunnan*)

Clematis connata DC. var. trullifera (Franch.) W. T. Wang [New Synonyms]

S.: Clematis dongchuanensis W. T. Wang 
杜诚, 刘军, 叶文, 廖帅, 葛斌杰, 刘冰, 马金双 (2021) 中国植物新分类群、新名称 2020 年度报告. 生物多样性, 29, 10111020. http://www.biodiversity-science.net/CN/10.17520/biods.2021122

YUAN Qiong, YANG Qin-er, The identity of Clematis dongchuanensis (Ranunculaceae) from Northeastern Yunnan, China. Phytotaxa 430 (1): 9-16. 2020 [10] doi: 10.11646/phytotaxa.430.1.2

CHINA (Guizhou, Yunnan*)

Clematis hastata Finet \& Gagnep. [New Synonyms]

S.: Clematis glabrifolia K. Sun \& M. S. Yan; Clematis hastata Finet \& Gagnep. var. micrantha W. T. Wang

YUAN Qiong, YANG Qin-er, Two New Synonyms of Clematis hastata (Ranunculaceae), a Species from the Daba Mountains Region in Central China. Phytotaxa 474 (2): 163-171. 2020 [164] doi: 10.11646/phytotaxa.474.2.6

CHINA (Chongqing*, Gansu, Shaanxi)

Clematis tientaiensis (M. Y. Fang) W. T. Wang 天台铁线莲 [New Combination]

B.: Clematis patens C.Morren \& Decne. subsp. tientaiensis M. Y. Fang

ZHANG Zhong-zhao, CHEN Zheng-hai, CHEN Feng, XIE Wen-yuan, LI Gen-you, Additional Notes on the Seed Plant Flora of Zhejiang( VII ). Journal of Zhejiang Forestry Science and Technology 40 (6): 52-55. 2020 [54] doi: 10.3969/j.issn.10013776.2020.06.008

CHINA (Zhejiang*)

Delphinium adenopodum W. T. Wang \& Z. Z. Yang 腺梗翠雀花 [New Species] IUCN: NE; Evidence: Morphology

WANG Wen-tsai, YANG Zong-zong, Two New Species of Delphinium from Xinjiang. Bulletin of Botanical Research 40 (6): $801-804$. 2020 [801] doi: 10.7525/j.issn.1673-5102.2020.06.001

CHINA (Xinjiang*)

Delphinium gongliuense W. T. Wang \& Z. Z. Yang 巩留翠雀花 [New Species] IUCN: NE; Evidence: Morphology

WANG Wen-tsai, YANG Zong-zong, Two New Species of Delphinium from Xinjiang. Bulletin of Botanical Research 40 (6): $801-804$. 2020 [804] doi: 10.7525/j.issn.1673-5102.2020.06.001

CHINA (Xinjiang*)

Halerpestes tricuspis (Maxim.) Hand.-Mazz. var. variifolia (Tamura) W. T. Wang [New Synonyms]

S.: Ranunculus kangmaensis W. T. Wang

ZHANG Meng, ZENG You-pai, YANG Qin-er, Ranunculus kangmaensis (Ranunculaceae), a New Synonym of Halerpestes tricuspis var. variifolia. Phytotaxa 434 (1): 101-112. 2020 [107] doi: 10.11646/phytotaxa.434.1.7

CHINA (Gansu, Qinghai, Sichuan, Xizang, Yunnan); NEPAL*

Hegemone micrantha (C. Winkl. \& Komarov) Butkov [New Record]

ERST Andrey Sergeevich, PENDRY Colin Alistair, IKEDA Hiroshi, WANG Wei, Hegemone micrantha (Ranunculaceae) — a New Record from Nepal and China. The Journal of Japanese Botany 95 (5): 303-305. 2020 [304]

CHINA (Xizang); AFGHANISTAN, KAZAKHSTAN, KYRGYZSTAN, NEPAL, TAJIKISTAN, TURKMENISTAN, UZBEKISTAN

Thalictrum foliolosum DC. [New Synonyms]

S.: Thalictrum mairei H. Lév.

ZENG You-pai, YUAN Qiong, YANG Qin-er, Thalictrum mairei (Ranunculaceae) Is Synonymous with T. foliolosum, not T. leuconotum. Phytotaxa 452 (2): 137-154. 2020 [149] doi: 10.11646/phytotaxa.452.2.3

CHINA (Sichuan, Xizang, Yunnan); BHUTAN, INDIA, MYANMAR, NEPAL*, THAILAND

Thalictrum foliolosum DC. [Typification]

ZENG You-pai, YUAN Qiong, YANG Qin-er, Thalictrum mairei (Ranunculaceae) Is Synonymous with T. foliolosum, not T. leuconotum. Phytotaxa 452 (2): 137-154. 2020 [149] doi: 10.11646/phytotaxa.452.2.3

CHINA (Sichuan, Xizang, Yunnan); BHUTAN, INDIA, MYANMAR, NEPAL*, THAILAND

Thalictrum jiulongense W. T. Wang 九龙唐松草 [New Species] IUCN: NE; Evidence: Morphology

WANG Wen-tsai, Three New Species of Thalictrum (Ranunculaceae) from Sichuan Province. Bulletin of Botanical Research 40 (1): 5-9. 2020 [9] doi: 10.7525/j.issn.1673-5102.2020.01.002

CHINA (Sichuan*)

Thalictrum kangdingense W. T. Wang 康定唐松草 [New Species] IUCN: NE; Evidence: Morphology

WANG Wen-tsai, Three New Species of Thalictrum (Ranunculaceae) from Sichuan Province. Bulletin of Botanical Research 40 (1): 5-9. 2020 [5] doi: 10.7525/j.issn.1673-5102.2020.01.002

CHINA (Sichuan*)

Thalictrum neurocarpum Royle [New Synonyms]

S.: Thalictrum punduanum Wall. var. hirtellum W. T. Wang

ZENG You-pai, YUAN Qiong, YANG Qin-er, Thalictrum punduanum var. hirtellum (Ranunculaceae), Another New Synonym of T. neurocarpum. Phytotaxa 442 (2): 89-100. 2020 [94] doi: 10.11646/phytotaxa.442.2.4

CHINA (Xizang); INDIA*, NEPAL 
杜诚, 刘军, 叶文, 廖帅, 葛斌杰, 刘冰, 马金双 (2021) 中国植物新分类群、新名称 2020 年度报告. 生物多样性, 29, 10111020. http://www.biodiversity-science.net/CN/10.17520/biods.2021122

Thalictrum tenuicaule W. T. Wang 细茎唐松草 [New Species] IUCN: NE; Evidence: Morphology

WANG Wen-tsai, Three New Species of Thalictrum (Ranunculaceae) from Sichuan Province. Bulletin of Botanical Research 40 (1): 5-9. 2020 [7] doi: 10.7525/j.issn.1673-5102.2020.01.002

CHINA (Sichuan*)

Thalictrum trichopus Franch. [New Synonyms]

S.: Thalictrum semiscandens W. W. Sm.

ZENG You-pai, YUAN Qiong, YANG Qin-er, Thalictrum semiscandens (Ranunculaceae), Almost Totally Overlooked since Its Publication, Is Merged with T. trichopus. Phytotaxa 451 (4): 283-293. 2020 [284] doi: 10.11646/phytotaxa.451.4.3

CHINA (Sichuan, Yunnan*)

Thalictrum trichopus Franch. [Typification]

ZENG You-pai, YUAN Qiong, YANG Qin-er, Thalictrum semiscandens (Ranunculaceae), Almost Totally Overlooked since Its Publication, Is Merged with T. trichopus. Phytotaxa 451 (4): 283-293. 2020 [284] doi: 10.11646/phytotaxa.451.4.3

CHINA (Sichuan, Yunnan*)

\section{Resedaceae 木櫂草科}

Stixis villiflora J. Y. Shen, S. Landrein, W. G. Wang \& X. D. Ma 多毛斑果藤 [New Species] IUCN: CR: B1ab(i,v)+2ab (i,v),D; Evidence: Morphology

SHEN Jian-yong, LANDREIN Sven, WANG Wen-guang, MA Xing-da, SHI Ji-pu, Stixis villiflora, a New Species of Resedaceae from Yunnan, China. Taiwania 65 (1): 10-14. 2020 [10] doi: 10.6165/tai.2020.65.10

CHINA (Yunnan*)

\section{Rhamnaceae 鼠李科}

Rhamnella intermedia Z. Qiang Lu \& Y. Shuai Sun [New Species] IUCN: NE; Evidence: ITS; Morphology

LU Zhi-qiang, SUN Yong-shuai, Rhamnella intermedia (Rhamnaceae), a New Evergreen Species from Southwest Guangxi. PhytoKeys 159: 115-126. 2020 [119] doi: 10.3897/phytokeys.159.53177

CHINA (Guangxi*)

\section{Rosaceae 萻薇科}

Eriobotrya bengalensis (Roxb.) Kurz f. intermedia J. E. Vidal [Typification]

LIU Bin-bin, LIU Guang-ning, HONG De-yuan, WEN Jun, Typification of 23 Names in Eriobotrya (Maleae, Rosaceae). PhytoKeys 139: 99-118. 2020 [103] doi: 10.3897/phytokeys.139.47967

CHINA (Yunnan); MYANMAR*, VIET NAM

Eriobotrya bengalensis (Roxb.) Kurz var. angustifolia Cardot [Typification]

LIU Bin-bin, LIU Guang-ning, HONG De-yuan, WEN Jun, Typification of 23 Names in Eriobotrya (Maleae, Rosaceae). PhytoKeys 139: 99-118. 2020 [103] doi: 10.3897/phytokeys.139.47967

CHINA (Yunnan*)

Eriobotrya brackloi Hand.-Mazz. [Typification]

LIU Bin-bin, LIU Guang-ning, HONG De-yuan, WEN Jun, Typification of 23 Names in Eriobotrya (Maleae, Rosaceae). PhytoKeys 139: 99-118. 2020 [103] doi: 10.3897/phytokeys.139.47967

CHINA (Guangdong*)

Eriobotrya brackloi Hand.-Mazz. var. atrichophylla Hand.-Mazz. [Typification]

LIU Bin-bin, LIU Guang-ning, HONG De-yuan, WEN Jun, Typification of 23 Names in Eriobotrya (Maleae, Rosaceae). PhytoKeys 139: 99-118. 2020 [104] doi: 10.3897/phytokeys.139.47967

CHINA (Hunan*)

Eriobotrya grandiflora Rehder \& E. H. Wilson [Typification]

LIU Bin-bin, LIU Guang-ning, HONG De-yuan, WEN Jun, Typification of 23 Names in Eriobotrya (Maleae, Rosaceae). PhytoKeys 139: 99-118. 2020 [107] doi: 10.3897/phytokeys.139.47967

CHINA (Sichuan*)

Eriobotrya henryi Nakai [Typification]

LIU Bin-bin, LIU Guang-ning, HONG De-yuan, WEN Jun, Typification of 23 Names in Eriobotrya (Maleae, Rosaceae). PhytoKeys 139: 99-118. 2020 [107] doi: 10.3897/phytokeys.139.47967

CHINA (Yunnan*) 
杜诚, 刘军, 叶文, 廖帅, 葛斌杰, 刘冰, 马金双 (2021) 中国植物新分类群、新名称 2020 年度报告. 生物多样性, 29, 10111020. http://www.biodiversity-science.net/CN/10.17520/biods.2021122

Eriobotrya laoshanica W. B. Liao, Q. Fan \& Su F. Chen 老山枇杷 [New Species] IUCN: CR: B2ab(v); Evidence: Chloroplast WGS; Morphology

CHEN Su-fang, MENG Kai-kai, GUO Xi-bing, ZHAO Wan-yi, LIAO Wen-bo, FAN Qiang, A New Species of Eriobotrya (Rosaceae) from Yunnan Province, China. PhytoKeys 146: 61-69. 2020 [64] doi: 10.3897/phytokeys.146.50728

CHINA (Yunnan*)

Eriobotrya prinoides Rehder \& E. H. Wilson [Typification]

LIU Bin-bin, LIU Guang-ning, HONG De-yuan, WEN Jun, Typification of 23 Names in Eriobotrya (Maleae, Rosaceae). PhytoKeys 139: 99-118. 2020 [110] doi: 10.3897/phytokeys.139.47967

CHINA (Yunnan*)

Eriobotrya salwinensis Hand.-Mazz. [Typification]

LIU Bin-bin, LIU Guang-ning, HONG De-yuan, WEN Jun, Typification of 23 Names in Eriobotrya (Maleae, Rosaceae). PhytoKeys 139: 99-118. 2020 [111] doi: 10.3897/phytokeys.139.47967

CHINA (Yunnan*)

Eriobotrya serrata J. E. Vidal [Typification]

LIU Bin-bin, LIU Guang-ning, HONG De-yuan, WEN Jun, Typification of 23 Names in Eriobotrya (Maleae, Rosaceae). PhytoKeys 139: 99-118. 2020 [111] doi: 10.3897/phytokeys.139.47967

CHINA (Guangxi, Yunnan); LAOS*

Geum sunhangii D. G. Zhang, T. Deng, Z. Y. Lv \& Zhi M. Li 神农花 [New Species] IUCN: NT; Evidence: nrITS, trnL-trnF; Morphology

LV Zhen-yu, ZHANG Dai-gui, HUANG Xian-han, WANG Heng-chang, YANG Jing-yuan, TOJIBAEV Komiljon, DENG Tao, LI Zhimin, Geum sunhangii (Rosaceae), a New Species from Hubei Province, China. PhytoKeys 156: 113-124. 2020 [115] doi: 10.3897/phytokeys.156.37277

CHINA (Hubei*)

Hiptage cavaleriei H. Lév. [Typification]

LIU Bin-bin, LIU Guang-ning, HONG De-yuan, WEN Jun, Typification of 23 Names in Eriobotrya (Maleae, Rosaceae). PhytoKeys 139: 99-118. 2020 [112] doi: 10.3897/phytokeys.139.47967

CHINA (Guizhou*)

Hiptage lushuiensis S. P. Dong, K. Tan \& M. X. Ren 泸水风笔果 [New Species] IUCN: CR; Evidence: Morphology

DONG Shu-peng, TAN Ke, REN Ming-xun, A New Species of Hiptage (Malpighiaceae) from Nujiang Gorge, Southwest China. Nordic Journal of Botany 38 (e02436): 1-6. 2020 [2] doi: 10.1111/njb.02436

CHINA (Yunnan*)

Pourthiaea amphidoxa (C. K. Schneid.) Stapf [Typification]

GUO Wei, FAN Qiang, ZHANG Xian-zhi, LIAO Wen-bo, WANG Long-yuan, WU Wei, POTTER Daniel, Molecular Reappraisal of Relationships between Photinia, Stranvaesia and Heteromeles (Rosaceae, Maleae). Phytotaxa 447 (2): 103-115. 2020 [111] doi: 10.11646/phytotaxa.447.2.3

CHINA (Sichuan*)

Pourthiaea amphidoxa (C. K. Schneid.) Stapf var. amphileia (Hand.-Mazz.) W. Guo, W. B. Liao \& Long Y. Wang [New Combination] B.: Photinia amphidoxa Rehder \& E. H. Wilson var. amphileia Hand.-Mazz.

GUO Wei, FAN Qiang, ZHANG Xian-zhi, LIAO Wen-bo, WANG Long-yuan, WU Wei, POTTER Daniel, Molecular Reappraisal of Relationships between Photinia, Stranvaesia and Heteromeles (Rosaceae, Maleae). Phytotaxa 447 (2): 103-115. 2020 [111] doi: 10.11646/phytotaxa.447.2.3

CHINA (Hunan*)

Pourthiaea amphidoxa (C. K. Schneid.) Stapf var. amphileia (Hand.-Mazz.) W. Guo, W. B. Liao \& Long Y. Wang [Typification] GUO Wei, FAN Qiang, ZHANG Xian-zhi, LIAO Wen-bo, WANG Long-yuan, WU Wei, POTTER Daniel, Molecular Reappraisal of Relationships between Photinia, Stranvaesia and Heteromeles (Rosaceae, Maleae). Phytotaxa 447 (2): 103-115. 2020 [111] doi: 10.11646/phytotaxa.447.2.3

CHINA (Hunan*)

Pourthiaea tomentosa (T. T. Yu \& T. C. Ku) Long Y. Wang, W. Guo \& W. B. Liao [New Combination]

B.: Stranvaesia tomentosa T. T. Yu \& T. C. Ku

GUO Wei, FAN Qiang, ZHANG Xian-zhi, LIAO Wen-bo, WANG Long-yuan, WU Wei, POTTER Daniel, Molecular Reappraisal of Relationships between Photinia, Stranvaesia and Heteromeles (Rosaceae, Maleae). Phytotaxa 447 (2): 103-115. 2020 [111] doi: 10.11646/phytotaxa.447.2.3

CHINA (Chongqing*) 
杜诚, 刘军, 叶文, 廖帅, 葛斌杰, 刘冰, 马金双 (2021) 中国植物新分类群、新名称 2020 年度报告. 生物多样性, 29, 10111020. http://www.biodiversity-science.net/CN/10.17520/biods.2021122

Pourthiaea tomentosa (T. T. Yu \& T. C. Ku) Long Y. Wang, W. Guo \& W. B. Liao [Typification]

GUO Wei, FAN Qiang, ZHANG Xian-zhi, LIAO Wen-bo, WANG Long-yuan, WU Wei, POTTER Daniel, Molecular Reappraisal of Relationships between Photinia, Stranvaesia and Heteromeles (Rosaceae, Maleae). Phytotaxa 447 (2): 103-115. 2020 [111] doi: 10.11646/phytotaxa.447.2.3

CHINA (Chongqing*)

Rhaphiolepis Lindl. [New Synonyms]

S.: Eriobotrya Lindl.

LIU Bin-bin, LIU Guang-ning, HONG De-yuan, WEN Jun, Eriobotrya Belongs to Rhaphiolepis (Maleae, Rosaceae): Evidence from Chloroplast Genome and Nuclear Ribosomal DNA Data. Frontiers in Plant Science 10 (1731): 1-14. 2020 [9] doi: 10.3389/fpls.2019.01731

CHINA

Rhaphiolepis $\times$ daduheensis (Hui Z. Zhang ex W. B. Liao, Q. Fan \& M. Y. Ding) B. B. Liu \& J. Wen [New Combination]

B.: Eriobotrya $\times$ daduheensis Hui Z. Zhang ex W. B. Liao, Q. Fan \& M. Y. Ding

LIU Bin-bin, LIU Guang-ning, HONG De-yuan, WEN Jun, Eriobotrya Belongs to Rhaphiolepis (Maleae, Rosaceae): Evidence from Chloroplast Genome and Nuclear Ribosomal DNA Data. Frontiers in Plant Science 10 (1731): 1-14. 2020 [10] doi: 10.3389/fpls.2019.01731

CHINA (Sichuan)

Rhaphiolepis bengalensis (Roxb.) B. B. Liu \& J. Wen f. angustifolia (Cardot) B. B. Liu \& J. Wen 窄叶南亚枇杷 [New Combination] B.: Eriobotrya bengalensis (Roxb.) Hook. f. var. angustifolia Cardot

LIU Bin-bin, WANG Yu-bing, HONG De-yuan, WEN Jun, A Synopsis of the Expanded Rhaphiolepis (Maleae, Rosaceae). PhytoKeys 154: 19-55. 2020 [23] doi: 10.3897/phytokeys.154.52790

CHINA (Yunnan*)

Rhaphiolepis cavaleriei (H. Lév.) B. B. Liu \& J. Wen [New Combination]

B.: Hiptage cavaleriei H. Lév.

LIU Bin-bin, LIU Guang-ning, HONG De-yuan, WEN Jun, Eriobotrya Belongs to Rhaphiolepis (Maleae, Rosaceae): Evidence from Chloroplast Genome and Nuclear Ribosomal DNA Data. Frontiers in Plant Science 10 (1731): 1-14. 2020 [10] doi: 10.3389/fpls.2019.01731

CHINA (Fujian, Guangdong, Guangxi, Guizhou, Hubei, Hunan, Jiangxi, Sichuan); VIET NAM

Rhaphiolepis deflexa (Hemsl.) B. B. Liu \& J. Wen [New Combination]

B.: Photinia deflexa Hemsl.

LIU Bin-bin, LIU Guang-ning, HONG De-yuan, WEN Jun, Eriobotrya Belongs to Rhaphiolepis (Maleae, Rosaceae): Evidence from Chloroplast Genome and Nuclear Ribosomal DNA Data. Frontiers in Plant Science 10 (1731): 1-14. 2020 [10] doi: 10.3389/fpls.2019.01731

CHINA (Guangdong, Hainan, Taiwan); VIET NAM

Rhaphiolepis elliptica (Lindl.) B. B. Liu \& J. Wen [New Combination]

B.: Eriobotrya elliptica Lindl.

LIU Bin-bin, LIU Guang-ning, HONG De-yuan, WEN Jun, Eriobotrya Belongs to Rhaphiolepis (Maleae, Rosaceae): Evidence from Chloroplast Genome and Nuclear Ribosomal DNA Data. Frontiers in Plant Science 10 (1731): 1-14. 2020 [10] doi: 10.3389/fpls.2019.01731

CHINA (Xizang); NEPAL

Rhaphiolepis ferruginea F. P. Metcalf var. serrata F. P. Metcalf 齿叶锈毛石斑木 [Typification]

LIU Bin-bin, WANG Yu-bing, HONG De-yuan, WEN Jun, A Synopsis of the Expanded Rhaphiolepis (Maleae, Rosaceae). PhytoKeys 154: 19-55. 2020 [31] doi: 10.3897/phytokeys.154.52790

CHINA (Fujian, Guangdong*, Guangxi)

Rhaphiolepis fulvicoma (Chun ex W. B. Liao, F. F. Li \& D. F. Cui) B. B. Liu \& J. Wen [New Combination]

B.: Eriobotrya fulvicoma Chun ex W. B. Liao, F. F. Li \& D. F. Cui

LIU Bin-bin, LIU Guang-ning, HONG De-yuan, WEN Jun, Eriobotrya Belongs to Rhaphiolepis (Maleae, Rosaceae): Evidence from Chloroplast Genome and Nuclear Ribosomal DNA Data. Frontiers in Plant Science 10 (1731): 1-14. 2020 [10] doi: 10.3389/fpls.2019.01731

CHINA (Guangdong)

Rhaphiolepis gracilis Nakai [Typification]

LIU Bin-bin, WANG Yu-bing, HONG De-yuan, WEN Jun, A Synopsis of the Expanded Rhaphiolepis (Maleae, Rosaceae). PhytoKeys 154: 19-55. 2020 [34] doi: 10.3897/phytokeys.154.52790

CHINA (Zhejiang*)

Rhaphiolepis hainanensis F. P. Metcalf [Typification] 
杜诚, 刘军, 叶文, 廖帅, 葛斌杰, 刘冰, 马金双 (2021) 中国植物新分类群、新名称 2020 年度报告. 生物多样性, 29, 10111020. http://www.biodiversity-science.net/CN/10.17520/biods.2021122

LIU Bin-bin, WANG Yu-bing, HONG De-yuan, WEN Jun, A Synopsis of the Expanded Rhaphiolepis (Maleae, Rosaceae). PhytoKeys 154: 19-55. 2020 [38] doi: 10.3897/phytokeys.154.52790

CHINA (Hainan*)

Rhaphiolepis henryi (Nakai) B. B. Liu \& J. Wen [New Combination]

B.: Eriobotrya henryi Nakai

LIU Bin-bin, LIU Guang-ning, HONG De-yuan, WEN Jun, Eriobotrya Belongs to Rhaphiolepis (Maleae, Rosaceae): Evidence from Chloroplast Genome and Nuclear Ribosomal DNA Data. Frontiers in Plant Science 10 (1731): 1-14. 2020 [11] doi: 10.3389/fpls.2019.01731

CHINA (Guizhou, Yunnan); MYANMAR

Rhaphiolepis loquata B. B. Liu \& J. Wen [New Name]

R.: Mespilus japonica Thunb.

LIU Bin-bin, LIU Guang-ning, HONG De-yuan, WEN Jun, Eriobotrya Belongs to Rhaphiolepis (Maleae, Rosaceae): Evidence from Chloroplast Genome and Nuclear Ribosomal DNA Data. Frontiers in Plant Science 10 (1731): 1-14. 2020 [11] doi: 10.3389/fpls.2019.01731

CHINA (Chongqing, Hubei); INDIA, JAPAN, KOREA

Rhaphiolepis malipoensis (K. C. Kuan) B. B. Liu \& J. Wen [New Combination]

B.: Eriobotrya malipoensis K. C. Kuan

LIU Bin-bin, LIU Guang-ning, HONG De-yuan, WEN Jun, Eriobotrya Belongs to Rhaphiolepis (Maleae, Rosaceae): Evidence from Chloroplast Genome and Nuclear Ribosomal DNA Data. Frontiers in Plant Science 10 (1731): 1-14. 2020 [11] doi: 10.3389/fpls.2019.01731

CHINA (Yunnan)

Rhaphiolepis obovata (W. W. Sm.) B. B. Liu \& J. Wen [New Combination]

B.: Eriobotrya obovata W. W. Sm.

LIU Bin-bin, LIU Guang-ning, HONG De-yuan, WEN Jun, Eriobotrya Belongs to Rhaphiolepis (Maleae, Rosaceae): Evidence from Chloroplast Genome and Nuclear Ribosomal DNA Data. Frontiers in Plant Science 10 (1731): 1-14. 2020 [11] doi: 10.3389/fpls.2019.01731

CHINA (Yunnan)

Rhaphiolepis parvibracteolata Merr. [Typification]

LIU Bin-bin, WANG Yu-bing, HONG De-yuan, WEN Jun, A Synopsis of the Expanded Rhaphiolepis (Maleae, Rosaceae). PhytoKeys 154: 19-55. 2020 [35] doi: 10.3897/phytokeys.154.52790

CHINA (Hainan*)

Rhaphiolepis prinoides (Rehder \& E. H. Wilson) B. B. Liu \& J. Wen [New Combination]

B.: Eriobotrya prinoides Rehder \& E. H. Wilson

LIU Bin-bin, LIU Guang-ning, HONG De-yuan, WEN Jun, Eriobotrya Belongs to Rhaphiolepis (Maleae, Rosaceae): Evidence from Chloroplast Genome and Nuclear Ribosomal DNA Data. Frontiers in Plant Science 10 (1731): 1-14. 2020 [11] doi: 10.3389/fpls.2019.01731

CHINA (Sichuan, Yunnan); LAOS

Rhaphiolepis salwinensis (Hand.-Mazz.) B. B. Liu \& J. Wen [New Combination]

B.: Eriobotrya salwinensis Hand.-Mazz.

LIU Bin-bin, LIU Guang-ning, HONG De-yuan, WEN Jun, Eriobotrya Belongs to Rhaphiolepis (Maleae, Rosaceae): Evidence from Chloroplast Genome and Nuclear Ribosomal DNA Data. Frontiers in Plant Science 10 (1731): 1-14. 2020 [11] doi: 10.3389/fpls.2019.01731

CHINA (Yunnan, Xizang); INDIA, MYANMAR

Rhaphiolepis seguinii (H. Lév.) B. B. Liu \& J. Wen [New Combination]

B.: Symplocos seguinii H. Lév.

LIU Bin-bin, LIU Guang-ning, HONG De-yuan, WEN Jun, Eriobotrya Belongs to Rhaphiolepis (Maleae, Rosaceae): Evidence from Chloroplast Genome and Nuclear Ribosomal DNA Data. Frontiers in Plant Science 10 (1731): 1-14. 2020 [11] doi: 10.3389/fpls.2019.01731

CHINA (Guizhou, Yunnan)

Rhaphiolepis serrata (J. E. Vidal) B. B. Liu \& J. Wen [New Combination]

B.: Eriobotrya serrata J. E. Vidal

LIU Bin-bin, LIU Guang-ning, HONG De-yuan, WEN Jun, Eriobotrya Belongs to Rhaphiolepis (Maleae, Rosaceae): Evidence from Chloroplast Genome and Nuclear Ribosomal DNA Data. Frontiers in Plant Science 10 (1731): 1-14. 2020 [12] doi: 10.3389/fpls.2019.01731

CHINA (Guangxi, Yunnan); LAOS 
杜诚, 刘军, 叶文, 廖帅, 葛斌杰, 刘冰, 马金双 (2021) 中国植物新分类群、新名称 2020 年度报告. 生物多样性, 29, 10111020. http://www.biodiversity-science.net/CN/10.17520/biods.2021122

Rhaphiolepis tengyuehensis (W. W. Sm.) B. B. Liu \& J. Wen [New Combination]

B.: Eriobotrya tengyuehensis W. W. Sm.

LIU Bin-bin, LIU Guang-ning, HONG De-yuan, WEN Jun, Eriobotrya Belongs to Rhaphiolepis (Maleae, Rosaceae): Evidence from Chloroplast Genome and Nuclear Ribosomal DNA Data. Frontiers in Plant Science 10 (1731): 1-14. 2020 [12] doi: 10.3389/fpls.2019.01731

CHINA (Xizang, Yunnan); MYANMAR

Rhaphiolepis umbellata (Thunb.) Makino var. liukiuensis Koidz. 琉球厚叶石斑木 [Typification]

LIU Bin-bin, WANG Yu-bing, HONG De-yuan, WEN Jun, A Synopsis of the Expanded Rhaphiolepis (Maleae, Rosaceae). PhytoKeys 154: 19-55. 2020 [48] doi: 10.3897/phytokeys.154.52790

CHINA (Taiwan); JAPAN, KOREA*

Rhaphiolepis williamtelliana (M. F. Fay \& Christenh.) B. B. Liu \& J. Wen [New Combination]

B.: Pyrus williamtelliana M. F. Fay \& Christenh.

LIU Bin-bin, LIU Guang-ning, HONG De-yuan, WEN Jun, Eriobotrya Belongs to Rhaphiolepis (Maleae, Rosaceae): Evidence from Chloroplast Genome and Nuclear Ribosomal DNA Data. Frontiers in Plant Science 10 (1731): 1-14. 2020 [12] doi: 10.3389/fpls.2019.01731

CHINA (Guangdong, Guangxi, Hainan, Hongkong, Xizang); VIET NAM

Rubus jingningensis Z. H. Chen, F. Chen \& F. G. Zhang 景宁悬钩子 [New Species]

ZHAO Chang-gao, XU Hong-feng, ZHANG Fang-gang, CHEN Feng, XIE Wen-yuan, CHEN Zheng-hai, Rubus jingningensis (Rosaceae), a New Species from Zhejiang. Journal of Hangzhou Normal University (Natural Science Edition) 19 (3): $244-246$ (316). 2020 [244] doi: 10.12191/j.issn.1674-232X.2020.03.004

CHINA (Zhejiang*)

Rubus parvifolius L. f. alba K. Ye 白花茅莓 [New Form]

YE Kang, ZHU Xin-xin, New Records and New Form of Spermatophyte in Anhui Province. Journal of Plant Resources and Environment 29 (6): 72-74. 2020 [73] doi: 10.3969/j.issn.1674-7895.2020.06.10

CHINA (Anhui*)

Rubus tephrodes Hance 灰白毛莓 [New Synomyms]

S.: Rubus tephrodes Hance var. ampliforus (H. Lév. \& Vaniot) Hand.-Mazz.

XU Hong-feng, CHEN Lin, ZHANG Fang-gang, MEI Xu-dong, XIE Wen-yuan, CHEN Zheng-hai, New Materials of Rubus L. in Zhejiang (II). Journal of Zhejiang Forestry Science and Technology 40 (3): 85-90. 2020 [87] doi: 10.3969/j.issn.10013776.2020.04.015

CHINA (Guangdong*, Zhejiang)

Sorbus gongshanensis X. F. Gao \& Meng Li [New Species] IUCN: DD; Evidence: Morphology

LI Meng, GAO Xin-fen, TIAN Jing, JU Wen-bin, Sorbus gongshanensis (Rosaceae), a New Species from the Hengduan Mountains, China. PhytoKeys 144: 1-9. 2020 [2] doi: 10.3897/phytokeys.144.48516

CHINA (Xizang, Yunnan*)

Stranvaesia bodinieri (H. Lév.) Long Y. Wang, W. B. Liao \& W. Guo [New Combination]

B.: Photinia bodinieri H. Lév.

GUO Wei, FAN Qiang, ZHANG Xian-zhi, LIAO Wen-bo, WANG Long-yuan, WU Wei, POTTER Daniel, Molecular Reappraisal of Relationships between Photinia, Stranvaesia and Heteromeles (Rosaceae, Maleae). Phytotaxa 447 (2): 103-115. 2020 [110] doi: 10.11646/phytotaxa.447.2.3

CHINA (Guizhou*)

Stranvaesia bodinieri (H. Lév.) Long Y. Wang, W. B. Liao \& W. Guo [Typification]

GUO Wei, FAN Qiang, ZHANG Xian-zhi, LIAO Wen-bo, WANG Long-yuan, WU Wei, POTTER Daniel, Molecular Reappraisal of Relationships between Photinia, Stranvaesia and Heteromeles (Rosaceae, Maleae). Phytotaxa 447 (2): 103-115. 2020 [110] doi: 10.11646/phytotaxa.447.2.3

CHINA (Guizhou*)

Stranvaesia nussia (Buch.-Ham. ex D. Don) Decne. [Typification]

GUO Wei, FAN Qiang, ZHANG Xian-zhi, LIAO Wen-bo, WANG Long-yuan, WU Wei, POTTER Daniel, Molecular Reappraisal of Relationships between Photinia, Stranvaesia and Heteromeles (Rosaceae, Maleae). Phytotaxa 447 (2): 103-115. 2020 [110] doi: 10.11646/phytotaxa.447.2.3

CHINA (Xizang, Yunnan); INDIA, LAOS, MYANMAR, NEPAL*, PHILIPPINES, THAILAND

Stranvaesia oblanceolata (Rehder \& E. H. Wilson) Stapf [Typification] 
杜诚, 刘军, 叶文, 廖帅, 葛斌杰, 刘冰, 马金双 (2021) 中国植物新分类群、新名称 2020 年度报告. 生物多样性, 29, 10111020. http://www.biodiversity-science.net/CN/10.17520/biods.2021122

GUO Wei, FAN Qiang, ZHANG Xian-zhi, LIAO Wen-bo, WANG Long-yuan, WU Wei, POTTER Daniel, Molecular Reappraisal of Relationships between Photinia, Stranvaesia and Heteromeles (Rosaceae, Maleae). Phytotaxa 447 (2): 103-115. 2020 [110] doi: 10.11646/phytotaxa.447.2.3

CHINA (Yunnan*)

Symplocos arisanensis Hayata 阿里山山矾 [Correction]

A.: Symplocos lancifolia auct. non Siebold \& Zucc.: C. Z. Zheng in Y. Y. Fang, Fl. Zhejiang 5: 81, 1992; Y. F. Wu in Y. F. Wu \& S. M. Hwang, Fl. Reipubl. Popularis Sin. 60(2): 51, 1987, p.p.; Y. F. Wu \& H. P. Nooteboom in C. Y. Wu \& al., Fl. China 15: 24

CHEN Zheng-hai, CHEN Xian-xing, LI Gen-you, XIE Wen-yuan, CHEN Feng, Taxonomic Revision of the Genus Symplocos Jacq. from Zhejiang Province. Journal of Wenzhou University (Natural Science Edition) 41 (1): 47-55. 2020 [48] doi: 10.3875/j.issn.16743563.2020.01.007

CHINA (Taiwan, Zhejiang); JAPAN, KOREA

Symplocos botryantha Franch. 总状山矾 [Correction]

A.: Symplocos sumuntia auct. non Buch.-Ham. ex D. Don: Y. F. Wu \& H. P. Nooteboom in C. Y. Wu \& al., Fl. China 15: $243,1996$.

CHEN Zheng-hai, CHEN Xian-xing, LI Gen-you, XIE Wen-yuan, CHEN Feng, Taxonomic Revision of the Genus Symplocos Jacq. from Zhejiang Province. Journal of Wenzhou University (Natural Science Edition) 41 (1): 47-55. 2020 [49] doi: 10.3875/j.issn.16743563.2020.01.007

CHINA (Zhejiang); JAPAN, KOREA

Symplocos botryantha Franch. 总状山矾 [Correction]

A.: Symplocos sumuntia auct. non Buch.-Ham. ex D. Don: Y. F. Wu \& H. P. Nooteboom in C. Y. Wu \& al., Fl. China 15: 243 , 1996.

CHEN Zheng-hai, CHEN Xian-xing, LI Gen-you, XIE Wen-yuan, CHEN Feng, Taxonomic Revision of the Genus Symplocos Jacq. from Zhejiang Province. Journal of Wenzhou University (Natural Science Edition) 41 (1): 47-55. 2020 [49] doi: 10.3875/j.issn.16743563.2020.01.007

CHINA (Zhejiang); JAPAN, KOREA

Symplocos caudata Wall. ex G. Don 山矾 [Correction]

A.: Symplocos sumuntia auct. non Buch.-Ham. ex D. Don: Y. F. Wu in Y. F. Wu \& S. M. Hwang, Fl. Reipubl. Popularis Sin. 60(2): 22: 11-13, 1987; C. Z. Zheng in Y. Y. Fang, Fl. Zhejiang ed. 2, 5: 84, 1992; Y. F. Wu \& H. P. Nooteboom in C. Y. Wu \& al., Fl. C

CHEN Zheng-hai, CHEN Xian-xing, LI Gen-you, XIE Wen-yuan, CHEN Feng, Taxonomic Revision of the Genus Symplocos Jacq. from Zhejiang Province. Journal of Wenzhou University (Natural Science Edition) 41 (1): 47-55. 2020 [49] doi: 10.3875/j.issn.16743563.2020.01.007

CHINA (Zhejiang)

Symplocos coreana (H. Lév.) Ohwi 朝鲜白檀 [New Record]

CHEN Zheng-hai, CHEN Xian-xing, LI Gen-you, XIE Wen-yuan, CHEN Feng, Taxonomic Revision of the Genus Symplocos Jacq. from Zhejiang Province. Journal of Wenzhou University (Natural Science Edition) 41 (1): 47-55. 2020 [52] doi: 10.3875/j.issn.16743563.2020.01.007

CHINA (Zhejiang); JAPAN, KOREA

Symplocos phyllocalyx C. B. Clarke 叶蕶山矾 [Correction]

A.: Smplocos lucida auct. non (Thunb.) Siebold \& Zucc.: Y. F. Wu \& H. P. Nooteboom in C. Y. Wu \& al., Fl. China 15: 242 , 1996.

CHEN Zheng-hai, CHEN Xian-xing, LI Gen-you, XIE Wen-yuan, CHEN Feng, Taxonomic Revision of the Genus Symplocos Jacq. from Zhejiang Province. Journal of Wenzhou University (Natural Science Edition) 41 (1): 47-55. 2020 [50] doi: 10.3875/j.issn.16743563.2020.01.007

CHINA (Zhejiang)

Symplocos prunifolia Siebold et Zucc. 黑山山矾 [New Synomyms]

S.: Symplocos heishanensis Hayata

CHEN Zheng-hai, CHEN Xian-xing, LI Gen-you, XIE Wen-yuan, CHEN Feng, Taxonomic Revision of the Genus Symplocos Jacq. from Zhejiang Province. Journal of Wenzhou University (Natural Science Edition) 41 (1): 47-55. 2020 [51] doi: 10.3875/j.issn.16743563.2020.01.007

CHINA (Zhejiang); JAPAN

Symplocos seguinii H. Lév. [Typification]

LIU Bin-bin, LIU Guang-ning, HONG De-yuan, WEN Jun, Typification of 23 Names in Eriobotrya (Maleae, Rosaceae). PhytoKeys 139: 99-118. 2020 [112] doi: 10.3897/phytokeys.139.47967

CHINA (Guizhou*)

Symplocos tanakana Nakai 白檀 [Correction]

A.: Symplocos paniculata auct. non (Thunb. ex Murray) Miq.: Icon. Cormophyt. Sin. 3: 331, 1974; Fl. Jiangsu: 615, 1977 ; Medic. Pl. Zhejiang: 992, 1980; T. L. Wu, Fl. Guangdong 1: 422-423, 1987; Fl. Reipubl. Popularis Sin. 60(2): 72, 1987; Fl. China 15: 2 
杜诚, 刘军, 叶文, 廖帅, 葛斌杰, 刘冰, 马金双 (2021) 中国植物新分类群、新名称 2020 年度报告. 生物多样性, 29, 10111020. http://www.biodiversity-science.net/CN/10.17520/biods.2021122

CHEN Zheng-hai, CHEN Xian-xing, LI Gen-you, XIE Wen-yuan, CHEN Feng, Taxonomic Revision of the Genus Symplocos Jacq. from Zhejiang Province. Journal of Wenzhou University (Natural Science Edition) 41 (1): 47-55. 2020 [48] doi: 10.3875/j.issn.16743563.2020.01.007

CHINA (Guangdong, Zhejiang); JAPAN, KOREA

Symplocos theophrastifolia Siebold \& Zucc. 黄牛奶树 [Correction]

A.: Symplocos laurina auct. non (Retz.) Wall.: Y. F. Wu in Y. F. Wu \& S. M. Hwang, Fl. Reipubl. Popularis Sin. 60(2): 55, 1987; C. Z. Zheng in Y. Y. Fang, Fl. Zhejiang ed. 2, 5: 81, 1992

CHEN Zheng-hai, CHEN Xian-xing, LI Gen-you, XIE Wen-yuan, CHEN Feng, Taxonomic Revision of the Genus Symplocos Jacq. from Zhejiang Province. Journal of Wenzhou University (Natural Science Edition) 41 (1): 47-55. 2020 [51] doi: 10.3875/j.issn.16743563.2020.01.007

CHINA (Zhejiang)

\section{Rubiaceae 茜草科}

Dimetia brevipetiolata R. J. Wang 短柄藤耳草 [New Species] IUCN: LC; Evidence: ITS, petD; Morphology

ZHANG Ying, JIANG Guo-bin, WANG Rui-jiang, Dimetia brevipetiolata (Spermacoceae: Rubiaceae): a New Species from Guangxi, China. Phytotaxa 428 (1): 43-50. 2020 [45] doi: 10.11646/phytotaxa.428.1.4

CHINA (Guangxi*)

Hedyotis zhihaoana Huan C. Wang \& Xiao Lan Liu 志浩耳草 [New Species] IUCN: NE; Evidence: ITS; Morphology

LIU Xiao-lan, LI Yu-ran, YANG Feng, WANG Huan-chong, Hedyotis zhihaoana (Rubiaceae), a New Species from Yunnan, Southwest China. Phytotaxa 461 (3): 166-174. 2020 [168] doi: 10.11646/phytotaxa.461.3.3

CHINA (Yunnan*)

Involucrella lithophila M. D. Yuan \& R. J. Wang [New Species] IUCN: LC; Evidence: ITS, petD; Morphology

YUAN Ming-deng, WANG Rui-jiang, Involucrella lithophila (Rubiaceae: Spermacoceae): a New Species from Guangxi, China. Phytotaxa 464 (1): 59-68. 2020 [60] doi: 10.11646/phytotaxa.464.1.4

CHINA (Guangxi*)

\section{Rutaceae 芸香科}

Murraya macrophylla (C. C. Huang) F. J. Mou \& D. X. Zhang 大叶九里香 [New Combination]

B.: Murraya kwangsiensis (C. C. Huang) C. C. Huang var. macrophylla C. C. Huang

MOU Feng-juan, HU Xiu, MA Shi-zhu, LI Yi-guo, Murraya macrophylla, A Name at New Rank and New Combination of Rutaceae and Its Supplements of Biological Characteristics. Guihaia 40 (2): 272-276. 2020 [274] doi: 10.11931/guihaia.gxzw201811001

CHINA (Guangxi*)

Zanthoxylum huangianum Z. H. Chen \& F. Chen 梗花椒 [New Name]

R.: Zanthoxylum stipitatum C. C. Huang

CHEN Feng, XIE Wen-yuan, ZHANG Shui-li, ZHANG Fen-yao, CHEN Zheng-hai, Additional Notes on the Seed Plants in Zhejiang (II). Journal of Hangzhou Normal University (Natural Science Edition) 19 (6): 96-102. 2020 [98] doi: 10.12191/j.issn.1674232X.2020.06.014

CHINA (Fujian, Guangdong*, Guangxi, Hunan, Zhejiang)

Zanthoxylum molle Rehder 朵椒 [Correction]

A.: Zanthoxylum ailanthoides Siebold \& Zucc. var. pubescens auct. non. HatuS.: Z. Wei \& Y. B. Zhang in Z. Wei \& Y. Q. He, Fl. Zhejiang 3: 424. 1993

CHEN Feng, XIE Wen-yuan, ZHANG Shui-li, ZHANG Fen-yao, CHEN Zheng-hai, Additional Notes on the Seed Plants in Zhejiang (II). Journal of Hangzhou Normal University (Natural Science Edition) 19 (6): 96-102. 2020 [97] doi: 10.12191/j.issn.1674232X.2020.06.014

CHINA (Zhejiang)

Zanthoxylum simulans Hance subsp. calcareum Z. H. Chen, F. Chen \& W. Zhu 毛野花椒 [New Subspecies]

CHEN Feng, XIE Wen-yuan, ZHANG Shui-li, ZHANG Fen-yao, CHEN Zheng-hai, Additional Notes on the Seed Plants in Zhejiang (II). Journal of Hangzhou Normal University (Natural Science Edition) 19 (6): 96-102. 2020 [100] doi: 10.12191/j.issn.1674232X.2020.06.014

CHINA (Zhejiang*)

\section{Salicaceae 杨柳科}

Salix acuminatomicrophylla K. S. Hao ex C. F. Fang \& A. K. Skvortsov [Typification] 
杜诚, 刘军, 叶文, 廖帅, 葛斌杰, 刘冰, 马金双 (2021) 中国植物新分类群、新名称 2020 年度报告. 生物多样性, 29, 10111020. http://www.biodiversity-science.net/CN/10.17520/biods.2021122

HE Li, APPLEQUIST Wendy L., Lectotypifications and Nomenclatural Notes for Hao's New Chinese Taxa in Salix (Salicaceae). Novon 28 (3): 180-185. 2020 [180] doi: 10.3417/2020602

CHINA (Yunnan*)

Salix alfredii Goerz ex Rehder \& Kobuski [Typification]

LIU Li-juan, HE Li, APPLEQUIST Wendy L., Untangling Two Chinese Salix Species (Salicaceae) Published by C. K. Schneider, with Lectotypification of Four Names. Willdenowia 50 (2): 159-163. 2020 [161] doi: 10.3372/wi.50.50201

CHINA (Gansu*)

Salix annulifera C. Marquand \& Airy Shaw 环纹矮柳 [Typification]

ZENG Si-wen, HE Li, Lectotypification and Morphological Notes of Salix annulifera C. Marquand \& Airy Shaw (Salicaceae). Journal of Tropical and Subtropical Botany 28 (3): 236-240. 2020 [237] doi: 10.11926/jtsb.4106

CHINA (Xizang*, Yunnan)

Salix atopantha C. K. Schneid. var. glabra K. S. Hao ex C. F. Fang \& A. K. Skvortsov [Typification]

HE Li, APPLEQUIST Wendy L., Lectotypifications and Nomenclatural Notes for Hao's New Chinese Taxa in Salix (Salicaceae). Novon 28 (3): 180-185. 2020 [181] doi: 10.3417/2020602

CHINA (Zhejiang*)

Salix crenata K. S. Hao [Typification]

HE Li, APPLEQUIST Wendy L., Lectotypifications and Nomenclatural Notes for Hao's New Chinese Taxa in Salix (Salicaceae). Novon 28 (3): 180-185. 2020 [181] doi: 10.3417/2020602

CHINA (Yunnan*)

Salix forrestii K. S. Hao ex C. F. Fang \& A. K. Skvortsov [Typification]

HE Li, APPLEQUIST Wendy L., Lectotypifications and Nomenclatural Notes for Hao's New Chinese Taxa in Salix (Salicaceae). Novon 28 (3): 180-185. 2020 [182] doi: 10.3417/2020602

CHINA (Yunnan*)

Salix kansuensis K. S. Hao ex C. F. Fang \& A. K. Skvortsov [Typification]

HE Li, APPLEQUIST Wendy L., Lectotypifications and Nomenclatural Notes for Hao's New Chinese Taxa in Salix (Salicaceae). Novon 28 (3): 180-185. 2020 [182] doi: 10.3417/2020602

CHINA (Gansu*)

Salix mictotricha C. K. Schneid. [Typification]

LIU Li-juan, HE Li, APPLEQUIST Wendy L., Untangling Two Chinese Salix Species (Salicaceae) Published by C. K. Schneider, with Lectotypification of Four Names. Willdenowia 50 (2): 159-163. 2020 [161] doi: 10.3372/wi.50.50201

CHINA (Hubei*)

Salix ovatomicrophylla K. S. Hao ex C. F. Fang \& A. K. Skvortsov [Typification]

HE Li, APPLEQUIST Wendy L., Lectotypifications and Nomenclatural Notes for Hao's New Chinese Taxa in Salix (Salicaceae). Novon 28 (3): 180-185. 2020 [183] doi: 10.3417/2020602

CHINA (Yunnan*)

Salix polyclona C. K. Schneid. [New Synonyms]

S.: Salix mictotricha C. K. Schneid.; Salix alfredii Goerz ex Rehder \& Kobuski; Salix wuana K. S. Hao ex C. F. Fang \& A. K. Skvortsov LIU Li-juan, HE Li, APPLEQUIST Wendy L., Untangling Two Chinese Salix Species (Salicaceae) Published by C. K. Schneider, with Lectotypification of Four Names. Willdenowia 50 (2): 159-163. 2020 [161] doi: 10.3372/wi.50.50201

CHINA (Gansu, Henan, Hubei*, Qinghai, Shaanxi, Sichuan)

Salix polyclona C. K. Schneid. [Typification]

LIU Li-juan, HE Li, APPLEQUIST Wendy L., Untangling Two Chinese Salix Species (Salicaceae) Published by C. K. Schneider, with Lectotypification of Four Names. Willdenowia 50 (2): 159-163. 2020 [161] doi: 10.3372/wi.50.50201

CHINA (Gansu, Henan, Hubei*, Qinghai, Shaanxi, Sichuan)

Salix pseudowolohoensis K. S. Hao ex C. F. Fang \& A. K. Skvortsov [Typification]

HE Li, APPLEQUIST Wendy L., Lectotypifications and Nomenclatural Notes for Hao's New Chinese Taxa in Salix (Salicaceae). Novon 28 (3): 180-185. 2020 [183] doi: 10.3417/2020602

CHINA (Yunnan*)

Salix rhoophila C. K. Schneid. [Typification]

LIU Li-juan, HE Li, APPLEQUIST Wendy L., Untangling Two Chinese Salix Species (Salicaceae) Published by C. K. Schneider, with Lectotypification of Four Names. Willdenowia 50 (2): 159-163. 2020 [160] doi: 10.3372/wi.50.50201

CHINA (Hubei*, Shaanxi) 
杜诚, 刘军, 叶文, 廖帅, 葛斌杰, 刘冰, 马金双 (2021) 中国植物新分类群、新名称 2020 年度报告. 生物多样性, 29, 10111020. http://www.biodiversity-science.net/CN/10.17520/biods.2021122

Salix schneideriana K. S. Hao ex C. F. Fang \& A. K. Skvortsov [Typification]

HE Li, APPLEQUIST Wendy L., Lectotypifications and Nomenclatural Notes for Hao's New Chinese Taxa in Salix (Salicaceae). Novon 28 (3): 180-185. 2020 [183] doi: 10.3417/2020602

CHINA (Yunnan*)

\section{Saxifragaceae 虎耳草科}

Chrysosplenium pilosopetiolatum C. P. Tsien 毛柄金腰 [New Synonyms]

S.: Chrysosplenium pilosum Maxim. var. pilosopetiolatum (C. P. Tsien) J. T. Pan

CHEN Feng, XIE Wen-yuan, ZHANG Fen-yao, ZHANG Hong-wei, CHEN Xian-xing, CHEN Zheng-hai, Notes on Seed Plant in Zhejiang Province (V). Journal of Zhejiang Forestry Science and Technology 40 (4): 67-72. 2020 [68] doi: 10.3969/j.issn.10013776.2020.04.012

CHINA (Hunan*, Zhejiang)

Chrysosplenium zhouzhiense Hong Liu bis 周至金腰 [New Species] IUCN: EN: D1; Evidence: Morphology

FU Long-fei, LIAO Rui, LAN De-qing, WEN Fang, LIU Hong, A New Species of Chrysosplenium (Saxifragaceae) from Shaanxi, North-western China. PhytoKeys 159: 127-135. 2020 [129] doi: 10.3897/phytokeys.159.56109

CHINA (Shaanxi*)

\section{Scapaniaceae 合叶苔科}

Diplophyllum purpurascens Bakalin \& Vilnet [New Species] IUCN: NE; Evidence: Morphology

BAKALIN Vadim, VILNET Anna, KLIMOVA Ksenia, MA Wen-zhang, NGUYEN Van Sinh, Diplophyllum purpurascens (Scapaniaceae, Marchantiophyta) - a New Species from Sino-Himalaya (China). Phytotaxa 447 (2): 116-126. 2020 [120] doi: 10.11646/phytotaxa.447.2.4

CHINA (Yunnan*)

\section{Selaginellaceae 卷柏科}

Selaginella subvaginata X. C. Zhang \& Shalimov [New Species] IUCN: DD; Evidence: rbcL, atpI, psbA; Morphology

ZHANG Xian-chun, SHALIMOV Aleksandr Petrovich, KANG Jong-soo, ZHANG Meng-hua, Selaginella subvaginata (Selaginellaceae), a New Spikemoss from China. Journal of Species Research 9 (3): 221-232. 2020 [222] doi: 10.12651/JSR.2020.9.3.221

CHINA (Sichuan *)

\section{Solanaceae 茄科}

Solanum triflorum Nutt. 羽裂叶龙葵 [New Record]

YAN Jing, ZHAO Li-qing, MA Jin-shuang, Solanum triflorum Nutt., a Newly Naturalized Species of Solanum in China. Plant Science Journal 38 (6): 730-732. 2020 [731] doi: 10.11913/PSJ.2095-0837.2020.60730

CHINA (Nei Mongol); UNITED STATES*

\section{Styracaceae 安息香科}

Melliodendron wangianum Hu [Typification]

WU Xue-hui, ZHANG Rui, UTTERIDGE Timothy M. A., LIU Yu-lin, YANG Guang-Yao, TANG Ming, A Review of the Chinese Monotypic Genus Melliodendron (Styracaceae), with a New Synonym of M. xylocarpum. Kew Bulletin 75 (53): 1-8. 2020 [3] doi: 10.1007/S12225-020-09913-4

CHINA (Sichuan*)

Melliodendron xylocarpum Hand.-Mazz. [New Synomyms]

S.: Melliodendron xylocarpum Hand.-Mazz. var. roseolum Z. X. Yu

WU Xue-hui, ZHANG Rui, UTTERIDGE Timothy M. A., LIU Yu-lin, YANG Guang-Yao, TANG Ming, A Review of the Chinese Monotypic Genus Melliodendron (Styracaceae), with a New Synonym of M. xylocarpum. Kew Bulletin 75 (53): 1-8. 2020 [3] doi: 10.1007/S12225-020-09913-4

CHINA (Fujian, Guangdong*, Guangxi, Guizhou, Hong Kong, Hunan, Jiangxi, Shanghai, Sichuan, Yunnan)

Melliodendron xylocarpum Hand.-Mazz. var. roseolum Z. X. Yu [Typification]

WU Xue-hui, ZHANG Rui, UTTERIDGE Timothy M. A., LIU Yu-lin, YANG Guang-Yao, TANG Ming, A Review of the Chinese Monotypic Genus Melliodendron (Styracaceae), with a New Synonym of M. xylocarpum. Kew Bulletin 75 (53): 1-8. 2020 [3] doi: 10.1007/S12225-020-09913-4

CHINA (Jiangxi*) 
杜诚, 刘军, 叶文, 廖帅, 葛斌杰, 刘冰, 马金双 (2021) 中国植物新分类群、新名称 2020 年度报告. 生物多样性, 29, 10111020. http://www.biodiversity-science.net/CN/10.17520/biods.2021122

Rehderodendron macrophyllum (C. Y. Wu \& K. M. Feng) W. Y. Zhao, P. W. Fritsch \& W. B. Liao 大叶木瓜红 [New Combination] B.: Parastyrax macrophyllus C. Y. Wu \& K. M. Feng

ZHAO Wan-yi, FRITSCH Peter W., LIU Zhong-cheng, FAN Qiang, JIN Jian-hua, LIAO Wen-bo, New Combinations and Synonyms in Rehderodendron (Styracaceae). PhytoKeys 161: 79-88. 2020 [84] doi: 10.3897/phytokeys.161.54970

CHINA (Yunnan*)

Rehderodendron macrophyllum (C. Y. Wu \& K. M. Feng) W. Y. Zhao, P. W. Fritsch \& W. B. Liao 大叶木瓜红 [Typification]

ZHAO Wan-yi, FRITSCH Peter W., LIU Zhong-cheng, FAN Qiang, JIN Jian-hua, LIAO Wen-bo, New Combinations and Synonyms in Rehderodendron (Styracaceae). PhytoKeys 161: 79-88. 2020 [84] doi: 10.3897/phytokeys.161.54970

CHINA (Yunnan*)

Rehderodendron microcarpum K. M. Feng ex T. L. Ming [New Synonyms]

S.: Rehderodendron gongshanense Y. C. Tang

ZHAO Wan-yi, FRITSCH Peter W., FAN Qiang, LIAO Wen-bo, Taxonomic Reassessment of Rehderodendron gongshanense

(Styracaceae) Based on Herbarium Specimens and Field Observations. Phytotaxa 450 (1): 1-7. 2020 [3] doi:

10.11646/phytotaxa.450.1.1

CHINA (Yunnan*); MYANMAR

Styrax buchananii W. W. Sm 中缅安息香 [New Record]

ZHAO Wan-yi, LIU Zhong-cheng, JIN Jian-hua, LIAO Wen-bo, Styrax buchananii W. W. Sm., A Newly Recorded Species of

Styracaceae from China. Journal of Tropical and Subtropical Botany 28 (5): 520-522. 2020 [520] doi: 10.11926/jtsb.4243

CHINA (Yunnan); MYANMAR*

\section{Theaceae 山茶科}

Camellia hainanica Y. L. Zhao \& Z. G. Xu [New Species] IUCN: NE; Evidence: matK; Morphology

XU Zheng-gang, YUAN De-yi, TANG Yong-cheng, WU Liang, ZHAO Yun-lin1, Camellia hainanica (Theaceae) a New Species from Hainan, Supported from Morphological Characters and Phylogenetic Analysis. Pakistan Journal of Botany 52 (3): 1025-1032. 2020 [1028] doi: 10.30848/PJB2020-3(31)

CHINA (Hainan*)

Camellia lucidissima Hung T. Chang subsp. jingningensis Z. H. Chen, P. L. Chiu \& W. Y. Xie 景宁白山茶 [New Subspecies]

XIE Wen-yuan, CHEN Feng, ZHANG Fen-yao, XU Shao-qing, CHEN Zheng-hai, Correction and Supplement of the Seed Plant Flora in Zhejiang. Journal of Hangzhou Normal University (Natural Science Edition) 19 (3): 247-252. 2020 [250] doi: 10.12191/j.issn.1674-232X.2020.03.005

CHINA (Zhejiang*)

Camellia microphylla (Merr.) S. S. Chien 细叶短柱茶 [New Synomyms]

S.: Camellia brevistyla Cohen-Stuart var. microphylla (Merr.) T. L. Ming

XIE Wen-yuan, CHEN Feng, ZHANG Fen-yao, XU Shao-qing, CHEN Zheng-hai, Correction and Supplement of the Seed Plant Flora in Zhejiang. Journal of Hangzhou Normal University (Natural Science Edition) 19 (3): 247-252. 2020 [249] doi: 10.12191/j.issn.1674-232X.2020.03.005

CHINA (Anhui*)

Camellia microphylla (Merr.) S. S. Chien f. rubida (P. L. Chiu) Z. H. Chen \& P. L. Chiu 红花短柱茶 [New Combination]

B.: Camellia brevistyla Cohen-Stuart f. rubida P. L. Chiu

XIE Wen-yuan, CHEN Feng, ZHANG Fen-yao, XU Shao-qing, CHEN Zheng-hai, Correction and Supplement of the Seed Plant Flora in Zhejiang. Journal of Hangzhou Normal University (Natural Science Edition) 19 (3): 247-252. 2020 [250] doi: 10.12191/j.issn.1674-232X.2020.03.005

CHINA (Zhejiang*)

Camellia rostrata S. X. Yang \& S. F. Chai 㗒果金花茶 [New Species] IUCN: CR; Evidence: Morphology

LIU Zhen-wen, CHAI Sheng-feng, WU Fang-yuan, YE Pin-ming, JIANG Chang-jie, ZHANG Zhi-rong, YU Xiang-qin, MA Jin-lin, YANG Shi-xiong, Camellia rostrata, a New Species of Yellow Camellias from Southwest China. Phytotaxa 459 (1): 61-68. 2020 [62] doi: 10.11646/phytotaxa.459.1.6

CHINA (Guangxi*)

Camellia zhaiana S. X. Yang 红花长柄茶 [New Species] IUCN: NE; Evidence: Morphology

LIU Zhen-wen, YE Pin-ming, LI Zhi-hui, WEI Xiao-juan, LIU En-de, ZHANG Zhi-rong, MA Jin-lin, YANG Shi-xiong, Camellia zhaiana (sect. Longipedicellata), a New Species of Theaceae from Guangxi, China. Phytotaxa 460 (3): 225-229. 2020 [225] doi: 10.11646/phytotaxa.460.3.5

CHINA (Guangxi*) 
杜诚, 刘军, 叶文, 廖帅, 葛斌杰, 刘冰, 马金双 (2021) 中国植物新分类群、新名称 2020 年度报告. 生物多样性, 29, 10111020. http://www.biodiversity-science.net/CN/10.17520/biods.2021122

Stewartia gemmata S. S. Chien \& W. C. Cheng 天目紫茎 [New Synonyms]

S.: Stewartia brevicalyx S. Z. Yan; Stewartia sinensis Rehder \& E. H. Wilson var. brevicalyx (S. Z. Yan) T. L. Ming \& J. Li

CHEN Feng, XIE Wen-yuan, ZHANG Fen-yao, ZHANG Hong-wei, CHEN Xian-xing, CHEN Zheng-hai, Notes on Seed Plant in Zhejiang Province (V). Journal of Zhejiang Forestry Science and Technology 40 (4): 67-72. 2020 [69] doi: 10.3969/j.issn.10013776.2020.04.012

CHINA (Zhejiang*)

Stewartia gemmata S. S. Chien \& W. C. Cheng 天目紫茎 [Typification]

CHEN Feng, XIE Wen-yuan, ZHANG Fen-yao, ZHANG Hong-wei, CHEN Xian-xing, CHEN Zheng-hai, Notes on Seed Plant in Zhejiang Province (V). Journal of Zhejiang Forestry Science and Technology 40 (4): 67-72. 2020 [69] doi: 10.3969/j.issn.10013776.2020.04.012

CHINA (Zhejiang*)

\section{Thymelaeaceae 瑞香科}

Daphne kiusiana Miq. f. purpurea X. F. Jin, Z. H. Chen \& Y. F. Lu 红花毛瑞香 [New Form]

ZHANG Zhong-zhao, CHEN Zheng-hai, CHEN Feng, XIE Wen-yuan, LI Gen-you, Additional Notes on the Seed Plant Flora of Zhejiang( VII ). Journal of Zhejiang Forestry Science and Technology 40 (6): 52-55. 2020 [54] doi: 10.3969/j.issn.10013776.2020.06.008

CHINA (Zhejiang*)

\section{Vitaceae 葡萄科}

Causonis wentsiana Z. H. Chen, F. Chen \& X. F. Jin [New Species] IUCN: NE; Evidence: atpB-rbcL, trnC-petN, trnH-psbA, trnL-F; Morphology

CHEN Zheng-hai, CHEN Feng, LU Yi-fei, XIE Wen-yuan, JIN Xiao-feng, Three New Species of Cayratia-like genera (Vitaceae) from Zhejiang, East China. Phytotaxa 475 (4): 253-266. 2020 [257] doi: 10.11646/phytotaxa.475.4.3

CHINA (Zhejiang*)

Causonis montana Z. H. Chen, Y. F. Lu \& X. F. Jin [New Species] IUCN: NE; Evidence: atpB-rbcL, trnC-petN, trnH-psbA, trnL-F; Morphology

CHEN Zheng-hai, CHEN Feng, LU Yi-fei, XIE Wen-yuan, JIN Xiao-feng, Three New Species of Cayratia-like genera (Vitaceae) from Zhejiang, East China. Phytotaxa 475 (4): 253-266. 2020 [261] doi: 10.11646/phytotaxa.475.4.3

CHINA (Zhejiang*)

Pseudocayratia orientalisinensis Z. H. Chen, W. Y. Xie \& X. F. Jin [New Species] IUCN: NE; Evidence: atpB-rbcL, trnC-petN, trnHpsbA, trnL-F; Morphology

CHEN Zheng-hai, CHEN Feng, LU Yi-fei, XIE Wen-yuan, JIN Xiao-feng, Three New Species of Cayratia-like genera (Vitaceae) from Zhejiang, East China. Phytotaxa 475 (4): 253-266. 2020 [261] doi: 10.11646/phytotaxa.475.4.3

CHINA (Zhejiang*)

\section{Woodsiaceae 岩嬞科}

Physematium indusiosum (Christ) Li Bing Zhang, N. T. Lu \& X. F. Gao [New Combination]

B.: Woodsia indusiosa Christ

LU Ngan Thi, ZHOU Xin-Mao, ZHANG Liang, KNAPP Ralf, LI Chun-xiang, FAN Xue-ping, ZHOU Lin, WEI Hong-jin, LU Jin-mei, XU Bo, PENG Yu-lan, GAO Xin-fen, ZHANG Li-bing, A Global Plastid Phylogeny of the Cliff Fern Family Woodsiaceae and a Two-Genus Classification of Woodsiaceae with the Description of $\times$ Woodsimatium nothogen. nov. Taxon 68 (6): 1149-1172. 2020 [1166] doi: 10.1002/tax.12180

CHINA (Yunnan)

Physematium kangdingense (H. S. Kung, Li Bing Zhang \& X. S. Guo) Li Bing Zhang, N. T. Lu \& X. F. Gao [New Combination]

B.: Woodsia kangdingensis H. S. Kung, Li Bing Zhang \& X. S. Guo

LU Ngan Thi, ZHOU Xin-Mao, ZHANG Liang, KNAPP Ralf, LI Chun-xiang, FAN Xue-ping, ZHOU Lin, WEI Hong-jin, LU Jin-mei, XU Bo, PENG Yu-lan, GAO Xin-fen, ZHANG Li-bing, A Global Plastid Phylogeny of the Cliff Fern Family Woodsiaceae and a Two-Genus Classification of Woodsiaceae with the Description of $\times$ Woodsimatium nothogen. nov. Taxon 68 (6): 1149-1172. 2020 [1166] doi: 10.1002/tax.12180

CHINA (Sichuan, Yunnan)

\section{Zingiberaceae 姜科}

Boesenbergia quangngaiensis N. S. Lý 广义凹唇姜 [New Record] 
杜诚, 刘军, 叶文, 廖帅, 葛斌杰, 刘冰, 马金双 (2021) 中国植物新分类群、新名称 2020 年度报告. 生物多样性, 29, 10111020. http://www.biodiversity-science.net/CN/10.17520/biods.2021122

LI Wei-jie, YANG Hai-jian, YE Yu-shi, KANG Yong, ZHANG Wen, JIANG Xi-ling, PANG Yu-xin, ZHENG Xi-long, Boesenbergia quangngaiensis N. S. Lý, A Newly Recorded Species o Zingiberaceae from China. Journal of Tropical and Subtropical Botany 28 (3): 241-244. 2020 [241] doi: 10.11926/jtsb.4153

CHINA (Hainan); VIETNAM*

Hedychium mechukanum M. Sabu \& Hareesh [New Species] IUCN: DD; Evidence: Morphology

SABU M., HAREESH V. S., Hedychium mechukanum (Zingiberaceae), A New Species from the Eastern Himalayas, India. The Gardens' Bulletin Singapore 72 (2): 291-297. 2020 [292] doi: 10.26492/gbs72(2).2020-12

CHINA (Xizang*)

Zingiber chengii Y. H. Tseng, C. M. Wang \& Yuan C. Lin 狭叶姜 [New Species] IUCN: EN: B1;C2a(i); Evidence: Morphology

WANG Chiu-mei, LIN Yuan-chien, TSENG Yen-hsueh, Zingiber chengii (Zingiberaceae), a New Species from Taiwan. PhytoKeys 139: 1-11. 2020 [3] doi: 10.3897/phytokeys.139.37294

CHINA (Taiwan*)

Zingiber porphyrochilum Y. H. Tan \& H. B. Ding [New Species] IUCN: NE; Evidence: Morphology

DING Hong-bo, YANG Bin, LU Xiao-qiang, TAN Yun-hong, Zingiber porphyrochilum (Zingiberaceae), a New Species from Yunnan, China. Annales Botanici Fennici 57 (4-6): 197-201. 2020 [197] doi: 10.5735/085.057.0401

CHINA (Yunnan*) 
杜诚, 刘军, 叶文, 廖帅, 葛斌杰, 刘冰, 马金双 (2021) 中国植物新分类群、新名称 2020 年度报告. 生物多样性, 29, 10111020. http://www.biodiversity-science.net/CN/10.17520/biods.2021122

\section{List of journals}

Acta Botanica Boreali-Occidentalia Sinica, 西北植物学报, 西北植物研究 (1981-1984). ISSN: 1000-4025. China: Yangling 1981-.

Acta Bryolichenologica Asiatica，亚洲苔蘚地衣学报. ISSN: 1016-6181. Finland: Mailantie 1989-

Acta Phytotaxonomica \& Geobotanica. ISSN: 1346-7565. Japan: Tokyo 2001-. Continues: 植物分类，地理（1932-2001).

Annales Botanici Fennici. ISSN: 0003-3847 [P]/ 1797-2442 [E]. Finland: Helsinki 1964-.

Annals of the Missouri Botanical Garden. ISSN: 0026-6493 [P]/ 2162-4372 [E]. United States: St. Louis 1914-.

Bangladesh Journal of Botany. ISSN: 0253-5416 [P]/ 2079-9926 [E]. Bangladesh: Dhaka 1972-.

Biodiversity Science, 生物多样性, Chinese Biodiversity. ISSN: 1005-0094. China: Beijing 1993-.

Botanical Studies (Taipei), a Springer Open Journal，植物学研究. ISSN: 1999-3110. China, Taiwan: Taipei 2013-.Continues: Botanical Bulletin of Academia Sinica “中央研究院” 植物学汇刊 (1960-2005), Botanical Studies a International Journal (2006-2012).

Brittonia. ISSN: 0007-196X [P]/ 1938-436X [E]. United States: New York 1931-.

Bulletin of Botanical Research，植物研究，木本植物研究 (1988-2000). ISSN: 1673-5102. China: Harbin 1980-. Continues: Bulletin of the Hebarium of North-Eastern Forestry Academy 东北林学院植物标本室刊 (1959-1961), Bulletin of Botanical Laboratory of Norestry Instite 东北林学院植物研究室汇刊 (1979-1980).

Bulletin of the Cupressus Conservation Project. ISSN: 2235-3992 [P]/ 2235-400X [E]. Switzerland: Geneva 2012-.

Cladistics, The International Journal of the Willi Hennig Society. ISSN: 0748-3007 [P]/ 1096-0031 [E]. United States: Westport 1985-.

Die Orchidee (Herausgeber). ISSN: 2366-0643 [E]. Germany: Niedernhausen 2015-. Continues: Die Orchidee, Beiträge zur Förderung der Orchideenkunde. ISSN: 0473-1425. Germany: Niedernhausen 1949-2014.

Forest Science and Technology，林业科技通讯. ISSN: 1671-4938. China: Beijing 1958-.

Frahmia. ISSN: 2199-4897. Germany: Bernhausen 2014-.

Frontiers in Plant Science. ISSN: 1664-462X [E]. Switzerland: Lausanne 2010-.

Guihaia, 广西植物. ISSN: 1000-3142. China: Guilin 1980-. Continues: 植物研究通讯 内刊 (1975-1978).

Indian Forester. ISSN: 0019-4816 [P]/ 2321-094X [E]. India: Dehradun 1875-.

Hattoria. ISSN: 2185-8241 [P]/ 2423-8961 [E]. Japan: Nichinan 2010-

Herzogia: Zeitschrift der Bryologisch-licheologischen Arbeitsgemeinschaft für Mitteleuropa. ISSN: 0018-0971 [P]/ $2700-2829$ [E]. Germany: Lehre 1968-

Inner Mongolia Forestry Investigation and Design, 内蒙古林业调查设计. ISSN: 1006-6993. China: Hohhot 1978-.

International Journal of Plant Sciences. ISSN: 1058-5893 [P]/ 1537-5315 [E]. United States: Chicago 1992-. Continues: Botanical Gazette (Chicago, Ill.) (1876-1991).

Journal of Botanical Research. ISSN: 2630-5054 [E]. Singapore: Singapore 2019-.

Journal of Bryology. ISSN: 0373-6687 [P]/ 1743-2820 [E]. United Kingdom: Leeds 1972-. Continues: Transactions of the British Bryological Society (1947-1971).

Journal of Fujian Agriculture and Forestry University (Natural Science Edition), 福建农林大学学报 (自然科学版). ISSN: 16715470. China: Fuzhou. 1997-. Continues: Journal of Fujian Agricultural University 福建农业大学学报 (1995-1996); Journal of Fujian Agricultural College 福建农学院学报 (1953-1994). 
杜诚, 刘军, 叶文, 廖帅, 葛斌杰, 刘冰, 马金双 (2021) 中国植物新分类群、新名称 2020 年度报告. 生物多样性, 29, 10111020. http://www.biodiversity-science.net/CN/10.17520/biods.2021122

Journal of Hangzhou Normal University (Natural Science Edition), 杭州师范大学学报 (自然科学版). ISSN: 1674-232X. China: Hangzhou 2008-. Continues: Journal of Hangzhou Teachers College (Natural Sciences Edition) 杭州师范学院学报 (自然科学版) (1979-2007).

Journal of Liaoning Normal University (Natural Science Edition), 辽宁师范大学学报 (自然科学版). ISSN: 1000-1735. China: Dalian 1984-. Continues: Journal of Liaoning Normal College (Natural Science Edition) 辽宁师院学报 (自然科学版) (1978-1983)

Journal of Nanjing Forestry University (Natural Sciences Edition), 南京林业大学学报 (自然科学版). ISSN: 1000-2006. China: Nanjing 1986-. Continues: Nanjing Forestry College (1958-1971, 1983-1985) 南京林学院学报, Nanjing Technological College of Forest Products 南京林产工学院学报 (1972-1982).

Journal of Plant Resources and Environment, 植物资源与环境学报，植物资源与环境 (1992-1999). ISSN:1674-7895. China: Nanjing 1992-.

Journal of Species Research. ISSN: 2234-7909. Korea: Incheon 2012-.

Journal of Systematics and Evolution. ISSN: 1674-4918 [P]/ 1759-6831 [E]. China: Beijing 2009-. Continues: Acta Phytotaxonomica Sinica 植物分类学报 (1951-2008).

Journal of Tropical and Subtropical Botany，热带亚热带植物学报. ISSN: 1005-3395. China: Guangzhou 1993-. Continues: 华南 植物研究 (1992).

Journal of Wenzhou University (Natural Science Edition), 温州大学学报 (自然科学版). ISSN: 1674-3563. China: Wenzhou 2007-. Continues: 温州师范学院学报 (1987-2006); 温州师专学报 (1980-1986); 温州师范学院学报 (1963).

Journal of West China Forestry Science, 西部林业科学. ISSN: 1672-8246. China: Kunming 2004-. Continues: Yunnan Forestry Science and Technology 云南林业科技 (1972-2003).

Journal of Zhejiang Forestry Science and Technology，浙江林业科技. ISSN: 1001-3776. China: Hangzhou 1972-.

Journal of Zhejiang University (Science Edition), 浙江大学学报 (理学版). ISSN: 1008-9497. China: Hangzhou 1999-. Continues: Journal of Hangzhou University (Nature Science) 杭州大学学报 (自然科学版) (1956-1998).

Kew Bulletin. ISSN: 0075-5974 [P]/ 1874-933X [E]. United Kingdom: Surrey 1946-.

Molecular Phylogenetics and Evolution. ISSN: 1055-7903 [P]/ 1095-9513 [E]. United States: Orlando 1993-.

Nordic Journal of Botany. ISSN: 1756-1051. Sweden: Oxford 1980-. Continues: Norwegian Journal of Botany (1971-1980), Nytt Magasin for Botanikk (1952-1970).

Northern Horticlture，北方园艺. ISSN: 1001-0009. China: Harbin 1987-. Continues：黑龙江园艺 (1977-1986).

Novon, a Journal for Botanical Nomenclature. ISSN: 1055-3177 [P]/ 1945-6174 [E]. United States: St. Louis 1991-.

Novosti Sistematiki Vysshikh Rasteniĭ, Новости Систематики Высших Растений. ISSN: 0568-5435. Russia: St. Petersburg $1964-$.

Pakistan Journal of Botany. ISSN: 0556-3321 [P]/ 2070-3368 [E]. Pakistan: Peshawar 1995-.

PhytoKeys. ISSN: 1314-2011 [P]/ 1314-2003 [E]. Bulgaria: Sofia 2010-.

Phytoneuron, Digital Publications in Plant Biology. ISSN: 2153-733X. United States: Fort Worth 2010-.

Phytotaxa. ISSN: 1179-3155 [P]/ 1179-3163 [E]. New Zealand: Auckland 2009-.

Plant Ecology and Evolution. ISSN: 2032-3913 [P]/ 2032-3921 [E]. Belgique: Brussels 2010-.

Plant Diversity. ISSN: 2468-2659. China: Kunming 2016-. Continues: 云南植物研究内刊 (1975-1978), Acta Botanica Yunnanica 云南植物研究 (1979-2010), Plant Diversity and Resources 植物分类与资源学报 (2011-2015).

Plant Quarantine, 植物检疫. ISSN: 1005-2755. China: Beijing 1979-.

Plant Science Journal, 植物科学学报. ISSN: 2095-0837. China: Wuhan 2011-. Continues: Journal of Wuhan Botanical Research 武 汉植物研究 (1983-2010). 
杜诚, 刘军, 叶文, 廖帅, 葛斌杰, 刘冰, 马金双 (2021) 中国植物新分类群、新名称 2020 年度报告. 生物多样性, 29, 10111020. http://www.biodiversity-science.net/CN/10.17520/biods.2021122

Plant Systematics and Evolution. ISSN: 0378-2697 [P]/ 2199-6881 [E]. Austria: Wien 1974-. Continues: Oesterreichische Botanische Zeitschrift (1858-1942), (1947-1996).

Plants (MDPI). ISSN: 2223-7747. Switzerland: Basel 2012-.

Protection Forest Science and Technology，防护林科技. ISSN: 1005-5215. China: Qiqihar 1983-.

Rheedea, Official Journal of Indian Association for Angiosperm Taxonomy. ISSN: 0971-2313. India: Kolkata 1991-.

Symbolae Botanicae Upsalienses, Acta Universitatis Upsaliensis. ISSN: 0082-0644. Sweden: Uppsala 1932-.

Systematic Botany, Quarterly Journal of the American Society of Plant Taxonomists. ISSN: 0363-6445 [P]/ $1548-2324$ [E]. United States: New York 1976-. Continues: Brittonia (1937-1975).

Taiwan Journal of Biodiversity，台湾生物多样性研究. ISSN: 2076-6971. China, Taiwan: Nantou 2010-. Continues: Endemic Species Research 特有生物研究 (1999-2009).

Taiwan Quarterly Journal of Forest Research, 林业研究季刊. ISSN: 1606-8351. China, Taiwan: Taichung 1979-.

Taiwania, International Journal of Life Sciences. ISSN: 0372-333X. China, Taiwan: Taipei 1948-. Continues: Acta Botanica Taiwanica (1947).

Taxon, International Journal of Taxonomy, Phylogeny and Evolution. ISSN: 0040-0262 [P]/ 1996-8175 [E]. International Organization: Bratislava 1951-.

Thai Forest Bulletin (Botany). ISSN: 0495-3843. Thailand: Bangkok 1954-.

The Gardens’ Bulletin Singapore. ISSN: 0374-7859 [P]/ 2382-5812 [E]. Singapore: Singapore 1967-. Continues: The Gardens' Bulletin, Straits Settlements (1912-1966).

The Journal of Japanese Botany, 植物研究杂志. ISSN: 0022-2062. Japan: Tokyo 1916-.

Willdenowia, Mitteilungen aus dem Botanischen Garten und Museum Berlin-Dahlem. ISSN: 0511-9618. Germany: Berlin-Dahlem 1953-.

World Bamboo and Rattan，世界竹藤通讯. ISSN: 1672-0431. China: Beijing 2003-. Continues: 竹类文摘 (1988-2002) 


\section{Types of Chinese new plant species in 2020}

Aconitum haridasanii R. Tiwary, Harsh Singh \& D. Adhikari (Ranunculaceae) Phytotaxa 440 (3): 232. 2020.

TYPE: CHINA (Xizang*), 9/20/2018, R. Tiwary, H. Singh, P. P. Singh \& D. Adhikari 303752 (holotype: LWG; isotype: LWG).

Aconitum laxifoliatum W. T. Wang (Ranunculaceae) Bulletin of Botanical Research 40 (1): 4. 2020.

TYPE: CHINA (Sichuan*), 9/10/2018, S. R. Zhang, Y. M. Zhao \& J. X. Wang 1766 (holotype: PE-02293706).

Aconitum tenuigaleatum W. T. Wang (Ranunculaceae) Bulletin of Botanical Research 40 (1): 1. 2020.

TYPE: CHINA (Sichuan*), 9/11/2018, S. R. Zhang, Y. M. Zhao \& Z. S. Zhang 1682 (holotype: PE-02293704).

Ainsliaea daheishanensis Y. L. Peng, C. X. Yang \& Y. Luo (Asteraceae) PhytoKeys 138: 234. 2020.

TYPE: CHINA (Yunnan*), 1/14/2019, Y. L. Peng \& C. X. Yang SE02248 (holotype: CDBI; isotype: HITBC).

Ainsliaea wuyuanensis Z. H. Chen, Y. L. Xu \& X. F. Jin (Asteraceae) Guihaia 40 (1): 96.2020.

TYPE: CHINA (Jiangxi*), 10/11/2018, Z. H. Chen, F. Chen \& Y. H. Hong JXWY18101102 (holotype: ZM; isotype: ZM).

Alangium confertiflorum Y. H. Tan \& H. B. Ding (Cornaceae) Taiwania 65 (4): 517. 2020.

TYPE: CHINA (Yunnan*), LAOS, 4/23/2019, Y. H. Tan, B. Yang, H. B. Ding, X. D. Zeng, D. L. Quan, G. Yan \& S. H. You T0456 (holotype: HITBC-0030007; isotype: HITBC-0030008, HITBC-0030009); B. Yang \& al. T0457 (paratype: HITBC-0030010, HITBC0030011, HITBC-0030012); J. W. Li \& Y. H. Tan 3019 (paratype: HITBC, TAI); J. W. Li 4548 (paratype: HITBC, KUN, K); Y. H. Tan \& al. G2-0046 (paratype: HITBC); Y. H. Tan \& al. (paratype: HITBC); B. Yang \& al. G2-373 (paratype: HITBC); Y. H. Tan \& al. L0988 (paratype: HITBC, HNL).

Allium xinlongense D. F. Xie \& X. J. He (Amaryllidaceae) Phytotaxa 432 (3): 280. 2020.

TYPE: CHINA (Sichuan*), 9/23/2019, D. F. Xie XDF20190923 (holotype: SZ; isotype: SZ).

Allocheilos maguanensis W. H. Chen \& Y. M. Shui (Gesneriaceae) PhytoKeys 157: 158. 2020.

TYPE: CHINA (Yunnan*), 7/11/2017, Y. M. Shui, S. W. Guo \& al. B2017-1343 (holotype: KUN).

Allocheilos rubroglandulosus W. H. Chen \& Y. M. Shui (Gesneriaceae) PhytoKeys 157: 161. 2020.

TYPE: CHINA (Yunnan*), 11/6/2017, Y. M. Shui \& al. B2017-1287 (holotype: KUN; isotype: PE).

Alocasia lihengiae C. L. Long \& Q. Fang (Araceae) Phytotaxa 436 (2): 98. 2020.

TYPE: CHINA (Yunnan*), 8/18/2018, C. L. Long 2018107 (holotype: KUN; isotype: KUN); C. L. Long 2018108-1 (paratype: KUN); C. L. Long 2018108-2 (paratype: KUN); C. L. Long 2018108-3 (paratype: KUN); C. L. Long 2018108-4 (paratype: KUN); C. L. Long 2018108-5 (paratype: KUN); C. L. Long 2018108-6 (paratype: KUN); C. L. Long 2018108-7 (paratype: KUN).

Alocasia yunqiana Z. X. Ma, Yi F. Li \& J. T. Yin (Araceae) Phytotaxa 460 (4): 278. 2020.

TYPE: CHINA (Yunnan*), 6/7/2019, Yi F. Li \& Z. X. Ma 142 (holotype: PE; isotype: A); J. T. Yin 877 (paratype: HITBC).

Alseodaphnopsis maguanensis Lang Li \& J. Li (Lauraceae) PhytoKeys 138: 31. 2020.

TYPE: CHINA (Yunnan*), 5/14/2016, Lang Li \& al. GLQ26 (holotype: HITBC); Lang Li \& al. 2016033 (paratype: HITBC).

Aristolochia wenshanensis Lei Cai, D. M. He \& Z. L. Dao (Aristolochiaceae) Taiwania 65 (1): 41. 2020.

TYPE: CHINA(Yunnan*), 3/23/2019, Lei Cai \& D. M. He CL225 (holotype: KUN; isotype: KUN,TAI); M. Q. Han HMQ1293 (paratype: IBK).

Aristolochia yachangensis B. G. Huang, Yan Liu \& Y. S. Huang (Aristolochiaceae) PhytoKeys 153: 41. 2020.

TYPE: CHINA (Guangxi*), 7/29/2019, Z. C. Lu \& al. 20190729YC4141 (holotype: IBK; isotype: GXMG, IBK); Y. J. Luo \& S. W. Li 20190421001 (paratype: IBK); Y. J. Luo \& al. YC4439 (paratype: IBK).

Aspidistra brachypetala C. R. Lin \& B. Pan (Asparagaceae) Taiwania 65 (2): 195. 2020.

TYPE: CHINA (Guangxi*), 3/28/2019, C. R. Lin 1306 (holotype: IBK; isotype: IBK).

Aspidistra dahuaensis D. X. Nong \& L. Y. Yu (Asparagaceae) Phytotaxa 472 (1): 84. 2020.

TYPE: CHINA (Guangxi*), 5/22/2019, D. X. Nong \& al. 451229190522024LY (holotype: GXMG; isotype: GXMG).

Aspidistra jiangjinensis S. R. Yi \& C. R. Lin (Asparagaceae) Phytotaxa 437 (3): 164. 2020.

TYPE: CHINA (Chongqing*), 2/13/2019, S. R. Yi YSR8101 (holotype: IBK00421261; isotype: IBK-00241262). 
杜诚, 刘军, 叶文, 廖帅, 葛斌杰, 刘冰, 马金双 (2021) 中国植物新分类群、新名称 2020 年度报告. 生物多样性, 29, 10111020. http://www.biodiversity-science.net/CN/10.17520/biods.2021122

Aspidistra longiconnectiva C. T. Lu, K. C. Chuang \& J. C. Wang (Asparagaceae) Taiwania 65 (3): 281. 2020.

TYPE: CHINA (Taiwan*), 5/15/2012, C. T. Lu 2230 (holotype: TNU; isotype: TAI).

Aspidistra xichouensis Lei Cai, Z. L. Dao \& G. W. Hu (Asparagaceae) Phytotaxa 439 (2): 145. 2020.

TYPE: CHINA (Yunnan*), 3/21/2019, Lei Cai CL221 (holotype: KUN; isotype: KUN, HIB); G. W. Hu HGW-00973 (paratype: HIB)

Aster chuanshanensis W. P. Li (Asteraceae) Annales Botanici Fennici 57 (4-6): 342. 2020.

TYPE: CHINA (Shaanxi*, Sichuan), 10/20/2015, W. P. Li LWP1510133 (holotype: HNNU; isotype: HNNU); W. P. Li LWP1608054 (paratype: HNNU); W. P. Li LWP1608055 (paratype: HNNU); W. P. Li LWP1608057 (paratype: HNNU); W. P. Li LWP1608058 (paratype: HNNU); W. P. Li LWP1608064 (paratype: HNNU); W. P. Li LWP1510130 (paratype: HNNU).

Aster huangpingensis W. P. Li \& Z. Li (Asteraceae) Phytotaxa 433 (3): 242. 2020.

TYPE: CHINA(Guizhou*), 9/13/2017, W. P. Li \& Z. Li LWPLZ1709015 (holotype: HNNU); W. P. Li \& Z. Li LWP-LZ1709008 (paratype: HNNU); W. P. Li \& Z. Li LWP-LZ1709007 (paratype: HNNU); W. P. Li \& Z. Li LWP-LZ1709006 (paratype: HNNU); Z. Li LZ1610012 (paratype: HNNU); Z. Li LZ1610013 (paratype: HNNU); Z. Li LZ1610015 (paratype: HNNU).

Aster kanoi S. W. Chung, W. J. Huang \& T. C. Hsu (Asteraceae) Taiwania 65 (3): 316. 2020.

TYPE: CHINA (Taiwan*), 8/21/2019, S. W. Chung 13946 (holotype: TAIF; isotype: TAI, HAST).

Astragalus shaerqinensis Lei Liu \& Zhi Yong Li (Fabaceae) Annales Botanici Fennici 57 (1-3): 181. 2020.

TYPE: CHINA (Nei Mongol*), 6/6/2014, Lei Liu \& Zhi Yong Li 774 (holotype: FGC; isotype: FGC).

Begonia aurora C. I Peng, Yan Liu \& W. B. Xu (Begoniaceae) Botanical Studies (Taipei) 61 (21): 3. 2020.

TYPE: CHINA (Guangxi*), 4/18/2016, C. I Peng 24765 (holotype: IBK; isotype: E, HAST-144966, K, KUN, PE); C. I Peng 23696 (paratype: HAST-144551).

Begonia depingiana Y. H. Tan \& H. B. Ding (Begoniaceae) Phytotaxa 474 (1): 82. 2020.

TYPE: CHINA (Yunnan*), 9/16/2019, D. P. Ye T0372 (holotype: HITBC-0030005; isotype: HITBC-0030006); H. B. Ding, Y. X. Gong, \& D. P. Ye T0569 (paratype: HITBC).

Begonia guangdongensis W. H. Tu, B. M. Wang \& Yu L. Li (Begoniaceae) PhytoKeys 162: 30. 2020.

TYPE: CHINA (Guangdong*), 10/26/2019, Yu L. Li \& al. 263 (holotype: CANT; isotype: IBSC).

Begonia larvata C. I Peng, Yan Liu \& W. B. Xu (Begoniaceae) Botanical Studies (Taipei) 61 (21): 7. 2020.

TYPE: CHINA (Guangxi*), 6/15/2014, C. I Peng \& al. 24372 (holotype: IBK; isotype: HAST-138465); W. B. Xu 14146 (paratype: HAST-144947).

Begonia longiornithophylla C. I Peng, W. B. Xu \& Yan Liu (Begoniaceae) Botanical Studies (Taipei) 61 (21): 10. 2020.

TYPE: CHINA (Guangxi*), 6/23/2008, C. I Peng 21518-A (holotype: IBK; isotype: E, HAST-144967, K, KUN, PE); C. I Peng 21518 (paratype: HAST-140791)

Begonia lui S. M. Ku, C. I Peng \& Yan Liu (Begoniaceae) Botanical Studies (Taipei) 61 (21): 13. 2020.

TYPE: CHINA (Guangxi*), 5/22/2007, C. I Peng 21112-A (holotype: IBK; isotype: HAST-144968); S. P. Ko 55623 (paratype: IBSC); C. I Peng 21111 (paratype: HAST-117599); C. I Peng \& al. 21112 (paratype: HAST-117600).

Begonia mengdongensis H. H. Xi (Begoniaceae) Nordic Journal of Botany 38 (e02645): 2. 2020.

TYPE: CHINA (Yunnan*), 8/6/2019, S. Y. Xiao, H. H. Xi, Y. Q. Wang \& B. Xiao KIBGX087B18 (holotype: KUN; isotype: HITBC); S. Y. Xiao, H. H.Xi, Y. Q. Wang \& B. Xiao KIBGX085B12 (paratype: KUN, HITBC).

Begonia oyuniae Taram \& N. Krishna (Begoniaceae) The Gardens' Bulletin Singapore 72 (1): 110. 2020.

TYPE: CHINA (Xizang*), 5/22/2019, M. Taram 301 (holotype: CAL; isotype: ASSAM).

Begonia puerensis W. G. Wang, X. D. Ma \& J. Y. Shen (Begoniaceae) Nordic Journal of Botany 38 (e02618): 2. 2020.

TYPE: CHINA (Yunnan*), 9/3/2019, J. Y. Shen \& X. D. Ma 1588 (holotype: HITBC; isotype: HITBC, HIB); S. S. Zhou 3024 (paratype: HITBC); S. S. Zhou 2920 (paratype: HITBC); J. Y. Shen \& X. D. Ma 1555 (paratype: HITBC); W. G. Wang \& K. H. He 1702 (paratype: HITBC).

Begonia scabrifolia C. I Peng, Yan Liu \& C. W. Lin (Begoniaceae) Botanical Studies (Taipei) 61 (21): 16. 2020. TYPE: CHINA (Guangxi*), 5/18/2009, C. I Peng 22197 (holotype: IBK; isotype: HAST-144969).

Begonia xishuangbannaensis W. G. Wang \& Li J. Jiang (Begoniaceae) Annales Botanici Fennici 57 (4-6): 249. 2020. TYPE: CHINA (Yunnan*), 11/7/2019, W. G. Wang \& K. H. He 1700 (holotype: HITBC-0031087; isotype: HITBC-0031088). 
杜诚, 刘军, 叶文, 廖帅, 葛斌杰, 刘冰, 马金双 (2021) 中国植物新分类群、新名称 2020 年度报告. 生物多样性, 29, 10111020. http://www.biodiversity-science.net/CN/10.17520/biods.2021122

Begonia zhuoyuniae C. I Peng, Yan Liu \& K. F. Chung (Begoniaceae) Botanical Studies (Taipei) 61 (21): 16. 2020.

TYPE: CHINA (Guangxi*), 12/18/2005, C. I Peng 20737-A (holotype: IBK; isotype: E, HAST-144991, K, KUN, PE); S. M. Ku 2024 (paratype: HAST-144990); C. I Peng 21061 (paratype: HAST-117106).

Berberis baiyuensis Harber (Berberidaceae) Monographs in Systematic Botany from the Missouri Botanical Garden Volume 136: 140. 2020.

TYPE: CHINA (Sichuan*), 8/21/2006, D. E. Boufford, B. Bartholomew, S. L. Kelley, R. H. Ree, H. Sun, L. L. Yue, D. C. Zhang, Y. H. Zhang \& W. D. Zhu 36945 (holotype: A; isotype: CAS, E, KUN, TI).

Berberis barkamensis Harber (Berberidaceae) Monographs in Systematic Botany from the Missouri Botanical Garden Volume 136: 140. 2020.

TYPE: CHINA (Sichuan*), 9/23/2005, C. S. Chang, H. Kim, H. I. Lim, S. Park, C. Ho, W. B. Feng, S. X. Yu \& D. M. Zheng SI0488 (holotype: SNUA-00015986; isotype: KH-B1148121, KH-B1148122, SNUA-00015987).

Berberis basumchuensis Harber (Berberidaceae) Monographs in Systematic Botany from the Missouri Botanical Garden Volume 136: 141. 2020.

TYPE: CHINA (Xizang*), 8/14/2000, D. E. Boufford, S. L. Kelley, R. H. Ree \& S. K. Wu 30026 (holotype: A-00279936; isotype: CAS1078425, HAST-115114, KUN, TI).

Berberis baxoiensis Harber (Berberidaceae) Monographs in Systematic Botany from the Missouri Botanical Garden Volume 136: 142. 2020.

TYPE: CHINA (Xizang*), 7/28/2000, D. E. Boufford, S. L. Kelley, R. H. Ree \& S. K. Wu 29770 (holotype: A-00279931; isotype: CAS1078914, HAST-115004, KUN, TI).

Berberis biguensis Harber (Berberidaceae) Monographs in Systematic Botany from the Missouri Botanical Garden Volume 136: 142. 2020.

TYPE: CHINA (Yunnan*), 8/31/2013, D. E. Boufford, J. F. Harber \& X. H. Li 43333 (holotype: PE; isotype: A, CAS, E, K, KUN, TI).

Berberis bouffordii Harber (Berberidaceae) Monographs in Systematic Botany from the Missouri Botanical Garden Volume 136: 144. 2020.

TYPE: CHINA (Qinghai*), 6/22/1995, D. E. Boufford, M. J. Donoghue, X. F. Lu \& T. S. Ying 26650 (holotype: A-00279964; isotype: CAS-26650, HAST-73898, TI-73898).

Berberis cornuta Harber (Berberidaceae) Monographs in Systematic Botany from the Missouri Botanical Garden Volume 136: 164.2020. TYPE: CHINA (Xizang*), 5/21/1936, F. Ludlow \& G. Sherriff 1626 (holotype: BM-000939688).

Berberis dahaiensis Harber (Berberidaceae) Monographs in Systematic Botany from the Missouri Botanical Garden Volume $136: 165$. 2020.

TYPE: CHINA (Yunnan*), 9/10/2013, D. E. Boufford, J. F. Harber \& X. H. Li 43529 (holotype: PE; isotype: A, BM, CAS, E, K, KUN, TI).

Berberis deqenensis Harber (Berberidaceae) Monographs in Systematic Botany from the Missouri Botanical Garden Volume $136: 171$. 2020.

TYPE: CHINA (Yunnan*), 6/2/1993, Gothenburg Exped. 683 (holotype: E-00125284; isotype: GB-0128809).

Berberis difficilis Harber (Berberidaceae) Monographs in Systematic Botany from the Missouri Botanical Garden Volume 136: 176. 2020.

TYPE: CHINA (Sichuan*), 8/26/2013, D. E. Boufford, J. F. Harber \& Q. Wang 43269 (holotype: PE; isotype: A, BM, CAS, E, K, KUN, MO, TI).

Berberis dulongjiangensis Harber (Berberidaceae) Monographs in Systematic Botany from the Missouri Botanical Garden Volume 136: 180. 2020.

TYPE: CHINA (Yunnan*), 9/14/2008, Z. L. Dao, M. Lear, Y. H. Li \& M. Wickenden 63 (holotype: PE; isotype: A, CAS, E, K, KUN, TI).

Berberis ebianensis Harber (Berberidaceae) Monographs in Systematic Botany from the Missouri Botanical Garden Volume 136: 61. 2020.

TYPE: CHINA (Sichuan*), 9/16/2010, X. J. Li 184 (holotype: TAIF-405632; isotype: TAIF-405633).

Berberis elliptifolia Harber (Berberidaceae) Monographs in Systematic Botany from the Missouri Botanical Garden Volume 136: 180. 2020.

TYPE: CHINA (Qinghai*), 6/22/1995, D. E. Boufford, M. J. Donoghue, X. F. Lu \& T. S. Ying 26652 (holotype: A-00279960; isotype: MO-4920164). 
杜诚, 刘军, 叶文, 廖帅, 葛斌杰, 刘冰, 马金双 (2021) 中国植物新分类群、新名称 2020 年度报告. 生物多样性, 29, 10111020. http://www.biodiversity-science.net/CN/10.17520/biods.2021122

Berberis emeishanensis Harber (Berberidaceae) Monographs in Systematic Botany from the Missouri Botanical Garden Volume 136 : 181. 2020.

TYPE: CHINA (Sichuan*), 6/1/1904, E. H. Wilson (Veitch) 4726A (holotype: A-00279867; isotype: A-00279868, IBSC-0000591, K, P02313289).

Berberis epedicellata Harber (Berberidaceae) Monographs in Systematic Botany from the Missouri Botanical Garden Volume 136: 182. 2020.

TYPE: CHINA (Sichuan*), 9/20/1991, J. Simmons, C. Erskine, C. Howick \& W. McNamara 538 (holotype: K).

Berberis exigua Harber (Berberidaceae) Monographs in Systematic Botany from the Missouri Botanical Garden Volume 136: 184.2020. TYPE: CHINA (Yunnan*), 9/2/2005, S. \& D. Rankin 4219 (holotype: PE; isotype: A, BM, KUN, MO, P, TI).

Berberis gaoshanensis Harber (Berberidaceae) Monographs in Systematic Botany from the Missouri Botanical Garden Volume 136: 188. 2020.

TYPE: CHINA (Sichuan*), 8/27/2013, D. E. Boufford, J. F. Harber \& Q. Wang 43280 (holotype: PE; isotype: A, CAS, E, K, KUN, TI).

Berberis gongshanensis Harber (Berberidaceae) Monographs in Systematic Botany from the Missouri Botanical Garden Volume 136: 192. 2020.

TYPE: CHINA (Yunnan*), 7/17/2000, H. Li, B. Bartholomew, P. Thomas, P. W. Fritsch, Z. L. Dao, Z. L. Wang \& R. Li 12776 (holotype: E-00238635; isotype: CAS-00120137, GH-00280003, HAST-89937, KUN-1410011, MO-5754350).

Berberis gyaitangensis Harber (Berberidaceae) Monographs in Systematic Botany from the Missouri Botanical Garden Volume 136: 192. 2020.

TYPE: CHINA (Yunnan*), 6/15/2016, P. Brownless 1049 (holotype: E-00831857; isotype: A).

Berberis heishuiensis Harber (Berberidaceae) Monographs in Systematic Botany from the Missouri Botanical Garden Volume 136: 194. 2020.

TYPE: CHINA (Sichuan*), 5/8/2014, D. E. Boufford, S. Cristoph, C. Davidson, Y. D. Gao \& Q. Y. Xiang 43610 (holotype: PE; isotype: A, CDBI, E, MO, TI).

Berberis hubianensis Harber (Berberidaceae) Monographs in Systematic Botany from the Missouri Botanical Garden Volume 136: 199. 2020.

TYPE: CHINA (Yunnan*), 8/31/2013, D. E. Boufford, J. F. Harber \& X. H. Li 43336 (holotype: PE; isotype: A, E, KUN).

Berberis kangwuensis Harber (Berberidaceae) Monographs in Systematic Botany from the Missouri Botanical Garden Volume 136: 208. 2020.

TYPE: CHINA (Sichuan*), 9/7/2013, D. E. Boufford, J. F. Harber \& X. H. Li 43494 (holotype: PE; isotype: A, BM, CAS, E, HAST, IBSC, K, KUN, MO, PE, TI).

Berberis lhunzensis Harber (Berberidaceae) Monographs in Systematic Botany from the Missouri Botanical Garden Volume 136: 213. 2020.

TYPE: CHINA (Xizang*), 5/15/2013, V. Gallavan s.n. (holotype: PE; isotype: A, E).

Berberis lhunzhubensis Harber (Berberidaceae) Monographs in Systematic Botany from the Missouri Botanical Garden Volume 136: 214. 2020.

TYPE: CHINA (Xizang*), 7/12/1944, F. Ludlow \& G. Sherriff 9974 (holotype: BM-000939687; isotype: E-00351618).

Berberis lixianensis Harber (Berberidaceae) Monographs in Systematic Botany from the Missouri Botanical Garden Volume 136: 215. 2020.

TYPE: CHINA (Sichuan*), 5/9/2014, D. E. Boufford, S. Cristoph, C. Davidson, Y. D. Gao \& Q. Y. Xiang 43624 (holotype: PE; isotype: A, CDBI, E, MO, TI).

Berberis longipedicellata Harber (Berberidaceae) Monographs in Systematic Botany from the Missouri Botanical Garden Volume 136 215. 2020.

TYPE: CHINA (Sichuan*), 7/28/2007, D. E. Boufford, K. Fujikawa, S. L. Kelley, R. H. Ree, B. Xu, D. C. Zhang, J. W. Zhang, T. C. Zhang \& W. D. Zhu 38505 (holotype: A; isotype: CAS, E, KUN, MO, TI).

Berberis mabiluoensis Harber (Berberidaceae) Monographs in Systematic Botany from the Missouri Botanical Garden Volume 136: 217. 2020.

TYPE: CHINA (Yunnan*), 9/25/2008, Z. L. Dao, M. Lear, Y. H. Li \& M. Wickenden 304 (holotype: PE; isotype: A, BM, CAS, E, IBSC, K, KUN, MO, TI, WSY).

Berberis markamensis Harber (Berberidaceae) Monographs in Systematic Botany from the Missouri Botanical Garden Volume 136: 218. 2020. 
杜诚, 刘军, 叶文, 廖帅, 葛斌杰, 刘冰, 马金双 (2021) 中国植物新分类群、新名称 2020 年度报告. 生物多样性, 29, 10111020. http://www.biodiversity-science.net/CN/10.17520/biods.2021122

TYPE: CHINA (Xizang*), 6/15/1976, Qinghai-Xizang Exped. 11882 (holotype: KUN-0178848; isotype: KUN-0178849, PE-01037688, PE-01037829, HNWP).

Berberis monticola Harber (Berberidaceae) Monographs in Systematic Botany from the Missouri Botanical Garden Volume 136: 223. 2020.

TYPE: CHINA (Yunnan*), 8/29/2013, D. E. Boufford, J. F. Harber \& Q. Wang 43304 (holotype: PE; isotype: A, E, KUN).

Berberis nanifolia Harber (Berberidaceae) Monographs in Systematic Botany from the Missouri Botanical Garden Volume 136: 227. 2020.

TYPE: CHINA (Yunnan*), 9/4/2013, D. E. Boufford, J. F. Harber \& X. H. Li 43437 (holotype: PE-01892852; isotype: A-00914432, BM-001190939, CAS, E-00833562, E-00833563, HAST, IBSC, K, KUN, MO-1204038, TI).

Berberis ngawaica Harber (Berberidaceae) Monographs in Systematic Botany from the Missouri Botanical Garden Volume 136: 228. 2020.

TYPE: CHINA (Sichuan*), 5/9/2014, D. E. Boufford, S. Cristoph, C. Davidson, Y. D. Gao \& Q. Y. Xiang 43640 (holotype: PE; isotype: A, CDBI, E, MO, TI).

Berberis ninglangensis Harber (Berberidaceae) Monographs in Systematic Botany from the Missouri Botanical Garden Volume 136: 229. 2020.

TYPE: CHINA (Yunnan*), 7/1/1922, G. Forrest 22051 (holotype: E-00570041; isotype: A-00105609, CAL-0000027192, K, US009465992).

Berberis nujiangensis Harber (Berberidaceae) Monographs in Systematic Botany from the Missouri Botanical Garden Volume 136: 92. 2020.

TYPE: CHINA (Xizang*), 9/1/1935, C. W. Wang 66201 (holotype: A-00038691; isotype: KUN-0177061, NAS-00314333, PE-01031007, PE-01031008, PE-01031009, WUK-0047683)

Berberis nyingchiensis Harber (Berberidaceae) Monographs in Systematic Botany from the Missouri Botanical Garden Volume 136: 229. 2020.

TYPE: CHINA (Xizang*), 5/21/1947, F. Ludlow, G. Sherriff \& H. H. Elliot 13731 (holotype: BM-000794117; isotype: A-00279577, E00258233).

Berberis pluvisylvatica Harber (Berberidaceae) Monographs in Systematic Botany from the Missouri Botanical Garden Volume 136: 234. 2020.

TYPE: CHINA (Xizang*), 5/30/1947, F. Ludlow, G. Sherriff \& H. H. Elliot 15077 (holotype: BM-000939719).

Berberis polybotrys Harber (Berberidaceae) Monographs in Systematic Botany from the Missouri Botanical Garden Volume 136: 235. 2020.

TYPE: CHINA (Yunnan*), 10/11/1994, P. Brownless 1048 (holotype: E-00831859; isotype: A, PE).

Berberis pratensis Harber (Berberidaceae) Monographs in Systematic Botany from the Missouri Botanical Garden Volume $136: 238$. 2020.

TYPE: CHINA (Sichuan*), 8/28/2013, D. E. Boufford, J. F. Harber \& Q. Wang 43293 (holotype: PE; isotype: A, E, KUN).

Berberis pruinosifolia Harber (Berberidaceae) Monographs in Systematic Botany from the Missouri Botanical Garden Volume 136: 240. 2020.

TYPE: CHINA (Sichuan*), 6/1/1929, J. F. Rock 17775 (holotype: E-00392889; isotype: A-00279469, A-00279472, IBSC-0091670, US00945922).

Berberis purpureocaulis Harber (Berberidaceae) Monographs in Systematic Botany from the Missouri Botanical Garden Volume 136: 243. 2020.

TYPE: CHINA (Sichuan*), 6/1/1929, J. F. Rock 17957 (holotype: A-00279718; isotype: K, P-02682339, US-00945962, US-00946000).

Berberis qamdoensis Harber (Berberidaceae) Monographs in Systematic Botany from the Missouri Botanical Garden Volume 136: 243. 2020.

TYPE: CHINA (Xizang*), 8/12/2004, D. E. Boufford, J. H. Chen, S. L. Kelley, J. Li, R. H. Ree, H. Sun, J. P. Yue \& Y. H. Zhan 32224 (holotype: A-00279934; isotype: KUN).

Berberis qinghaiensis Harber (Berberidaceae) Monographs in Systematic Botany from the Missouri Botanical Garden Volume 136: 245. 2020.

TYPE: CHINA (Qinghai*), 8/9/2007, D. E. Boufford, K. Fujikawa, S. L. Kelley, R. H. Ree, B. Xu, D. C. Zhang, J. W. Zhang, T. C. Zhang, W. D. Zhu 39340 (holotype: A; isotype: CAS, E, F, KUN, PE, TI).

Berberis saltuensis Harber (Berberidaceae) Monographs in Systematic Botany from the Missouri Botanical Garden Volume 136: 248. 2020. 
杜诚, 刘军, 叶文, 廖帅, 葛斌杰, 刘冰, 马金双 (2021) 中国植物新分类群、新名称 2020 年度报告. 生物多样性, 29, 10111020. http://www.biodiversity-science.net/CN/10.17520/biods.2021122

TYPE: CHINA (Sichuan*), 9/7/2013, D. E. Boufford, J. F. Harber \& X. H. Li 43496 (holotype: PE; isotype: A, BM, CAS, E, K, KUN, MO, TI).

Berberis saxatilis Harber (Berberidaceae) Monographs in Systematic Botany from the Missouri Botanical Garden Volume 136: 249. 2020.

TYPE: CHINA (Yunnan*), 7/25/2007, J. Wen, Z. Nie, R. J. Soreng, K. B. Ramkin, L. L. Yue, M. Wang \& X. K. Yue: 1282 (holotype: US00971881; isotype: KUN).

Berberis scrithalis Harber (Berberidaceae) Monographs in Systematic Botany from the Missouri Botanical Garden Volume 136: 249. 2020.

TYPE: CHINA (Yunnan*), 10/7/1994, Alpine Garden Society China Exped. (ACE) 1847 (holotype: E-00039648; isotype: LIV2005.15.1184, WSY-0057777).

Berberis taoensis Harber (Berberidaceae) Monographs in Systematic Botany from the Missouri Botanical Garden Volume 136: 257. 2020. TYPE: CHINA (Gansu*), 6/1/1925, J. F. Rock 12443 (holotype: A-00279330; isotype: E-00623028, P-02313649).

Berberis tengchongensis Harber (Berberidaceae) Monographs in Systematic Botany from the Missouri Botanical Garden Volume 136: 259. 2020.

TYPE: CHINA (Yunnan*), 5/27/2006, Gaoligong Biodiversity Survey 200630682 (holotype: CAS-00120135; isotype: GH-00285287, HAST-124191, KUN-1409997, MO-6057223).

Berberis tengii Harber (Berberidaceae) Monographs in Systematic Botany from the Missouri Botanical Garden Volume 136: 111. 2020. TYPE: CHINA (Guizhou*), 4/16/1936, S. W. Teng 90155 (holotype: A-00280084; isotype: IBK-00012848, IBSC-0092096, L-0831786, MO-2205171).

Berberis tenuispina Harber (Berberidaceae) Monographs in Systematic Botany from the Missouri Botanical Garden Volume $136: 260$. 2020.

TYPE: CHINA (Yunnan*), 7/27/2007, Tibet-MacArthur 1373 (holotype: US-00972148; isotype: KUN).

Berberis tianchiensis Harber (Berberidaceae) Monographs in Systematic Botany from the Missouri Botanical Garden Volume 136: 262. 2020.

TYPE: CHINA (Yunnan*), 8/31/2013, D. E. Boufford, J. F. Harber \& X. H. Li 43334 (holotype: PE; isotype: A, CAS, E, K, KUN).

Berberis wenchuanensis Harber (Berberidaceae) Monographs in Systematic Botany from the Missouri Botanical Garden Volume 136 : 273. 2020.

TYPE: CHINA (Sichuan*), 6/20/1908, E. H. Wilson 2857 (holotype: A-00279860; isotype: BM-000810083, E-00612583l, K, LE, US00945943).

Berberis wui Harber (Berberidaceae) Monographs in Systematic Botany from the Missouri Botanical Garden Volume 136: 117. 2020.

TYPE: CHINA (Yunnan*), 10/25/1994, P. Brownless 183 (holotype: E-00615601).

Berberis xiangchengensis Harber (Berberidaceae) Monographs in Systematic Botany from the Missouri Botanical Garden Volume 136: 280. 2020.

TYPE: CHINA (Sichuan*), 8/24/2013, D. E. Boufford, J. F. Harber \& Q. Wang 43202 (holotype: PE; isotype: A, CAS, E, K, KUN, TI).

Berberis xiaozhongdianensis Harber (Berberidaceae) Monographs in Systematic Botany from the Missouri Botanical Garden Volume 136: 280. 2020.

TYPE: CHINA (Yunnan*), 9/4/2013, D. E. Boufford, J. F. Harber \& X. H. Li 43440 (holotype: PE; isotype: A, E, KUN)

Berberis yaanica Harber (Berberidaceae) Monographs in Systematic Botany from the Missouri Botanical Garden Volume 136: 281. 2020. TYPE: CHINA (Sichuan*), 5/14/2014, D. E. Boufford, S. Cristoph, C. Davidson \& Y. D. Gao 43738 (holotype: CDBI; isotype: A, CAS, E, MO,P, PE, SRP, TI).

Berberis yalongensis Harber (Berberidaceae) Monographs in Systematic Botany from the Missouri Botanical Garden Volume 136: 281. 2020.

TYPE: CHINA (Sichuan*), 8/2/2005, D. E. Boufford, J. H. Chen, K. Fujikawa, S. L. Kelley, R. H. Ree, H. Sun, J. P. Yue, D. C. Zhang \& Y. H. Zhang 33983 (holotype: A-00279952; isotype: CAS-1078422, KUN, TI).

Berberis yanyuanensis Harber (Berberidaceae) Monographs in Systematic Botany from the Missouri Botanical Garden Volume 136: 282. 2020.

TYPE: CHINA (Sichuan*), 5/12/1914, C. K. Schneider 1196 (holotype: A-00279733; isotype: K).

Berberis yarigongensis Harber (Berberidaceae) Monographs in Systematic Botany from the Missouri Botanical Garden Volume 136: 282. 2020. 
杜诚, 刘军, 叶文, 廖帅, 葛斌杰, 刘冰, 马金双 (2021) 中国植物新分类群、新名称 2020 年度报告. 生物多样性, 29, 10111020. http://www.biodiversity-science.net/CN/10.17520/biods.2021122

TYPE: CHINA (Sichuan*), 7/1/1904, J. A. Soulié 3057 (holotype: P-02682030; isotype: K-001273511, MO-6318150, P-02682028, PE01969512, PE-01969513, SING-0155672).

Berberis yingii Harber (Berberidaceae) Monographs in Systematic Botany from the Missouri Botanical Garden Volume 136: 283.2020. TYPE: CHINA (Sichuan*), 10/7/1994, P. Brownless 492 (holotype: E-00705631).

Berberis yulongshanensis Harber (Berberidaceae) Monographs in Systematic Botany from the Missouri Botanical Garden Volume 136: 284. 2020.

TYPE: CHINA (Yunnan*), 6/5/1985, Kunming-Edinburgh Yulong-Shan Exped. (KEY) 538 (holotype: E-00612570; isolectotype: KUN0176571).

Berberis yushuensis Harber (Berberidaceae) Monographs in Systematic Botany from the Missouri Botanical Garden Volume 136: 286. 2020.

TYPE: CHINA (Qinghai*), 8/18/1996, T. N. Ho, B. Bartholomew, M. F. Watson \& M. G. Gilbert 2028 (holotype: GH-00279965; isolectotype: BM-001010958, CAS-935986, E-00065379, HNWP-0286562, MO-5329351, PE-01840129).

Berberis zhaoi Harber (Berberidaceae) Monographs in Systematic Botany from the Missouri Botanical Garden Volume 136: 287.2020. TYPE: CHINA (Yunnan*), 5/1/1910, G. Forrest 5553 (holotype: E-00612485; isolectotype: BM-000810140, IBSC-0091908, K001273250, N-093057077, P-02313228, PE-01033864, PE-01033865, UC-224593).

Berberis zhenxiongensis Harber (Berberidaceae) Monographs in Systematic Botany from the Missouri Botanical Garden Volume 136 : 122. 2020.

TYPE: CHINA (Yunnan*), 6/8/1980, S. Y. Bao 106 (holotype: KUN-0175742; isolectotype: KUN-0175743).

Berberis zhongdianensis Harber (Berberidaceae) Monographs in Systematic Botany from the Missouri Botanical Garden Volume 136: 288. 2020.

TYPE: CHINA (Yunnan*), 6/21/1994, Alpine Garden Society China Exped. (ACE) 507 (holotype: E-00045830; isolectotype: K).

Blepharostoma neglecta Vilnet \& Bakalin (Blepharostomataceae) Plants 9 (1423): 22. 2020.

TYPE: CHINA (Sichuan, Yunnan), RUSSIA(T)RUSSIA(T) 10/1/2016, V.A. Bakalin 1 October 2016 S-48-18-16 (holotype: VBGI; isotype: $\mathrm{KPABG}$ ).

Blumea hunanensis L. D. Duan \& Yun Lin (Asteraceae) Kew Bulletin 75 (9): 1. 2020.

TYPE: CHINA (Hunan*), 1/21/2015, L. D. Duan \& Z. Lu 5813 (holotype: PE-02232681; isotype: Herbarium, Hunan Food and Drug Vocational College, Hunan, China, Herbarium, Shaoyang University, Hunan, China, BM, E, GZAC, IBK, IBSC, K, KUN, MO, PE02232682).

Boeica arunachalensis D. Borah, R. Kr. Singh, Taram \& A. P. Das (Gesneriaceae) Indian Forester 146 (9): 871.2020.

TYPE: CHINA (Xizang*), 9/12/2018, D. Borah \& M. Taram 93 (holotype: ASSAM; isotype: ARUN, ASSAM).

Bredia hispida J. H. Dai \& Ying Liu (Melastomataceae) PhytoKeys 152: 9. 2020.

TYPE: CHINA (Sichuan*), 9/1/2019, Ying Liu 764 (holotype: PE; isotype: A, SYS)

Bredia reniformis C. M. He, Y. H. Tong \& S. Jin Zeng (Melastomataceae) Nordic Journal of Botany 38 (e02525): 2. 2020.

TYPE: CHINA (Guangxi*), 10/10/2017, Y. H. Tong TYH-1376 (holotype: IBSC; isotype: HIB, IBSC, IBK, KUN, PE); Y. H. Tong TYH417 (paratype: IBSC); W. B Xu, Y. S. Huang \& S. W. Li 13176 (paratype: IBK, CANT)

Bulbophyllum gedangense Y. Luo, J. P. Deng \& Jian W. Li (Orchidaceae) Phytotaxa 453 (2): 146. 2020.

TYPE: CHINA (Xizang*), 8/23/2019, Y. Luo \& al. 1239 (holotype: HITBC-0030001; isotype: HITBC-0030002, HITBC-0030003); Jin \& al. 19192 (paratype: HITBC, PE, XZ)

Bulbophyllum lingii M. Z. Huang, G. S. Yang \& J. M. Yin (Orchidaceae) Phytotaxa 452 (2): 185. 2020.

TYPE: CHINA (Hainan*), 5/29/2013, M. Z. Huang 13052901 (holotype: ATCH); M. Z. Huang 15060501 (paratype: ATCH)

Bulbophyllum linzhiense Liang Ma \& S. P. Chen (Orchidaceae) Phytotaxa 429 (4): 287. 2020.

TYPE: CHINA (Xizang*), 10/2/2017, Liang Ma 20171042xz (holotype: FAFU).

Burmannia decurrens Xiao Juan Li \& D. X. Zhang (Burmanniaceae) Nordic Journal of Botany 38 (e02718): 3. 2020.

TYPE: CHINA (Guangdong*), 9/9/2019, Xiao Juan Li, K. Zhang,G. Zeng \& M. S. Wu 01210 (holotype: IBSC; isotype: HIB, IBSC, KUN); T. Zeng \& Q. B. Zeng ZengT0011 (paratype: IBSC); T. Zeng \& Q. B. Zeng ZengT0013 (paratype: IBSC, KUN)

Calamagrostis hongii Paszko \& Bing Liu (Poaceae) PhytoKeys 166: 43. 2020.

TYPE: CHINA (Chongqing, Guizhou, Sichuan, Xizang, Yunnan*), 8/18/2008, Y. M. Shui \& al. 64471 (holotype: PE-2308966; isotype: PE-2058824, PE-2070270). 
杜诚, 刘军, 叶文, 廖帅, 葛斌杰, 刘冰, 马金双 (2021) 中国植物新分类群、新名称 2020 年度报告. 生物多样性, 29, 10111020. http://www.biodiversity-science.net/CN/10.17520/biods.2021122

Calanthe sieboldopsis Bo Y. Yang \& Bo Li (Orchidaceae) PhytoKeys 145: 39. 2020.

TYPE: CHINA (Jiangxi*), 4/19/2017, Bo Y. Yang 095 (holotype: CSH; isotype: JXU).

Calanthe tsiana Y. Q. Chen, J. W. Zhai \& S. R. Lan (Orchidaceae) Phytotaxa 428 (1): 70. 2020.

TYPE: CHINA (Yunnan*), 2/15/2019, Y. Q. Chen 0628 (holotype: FJFC).

Camellia hainanica Y. L. Zhao \& Z. G. Xu (Theaceae) Pakistan Journal of Botany 52 (3): 1028. 2020.

TYPE: CHINA (Hainan*), 8/26/2017, Y. L. Zhao \& Z. G. Xu 170801 (holotype: SYS; isotype: IBSC); Y. L. Zhao \& X. G. Shi 161131 (paratype: SYS); Y. C. Tang \& L. Wu 161021 (paratype: CSFI); L. Wu \& W. Zhang 161030 (paratype: CSFI).

Camellia rostrata S. X. Yang \& S. F. Chai (Theaceae) Phytotaxa 459 (1): 62.2020.

TYPE: CHINA (Guangxi*), 9/26/2019, S. X. Yang 6181 (holotype: KUN-1482804; isotype: KUN, PE, K, P).

Camellia zhaiana S. X. Yang (Theaceae) Phytotaxa 460 (3): 225. 2020.

TYPE: CHINA (Guangxi*), 1/16/2020, S. X. Yang \& P. M. Ye 6324 (holotype: KUN-1482785; isotype: K, KUN, PE).

Carex ledongensis H. B. Yang \& G. D. Liu (Cyperaceae) Phytotaxa 461 (2): 72.2020.

TYPE: CHINA (Hainan*), 4/25/2019, H. B. Yang 201904254614 (holotype: IBSC; isotype: ATCH).

Carex pingleensis Z. C. Lu, Y. F. Lu \& X. F. Jin (Cyperaceae) Taiwania 65 (3): 391. 2020.

TYPE: CHINA (Guangxi*), 5/20/2019, Y. L. Su \& J. Q. Huang LZC199 (holotype: IBK-00421264; isotype: IBK-00421266, HTC).

Carpinus gigabracteatus Z. Qiang Lu (Betulaceae) PhytoKeys 145: 50. 2020.

TYPE: CHINA (Yunnan*), 9/23/2019, Z. Qiang Lu 2019GY0801 (holotype: HITBC; isotype: HITBC, LZU).

Caryopteris alternifolia Y. S. Chen \& C. L. Xiang (Lamiaceae) Phytotaxa 461 (1): 64. 2020.

TYPE: CHINA (Guangdong*), 12/1/2019, Y. S. Chen \& Z. C. Jin 19065 (holotype: IBSC; isotype: KUN).

Causonis wentsiana Z. H. Chen, F. Chen \& X. F. Jin (Vitaceae) Phytotaxa 475 (4): 257. 2020.

TYPE: CHINA (Zhejiang*), 7/10/2017, Z. H. Chen, W. Y. Xie \& L. X. Zheng WC17061004 (holotype: ZM; isotype: HTC, PE); Z. H. Chen \& F. H. Chi s.n. (paratype: ZJFC); Z. H. Chen, F. Chen \& L. X. Zheng WC17080303 (paratype: ZM); Z. H. Chen, J. S. Wang \& X. Liu WC18062201 (paratype: HTC); Z. H. Chen, J. S. Wang \& X. Liu WC18062202 (paratype: HTC); C. Z. Zheng \& C. F. Zhang 7501 (paratype: HTC).

Causonis wentsiana Z. H. Chen, Y. F. Lu \& X. F. Jin (Vitaceae) Phytotaxa 475 (4): 261. 2020.

TYPE: CHINA (Zhejiang*), 8/1/2017, Z. H. Chen, F. Chen \& W. Y. Xie JN17080104A (holotype: ZM; isotype: HTC, PE); Z. H. Chen, W. Y. Xie \& J. S. Wang JS17061202 (paratype: ZM); Z. H. Chen JS18050702 (paratype: ZM); Z. H. Chen JS18050703 (paratype: ZM); Z. H. Chen \& al. 18072901 (paratype: HTC); Z. H. Chen, J. S. Wang \& X. Liu TS18062201 (paratype: HTC); Z. H. Chen, J. S. Wang \& X. Liu TS18062202 (paratype: HTC). Z. H. Chen, F. Chen \& G. Y. Li CN-07 (paratype: ZJFC); Z. H. Chen, F. Chen \& G. Y. Li CN08 (paratype: ZJFC); Z. H. Chen, F. Chen \& G. Y. Li CN-011 (paratype: ZJFC); Z. H. Chen, J. S. Wang \& J. Lin JN18062003 (paratype: HTC); Z. H. Chen, J. S. Wang \& J. Lin JN18062004 (paratype: HTC); Z. H. Chen, J. Lin \& X. F. Xu JN18052005 (paratype: ZM); Z. H. Chen, W. Y. Xie \& S. L. Zhen QY17061101 (paratype: ZM).

Chamaelirium shimentaiense Y. H. Tong, C. M. He \& Y. Q. Li (Melanthiaceae) Nordic Journal of Botany 38 (e02634): 2. 2020. TYPE: CHINA (Guangdong*), 4/22/2017, Y. Q. Li TYH-983 (holotyp: IBSC; isotype: KUN); Y. Q. Li TYH-983 (paratype: IBSC).

Cheirostylis barbata Q. Liu \& X. F. Wu (Orchidaceae) Phytotaxa 436 (3): 290. 2020.

TYPE: CHINA (Yunnan*), 3/21/2019, Q. Liu 690 (holotype: YFS); Q. Liu 690 (paratype: HFTC)

Chrysanthemum bizarre C. Z. Shen (Asteraceae) Phytotaxa 442 (3): 220. 2020.

TYPE: CHINA (Hunan*), 9/30/2019, L. Wu 19093025 (holotype: CSFI; isotype: PE); J. J. Zhou 14100601(paratype: CSFI-027294, CSFI-027295, CSFI-027296, CSFI-027297).

Chrysosplenium zhouzhiense Hong Liu bis (Saxifragaceae) PhytoKeys 159: 129. 2020.

TYPE: CHINA (Shaanxi*), 5/10/2020, Hong Liu, D. Q. Lan \& W. Huang HSN13356 (holotype: HSN; isotype: HSN, IBK).

Cleisostoma hainanense M. Z. Huang, D. K. Liu \& G. S. Yang (Orchidaceae) Phytotaxa 428 (3): 270. 2020.

TYPE: CHINA (Hainan*), 3/16/2011, M. Z. Huang 13070201 (holotype: ATCH).

Crepidomanes shenzhenense Hui Wang bis \& X. Yun Wang (Hymenophyllaceae) Phytotaxa 440 (2): 102. 2020.

TYPE: CHINA (Guangdong*), 1/7/2017, G. H. Zhao, X. Yun Wang \& T. Xu 1701006 (holotype: SZG-00109026; isotype: KUN, MO, PE, SZG-00109002, SZG-00109027, SZG-00109028). 
杜诚, 刘军, 叶文, 廖帅, 葛斌杰, 刘冰, 马金双 (2021) 中国植物新分类群、新名称 2020 年度报告. 生物多样性, 29, 10111020. http://www.biodiversity-science.net/CN/10.17520/biods.2021122

Cupressus $\times$ wangii J. Hoch, Maerki \& Rushforth (Cupressaceae) Bulletin of the Cupressus Conservation Project 9 (2): 20.2020.

TYPE: CHINA, 1/3/2003, Silba B-330 (holotype: NY-00658653).

Cupressus gansuensis Maerki \& J. Hoch (Cupressaceae) Bulletin of the Cupressus Conservation Project 9 (1): 4.2020.

TYPE: CHINA (Gansu*), 7/11/1951, T. P. Wang 14286 (holotype: PE; isotype: HIB, WUK); T. P. Wang 14304 (paratype: PE, WUK); Rock 12073 (paratype: E, P, K); Meyer 1981 (paratype: K, P, NY).

Cyclobalanopsis reclinatocaulis M. M. Lin (Fagaceae) Bulletin of Botanical Research 40 (1): 11. 2020.

TYPE: CHINA (Fujian*), 10/16/2013, Q. W. Lin \& al. 01-06 (holotype: AU).

Cymbidium $\times$ malipoense L. J. Chen, Hong B. Zhou \& Z. J. Liu (Orchidaceae) Plant Science Journal 38 (2): 182.2020.

TYPE: CHINA (Yunnan*), 12/22/2011, Hong B. Zhou 0001 (holotype: NOCC).

Cymbidium biflorens D. Y. Zhang, S. R. Lan \& Z. J. Liu (Orchidaceae) Phytotaxa 428 (3): 274. 2020.

TYPE: CHINA (Yunnan*), 8/1/2019, D. Y. Zhang F001 (holotype: FJFC).

Cymbidium brevifolium Zhuang Zhou, S. R. Lan \& Z. J. Liu (Orchidaceae) Phytotaxa 464 (3): 240. 2020.

TYPE: CHINA (Hubei*), 7/25/2020, Zhuang Zhou F001 (holotype: FJFC).

Cymbidium codonanthum Y. T. Jiang, Liang Ma \& S. P. Chen (Orchidaceae) Phytotaxa 453 (3): 281. 2020.

TYPE: CHINA (Yunnan*), 11/6/2018, Liang Ma F007 (holotype: FJFC).

Cyrtomium semifertile Jian X. Li \& Xiao J. Li (Dryopteridaceae) Bangladesh Journal of Botany 49 (3): 703. 2020.

TYPE: CHINA (Shandong*), 8/27/2012, Jian X. Li 816 (holotype: PE; isotype: SDCM).

Daphniphyllum peltatum Yan Liu \& T. Meng (Daphniphyllaceae) Taiwania 65 (2): 232. 2020.

TYPE: CHINA (Guangxi*), 6/24/2012, Y. S. Huang \& W. B. Xu $20120624 Y 1592$ (holotype: IBK; isotype: IBK, GXMG); Y. S. Huang \& D. X. Nong Y0669 (paratype: IBK); Y. S. Huang Y0738 (paratype: IBK); Y. S. Huang \& Y. B. Liao Y1052 (paratype: IBK); Y. S. Huang Y1232 (paratype: TAI); X. Y. Huang \& al. $451025130316001 L Y$ (paratype: IBK, GXMG).

Deinostigma fasciculatum W. H. Chen \& Y. M. Shui (Gesneriaceae) PhytoKeys 157: 201. 2020.

TYPE: CHINA (Yunnan*), 8/11/2018, Y. M. Shui \& S. W. Guo B2018-493 (holotype: KUN).

Delphinium adenopodum W. T. Wang \& Z. Z. Yang (Ranunculaceae) Bulletin of Botanical Research 40 (6): 801.2020.

TYPE: CHINA (Xinjiang*), 8/3/2019, Z. Z. Yang 7659 (holotype: PE).

Delphinium gongliuense W. T. Wang \& Z. Z. Yang (Ranunculaceae) Bulletin of Botanical Research 40 (6): 804.2020.

TYPE: CHINA (Xinjiang*), 7/28/2018, Z. Z. Yang 0817 (holotype: PE-02331415; isoteype: PE).

Dendrobium jinghuanum B. Q. Zheng \& Yan Wang (Orchidaceae) Phytotaxa 428 (1): 40.2020.

TYPE: CHINA (Yunnan*), 6/11/2018, B. Q. Zheng 001 (holotype: CAF).

Dendrobium yongjiaense Zhuang Zhou \& S. R. Lan (Orchidaceae) Phytotaxa 441 (2): 209. 2020.

TYPE: CHINA (Zhejiang*), 12/6/2019, Zhuang Zhou 2019120601 (holotype: FAFC).

Didymocarpus lobulatus F. Wen, Xin Hong \& W. Y. Xie (Gesneriaceae) PhytoKeys 157: 147. 2020.

TYPE: CHINA (Zhejiang*), 5/23/2014, W. Y. Xie \& J. J. Zhou 140523-01 (holotype: IBK; isotype: AHU).

Didymocarpus longicalyx G. W. Hu \& Q. F. Wang (Gesneriaceae) Phytotaxa 475 (1): 62.2020.

TYPE: CHINA (Yunnan*), 7/27/2019, G. W. Hu, S. Peng \& J. J. Wang HGW-1175 (holotype: HIB-0283879; isotype: HIB-0283800, HIB-0283801, IBSC); G. W. Hu \& al. HGW-2024 (paratype: HIB).

Didymocarpus sinoindicus N. S. Prasanna, Lei Cai \& V. Gowda (Gesneriaceae) Rheedea 30 (1): 136. 2020.

TYPE: CHINA (Yunnan), INDIA*, 8/29/2018, Preeti Saryan VG2018NL3679 (holotype: BHPL; isotype: ASSAM).

Dimetia brevipetiolata R. J. Wang (Rubiaceae) Phytotaxa 428 (1): 45.2020.

TYPE: CHINA (Guangxi*), 4/2/2013, R. J. Wang \& S. J. Deng 2304 (holotype: IBSC-0847225; isotypes: IBSC); R. J. Wang 2083 (paratype: IBSC); R. J. Wang \& S. J. Deng 2305 (paratype: IBSC); G. B. Jiang, X. X. Zhou \& M. D. Yuan 1257 (paratype: IBSC).

Diplophyllum purpurascens Bakalin \& Vilnet (Scapaniaceae) Phytotaxa 447 (2): 120. 2020.

TYPE: CHINA (Yunnan*), 10/11/2018, V.A. Bakalin \& W.Z. Ma C-73-29-18 (holotype: VBGI; isotype: KPABG, KUN).

Encalypta papillosa C. Feng, J. Kou \& B. Niu (Encalyptaceae) Journal of Bryology 42 (4): 327. 2020. 
杜诚, 刘军, 叶文, 廖帅, 葛斌杰, 刘冰, 马金双 (2021) 中国植物新分类群、新名称 2020 年度报告. 生物多样性, 29, 10111020. http://www.biodiversity-science.net/CN/10.17520/biods.2021122

TYPE: CHINA (Xizang*), 8/25/2018, B. Niu 20180825003 (holotype: NMAC; isotype: NY); B. Niu 20180826001 (paratype: NMAC); B. Niu 20180824002 (paratype: NMAC)

Entosthodon elimbatus W. Z. Ma, Shevock \& S. He (Funariaceae) Phytotaxa 451 (2): 108. 2020.

TYPE: CHINA (Yunnan*), 9/18/2013, W.Z. Ma \&

Shevock 13-5097 (holotype: KUN; isotypes: CAS, CONN, KRAM, MO).

Eriobotrya laoshanica W. B. Liao, Q. Fan \& Su F. Chen (Rosaceae) PhytoKeys 146: 64. 2020.

TYPE: CHINA (Yunnan*), 10/14/2019, Q. Fan 17570 (holotype: SYS; isotype: IBSC, SYS); Q. Fan 13700 (paratype: SYS); Q. Fan 13701 (paratype: SYS); Q. Fan 13887 (paratype: SYS); Q. Fan 13900 (paratype: SYS); Q. Fan 13901 (paratype: SYS); Q. Fan 17540 (paratype: SYS); Q. Fan 17543 (paratype: SYS).

Euphorbia xianxialingensis F. Y. Zhang, W. Y. Xie \& Z. H. Chen (Euphorbiaceae) Journal of Zhejiang Forestry Science and Technology 40 (1): 82. 2020.

TYPE: CHINA (Zhejiang*), 7/14/2015, Z. H. Chen \& al. JS20150714022 (holotype: ZM; isotype: ZM); Z. H. Chen \& al. JS20160516008 (paratype: ZM); Z. H. Chen \& Z. X. Zheng JS19061202 (paratype: ZM).

Fargesia purpurea D. Z. Li \& Xia Y. Ye (Poaceae) PhytoKeys 170: 29. 2020.

TYPE: CHINA (Xizang*), 9/1/2015, Xia Y. Ye \& X. He YXY254-1 (holotype: KUN; isotype: KUN); Xia Y. Ye \& X. He YXY254-2 (paratype: KUN)

Fargesia viridis D. Z. Li \& Xia Y. Ye (Poaceae) PhytoKeys 170: 27. 2020.

TYPE: CHINA (Yunnan*), 8/24/2015, Xia Y. Ye YXY272 (holotype: KUN; isotype: KUN).

Ficus jingningensis X. D. Mei, Z. H. Chen \& G. Y. Li (Moraceae) Journal of Zhejiang Forestry Science and Technology 40 (5): 53.2020. TYPE: CHINA (Zhejiang*), 8/19/2019, X. D. Mei \& Z. H. Chen JN19081902 (holotype: ZM; isotype: ZM); G. Y. Li, L. Chen \& Z. H. Chen JN20071401 (paratype: ZM); G. Y. Li, L. Chen \& Z. H. Chen JN20071402 (paratype: ZM); Z. H. Chen, X. D. Mei \& G. Y. Li JN 20072801 (paratype: ZM); X. D. Mei, R. L. Liu \& Z. H. Chen JN20072803 (paratype: ZM).

Firmiana daweishanensis Gui L. Zhang \& J. Y. Xiang (Malvaceae) Phytotaxa 456 (2): 215. 2020.

TYPE: CHINA (Yunnan*), 3/1/2013, Gui L. Zhang 2013001 (holotype: SWFC-0072399; isotype: SWFC-0072400, KUN-1347143, KUN-1348014).

Flemingia weii T. V. Do \& X. F. Gao (Fabaceae) Phytotaxa 429 (1): 29. 2020.

TYPE: CHINA (Yunnan*), 12/10/1939, M. K. Li 1581 (holotype: KUN-0612135); W. Wang 10194 (paratype: KUN-0616222)

Frullania dasyueshanensis Schäf.-Verw. \& G. Winter bis (Lejeuneaceae) Frahmia 20: 2. 2020.

TYPE: CHINA (Taiwan*), 10/8/2016, A. Schäfer-Verwimp DY-106 (holotype: TAIM; isotype: FR, TAIE, JE, CAS).

Geum sunhangii D. G. Zhang, T. Deng, Z. Y. Lv \& Zhi M. Li (Rosaceae) PhytoKeys 156: 115. 2020.

TYPE: CHINA (Hubei*), 7/11/2011, D. G. Zhang zdg7313 (holotype: KUN).

Glochidion lanyuense Gang Yao \& S. X. Luo (Phyllanthaceae) PhytoKeys 159: 147. 2020.

TYPE: CHINA (Taiwan*), 9/16/1998, C. M. Wang 03521 (holotype: IBSC-0330741).

Gnetum chinense Y. Yang, Bing Liu \& S. Z. Zhang (Gnetaceae) PhytoKeys 148: 109. 2020.

TYPE: CHINA (Yunnan*), 3/31/2015, B. Liu, Y. Yang \& T. W. Xiao 2627 (holotype: PE).

Goodyera nanshanensis Xi L. Wang \& X. H. Jin (Orchidaceae) Phytotaxa 460 (4): 296. 2020.

TYPE: CHINA (Hunan*), 7/13/2019, X. H. Jin 23232 (holotype: PE).

Gymnosphaera henryi Y. H. Yan \& Rui Zhang bis (Pteridaceae) Phytotaxa 449 (1): 24. 2020.

TYPE: CHINA (Hainan*), 1/12/2019, Y. H. Yan Fern09770 (holotype: CSH; isotype: CSH, PE, IBSC).

Gymnosphaera saxicola S. Y. Dong \& Z. Y. Zuo (Cyatheaceae) Journal of Systematics and Evolution 10.1111/jse.12679: 9. 2020.

TYPE: CHINA (Yunnan*), 8/6/2018, S. Y. Dong 5015 (holotype: IBSC; isotype: IBSC); S. Y. Dong 5017 (paratype: IBSC); Z. Y. Zuo 2825 (paratype: KUN); Z. Y. Zuo 2927 (paratype: KUN).

Gymnostachyum morsei Y. F. Deng (Acanthaceae) Kew Bulletin 75 (59): 2. 2020.

TYPE: CHINA (Guangxi*), 4/2/2019, Y. F. Deng \& Y. F. Huang 27477 (holotype: IBSC; isotype: GXMI, HITBC, IBK, IBSC, K).

Hanceola suffruticosa Y. P. Chen, A. J. Paton \& C. L. Xiang (Lamiaceae) PhytoKeys 145: 133. 2020.

TYPE: CHINA (Yunnan*), 12/1/2018, Y. P. Chen \& L. Q. Jiang EM748 (holotype: KUN; isotype: K, KUN, PE). 
杜诚, 刘军, 叶文, 廖帅, 葛斌杰, 刘冰, 马金双 (2021) 中国植物新分类群、新名称 2020 年度报告. 生物多样性, 29, 10111020. http://www.biodiversity-science.net/CN/10.17520/biods.2021122

Hedychium mechukanum M. Sabu \& Hareesh (Zingiberaceae) The Gardens' Bulletin Singapore 72 (2): 292. 2020.

TYPE: CHINA (Xizang*), 9/19/2019, M. Sabu \& V. S. Hareesh 158761 (holotype: MBGH; isotype: CAL).

Hedyotis zhihaoana Huan C. Wang \& Xiao Lan Liu (Rubiaceae) Phytotaxa 461 (3): 168. 2020.

TYPE: CHINA (Yunnan*), 10/9/1979, Z. H. Hu, H. M. Yuan \& J. D. Zhang 7900570 (holotype: YUKU; isotype: YUKU); H. C. Wang 20110701(paratype: YUKU); G. D. Tao \& X. W. Li 39954 (paratype: HITBC, KUN); W. M. Zhu, J. J. He, S. G. Lu \& Z. Y. Liu 15381 (paratype: PYU); W. M. Zhu, J. J. He, S. G. Lu, \& Z. Y. Liu 15335 (paratype: PYU); H. C. Wang 20120803 (paratype: YUKU); G. D.Tao \& X. W. Li 39892 (paratype: HITBC, KUN).

Hemiboea yongfuensis Z. P. Huang \& Y. B. Lu (Gesneriaceae) Nordic Journal of Botany 38 (e02435): 3. 2020. TYPE: CHINA (Guangxi*), 10/10/2018, Q. Zhang 380846 (holotype: IBK; isotype: PE).

Henckelia siangensis Taram, D. Borah \& Tag (Gesneriaceae) PhytoKeys 160: 2. 2020.

TYPE: CHINA (Xizang*), 4/26/2019, O. Taku \& M. Taram 05001 (holotype: ASSAM; isotype: ARUN).

Hiptage lushuiensis S. P. Dong, K. Tan \& M. X. Ren (Rosaceae) Nordic Journal of Botany 38 (e02436): 2. 2020.

TYPE: CHINA (Yunnan*), 5/27/2018, S. P. Dong 177 (holotype: HUTB; isotype: HUTB, KUN); S. P. Dong 171 (paratype: HUTB).

Hoya gaoligongensis M. X. Zhao \& Y. H. Tan (Apocynaceae) Phytotaxa 459 (3): 220. 2020.

TYPE: CHINA (Yunnan*), 11/1/2018, M. X. Zhao, H. Y. Wang \& Z. J. Yin XHS-GCS-0685 (holotype: KUN; isotype: HITBC).

Hoya longicalyx Hui Wang bis \& E. F. Huang (Apocynaceae) Taiwania 65 (3): 354. 2020.

TYPE: CHINA (Yunnan*), 5/12/2019, E. F. Huang 1905003 (holotype: SZG; isotype: PE, KUN, TAI).

Hoya nyingchiensis Y. W. Zuo \& H. P. Deng (Apocynaceae) Phytotaxa 468 (1): 130. 2020.

TYPE: CHINA (Xizang*), 7/9/2015, Y. W. Zuo \& X. Z. Lan 542621150719680LY (holotype: SWCTU).

Ilex jiangmenensis Lei Jiang \& K. W. Xu (Aquifoliaceae) Phytotaxa 428 (2): 153. 2020.

TYPE: CHINA (Guangdong*), 3/27/2011, Lei Jiang \& al. GDS00201 (holotype: IBSC; isotype: KUN).

Impatiens bomiensis Y. Y. Cong \& Y. C. Peng (Balsaminaceae) Nordic Journal of Botany 38 (e02761): 2. 2020.

TYPE: CHINA (Xizang*), 8/17/2016, Y. Y. Cong 32486 (holotype: HNNU; isotype: HNNU).

Impatiens dasyvexilla Q. L. Gan \& Xin W. Li (Balsaminaceae) Novon 28 (4): 253. 2020.

TYPE: CHINA (Hubei*), 9/24/2019, Xin W. Li 191721 (holotype: HIB); Xin W. Li 191722 (paratype: HIB); Xin W. Li 191723 (paratype: HIB); Xin W. Li 191724 (paratype: HIB); Xin W. Li 191725 (paratype: HIB); Xin W. Li 191726 (paratype: HIB); Xin W. Li 191727 (paratype: HIB); Xin W. Li 191728 (paratype: HIB); Xin W. Li 208001 (paratype: HIB); Xin W. Li 208002 (paratype: HIB); Xin W. Li 208003 (paratype: HIB).

Impatiens gongchengensis Z. C. Lu, B. Pan \& Yan Liu (Balsaminaceae) Taiwania 65 (1): 1. 2020.

TYPE: CHINA (Guangxi*), 10/12/2018, Z. C. Lu \& Ying Qin LZC135 (holotype: IBK; isotype: IBK, PE); Ying Qin \& G. F. Mou LZC194 (paratype: IBK, PE).

Impatiens longlinensis S. X. Yu (Balsaminaceae) Systematic Botany 45 (4): 894. 2020.

TYPE: CHINA (Guangxi*), 8/1/2013, S. X. Yu 8087 (holotype: PE; isolectotype: IBK).

Impatiens macrantha S. X. Yu \& Ying Qin (Balsaminaceae) Nordic Journal of Botany 38 (e02784): 2. 2020.

TYPE: CHINA (Guangxi*), 10/16/2005, S. X. Yu 3755 (holotype: PE; isotype: GXMI); Debao Med. Pl. Exped. 451024170927002LY (paratype: IBK); Debao Med. Pl. Exped. QY20181030001 (paratype: IBK).

Impatiens plicatisepala C. Y. Zou, Yan Liu \& S. X. Yu (Balsaminaceae) Taiwania 65 (4): 544. 2020.

TYPE: CHINA (Guangxi*), 9/24/2016, C. Y. Zou \& Ying Qin 450329160809024LY (holotype: IBK; isotype: PE); C. Y. Zou \& Jing Liu $450329160925002 L Y$ (paratype: IBK).

Impatiens pseudolongipes Gogoi, Sherpa \& Borah (Balsaminaceae) Phytotaxa 429 (3): 218. 2020.

TYPE: CHINA (Xizang*), 7/24/2013, R. Gogoi 30521 (holotype: CAL; isotype: CAL).

Impatiens quintadecimacopii G. W. Hu \& Q. F. Wang (Balsaminaceae) Plant Science Journal 38 (4): 440. 2020.

TYPE: CHINA (Yunnan*), 7/28/2019, G. W. Hu, S. Peng \& J. J. Wang HGW-1200 (holotype: HIB; isotype: HIB); G. W. Hu \& al. HGW0911 (paratype: HIB); G. W. Hu \& al. HGW-2022 (paratype: HIB).

Impatiens shenglanii Q. L. Gan \& Xin W. Li (Balsaminaceae) Annales Botanici Fennici 57 (4-6): 218. 2020. 
杜诚, 刘军, 叶文, 廖帅, 葛斌杰, 刘冰, 马金双 (2021) 中国植物新分类群、新名称 2020 年度报告. 生物多样性, 29, 10111020. http://www.biodiversity-science.net/CN/10.17520/biods.2021122

TYPE: CHINA (Hubei*), 8/2/2019, Xin W. Li 191001 (holotype: HIB); Xin W. Li 191002 (paratype: HIB); Xin W. Li 191003 (paratype: HIB); Xin W. Li 191004 (paratype: HIB); Xin W. Li 191005 (paratype: HIB); Xin W. Li 191006 (paratype: HIB).

Impatiens shiyomiensis Hareesh \& M. Sabu (Balsaminaceae) Phytotaxa 437 (5): 294. 2020.

TYPE: CHINA (Xizang*), 9/18/2019, M. Sabu \& V. S. Hareesh 158764 (holotype: CAL; isotype: CAL, MBGH).

Impatiens tirbinensis Hareesh \& M. Sabu (Balsaminaceae) Phytotaxa 437 (5): 291. 2020.

TYPE: CHINA (Xizang*), 9/19/2019, M. Sabu \& V. S. Hareesh 158721 (holotype: CAL; isotype: CAL, MBGH).

Impatiens wuyiensis Jian S. Wang, Y. F. Lu \& X. F. Jin (Balsaminaceae) Botanical Studies (Taipei) 61 (29): 3. 2020.

TYPE: CHINA (Fujian*), 5/23/2018, X. F. Jin, Y. F. Lu \& Jian S. Wang 4158 (holotype: ZM-ZMNH0068001; isotype: HTC-0021906, KUN, PE, ZJFC); X. F. Jin, Y. F. Lu \& Jian S. Wang 4156 (paratype: ZJFC, ZM); X. F. Jin, Y. F. Lu \& Jian S. Wang 4157 (paratype: PE, ZJFC, ZM); X. F. Jin, Y. F. Lu \& Jian S. Wang 4165 (paratype: ZM); X. F. Jin, Y. F. Lu \& Jian S. Wang 4166 (paratype: KUN, PE, ZM); X. F. Jin, Y. F. Lu \& Jian S. Wang 4167 (paratype: KUN, PE, ZM); X. F. Jin, Y. F. Lu \& Jian S. Wang 4375 (paratype: ZJFC, ZM); X. F. Jin \& Y. F. Lu s.n. (paratype: ZM); Y. F. Lu 186 (paratype: ZM).

Impatiens zhuxiensis Q. L. Gan \& Xin W. Li (Balsaminaceae) Nordic Journal of Botany 38 (e02686): 2. 2020.

TYPE: CHINA (Hubei*), 9/23/2019, Xin W. Li 191662 (holotyp: HIB); Xin W. Li 91663 (paratype: HIB); Xin W. Li 191664 (paratype: HIB); Xin W. Li 191665 (paratype: HIB); Xin W. Li 191666 (paratype: PE); Xin W. Li 191667 (paratype: PE); Xin W. Li 191668 (paratype: KUN); Xin W. Li 191669 (paratype: IBK).

Indigofera yuanjiangensis X. F. Gao \& X. L. Zhao (Fabaceae) Phytotaxa 455 (3): 235. 2020.

TYPE: CHINA (Yunnan*), 6/2/2018, X. L. Zhao ZXL389-1 (holotype: CDBI; isotype: CDBI).

Involucrella lithophila M. D. Yuan \& R. J. Wang (Rubiaceae) Phytotaxa 464 (1): 60. 2020.

TYPE: CHINA (Guangxi*), 8/17/2019, S. Chen YS49 (holotype: IBSC-0861073; isotype: IBSC-0861072, IBSC-0861071); Y. D. Peng, L. L. Mo \& Y. Y. Xie 451402150914024LY (paratype: GXMG); Guangxi Provincial Museum 6425 (paratype: IBSC); K. J. Yan 80621 (paratype: GXMI); Nonggang Investigation Team 20450 (paratype: GXMI); Nonggang Investigation Team 11212 (paratype: IBK); $R$. J. Wang 1632 (paratype: IBSC); K. Zhang 00326 (paratype: IBSC); K. Zhang 00349 (paratype: IBSC); B. Y. Huang, H. Z. Lv \& Y. Y. Xie 451422131028003LY (paratype: GXMG); R. J. Wang 2686 (paratype: IBSC); X. M. Lu \& C. C. Huang 11422 (paratype: GXMG); X. M. Lu \& C. C. Huang 11606 (paratype: GXMG); M. X. Luo 20711 (paratype: GXMG); R. J. Wang 2596 (paratype: IBSC); R. J. Wang 2597 (paratype: IBSC); G. B. Jiang, X. X. Zhou \& M. D. Yuan 1491 (paratype: IBSC); Mashan Investigation Team 2-70 (paratype: GXMI),

Iris dabashanensis C. A. Wilson (Iridaceae) PhytoKeys 161: 49. 2020.

TYPE: CHINA (Sichuan*), 5/3/2001, D. Probst CPC3.5.01.3 (holotype: UC; isotype: E, PE).

Iris probstii C. A. Wilson (Iridaceae) PhytoKeys 161: 53. 2020.

TYPE: CHINA (Guizhou*), 7/24/2003, D. Probst CPC24.7.03.1 (holotype: UC; isotype: E, PE).

Isodon brevipedunculatus Y. P. Chen \& C. L. Xiang (Lamiaceae) Plant Diversity 43 (x): 5/9. 2020.

TYPE: CHINA (Guangdong*, Hunan), 10/25/2019, Y. P. Chen \& Y. Zhao EM1381 (holotype: KUN; isotype: A, K, KUN, P, PE, W).

Isotrema brevilimbum X. X. Zhu, Jun Wang tris \& F. Cao (Aristolochiaceae) PhytoKeys 152: 16. 2020.

TYPE: CHINA (Guizhou*), 8/5/2018, X. X. Zhu \& al. ZXX18217 (holotype: CSH-0172289; isotype: CSH, KUN).

Isotrema hei Lei Cai \& X. X. Zhu (Aristolochiaceae) Annales Botanici Fennici 57 (1-3): 125. 2020.

TYPE: CHINA (Yunnan*), 5/12/2018, X. X. Zhu ZXX18072 (holotype: CSH; isotype: CSH, KUN); Lei Cai \& al. CL115 (paratype: KUN); X. X. Zhu ZXX18065 (paratype: KUN).

Jasminum chiae Kai Zhang \& D. X. Zhang (Oleaceae) Nordic Journal of Botany 38 (e02857): 3. 2020.

TYPE: CHINA (Yunnan*), 8/3/2020, Kai Zhang 01241 (holotype: IBSC; isotype: HITBC, IBSC); Kai Zhang, M. S. Wu 00612 (paratype: IBSC); Kai Zhang, M. S. Wu 00617 (paratype: IBSC); Kai Zhang 01220 (paratype: IBSC).

Jasminum parceflorum Kai Zhang \& D. X. Zhang (Oleaceae) PhytoKeys 146: 110. 2020.

TYPE: CHINA (Yunnan*), 7/18/2018, Kai Zhang \& M. S. Wu 00581 (holotype: IBSC; isotype: IBSC, HITBC).

Lepisorus youxingii R. H. Jiang \& X. C. Zhang (Polypodiaceae) Phytotaxa 437 (2): 102. 2020.

TYPE: CHINA (Guangxi*), 8/12/2019, R. H. Jiang \& al. 13004 (holotype: PE); R. H. Jiang \& al. 13003 (paratype: PE); R. H. Jiang \& al. JRH2503 (paratype: IBK)

Leptochilus gracilis Z. L. Liang, Liang Zhang \& Li Bing Zhang (Polypodiaceae) Phytotaxa 468 (1): 117. 2020.

TYPE: CHINA (Yunnan*), 9/9/2019, Z. L. Liang, Y. M. Shui, W. H. Chen \& Z. Y. Yu 607 (holotype: KUN-1493479; isotype: CDBI). 
杜诚, 刘军, 叶文, 廖帅, 葛斌杰, 刘冰, 马金双 (2021) 中国植物新分类群、新名称 2020 年度报告. 生物多样性, 29, 10111020. http://www.biodiversity-science.net/CN/10.17520/biods.2021122

Liparis mae X. D. Tu, M. Z. Huang \& Ming H. Li (Orchidaceae) Phytotaxa 435 (3): 236. 2020.

TYPE: CHINA (Guangxi*), 5/1/2018, FJFC 0676 (holotype: FJFC; isotype: FJFC).

Lonicera omissa P. L. Chiu, Z. H. Chen \& Y. L. Xu (Caprifoliaceae) Journal of Hangzhou Normal University (Natural Science Edition) 19 (3): 253. 2020.

TYPE: CHINA (Zhejiang*), 5/11/2018, Y. L. Xu, X. Cai \& J. L. Liu Xu517 (holotype: ZM; isotype: ZM).

Luisia yunnanensis Jie Huang tris \& D. H. Peng (Orchidaceae) Phytotaxa 475 (1): 54. 2020.

TYPE: CHINA (Yunnan*), 4/16/2020, Y. Y. Yu 20200416-23 (holotype: FAFU-20200416-23).

Lycoris tsinlingensis P. C. Zhang, Yi Jun Lu \& Ting Wang bis (Amaryllidaceae) Annales Botanici Fennici 57 (4-6): 193. 2020.

TYPE: CHINA (Shaanxi*), 7/16/2014, P. C. Zhang 028 (holotype: HHBG); P. C. Zhang 030 (paratype: HHBG).

Lysimachia daqiaoensis G. D. Tang \& R. Z. Huang (Primulaceae) Phytotaxa 430 (1): 41.2020.

TYPE: CHINA (Guangdong*), 5/14/2019, G. D. Tang, R. Z. Huang, M. Liao \& W. Han GD190501 (holotype: CANT; isotype: IBSC); G. D. Tang, W. Han GD190502 (paratype: IBSC).

Lysimachia porcatisepala S. R. Yi (Primulaceae) Phytotaxa 434 (1): 118. 2020.

TYPE: CHINA (Chongqing*), 5/15/2019, S. R. Yi 8189 (holotype: KUN; isotyepe: KUN, PE).

Lysimachia xiangxiensis D. G. Zhang, C. Mou \& Yu Wu (Primulaceae) PhytoKeys 140: 27. 2020.

TYPE: CHINA (Hunan*), 8/26/2019, D. G. Zhang 0826075 (holotype: JIU; isotype: JIU).

Lysionotus coccinus G. W. Hu \& Q. F. Wang (Gesneriaceae) Nordic Journal of Botany 38 (e02912): 2. 2020.

TYPE: CHINA (Yunnan*), 7/27/2019, G. W. Hu, S. Peng \& J. J. Wang HGW-1180 (holotype: HIB; isotype: HIB).

Maclura montana Z. P. Lei, G. Y. Li \& Z. H. Chen (Moraceae) Journal of Zhejiang Forestry Science and Technology 40 (6): 62.2020.

TYPE: CHINA (Zhejiang*), 7/15/2020, Z. H. Chen, G. Y. Li \& S. Z. Hu JN20071509 (holotype: ZM; isotype: ZM); Z. H. Chen, G. Y. Li \& S. Z. Hu JN20071507 (paratype: ZM); Z. H. Chen, G. Y. Li \& S. Z. Hu JN20071510 (paratype: ZM); Z. H. Chen, G. Y. Li \& S. Z. Hu JN20071511 (paratype: ZM); Z. P. Lei, X. Liu \& S. Z. Hu JN20072805 (paratype: ZM); R. L. Liu, G. Y. Li \& X. D. Mei JN20072806 (paratype: ZM); L. S. Lin \& X. D. Mei JN20072807 (paratype: ZM); Z. H. Chen, W. Y. Xie \& S. Z. Hu JN17080209 (paratype: ZM); Z. H. Chen, R. L. Liu \& S. Z. Hu JN14080252 (paratype: ZM); Z. H. Chen \& G. Y. Li JN20101404; Z. H. Chen \& G. Y. Li JN20101405 (paratype: ZM).

Maclura orientalis G. Y. Li, W. Y. Xie \& Z. H. Chen (Moraceae) Journal of Zhejiang Forestry Science and Technology 40 (5): 56.2020. TYPE: CHINA (Zhejiang*), 7/29/2020, Z. P. Lei, G. Y. Li \& S. Z. Hu JN20072901 (holotype: ZM; isotype: ZM); J. P. Zhong \& W. Y. Xie LD16073102 (paratype: ZM); Z. H. Chen \& al. JY-002 (paratype: ZM); Z. H. Chen \& al. LQ19061604 (paratype: ZM); Editorial Committee of Flora of medicinal plants in Zhejiang 1326 (paratype: ZM); Editorial Committee of Flora of medicinal plants in Zhejiang 2040 (paratype: ZM); Z. H. Chen \& al. JN16080118 (paratype: ZM); G. Y. Li, Z. H. Chen \& F. Chen JN170801-06 (paratype: ZM); Z. H. Chen \& al. JN19072504 (paratype: ZM); Z. H. Chen, G. Y. Li \& S. Z. Hu JN20071504 (paratype: ZM); Z. H. Chen, G. Y. Li \& S. Z. Hu JN20071505 (paratype: ZM); Z. P. Lei, X. Liu \& S. Z. Hu JN20072903 (paratype: ZM); Z. H. Chen \& al. JN18062007 (paratype: ZM); Z. H. Chen, G. Y. Li \& S. Z. Hu JN20071502 (paratype: ZM); Z. H. Chen, S. Z. Hu \& X. D. Mei N19081804 (paratype: ZM); Z. H. Chen \& al. JN18051905 (paratype: ZM); X. D. Mei JN20072401 (paratype: ZM); W. Y. Xie \& al. QT1505090 (paratype: ZM); G. Y. Li, D. D. Ma \& al., YJ009 (paratype: ZJFC).

Meeboldia linearis X. L. Guo, W. Gou \& X. J. He (Apiaceae) Nordic Journal of Botany 38 (e02635): 6. 2020.

TYPE: CHINA (Xizang*), 9/29/2018, X. L. Guo \& W. Gou s.n. (holotype: SZ; isotype: SZ); Xizang Herb. Med. Exped. 4427 (paratype: $\mathrm{PE})$.

Meehania zheminensis A. Takano, Pan Li \& G. H. Xia (Lamiaceae) Plants 9 (1159): 9. 2020.

TYPE: CHINA (Fujian, Zhejiang*), 4/10/2020, Pan Li LP207976 (holotype: HZU; isotype: HHBG, HYO, KYO, KUN, PE, TI, TNS, $\mathrm{ZM})$.

Mitreola crystallina Y. M. Shui \& W. H. Chen (Loganiaceae) Phytotaxa 471 (2): 140. 2020.

TYPE: CHINA (Yunnan*), 2/1/2005, Y. M. Shui \& W. H. Chen. 43869 (holotype: KUN); W. H. Chen \& M. D. Zhang 43869 (paratype: KUN); L. Wu \& al. 5740 (paratype: CSFI-069616); L. Wu \& al. 5809 (paratype: CSFI-069617); C. Liu, M. J. Feng, X. J. Hu, C. H. Li \& M. F. Long 20CS19487 (paratype: KUN).

Mucuna guangxiensis K. W. Jiang \& Y. Feng Huang (Fabaceae) Phytotaxa 433 (2): 149. 2020.

TYPE: CHINA (Guangxi*), 4/13/2019, Y. Feng Huang \& Y. D. Huang 31405 (holotype: CSH; isotype: CSH, GXMI, SWFC); Y. D. Huang 01 (paratype: CSH); Y. D. Huang 01 (paratype: CSH, GXMI).

Oberonia integrilabris Yu L. Li \& F. W. Xing (Orchidaceae) Phytotaxa 432 (1): 105. 2020. 
杜诚, 刘军, 叶文, 廖帅, 葛斌杰, 刘冰, 马金双 (2021) 中国植物新分类群、新名称 2020 年度报告. 生物多样性, 29, 10111020. http://www.biodiversity-science.net/CN/10.17520/biods.2021122

TYPE: CHINA (Yunnan*), 7/10/2012, Y. Tong 237 (holotype: IBSC; isotype: IBSC).

Omphalotrigonotis taishunensis Shao Z. Yang, W. W. Pan \& J. P. Zhong (Boraginaceae) Journal of Hangzhou Normal University (Natural Science Edition) 19 (3): 258. 2020.

TYPE: CHINA (Zhejiang*), 5/25/2017, W. W. Pan \& J. P. Zhong WYL2017052501 (holotype: ZJFI; isotype: ZJFI).

Onosma fuyunensis Yi He \& Q. R. Liu (Boraginaceae) PhytoKeys 144: 13. 2020.

TYPE: CHINA (Xinjiang*), 7/7/2017, Y. He \& Y. Zhou BNU2017XJ133 (holotype: BNU-0041549; isotype: BNU, PE).

Oreocharis aimodisca Lei Cai, Z. L. Dao \& F. Wen (Gesneriaceae) PhytoKeys 162: 3. 2020.

TYPE: CHINA (Yunnan*), 9/10/2019, L. Cai \& P. Zhang CL275 (holotype: KUN; isotype: KUN, IBK).

Oreocharis argentifolia Lei Cai \& Z. L. Dao (Gesneriaceae) Nordic Journal of Botany 38 (e02699): 2. 2020.

TYPE: CHINA (Yunnan*), 9/12/2019, Lei Cai CL305 (holotype: KUN; isotype: KUN).

Oreocharis eriocarpa W. H. Chen \& Y. M. Shui (Gesneriaceae) PhytoKeys 157: 89. 2020.

TYPE: CHINA (Yunnan*), 7/30/2013, Y. M. Shui, D. M. He \& al. B2013-304 (holotype: KUN; isotype: PE); Y. M. Shui \& al. B2015315A (paratype: KUN); D. M. He \& T. Zhang WSLJS558 (paratype: KUN); T. Zhang, D. M. He \& Y. F. Feng $18 C S 17589$ (paratype: KUN).

Oreocharis flavovirens Xin Hong (Gesneriaceae) PhytoKeys 157: 102. 2020.

TYPE: CHINA (Gansu*), 9/5/2018, Xin Hong HX18090510 (holotype: IBK; isotype: PE); Y. F. Gao \& al. WF19092401 (paratype: AHU).

Oreocharis fulva W. H. Chen \& Y. M. Shui (Gesneriaceae) PhytoKeys 157: 91. 2020.

TYPE: CHINA (Yunnan*), 9/13/2013, Y. M. Shui \& al. B2013-579 (holotype: KUN; isotype: KUN, PE).

Oreocharis jasminina S. J. Ling, F. Wen \& M. X. Ren (Gesneriaceae) PhytoKeys 157: 102. 2020.

TYPE: CHINA (Hainan*), 11/26/2018, S. J. Ling 2018112601 (holotype: HUTB; isotype: HUTB, KUN).

Oreocharis lacerata W. H. Chen \& Y. M. Shui (Gesneriaceae) PhytoKeys 157: 93. 2020.

TYPE: CHINA (Yunnan*), 8/5/2003, S. S. Zi 208 (holotype: KUN; isotype: PE); S. S. Zi 261 (paratype: KUN, PE); Y. L. Li YDDXS 1137 (paratype: KUN).

Oreocharis longipedicellata Lei Cai \& F. Wen (Gesneriaceae) PhytoKeys 162: 7. 2020.

TYPE: CHINA (Yunnan*), 8/24/2019, F. Wen WF190824-01 (holotype: KUN; isotype: IBK).

Oreocharis wenshanensis W. H. Chen \& Y. M. Shui (Gesneriaceae) PhytoKeys 157: 96. 2020.

TYPE: CHINA (Yunnan*), 7/27/1993, Y. M. Shui 3126 (holotype: KUN; isotype: PE); Y. M. Shui, D. M. He \& al. B2012-099 (paratype: KUN); Y. M. Shui \& B. Xiao B2013-100C (paratype: KUN).

Oreocharis wumengensis Lei Cai \& Z. L. Dao (Gesneriaceae) PhytoKeys 157: 114. 2020.

TYPE: CHINA (Yunnan*), 8/3/2018, Lei Cai CL198 (holotype: KUN; isotype: KUN).

Oreorchis wumanae T. P. Lin (Orchidaceae) Taiwania 65 (4): 463. 2020.

TYPE: CHINA (Taiwan*), 3/16/2020, C. H. Liu s.n. (holotype: TAI-289760).

Oxalis shibeishanensis Huan C. Wang \& Y. Tian (Oxalidaceae) Taiwania 65 (3): 354. 2020.

TYPE: CHINA (Yunnan*), 3/24/2019, Huan C. Wang, F. Yang, Q. P. Wang \& Y. R. Li SB5788 (holotype: YUKU-02070800; isotype: YUKU-02070801, YUKU-02070802, YUKU-02070803).

Oxytropis shennongjiaensis D. G. Zhang, J. T. Chen, T. Deng \& H. Sun (Fabaceae) PhytoKeys 149: 120. 2020.

TYPE: CHINA (Hubei*), 6/9/2019, D. G. Zhang \& Q. Liu 19060901 (holotype: KUN-1347953; isotype: JIU).

Paraboea brevipedunculata Y. M. Shui \& W. H. Chen (Gesneriaceae) PhytoKeys 157: 212. 2020.

TYPE: CHINA (Yunnan*), 8/26/2005, Y. M. Shui \& W. H. Chen B2017-1342 (holotype: KUN); Y. M. Shui \& W. H. Chen B2013-094 (paratype: KUN); Y. M. Shui \& W. H. Chen B2017-1342 (paratype: KUN); Y. M. Shui \& W. H. Chen B2018-021 (paratype: KUN).

Paraboea dolomitica Z. Yu Li, X. G. Xiang \& Z. Y. Guo (Gesneriaceae) PhytoKeys 153: 41. 2020.

TYPE: CHINA (Guizhou*), 5/2/2017, Z. Y. Guo 20170047 (holotype: PE; isotype: QNUN, PE).

Paraboea myriantha Y. M. Shui \& W. H. Chen (Gesneriaceae) PhytoKeys 157: 209. 2020. 
杜诚, 刘军, 叶文, 廖帅, 葛斌杰, 刘冰, 马金双 (2021) 中国植物新分类群、新名称 2020 年度报告. 生物多样性, 29, 10111020. http://www.biodiversity-science.net/CN/10.17520/biods.2021122

TYPE: CHINA (Yunnan*), 4/30/2017, Y. M. Shui \& al. 44536 (holotype: KUN); Y. M. Shui \& al. 15105 (paratype: KUN); Y. M. Shui \& al. 20595 (paratype: KUN); Y. M. Shui \& al. 20946 (paratype: KUN); Y. M. Shui, B. Xiao, J. Wang \& al. B2013-528 (paratype: KUN); Y. M. Shui \& al. 30261 (paratype: KUN); Y. M. Shui \& al. s.n. (paratype: KUN); Y.M. Shui \& al. 16118 (paratype: KUN); Y. M. Shui \& al. B2018-183 (paratype: KUN).

Paraboea nanxiensis Lei Cai \& Gui L. Zhang (Gesneriaceae) Guihaia 40 (10): 1424. 2020.

TYPE: CHINA (Yunnan*), 7/14/2017, Gui L. Zhang \& al. CL2017085 (holotype: KUN; isotype: KUN, IBK).

Paraphlomis kuankuoshuiensis R. B. Zhang, Tan Deng \& C. B. Ma (Lamiaceae) PhytoKeys 139: 15. 2020.

TYPE: CHINA (Guizhou*), 7/22/2019, R. B. Zhang ZRB1509 (holotype: ZY; isotype: IBK); R. B. Zhang ZRB1575 (paratype: ZY).

Pedicularis khoiyangii D. Borah \& R. Kr. Singh (Orobanchaceae) Phytotaxa 430 (4): 288. 2020.

TYPE: CHINA (Xizang*), 8/23/2019, D. Borah 0124 (holotype: CAL; isotype: ASSAM).

Peliosanthes ligniradicis N. Tanaka, M.T aram \& D. Borah (Asparagaceae) Phytotaxa 438 (1): 44. 2020.

TYPE: CHINA (Xizang*), 11/16/2018, M. Taram \& D. Borah 431 (holotype: ASSAM; isotype: ARUN); M. Taram \& D. Borah 651 (paratype: ASSAM).

Pertya ferruginea Cai F. Zhang (Asteraceae) Phytotaxa 474 (3): 242. 2020.

TYPE: CHINA (Fujian, Jiangxi, Zhejiang*), 11/22/2008, Cai F. Zhang 1865 (holotype: PE; isotype: HIB, PE).

Pertya huangii Huan C. Wang \& Q. P. Wang (Asteraceae) Annales Botanici Fennici 57 (1-3): 87. 2020.

TYPE: CHINA (Yunnan*), 1/18/2018, Huan.C. Wang \& al. YM3040 (holotype: YUKU; isotype: YUKU); Huan.C. Wang \& al. YM3041 (paratype: YUKU); Huan.C. Wang \& al. YM7998 (paratype: YUKU).

Petrocodon chishuiensis Z. B. Xin, F. Wen \& S. B. Zhou (Gesneriaceae) Taiwania 65 (2): 181. 2020.

TYPE: CHINA (Guizhou*), 10/1/2010, F. Wen F.W.-Ges20101001 (holotype: IBK; isotype: IBK, TAI).

Petrocodon ionophyllus F. Wen, S. Li \& B. Pan (Gesneriaceae) Rheedea 30 (1): 151. 2020.

TYPE: CHINA (Guangxi*), 4/8/2013, Bo Pan \& al. BP P0783 (holotype: IBK; isotype: IBK, KUN).

Petrocodon luteoflorus Lei Cai \& F. Wen (Gesneriaceae) PhytoKeys 157: 169. 2020.

TYPE: CHINA (Guizhou*), 8/23/2018, Lei Cai \& al. CL193 (holotype: KUN; isotype: KUN, IBK).

Petrocodon rubiginosus Y. G. Wei \& R. L. Zhang (Gesneriaceae) PhytoKeys 157: 176. 2020.

TYPE: CHINA (Guangxi*), 11/15/2015, F. Wen \& al. WF151112-01 (holotype: IBK; isotype: IBK).

Petrocodon wenshanensis Xin Hong, W. H. Qin \& F. Wen (Gesneriaceae) PhytoKeys 157: 185. 2020.

TYPE: CHINA (Guangxi*), 6/14/2019, F. Wen \& al. WF170807-06 (holotype: IBK; isotype: AHU).

Petrocosmea chiwui M. Q. Han, H. Jiang \& Yan Liu (Gesneriaceae) Nordic Journal of Botany 38 (e02847): 2. 2020.

TYPE: CHINA (Yunnan*), 9/24/2017, M. Q. Han HMQ 1620 (holotype: IBK; isotype: PE, YAF); W. C. Wang 84475 (paratype: IBSC, KUN, PE, WUK).

Petrocosmea nanchuanensis Z. Y. Liu, Z. Yu Li \& Z. J. Qiu (Gesneriaceae) PhytoKeys 157: 138. 2020.

TYPE: CHINA (Chongqing*), 9/20/2002, S. J. Ling 2018112601 (holotype: HUTB; isotype: HUTB, KUN); R. H. Liang 2007010 (paratype: PE); Z. J. Qiu QZJ-20131082 (paratype: SZG); S. Z. Zhang 20121077 (paratype: SZG); Z. Y. Liu 500119-1582ly (paratype: IMC); Z. Y. Liu 500119-1595ly (paratype: IMC).

Petrocosmea rotundifolia M. Q. Han, H. Jiang \& Yan Liu (Gesneriaceae) Nordic Journal of Botany 38 (e02847): 2. 2020.

TYPE: CHINA (Yunnan*), 9/24/2017, M. Q. Han HMQ 1621 (holotype: IBK; isotype: PE, YAF); H. Jiang, J. Li and Q. Cao 7980 (paratype: YAF).

Peucedanum huangshanense Lu Q. Huang, H. S. Peng \& S. S. Chu (Apiaceae) Phytotaxa 430 (1): 21. 2020.

TYPE: CHINA (Anhui*), 8/20/2018, H. S. Peng 08201 (holotype: ACM; isotype: ACM); Chu \& Chen 102701 (paratype: ACM); Chu \& Chen102703 (paratype: ACM); Chu \& Han QH080205 (paratype: ACM); Chu \& Han QH080217 (paratype: ACM).

Phedimus yangshanicus Z. Chao (Crassulaceae) Phytotaxa 429 (2): 153. 2020.

TYPE: CHINA (Guangdong*), 4/6/2019, Z. Chao 2019040601 (holotype: Herbarium of Southern Medical University).

Phoebe hekouensis Bing Liu, W. Y. Jin, L. N. Zhao \& Y. Yang (Lauraceae) PhytoKeys 140: 102. 2020.

TYPE: CHINA (Yunnan*), 4/5/2014, Bing Liu, Y. Yang, Q. W. Lin, L. Jiang \& X. J. Li 1988 (holotype: PE; isotype: PE). 
杜诚, 刘军, 叶文, 廖帅, 葛斌杰, 刘冰, 马金双 (2021) 中国植物新分类群、新名称 2020 年度报告. 生物多样性, 29, 10111020. http://www.biodiversity-science.net/CN/10.17520/biods.2021122

Phytolacca yunnanensis X. H. Li \& Wen Zhou (Phytolaccaceae) Phytotaxa 446 (1): 51. 2020.

TYPE: CHINA (Yunnan*), 10/16/2018, X. H. Li \& Wen Zhou 181005 (holotype: NAU).

Piper lanyuense K. N. Kung \& Kun C. Chang (Piperaceae) Annales Botanici Fennici 57 (1-3): 93. 2020.

TYPE: CHINA (Taiwan*), 7/20/2009, K. N. Kung \& al. 1118 q (holotype: CHIA; isotype: TAIF); C. E. Chang 20009 (paratype: HAST); W. P. Leu 2153 (paratype: HAST); C. E. Chang 5563 (paratype: PPI); Huang \& Ku 5315 (paratype: TAI); S. Y. Lu 25476 (paratype: HAST); P. F. Lu 10829 (paratype: TAIF); C. H. Ou \& al. 9340 (paratype: TCB); T. Y. A. Yang 2679 (paratype: TNM).

Piper semi-transparens C. Y. Hao \& Y. H. Tan (Piperaceae) Phytotaxa 429 (3): 194. 2020.

TYPE: CHINA (Hainan*), 4/9/2015, C. Y. Hao 2015028 (holotype: HITBC; isotype: HITBC).

Plagiochila xerophila Bakalin \& Vilnet (Plagiochilaceae) Plant Ecology and Evolution 153 (1): 127. 2020.

TYPE: CHINA (Sichuan*), 10/14/2017, V.A. Bakalin \& K.G. Klimova China-46-2-17 (holotype: VGBI; isotype: KPABG).

Platanthera jiuwanshanensis Ying Qin \& Yan Liu (Orchidaceae) Phytotaxa 436 (1): 72. 2020.

TYPE: CHINA (Guangxi*), 9/16/2018, Ying Qin QY20180916002 (holotype: IBK; isotype: IBK); Ying Qin QY20190903001 (paratype: IBK).

Polygonatum daminense H. J. Yang \& D. F. Cui (Asparagaceae) Phytotaxa 449 (3): 290. 2020.

TYPE: CHINA (Zhejiang*), 5/4/2019, H. J. Yang 201902 (holotype: CANT; isotype: CANT).

Primula qiupuensis J. W. Shao (Primulaceae) Phytotaxa 441 (2): 177. 2020.

TYPE: CHINA (Anhui*), 3/16/2015, J. W. Shao \& J. Liu 20150316-2 (holotype: ANUB-002324; isotype: ANUB-002326, ANUB002321, ANUB-002320, ANUB-002319, ANUB-002315); J. W. Shao \& W. Zhang 20160409-1 (paratype: ANUB-002328); J. W. Shao \& J. Liu 20150313-1 (paratype: ANUB-002314); J. W. Shao \& J. Liu 20150314-2 (paratype: ANUB-002322); J. W. Shao \& J. Liu 20150313-2 (paratype: ANUB-002323); J. W. Shao \& J. Liu 20150313-1 (paratype: ANUB-002318, ANUB-002327); J. W. Shao \& W. Zhang 20160309-1 (paratype: ANUB-002316); J. W. Shao \& J. Liu 20150316-1 (paratype: ANUB-002325); J. W. Shao \& W. Zhang 20160410-1 (paratype: ANUB-002329).

Primula sunhangii T. Deng, D. G. Zhang \& Jiao Sun (Primulaceae) PhytoKeys 156: 106. 2020.

TYPE: CHINA (Hubei*), 6/5/2011, D. G. Zhang \& al. Zdg20110605023 (holotype: KUN; isotype: JIU).

Primulina flexusa F. Wen, T. Peng \& B. Pan (Gesneriaceae) PhytoKeys 159: 62. 2020.

TYPE: CHINA (Guizhou*), 5/15/2016, Bo Pan \& al. PB160425-01 (holotype: IBK; isotype: IBK).

Primulina huangjiniana W. B. Liao, Q. Fan \& C. Y. Huang (Gesneriaceae) Guihaia 40 (10): 1432. 2020.

TYPE: CHINA (Guangdong*), 4/20/2018, Q. Fan \& Y. S. Huang 16997 (holotype: SYS; isotype: IBK, IBSC, SYS); Q. Fan \& Y. S. Huang 16995 (paratype: SYS); Q. Fan 17003 (paratype: SYS).

Primulina inflata Li H. Yang \& M. Z. Xu (Gesneriaceae) Taiwania 65 (2): 163.2020.

TYPE: CHINA (Guangdong*), 4/13/2019, Li H. Yang YLH1024 (holotype: IBSC-0849964; isotype: IBSC-0849965, IBK).

Primulina jiuyishanica Kun Liu bis, D. C. Meng \& Z. B. Xin (Gesneriaceae) PhytoKeys 162: 39. 2020.

TYPE: CHINA (Guangdong*), 4/14/2017, Kun Liu 20170414-01 (holotype: IBK; isotype: IBK, KUN).

Primulina qintangensis Z. B. Xin, W. C. Chou \& F. Wen (Gesneriaceae) Acta Botanica Boreali-Occidentalia Sinica 40 (8): 1425.2020. TYPE: CHINA (Guangxi*), 1/9/2019, W. C. Chou \& al. 190109-01 (holotype: IBK; isotype: IBK).

Primulina spiradiclioides Z. B. Xin \& F. Wen (Gesneriaceae) Annales Botanici Fennici 57 (4-6): 245. 2020.

TYPE: CHINA (Guangxi*), 7/23/2016, F. Wen \& al. WF20160723-01 (holotype: IBK; isotype: IBK).

Primulina titan Z. B. Xin, W. C. Chou \& F. Wen (Gesneriaceae) Journal of Botanical Research 2 (3): 2. 2020.

TYPE: CHINA (Guangxi*), 5/9/2020, Z. B. Xin \& al. XZB20200509-01 (holotype: IBK; isotype: IBK).

Primulina yulinensis Ying Qin \& Yan Liu (Gesneriaceae) Taiwania 65 (1): 5.2020.

TYPE: CHINA (Guangxi*), 6/18/2017, Ying Qin \& al. QY20170618001 (holotype: IBK; isotype: IBK, PE); Ying Qin \& al. QY20170618002 (paratype: IBK); Ying Qin \& al. JJZ025 (paratype: IBK); Ying Qin \& al. JJZ067 (paratype: IBK); Yan Liu \& al. JJZ069 (paratype: IBK).

Primulina zixingensis Li H. Yang \& B. Pan (Gesneriaceae) Annales Botanici Fennici 57 (1-3): 55. 2020.

TYPE: CHINA (Hunan*), 10/2/2017, Li H. Yang YLH433 (holotype: IBSC); Bo Pan PB2018071701 (paratype: IBK).

Pseuderanthemum arunachalense D. Borah, R. Kr. Singh \& Taram (Acanthaceae) Indian Forester 146 (7): 660. 2020. 
杜诚, 刘军, 叶文, 廖帅, 葛斌杰, 刘冰, 马金双 (2021) 中国植物新分类群、新名称 2020 年度报告. 生物多样性, 29, 10111020. http://www.biodiversity-science.net/CN/10.17520/biods.2021122

TYPE: CHINA (Xizang*), 11/27/2016, D. Borah \& M. Taram 64 (holotype: ASSAM; isotype: ARUN).

Pseudocayratia orientalisinensis Z. H. Chen, W. Y. Xie \& X. F. Jin (Vitaceae) Phytotaxa 475 (4): 261. 2020.

TYPE: CHINA (Zhejiang*), 8/4/2017, Z. H. Chen, J. Lin \& R. L. Liu JN17080401 (holotype: ZM; isotype: HTC, PE); P. C. Tsoong 4014 (paratype: PE). M. J. Wang 0615 (paratype: LBG); C. M. Tan 97577 (paratype: JJF); G. X. Chen \& D. G. Zhang LXP-064986 (paratype: JIU); G. X. Chen \& D. G. Zhang LXP-06-4093 (paratype: JIU); Z. H. Chen \& al. JN17062501 (paratype: ZM); Z. H. Chen \& al. JN17062502 (paratype: ZM); Z. H. Chen, R. L. Liu \& X. D. Mei JN18051907 (paratype: ZM); Z. H. Chen, J. Lin \& J. S. Wang JN18062001 (paratype: HTC); Z. H. Chen, J. Lin \& J. S. Wang JN18062002 (paratype: HTC); X. D. Mei \& J. S. Wang JN18062320 (paratype: HTC); Z. H. Chen, J. F. Wang \& D. H. Wu LD18052002 (paratype: ZM); Z. H. Chen \& Y. Luo TMS-01 (paratype: ZM); H. Q. Zhu 000040 (paratype: HHBG, WUK); Y. Y. Ho 22309 (paratype: HHBG, PE); Y. Y. Ho 22319 (paratype: WUK); T. Tang \& W. Y. Hsia 437 (paratype: LBG); T. Tang \& W. Y. Hsia 241 (paratype: PE); T. Tang \& W. Y. Hsia 242 (paratype: PE); M. B. Deng \& al. 4297 (paratype: PE); T. N. Liu 138 (paratype: PE, WUK); H. Migo s.n. (paratype: PE); Z. H. Chen \& H. W. Zhang LA17061817 (paratype: ZM).

Pternopetalum paucifoliolatum J. F. Ye, X. Jie Li \& Ce H. Li (Apiaceae) PhytoKeys 166: 99. 2020.

TYPE: CHINA (Sichuan*), 5/2/2020, X. Jie Li CPG41074 (holotype: PE).

Quercus dehangensis G. X. Chen, D. G. Zhang \& B. Z. Wang (Fagaceae) Acta Botanica Boreali-Occidentalia Sinica 40 (10): 1781. 2020.

TYPE: CHINA (Hunan*), 11/7/2019, G. X. Chen, D. G. Zhang \& B. Z. Wang DHK-03-1001 (holotype: JIU; isotype: JIU).

Rhamnella intermedia Z. Qiang Lu \& Y. Shuai Sun (Rhamnaceae) PhytoKeys 159: 119. 2020.

TYPE: CHINA (Guangxi*), 10/5/2019, Z. Qiang Lu 2019YG2601 (holotype: GXMI; isotype: HITBC).

Rhododendron pudingense X. Y. Dai, C. H. Yang \& Y. P. Ma (Ericaceae) PhytoKeys 146: 54. 2020.

TYPE: CHINA (Guizhou*), 5/7/2018, X. Y. Dai 180507112 (holotype: GF; isotype: KUN, PE).

Rhododendron xishuiense C. H. Yang \& C. D. Yang (Ericaceae) Phytotaxa 429 (3): 248. 2020.

TYPE: CHINA (Guizhou*), 7/21/2010, C. H. Yang 5784 (holotype: GF-09035587); C. H. Yang 7590 (paratype: GF).

Rhynchotechum nirijuliense Taram \& D. Borah (Gesneriaceae) The Gardens' Bulletin Singapore 72 (1): 126. 2020.

TYPE: CHINA (Xizang*), 5/1/2019, M. Taram \& D. Borah 0157 (holotype: CAL; isotype: ASSAM, ARUN, E).

Rubus jingningensis Z. H. Chen, F. Chen \& F. G. Zhang (Rosaceae) Journal of Hangzhou Normal University (Natural Science Edition) 19 (3): 244. 2020.

TYPE: CHINA (Zhejiang*), 7/4/2019, F. Chen, Z. H. Chen \& X. D. Mei JN19070403 (holotype: ZM; isotype: ZM); J. Lin, Y. K. Xu \& C. G. Zhao JN19081906 (paratype: ZM); J. Lin, Y. K. Xu \& C. G. Zhao JN19081910 (paratype: ZM); Z. H. Chen, X. D. Mei \& H. F. Xu JN19081903 (paratype: ZM); Z. H. Chen, X. D. Mei \& H. F. Xu JN19081904 (paratype: ZM); J. Lin, Y. K. Xu \& C. G. Zhao JN19081908 (paratype: ZM); J. Lin, Y. K. Xu \& C. G. Zhao JN19081911 (paratype: ZM); F. Chen \& Z. H. Chen JN19073003 (paratype: ZM); X. D. Mei, C. G. Zhao \& H. F. Xu JN19081916 (paratype: ZM); Z. H. Chen \& H. F. Xu JN19090103 (paratype: ZM); X. D. Mei, D. H. Wu \& Z. H. Chen JN19081702 (paratype: ZM); X. D. Mei, D. H. Wu \& Z. H. Chen JN19081703 (paratype: ZM).

Salacia malipoensis X. D. Ma \& J. Y. Shen (Celastraceae) Annales Botanici Fennici 57 (1-3): 110. 2020.

TYPE: CHINA (Yunnan*), 4/8/2019, J. Y. Shen \& X. D. Ma 1488 (holotype: HITBC; isotype:HIB, HITBC, KUN, PE).

Saussurea sagittifolia Y. S. Chen \& S. R. Yi (Asteraceae) Phytotaxa 472 (3): 295. 2020.

TYPE: CHINA (Sichuan*), 9/5/2020, L. S. Xu XL200484 (holotype: IBSC; isotype: KUN, PE).

Saussurea solaris Raab-Straube \& Lidén (Asteraceae) Symbolae Botanicae Upsalienses, Acta Universitatis Upsaliensis 40: 130.2020. TYPE: CHINA (Xizang*), 9/9/1938, F. Kingdon-Ward 14229 (holotype: BM-000535025).

Saussurea xinjiangensis Y. S. Chen (Asteraceae) Nordic Journal of Botany 38 (e02711): 2. 2020.

TYPE: CHINA (Xinjiang*), 8/15/2014, Y. S. Chen \& Q. Yuan 141562 (holotype: PE-02053504; isotype: PE-02053337, PE-02053501, PE-02053502, PE-02053503, PE-02053505).

Saussurea yilingii Y. S. Chen (Asteraceae) Phytotaxa 452 (3): 236. 2020.

TYPE: CHINA (Sichuan*), 9/1/2015, FLPH Sichuan Expedition (Y. S. Chen \& al.) 151871 (holotype: PE; isotype: PE).

Saussurea yiwuensis L. Q. Zhao \& R. Xu (Asteraceae) Annales Botanici Fennici 57 (1-3): 159. 2020.

TYPE: CHINA (Xinjiang*), 7/30/2016, L. Q. Zhao, R. Xu \& Z. Y. Yao XJ16-7001 (holotype: HIMC; isotype: HIMC).

Sedum nanlingense Yan Liu \& C. Y. Zou (Crassulaceae) Phytotaxa 447 (3): 183.2020.

TYPE: CHINA (Guangxi*), 8/12/2016, s.coll. 450329160812022LY (holotype: IBK); s.coll. 450329161028010LY (paratype: IBK).

Selaginella subvaginata X. C. Zhang \& Shalimov (Selaginellaceae) Journal of Species Research 9 (3): 222. 2020. 
杜诚, 刘军, 叶文, 廖帅, 葛斌杰, 刘冰, 马金双 (2021) 中国植物新分类群、新名称 2020 年度报告. 生物多样性, 29, 10111020. http://www.biodiversity-science.net/CN/10.17520/biods.2021122

TYPE: CHINA (Sichuan*), 8/18/2017, X. C. Zhang, R. H. Jiang \& E. F. Huang 8838 (holotype: PE; isotype: PE).

Sinosasa fanjingshanensis N. H. Xia, Q. M. Qin \& J. B. Ni (Poaceae) Taxon 70 (1): 34. 2020.

TYPE: CHINA (Guizhou), 9/6/2017, X. R. Zheng \& J. B. Ni 29 (holotype: IBSC-0857169).

Sinosasa huapingensis N. H. Xia, Q. M. Qin \& Y. H. Tong (Poaceae) Taxon 70 (1): 39. 2020.

TYPE: CHINA (Guangxi), 10/29/2015, Y. H. Tong \& N. H. Xia 517 (holotype: IBSC-0857168).

Sinosasa mingyueshanensis N. H. Xia, Q. M. Qin \& X. R. Zheng (Poaceae) Taxon 70 (1): 42.2020.

TYPE: CHINA (Jiangxi), 8/25/2017, X. R. Zheng 16 (holotype: IBSC-0857171).

Sinosasa polytricha N. H. Xia, Q. M. Qin \& X. R. Zheng (Poaceae) Taxon 70 (1): 43. 2020.

TYPE: CHINA (Guangdong, Hunan), 10/3/2017, X. R. Zheng \& N. H. Xia 130 (holotype: IBSC-0857170); L. Deng 5827 (paratype: IBSC); Z. P. Wang \& S. T. Liu 780048 (paratype: N); N. H. Xia \& J. B. Ni 14 (paratype: IBSC); C. X. Zeng \& Y. X. Zhang 06123 (paratype: KUN); L. Deng 291 (paratype: IBSC); S. H. Chen 2812 (paratype: IBSC).

Sinosenecio ovatifolius C. Y. Zou, Yan Liu \& Ying Liu (Asteraceae) Phytotaxa 460 (2): 155. 2020.

TYPE: CHINA (Guangxi*), 3/21/2020, C. Y. Zou, W. B. Xu \& J. Q. Huang ZCY 266 (holotype: PE; isotype: A).

Sinoseris changii Ze H. Wang, N. Kilian \& H. Peng (Asteraceae) Willdenowia 50 (1): 108. 2020.

TYPE: CHINA (Yunnan(T)), 10/12/2017, Ze H. Wang \& Y. P. Chen WZH20171001 (holotype: KUN; isotype: B, KUN, PE).

Sonneratia $\times$ zhongcairongii Y. S. Wang \& S. H. Shi (Lythraceae) PhytoKeys 154: 3. 2020.

TYPE: CHINA (Hainan*), 6/13/2018, C. R. Zhong Saa20180613-001 (holotype: IBSC; isotype: IBSC).

Sorbus gongshanensis X. F. Gao \& Meng Li (Rosaceae) PhytoKeys 144: 2. 2020.

TYPE: CHINA (Xizang, Yunnan*), 9/1/2006, Gaoligong Shan Biodiversity Survey (2006) 31749 (holotype: GH; isotype: CAS, KUN).

Spathoglottis arunachalensis Tsering \& K. Prasad (Orchidaceae) Phytotaxa 432 (3): 289. 2020.

TYPE: CHINA (Xizang*), 10/4/2016, J. Tsering 40642 (holotype: OHT; isotype: CAL, OHT).

Stellaria multipartita Bo Xu bis \& Meng Li (Caryophyllaceae) Phytotaxa 442 (3): 198. 2020.

TYPE: CHINA (Chongqing*), 8/4/2019, Meng Li 00785 (holotype: NF-2007616; isotype: CDBI, NF-2007617); G. H. Yang 58565 (paratype: PE-00612811).

Stellaria pentastyla W. Q. Wang, Han F. Xu \& Z. H. Ma (Caryophyllaceae) Phytotaxa 435 (1): 71. 2020.

TYPE: CHINA (Yunnan*), 6/8/2017, Xu \& Mou YN0014 (holotype: GAUA; isotyepe: IBSC).

Stellaria procumbens Huan C. Wang \& Feng Yang (Caryophyllaceae) Phytotaxa 435 (2): 195. 2020.

TYPE: CHINA (Sichuan, Yunnan*), 7/12/2019, Huan C. Wang \& al. LQ 7217 (holotype: YUKU; isotyepe: YUKU); Z. T. Guan 7620 (paratype: PE); Sichuan Vegetation Team 14096 (paratype: CDBI); F. T. Wang 23373 (paratype: KUN, PE, WUK); F. T. Wang 23438 (paratype: KUN, PE); F. T. Wang 75095 (paratype: WUK); Nanshuibeidiao Team 01879 (paratype: PE); Botanical Team of Sichuan Agricultural College 00997 (paratype: CDBI); W. B. Ju \& al. AZH01164 (paratype: CDBI); S. G. Wu 8138 (paratype: HITBC, KUN); C. W. Wang 63976 (paratype: PE, NAS, WUK); C. W. Wang 68491 (paratype: KUN, PE); C. W. Wang 64682 (paratype: PE); Sino-US Joint Plant Expedition 858 (paratype: KUN); C. Y. Wu \& al. 1454/2 (paratype: PE); H. C. Wang \& al. 0803 (paratype: YUKU); H. C. Wang \& al. 0497 (paratype: YUKU); H. C. Wang \& al. LQ 3196 (paratype: YUKU).

Stereochilus arunachalensis Chowlu \& A. N. Rao (Orchidaceae) Phytotaxa 433 (2): 177. 2020.

TYPE: CHINA (Xizang*), 6/4/2018, Chowlu 41000 (holotype: ARUN).

Stixis villiflora J. Y. Shen, S. Landrein, W. G. Wang \& X. D. Ma (Resedaceae) Taiwania 65 (1): 10. 2020.

TYPE: CHINA (Yunnan*), 3/16/2019, J. Y. Shen, X. D. Ma \& W. G. Wang 1413 (holotype: HITBC; isotype: HITBC, HIB, KUN).

Strobilanthes sunhangii T. Deng, J. T. Chen \& Y. F. Deng (Acanthaceae) PhytoKeys 166: 119. 2020.

TYPE: CHINA (Xizang*), 10/5/2018, H. Sun, T. Deng \& Z. M. Li Sunhang19964 (holotype: KUN-1345286; isotype: IBSC, KUN1345287, KUN-1345288).

Synotis panzhouensis M. Tang \& H. L. Zheng (Asteraceae) PhytoKeys 166: 80. 2020.

TYPE: CHINA (Guizhou*), 9/1/2020, Z. Li \& M. Tang 2020-0901 (holotype: JXAU; isotype: GZAC, JXAU).

Taraxacum dilutissimum Kirschner \& Štěpánek (Asteraceae) Phytotaxa 457 (1): 227. 2020.

TYPE: CHINA (Xizang), INDIA*, UZBEKISTAN, 4/20/2004, L. Klimeš 6629 (holotype: PRA; isotype: PRA). 
杜诚, 刘军, 叶文, 廖帅, 葛斌杰, 刘冰, 马金双 (2021) 中国植物新分类群、新名称 2020 年度报告. 生物多样性, 29, 10111020. http://www.biodiversity-science.net/CN/10.17520/biods.2021122

Taraxacum hedinii G. Haglund ex Kirschner \& Štěpánek (Asteraceae) Phytotaxa 457 (1): 185. 2020.

TYPE: CHINA (Xinjiang), INDIA*, 8/23/2002, L. Klimeš 5198 (holotype: PRA; isotype: PRA).

Taraxacum tubulosum Kirschner \& Štěpánek (Asteraceae) Phytotaxa 457 (1): 63.2020.

TYPE: CHINA (Xinjiang), INDIA*, 9/16/2002, L. Klimeš 3762 (holotype: PRA; isotype: S, BM, M).

Thalictrum jiulongense W. T. Wang (Ranunculaceae) Bulletin of Botanical Research 40 (1): 9. 2020.

TYPE: CHINA (Sichuan*), 7/17/2018, S. R. Zhang, J. Yang \& W. Zhang GGS10362 (holotype: PE-02293703).

Thalictrum kangdingense W. T. Wang (Ranunculaceae) Bulletin of Botanical Research 40 (1): 5.2020.

TYPE: CHINA (Sichuan*), 7/15/2018, X. Y. Zhu \& al. GG226 (holotype: PE-02293701).

Thalictrum tenuicaule W. T. Wang (Ranunculaceae) Bulletin of Botanical Research 40 (1): 7. 2020.

TYPE: CHINA (Sichuan*), 7/20/2018, Y. Yang \& al. GGS181 (holotype: PE-02293702).

Thismia jianfenglingensis Han Xu, H. J. Yang \& S. Q. Fang (Burmanniaceae) Phytotaxa 429 (2): 180. 2020.

TYPE: CHINA (Hainan*), 6/20/2017, Han Xu 2017001 (holotype: CANT; isotype: IBSC).

Tongoloa arguta L. J. Gui \& X. J. He (Apiaceae) PhytoKeys 164: 14. 2020.

TYPE: CHINA (Sichuan*), 9/26/2019, L. J. Gui GLJ19092601 (holotype: SZ).

Tongoloa tagongensis L. J. Gui \& X. J. He (Apiaceae) Phytotaxa 461 (1): 17. 2020.

TYPE: CHINA (Sichuan*), 9/21/2018, L. J. Gui \& S. B. Jia GLJ18092101 (holotype: SZ); Y. J. Li 401 (paratype: CDBI).

Trapa dimorphocarpa Z. S. Diao (Lythraceae) Guihaia 40 (1): 9. 2020.

TYPE: CHINA (Fujian*), 6/1/1985, L. Su 1989 (lectotype: YZU); Z. S. Diao 1989 (syntype: YZU); Fujian Agri. Coll. 1911 (syntype: YZU).

Trichosanthes glandulosa G. Q. Zhu, H. Z. Peng \& X. H. Liu (Cucurbitaceae) Journal of Zhejiang Forestry Science and Technology 40 (3): 91. 2020.

TYPE: CHINA (Zhejiang*), 7/3/2018, G. Q. Zhu ZFAZGQ2018070301 (holotype: ZM); L. Hong HZ056319 (paratype: HHBG); G. Q. Zhu ZFAZGQ201308261 (paratype: ZM); G. Q. Zhu \& al. 2017102232 (paratype: ZM); G. Q. Zhu ZFAZGQ2016071701 (paratype: ZM); G. Q. Zhu \& al. ZFAZGQ2016092407 (paratype: ZM); S. Z. Yang 139 (paratype: ZM); G. Q. Zhu ZFAZGQ2018102601 (paratype: ZM).

Trigonotis motuoensis Q. R. Liu \& Xue M. Xu (Boraginaceae) Phytotaxa 461 (4): 237. 2020.

TYPE: CHINA (Xizang*), 5/28/2019, Хиe M. Xu \& D. H. Liu BNU2019XZ182 (holotype: BNU-0049249; isotype: BNU).

Tupistra nganii K. S. Nguyen, Aver., N. Tanaka \& Nuraliev (Asparagaceae) Phytotaxa 449 (2): 174. 2020.

TYPE: CHINA (Yunnan), VIETNAM*, 10/19/2018, L. V. Averyanov, N. S. Khang, T.V. Maisak, T. Q. Ngan, VR 1015 (holotype: HN; isotype: HN, LE-01042176, LE-01042177, LE-01042186); W. J. Kress, L. M. Prince \& Y. M. Xia 00-6803 (paratype: US-00732584); N. Q. Hieu \& al. CPC 4976 (paratype: LE-01070256); L. V. Averyanov, N. S. Khang \& T. V. Maisak VR 1452 (paratype: HN; LE01061076, LE-01066967); N. S. Khang \& al. NSK 1182 (paratype: HN; LE-01070257); M. S. Nuraliev, Yu. O. Kopylov-Guskov, D. F. Lyskov 3025 (paratype: MW-0595720, MW-0595721; LE-01070259).

Vaccinium napoense Y. H. Tong \& N. H. Xia (Ericaceae) Nordic Journal of Botany 38 (e02773): 2. 2020.

TYPE: CHINA (Guangxi*), 3/29/2019, Y. H. Tong \& Z. Y. Cai TYH-2093 (holotype: IBSC; isotype: IBK); Y. S. Huang \& al. Y1077 (paratype: IBK).

Viburnum fengyangshanense Z. H. Chen, P. L. Chiu \& L. X. Ye (Adoxaceae) Journal of Hangzhou Normal University (Natural Science Edition) 19 (3): 261. 2020.

TYPE: CHINA (Zhejiang*), 5/8/2016, Z. H. Chen, L. X. Ye \& S. L. Liu LQ2016001 (holotype: ZM; isotype: HTC,ZM); S. Y. Zhang 3182 (paratype: PE, IBSC); Z. H. Chen, L. X. Ye \& S. L. Liu LQ2016003 (paratype: ZM); L. X. Ye \& S. L. Liu LQ2016005 (paratype: ZM); Z. H. Chen, L. X. Ye \& S. L. Liu LQ2016006 (paratype: ZM); Z. H. Chen, S. L. Liu \& J. S. Wang LQ2018001 (paratype: ZM); X. F. Jin 4210 (paratype: HTC); X. F. Jin 4211 (paratype: HTC); X. F. Jin 4212 (paratype: HTC); X. F. Jin 4216 (paratype: HTC); Z. H. Chen \& al. LQFYS180720-09 (paratype: ZM); Z. H. Chen \& al. LQFYS180721-03 (paratype: ZM); Z. H. Chen \& al. LQFYS18072104 (paratype: ZM); Z. H. Chen \& al. 8 (paratype: ZM).

Zingiber chengii Y. H. Tseng, C. M. Wang \& Yuan C. Lin (Zingiberaceae) PhytoKeys 139: 3. 2020.

TYPE: CHINA (Taiwan*), 5/23/2014, Y. H. Tseng 5614 (holotype: TCF).

Zingiber porphyrochilum Y. H. Tan \& H. B. Ding (Zingiberaceae) Annales Botanici Fennici 57 (4-6): 197. 2020.

TYPE: CHINA (Yunnan*), 8/17/2018, Y. H. Tan, B. Yang, H. B. Ding \& X. D. Zeng Y0143 (holotype: HITBC). 